\title{
ER-12-1 COMPLETION REPORT
}

\author{
Prepared by \\ Charles E. Russell, David Gillespie \\ James C. Cole \\ S.L. Drellack, L.B. Prothro, P.H. Thompson, R.L. McCall \\ Gayle A. Pawloski, and Richard Carlson
}

Submitted to

Nevada Operations Office

U.S. Department of Energy
RECEIVED

APR 221997

OSTI

December 1996

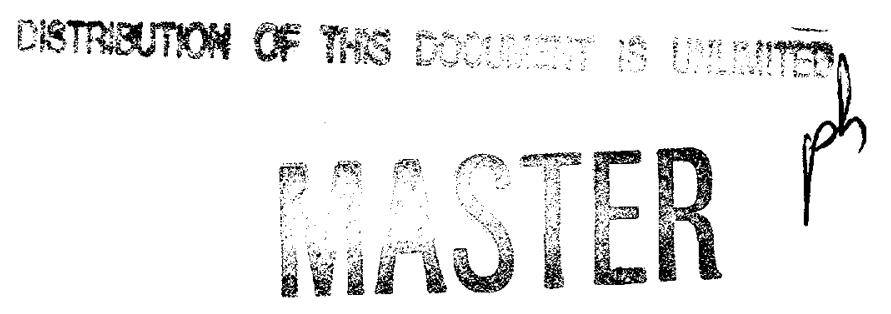



This report was prepared as an account of work sponsored by the United States Government. Neither the United States nor the United States Department of Energy, nor any of their employees, makes any warranty, express or implied, or assumes any legal liability or responsibility for the accuracy, completeness or usefulness of any information, apparatus, product or process disclosed, or represents that its use would not infringe privately owned rights. Reference herein to any specific commercial product, process, or service by trade name, mark, manufacturer, or otherwise, does not necessarily constitute or imply its endorsement, recommendation, or favoring by the United States Government or any agency thereof. The views and opinions of authors expressed herein do not necessarily state or reflect those of the United States Government or any agency thereof.

This report has been reproduced directly from the best available copy.

Available to DOE and DOE contractors from the Office of Scientific and Technical Information, P.O. Box 62, Oak Ridge, TN 37831; prices available from (423) 576-8401.

Available to the public from the National Technical Information Service, U.S. Department of Commerce, 5285 Port Royal Rd., Springfield, VA 22161. 



\section{DISCLAIMER}

Portions of this document may be illegible in electronic image products. Images are produced from the best available original document. 



\title{
ER-12-1 COMPLETION REPORT
}

\author{
by \\ Charles E. Russell, David Gillespie ${ }^{1}$ \\ James C. Cole ${ }^{2}$ \\ S.L. Drellack, L.B. Prothro, P.H. Thompson, R.L. McCall ${ }^{3}$ \\ Gayle A. Pawloski and Richard Carlson ${ }^{4}$
}

Publication No. 45120

prepared for

Nevada Operations Office

U.S. Department of Energy

Las Vegas, Nevada

December 1996

\footnotetext{
${ }^{1}$ Desert Research Institute

${ }^{2}$ U.S. Geological Survey

${ }^{3}$ Raytheon Services Nevada

${ }^{4}$ Lawrence Livermore National Laboratory
}

The work upon which this report is based was supported by the U.S. Department of Energy under Contract \#DE-AC08-90NV10845. 



\section{CONTENTS}

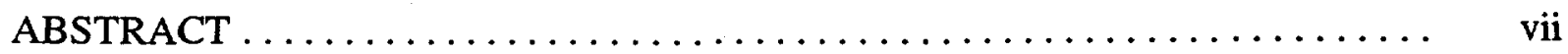

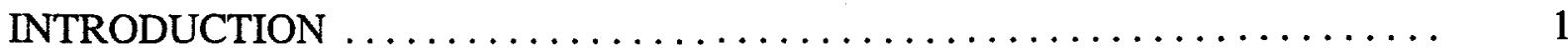

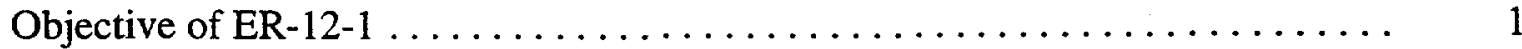

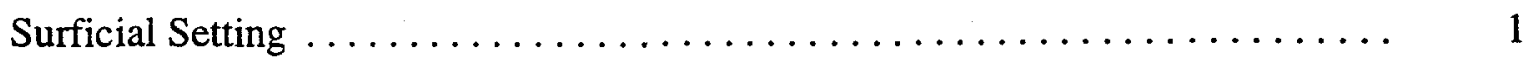

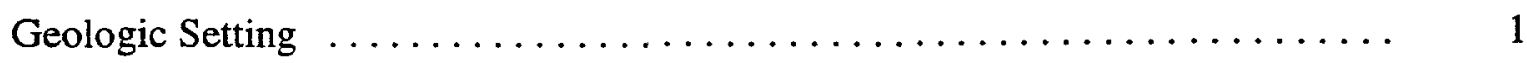

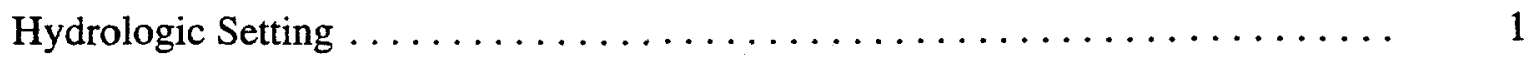

Location with Respect to Testing $\ldots \ldots \ldots \ldots \ldots \ldots \ldots \ldots \ldots \ldots \ldots \ldots \ldots \ldots$

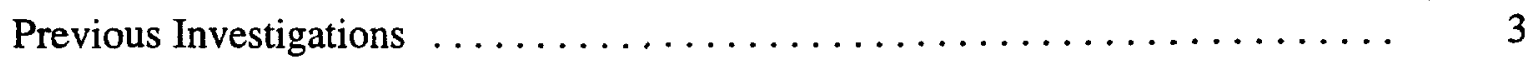

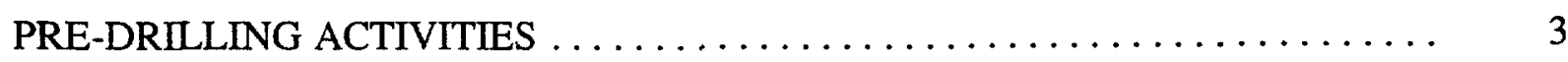

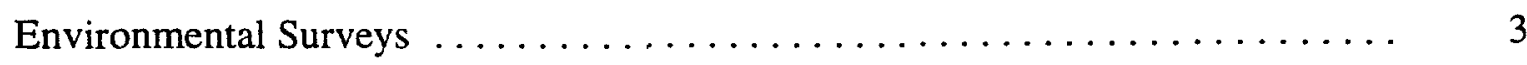

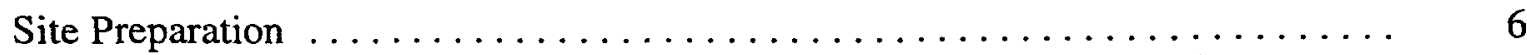

DRILLING TECHNIQUES AND HOLE HISTORY . . . . . . . . . . . . 6

GEOLOGY OF ER-12-1 BASED ON MACROSCOPIC OBSERVATIONS $\ldots \ldots .22$

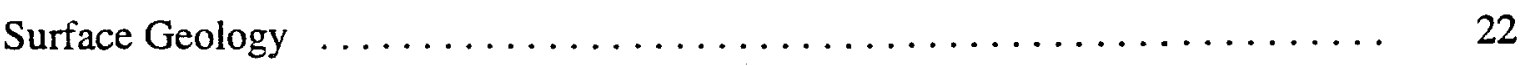

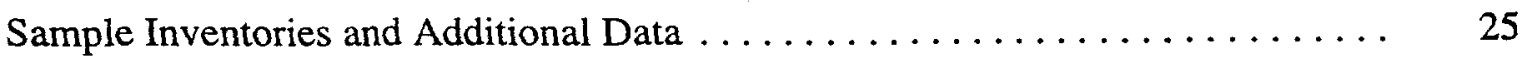

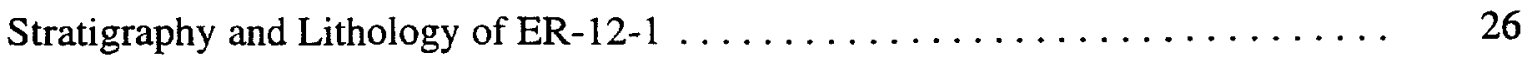

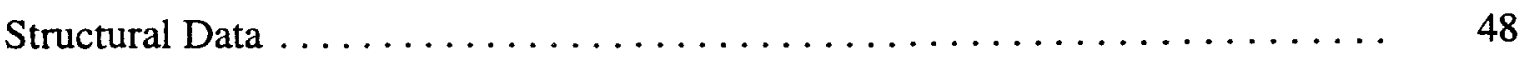

STRATIGRAPHY, STRUCTURE AND THERMAL HISTORY OF ROCKS

PENETRATED BY WELL ER-12-1 BASED UPON MICROSCOPIC

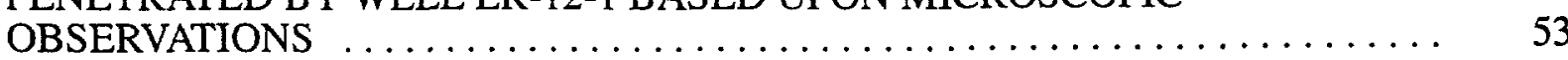

Lithology, Stratigraphy, and Paleontology ................... 57

Thermal History of the Rocks at the ER-12-1 Well . . . . . . . . . . . . 66

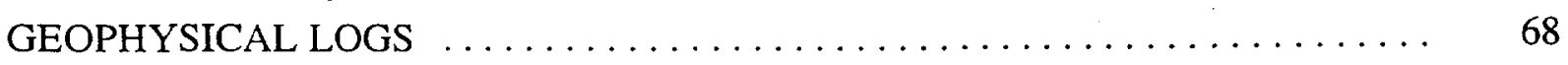

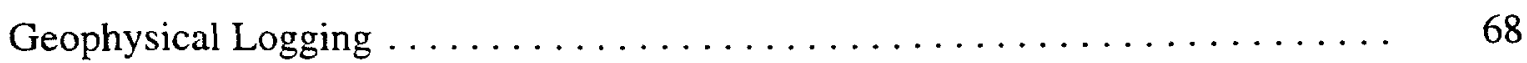

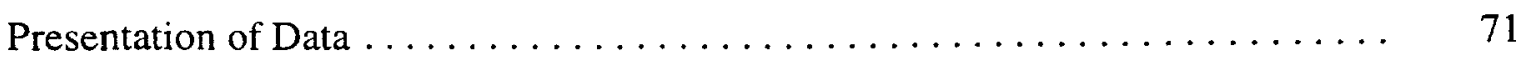

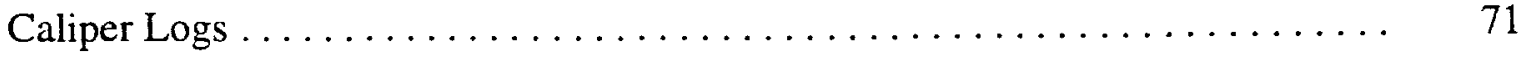

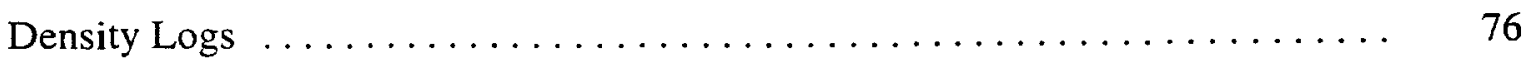

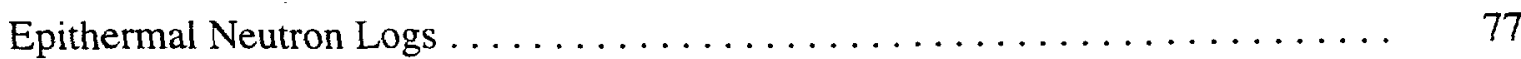

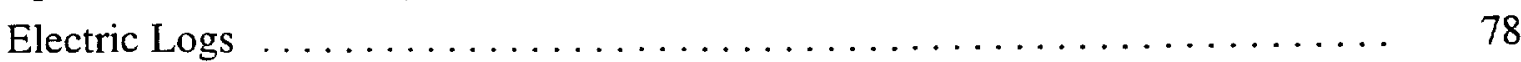

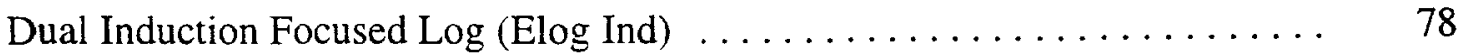

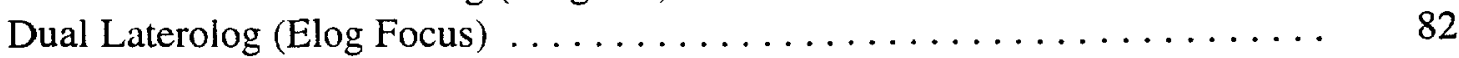

Microlog (Elog Mini) . . . . . . . . . . . . . . . . . . . . . . . 82

Formation Micro-Scanner Log (Dip Micro) $\ldots \ldots \ldots \ldots \ldots \ldots \ldots \ldots, \quad 82$

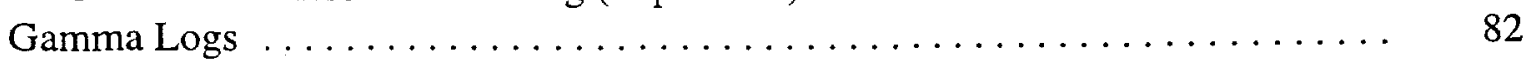

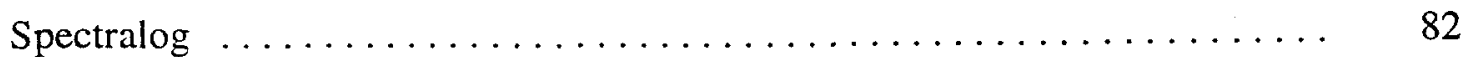

Gamma ....................................... ${ }_{85}$

Total Intensity Magnetometer $\operatorname{Logs} \ldots \ldots \ldots \ldots \ldots \ldots \ldots \ldots \ldots \ldots \ldots$ 


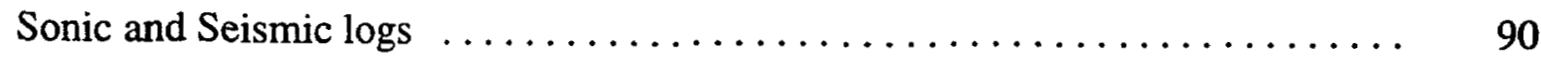

Presentation of Data from Specific Intervals . . . . . . . . . . . . . . 91

Screened Intervals $\ldots \ldots \ldots \ldots \ldots \ldots \ldots \ldots \ldots \ldots \ldots \ldots, \quad 91$

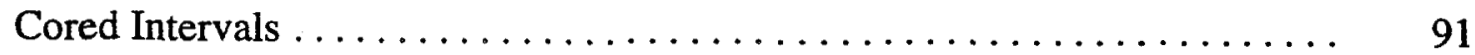

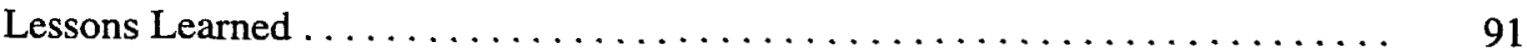

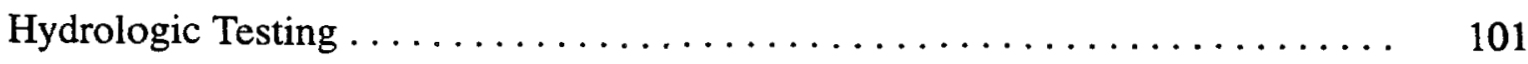

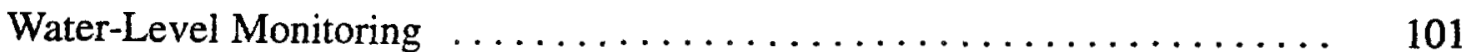

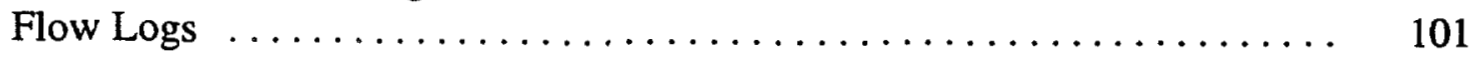

Multiple Rate Test $\ldots \ldots \ldots \ldots \ldots \ldots \ldots \ldots \ldots \ldots \ldots \ldots \ldots \ldots, 105$

Radially Converging Aquifer Test $\ldots \ldots \ldots \ldots \ldots \ldots \ldots \ldots \ldots, 110$

Drill-Stem Tests ................................ 113

Radially Converging Aquifer Test of the Upper Zone ............. 122

Hydrochemistry .................................. 124

Field Measurements During Drilling $\ldots \ldots \ldots \ldots \ldots \ldots \ldots \ldots \ldots \ldots . \quad 125$

Field Measurements During Well Development and Testing $\ldots \ldots \ldots \ldots .125$

Field Measurement Quality Control/Quality Assurance ............. 131

Geochemical Water-Quality Samples $\ldots \ldots \ldots \ldots \ldots \ldots \ldots \ldots \ldots . . \ldots \ldots$

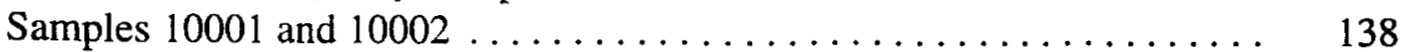

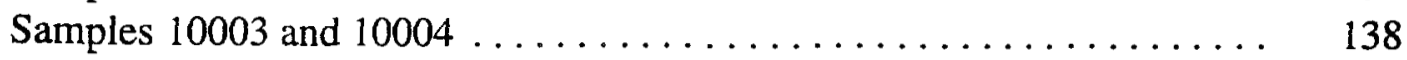

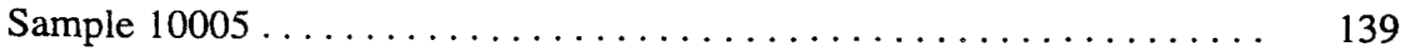

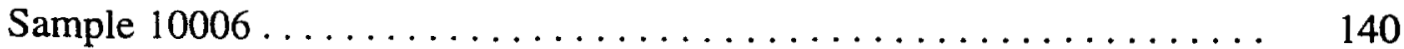

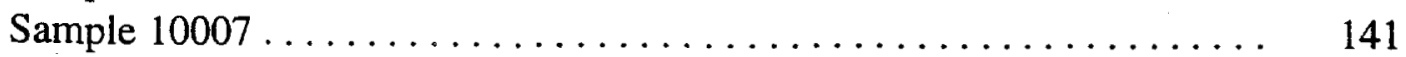

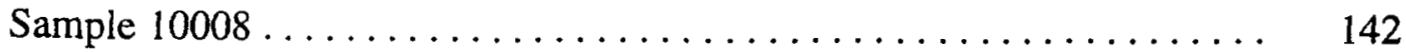

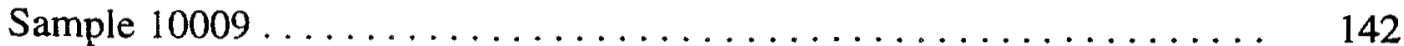

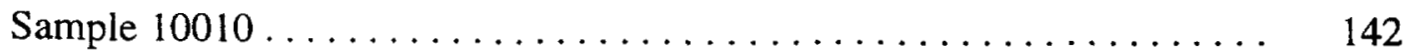

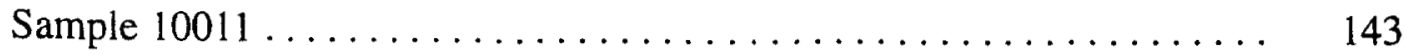

Geochemical Characterization Sample ..................... 143

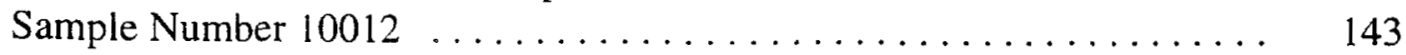

SUMMARY

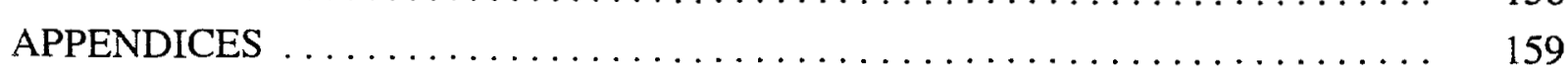

Appendix A. Geologic Prediction Report ...................... 159

Appendix B. Hydrologic Prediction Report ................... 171

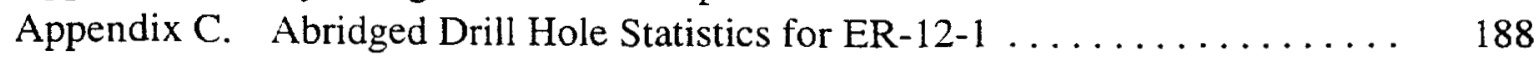

Appendix D. Sequence of Events at ER-12-1 ................ 191

\section{FIGURES}

1. Location of ER-12-1 ............................... 2

2. Schematic of ER-12-1 hole construction. ..................... 7

3. Geologic map of the ER-12-1 vicinity. $\ldots \ldots \ldots \ldots \ldots \ldots \ldots \ldots \ldots \ldots \ldots \ldots \ldots \ldots$ 


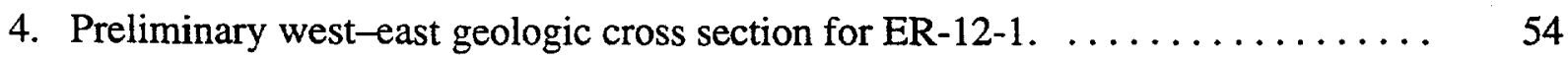

5. Summary of selected logs from the dry-hole logging suite. $\ldots \ldots \ldots \ldots \ldots$

6. Summary of selected logs from the wet-hole logging suite. $\ldots \ldots \ldots \ldots$

7. ER-12-1 caliper logs, 400 to $1100 \mathrm{~m}(1312$ to $3608 \mathrm{ft}) . \ldots \ldots \ldots \ldots \ldots \ldots$

8. Density, epithermal neutron, and sonic logs from ER-12-1, 0 to $600 \mathrm{~m}$ (0 to $1968 \mathrm{ft})$.

9. Density epithermal neutron, continuous velocity and sonic logs, 500 to $1100 \mathrm{~m}$

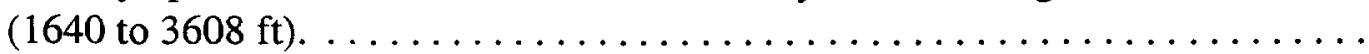

10. Deep, medium and focused resistivity, 0 to $600 \mathrm{~m}(0$ to $1968 \mathrm{ft}) \ldots \ldots \ldots \ldots$

11. Shallow and deep resistivity, 500 to $1100 \mathrm{~m}(1640$ to $3608 \mathrm{ft})$. . . . . . . 83

12. Self potential, 500 to $1100 \mathrm{~m}(1640$ to $3608 \mathrm{ft}) \ldots \ldots \ldots \ldots \ldots \ldots \ldots$

13. Spectral gamma logs, 0 to $600 \mathrm{~m}(0$ to $1968 \mathrm{ft}) \ldots \ldots \ldots \ldots \ldots \ldots \ldots$

14. Spectral gamma logs, 400 to $1000 \mathrm{~m}(1312$ to $3280 \mathrm{ft}) \ldots \ldots \ldots \ldots \ldots \ldots$

15. Borehole magnetometer logs, 0 to $600 \mathrm{~m}(0$ to $1968 \mathrm{ft}) \ldots \ldots \ldots \ldots \ldots$

16. Borehole magnetometer logs, 500 to $1100 \mathrm{~m}(1640$ to $3608 \mathrm{ft}) \ldots \ldots \ldots \ldots$

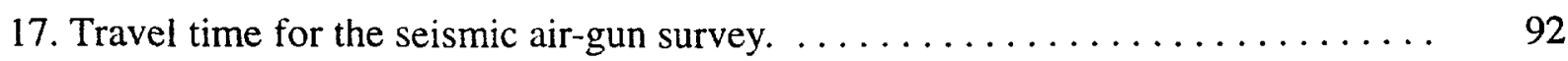

18. Selected logs for the screened interval of 518 to $554 \mathrm{~m}(1700$ to $1820 \mathrm{ft}) \ldots \ldots 93$

19. Selected $\log s$ for the screened interval of 585 to $597 \mathrm{~m}(1920$ to $1960 \mathrm{ft}) \ldots \ldots .994$

20. Selected logs for the screened interval of 765 to $789 \mathrm{~m} \mathrm{(2510} \mathrm{to} 2590 \mathrm{ft}) \ldots \ldots .95$

21. Selected logs for the screened interval of 914 to $963 \mathrm{~m} \mathrm{(3000} \mathrm{to} 3160 \mathrm{ft}$ ). . . . 96

22. Selected logs for the screened interval of 1024 to $1048 \mathrm{~m}$ (3360 to $3440 \mathrm{ft}$ ). . . $\quad 97$

23. Selected logs for the cored interval of 539 to $543 \mathrm{~m}(1769$ to $1784 \mathrm{ft})$ and 548 to $556 \mathrm{~m}(1798$ to $1825 \mathrm{ft}) \ldots \ldots \ldots \ldots \ldots \ldots \ldots \ldots \ldots \ldots \ldots \ldots$

24. Selected logs for the cored interval of 751 to $757 \mathrm{~m}(2464$ to $2486 \mathrm{ft})$. . . . . . 99

25. Selected $\operatorname{logs}$ for the cored interval of 895 to $903 \mathrm{~m}(2937$ to $2963 \mathrm{ft})$. . . . . 100

26. Results of the oxygen activation flow $\log . \ldots \ldots \ldots \ldots \ldots \ldots \ldots \ldots$

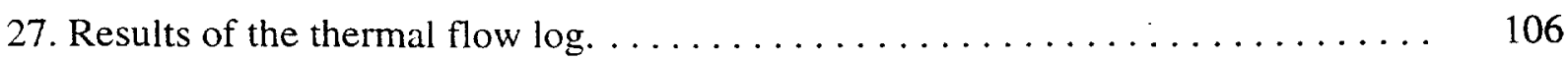

28. Downhole pressure response during multiple-rate and long-term aquifer test. . . $\quad 108$ 
29. Barometric pressure changes during multiple-rate and the long-term aquifer test. . . . . . . . . . . . . . . . . . . . . . . . . . . . . .

30. Cooper-Jacob analysis of drawdown data from long-term aquifer test at

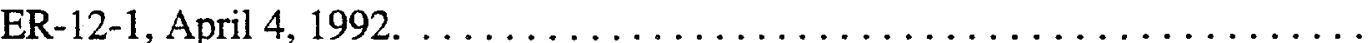

31. Hydrologic parameter estimation of ER-12-1 aquifer test, April 4, 1992. ..... 113

32. Pressure and temperature results of drill-stem test conducted on 915 to $963 \mathrm{~m}$ (3000 to $3160 \mathrm{ft}$ ) screened interval. . . . . . . . . . . . . . . . . . .

33. Pressure and temperature results of drill-stem test conducted on 765 to $790 \mathrm{~m}$ (2510 to $2550 \mathrm{ft}$ ) screened interval.

34. Pressure and temperature results of drill-stem test conducted on 585 to $597 \mathrm{~m}$ $(1920$ to $1960 \mathrm{ft})$ screened interval.

35. Results of drill-stem test conducted on the screened interval extending from $915 \mathrm{~m}$ to $963 \mathrm{~m}$ (3000 to $3160 \mathrm{ft}$ ) using an $\propto=10^{-8} \ldots \ldots \ldots \ldots \ldots \ldots \ldots$

36. Results of drill-stem test conducted on the screened interval extending from $915 \mathrm{~m}$ to $963 \mathrm{~m}$ (3000 to $3160 \mathrm{ft}$ ) using an $\propto=10^{-5} \ldots \ldots \ldots \ldots \ldots \ldots$

37. Results of drill-stem test conducted on the screened interval extending from 765 to $790 \mathrm{~m}$ ( 2510 to $2590 \mathrm{ft}$ ) using an $\propto=10^{-4} \ldots \ldots \ldots \ldots \ldots \ldots \ldots \ldots$

38. Reduction of ER-12-1 upper zone aquifer test, March 17, 1993. ........ 123

39. Hydrologic parameter estimation of ER-12-1, upper zone aquifer test, March 17, 1993.

40. Results of tritium analyses during drilling. $\ldots \ldots \ldots \ldots \ldots \ldots \ldots \ldots \ldots$

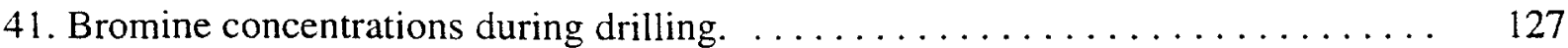

42. Tritium analyses during well development and testing. $\ldots \ldots \ldots \ldots \ldots \ldots \ldots, 128$

43. Bromine concentrations during well development and testing. ........... 130

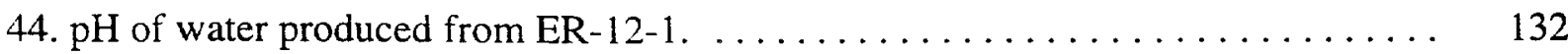

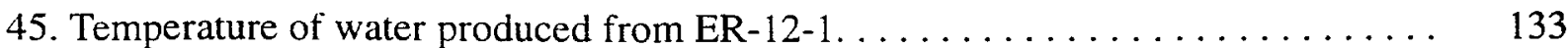

46. Turbidity of water produced from ER-12-1 . . . . . . . . . . . . . . . 134

47. Electrical conductivity of water produced from ER-12-1. . . . . . . . . 135

48. Stiff diagrams for carbonate, Eleana, and volcanic wells. . . . . . . . . 151

49. Stable isotopic signature of sample 10012 verses samples from Rainier Mesa seeps, carbonate wells and volcanic wells. 


\section{TABLES}

1. Drill Holes and Tunnels within $2590.8 \mathrm{~m}(8500 \mathrm{ft})$ Radius of ER-12-1. . . . . 4

2. Drilling Techniques used at ER-12-1. ................. 8

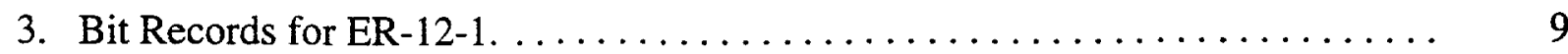

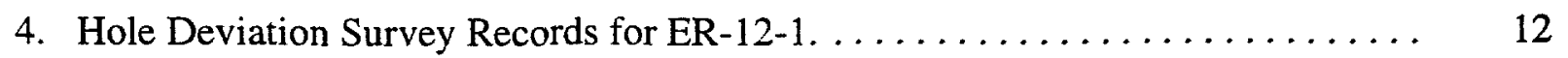

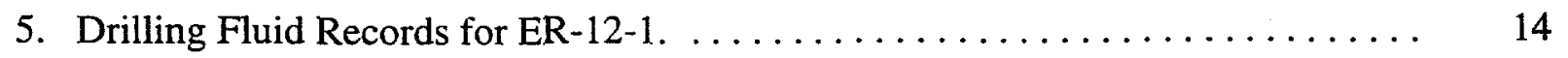

6. Drilling Mud Data for ER-12-1. $\ldots \ldots \ldots \ldots \ldots \ldots \ldots \ldots \ldots \ldots \ldots \ldots$

7. Drillers Notes. $\ldots \ldots \ldots \ldots \ldots \ldots \ldots \ldots \ldots \ldots \ldots \ldots \ldots \ldots \ldots \ldots$

8. Completion and Well Development Fluids and Materials Injected into ER-12-1. . 19

9. Rationale for Completion of Intervals in Well ER-12-1 . . . . . . . . . 20

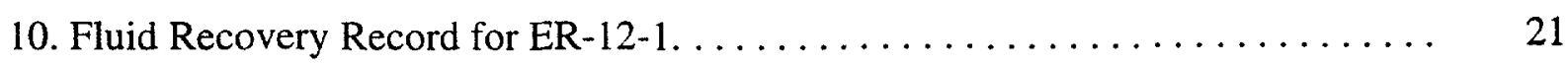

11. Correlation of Stratigraphic Nomenclature used in this Report with that used

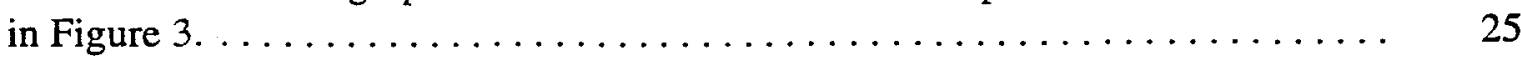

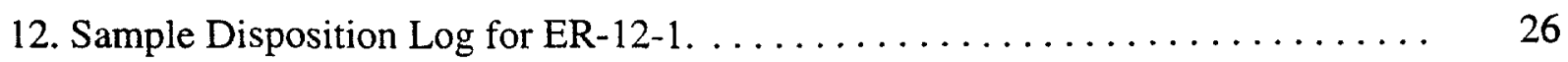

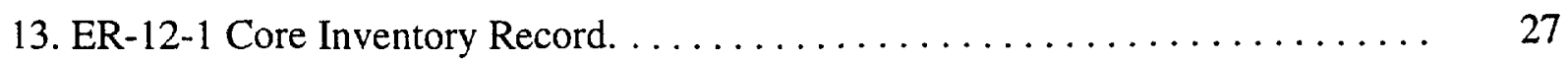

14. List of Polished Thin Sections Available from Selected ER-12-1

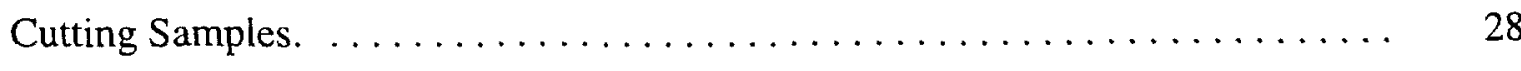

15. X-ray Diffraction Analyses for Selected ER-12-1 Cuttings Samples. . . . . . . . 29

16. Physical Properties Measurements on Selected ER-12-1 Samples. . . . . . . . 30

17. Downhole Camera Video Log for ER-12-1 (Surface to $486.3 \mathrm{~m}(0$ to $1596 \mathrm{ft}$ ))

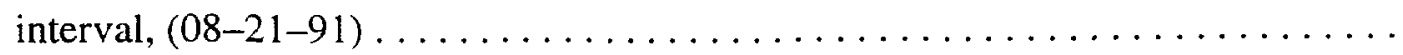

18. List of 35-mm Slides Documenting Drilling Activities, Equipment and

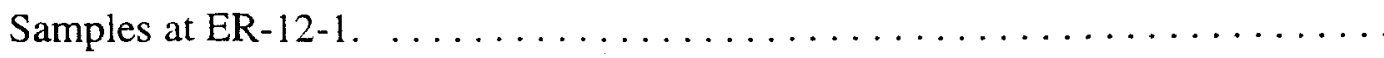

19. Stratigraphic Nomenclature used in this Report for the Pre-Tertiary Rocks at the NTS. . . . . . . . . . . . . . . . . . . . . . . .

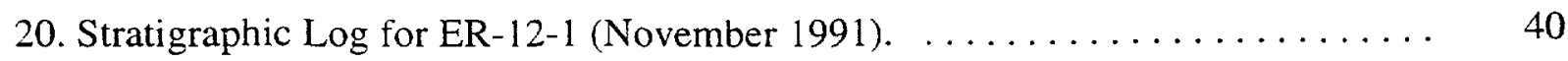

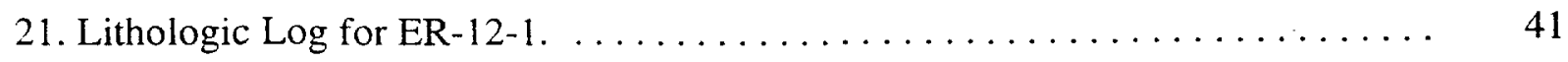

22. Description of Core \#1 $(539.2$ to $543.8 \mathrm{~m}) . \ldots \ldots \ldots \ldots \ldots \ldots \ldots \ldots$ 
23. Description of Core \#3 (751.0 to $757.7 \mathrm{~m}) \ldots \ldots \ldots \ldots \ldots \ldots \ldots \ldots \ldots \ldots$

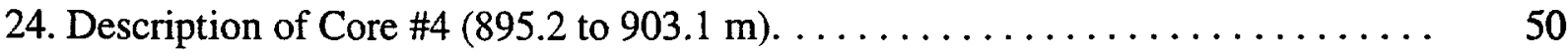

25. Selected Holes that Penetrate the Pre-Tertiary Rocks Surrounding ER-12-1. . . . 52

26. Rainier Mesa Drill Holes Penetrating Quartz Monzonite (Gold Meadows Stock). 53

27. Probable Faults Intersected in the ER-12-1 Borehole. ............. 55

28. Possible Faults and/or Fractured Intervals Intersected in the ER-12-1 Borehole. . 56

29. Stratigraphic Log for Groundwater Characterization Project Well ER-12-1 based on Paleontologic and Petrographic Examinations of Cuttings Samples Collected at $15.4-\mathrm{m}(50 \mathrm{ft})$ Intervals. . . . . . . . . . . . . . . . . 58

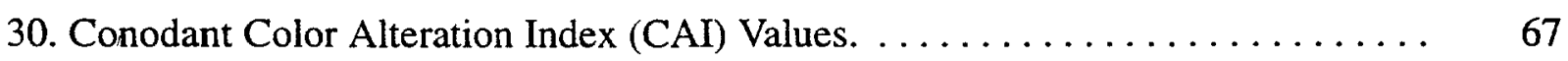

31. List of Geophysical Logs Acquired in ER-12-1 ................ 69

32. Processed Data from ER-12-1 Archived at LLNL. ................ 74

33. Depth to Fluid Level and Total Depth Measurements for ER-12-1, ....... 101

34. Static Pressures and Heads of Screened Intervals in Well ER-12-1 . . . . . . 102

35. Log Depth and Water Velocities from the Oxygen Activation Log. ........ 103

36. Ambient Flow Versus Depth, as Determined by the Thermal Flow Log. ...... 105

37. Maximum, Minimum, and Duration of Stress during Drill-stem Tests Conducted at ER-12-1......................................... 118

38. Geochemical Samples Acquired from Well ER-12-1 ............ 136

39. Geochemical Results from the Upper Zone of Well ER-12-1(518 to $555 \mathrm{~m}$ or

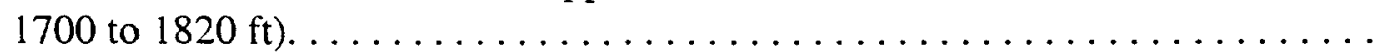

40. Stable Isotopic Signature of Wells and Seeps on the NTS. . . . . . . . . 155 


\section{ABSTRACT}

The objective of drillhole ER-12-1 was to determine the hydrogeology of paleozoic carbonate rocks and of the Eleana Formation, a regional aquitard, in an area potentially downgradient from underground nuclear testing conducted in nearby Rainier Mesa. This objective was addressed through the drilling of well ER-12-1 at N886,640.26 E640,538.85 Nevada Central Coordinates. Drilling of the $1094 \mathrm{~m}$ ( $3588 \mathrm{ft}$ ) well began on July 19, 1991 and was completed on October 17, 1991. Drilling problems included hole deviation and hole instability that prevented the timely completion of this borehole. Drilling methods used include rotary tri-cone and rotary hammer drilling with conventional and reverse circulation using air/water, air/foam (Davis mix), and bentonite mud.

Geologic cuttings and geophysical logs were obtained from the well. The rocks penetrated by the ER-12-1 drillhole are a complex assemblage of Silurian, Devonian, and Mississippian sedimentary rocks that are bounded by numerous faults that show substantial stratigraphic offset.

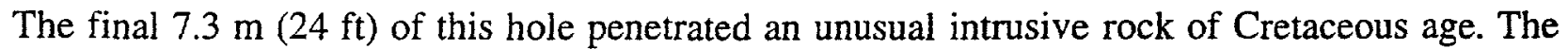
geology of this borehole was substantially different from that expected, with the Tongue Wash Fault encountered at a much shallower depth, paleozoic rocks shuffled out of stratigraphic sequence, and the presence of an altered biotite-rich microporphyritic igneous rock at the bottom of the borehole.

Conodont CAI analyses and rock pyrolysis analyses indicate that the carbonate rocks in ER-12-1, as well as the intervening sheets of Eleana siltstone, have been thermally overprinted following movement on the faults that separate them. The probable source of heat for this thermal disturbance is the microporphyritic intrusion encountered at the bottom of the hole, and its age establishes that the major fault activity must have occurred prior to $102.3+0.5 \mathrm{Ma}$ (middle Cretaceous).

Geophysical logs run in the saturated and unsaturated sections of the borehole were invaluable for interpretation of stratigraphy and structure. Problems encountered during logging were lack of service tables for stacked logs, lack of calibration tables for the ER-12-1 hole size, and lack of written procedures for running these logs in the field.

Hydrologic investigations consisted of water level monitoring, flow logging, aquifer tests, and drill-stem tests. The results indicate that the static composite fluid level in well ER-12-1 was 469 $\mathrm{m}(1540 \mathrm{ft})$ below land surface. Drill-stem tests and flow logs determined that the lower two intervals in the well are underpressured relative to the upper zones by approximately $396 \mathrm{~m}(1300 \mathrm{ft})$. Aquifer tests, drill-stem tests, and flow logs determined that the transmissivity of the well ranged from 7.5 $\times 10^{-6}$ to $4 \times 10^{-4} \mathrm{~m}^{2} / \mathrm{s}$, with the most transmissive zone being 518 to $555 \mathrm{~m}(1700$ to $1820 \mathrm{ft}$ ) below land surface followed by the 914 to $963 \mathrm{~m}$ ( 3000 to $3160 \mathrm{ft}$ ). The pressure differential between these zones allowed for substantial crossflow to occur while the well was open.

Two types of geochemical samples were acquired from this well. Water quality samples taken during drilling and testing indicated very few problems associated with the well. Those identified consisted of elevated quantities of volatile and semivolatile organics (sampie 10006) and metals (sample 10007) associated with the drilling process. Geochemical characterization samples were taken only from the uppermost zone, 518 to $555 \mathrm{~m}$ (1700 to $1820 \mathrm{ft}$ ). The results from this sample 
indicate anomalous chemistry, which is most likely due to residual drilling fluids contaminating the sample. Trace quantities of tritium were detected. Origin of the tritium was hypothesized to be from residual drilling fluids entrained in the well, from tritiated surface water that infiltrated in the nearby tunnel ponds, or, less likely, from the underground nuclear tests conducted within Rainier Mesa. Existing data does not differentiate between the various hypotheses. 


\section{INTRODUCTION}

\section{Objective of ER-12-1}

The objective of drillhole ER-12-1 was to determine the hydrogeology of Paleozoic carbonate rocks and of the Eleana Formation, a regional aquitard, in an area potentially down-gradient from underground nuclear testing conducted in nearby Rainier Mesa.

\section{Surficial Setting}

Coordinates for ER-12-1 were surveyed as N886,640.26 ft E640,538.85 ft (Nevada Central Coordinates). This location is in Area 12 of the Nevada Test Site (NTS) (Figure 1) near the base of the eastern slope of Rainier Mesa, alongside the U12e tunnel access road where it passes the base of Dolomite Hill. The drill site is at an elevation of $1773.51 \mathrm{~m}(5817.12 \mathrm{ft})$ and is collared in a thin veneer of alluvium that overlies the Sevy Dolomite. It is approximately $610 \mathrm{~m}(2000 \mathrm{ft})$ northwest of the surficial expression of the Tongue Wash Fault, a northeast-trending sinistral-reverse fault that dips approximately $45^{\circ}$ to the west.

Surface water from the ER-12-1 site drains into Tongue Wash, which eventually flows into other ephemeral channels draining east into Yucca Flat.

\section{Geologic Setting}

The ER-12-1 borehole location was chosen to allow penetration of dolomite for one to two hundred meters below the water table, and to reach Eleana Formation in the footwall of the Tongue Wash fault within the $1070 \mathrm{~m}$ ( $3500 \mathrm{ft}$ ) design depth (Cole, unpublished). A detailed prediction of the geology beneath ER-12-1 is contained in Cole (unpublished), which has been included herein as Appendix A.

\section{Hydrologic Setting}

The ER-12-1 drill site is located within the Ash Meadows groundwater subbasin defined by Winograd and Thordarson (1975) and Waddell et al. (1984). Interpretations based upon Winograd and Thordarson's (1975) subbasin boundary have groundwater beneath ER-12-1 ultimately discharging at Ash Meadows and perhaps Death Valley. A detailed prediction of the ER-12-1 hydrogeology is contained in Russell (1991, unpublished), which has been included in this report as Appendix B.

\section{Location with Respect to Testing}

The drill site for ER-12-1 is located approximately $2.1 \mathrm{~km}(1.3 \mathrm{mi})$ southeast and east of underground nuclear tests conducted within Rainier Mesa. Some of these tests were conducted in a saturated groundwater lens that is perched/semiperched a maximum of $600 \mathrm{~m}(2000 \mathrm{ft})$ above the regional groundwater table (Thordarson, 1965). The drill site is also located $460 \mathrm{~m}(1500 \mathrm{ft}$ ) east of the inactive U12e tunnel effluent ponds. These ponds contained tritiated effluent while the tunnel was active, and since they were unlined ponds, some of the tritiated fluid may have entered the underlying carbonate rocks. 


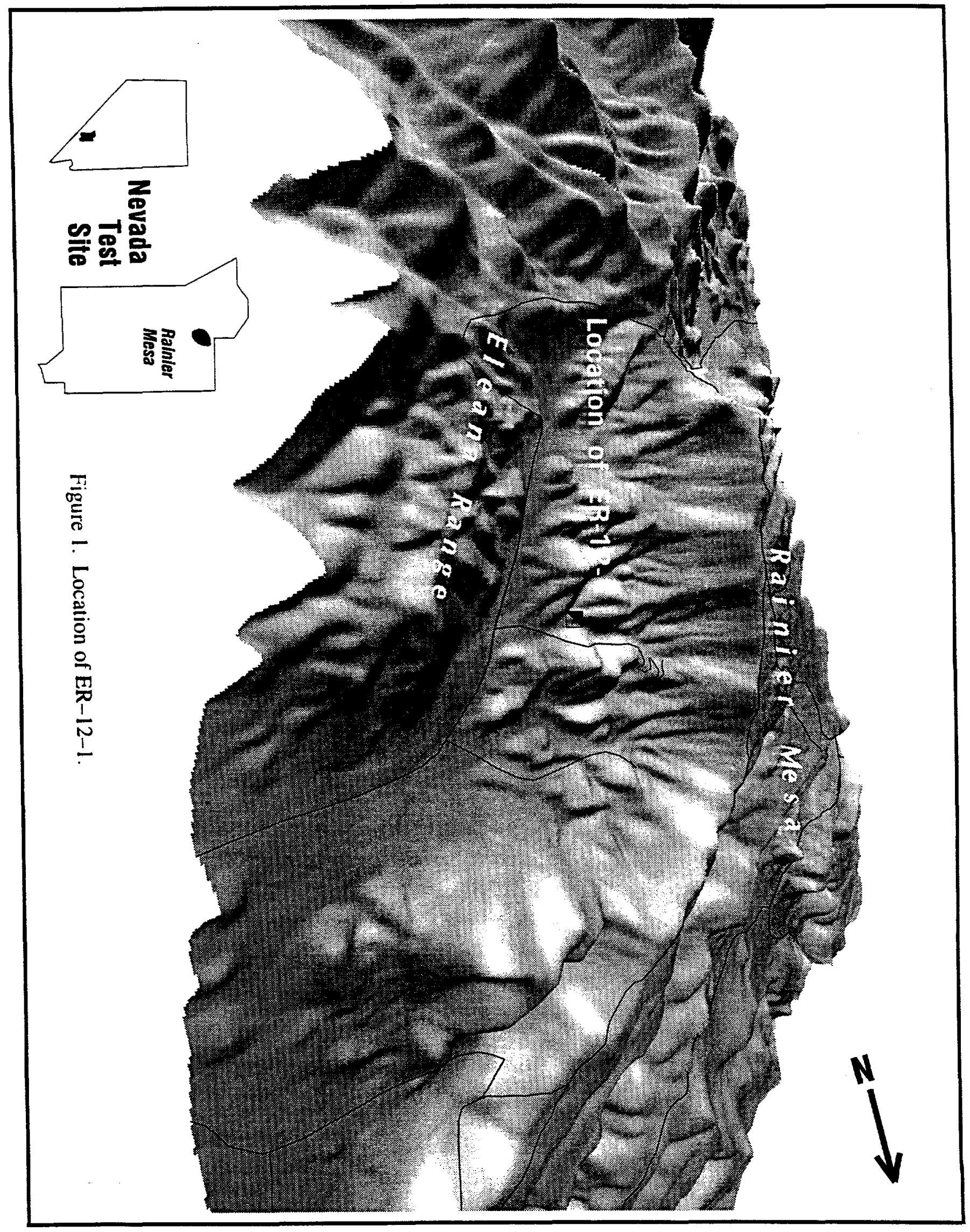




\section{Previous Investigations}

The Dolomite Hill hole, drilled in 1959, is located approximately $580 \mathrm{~m}$ (1900 ft) west of the ER-12-1 drillhole. The hole penetrated $365 \mathrm{~m}$ (1200 ft) of unsaturated dolomite. The lithology of the borehole and a geologic map of the surrounding area were published by Dickey and McKeown (1959). A detailed, 1:24,000 scale geologic map of the Rainier Mesa quadrangle was published by Gibbons et al. (1963). U12e.M-1, an exploratory corehole, was drilled within the U12e tunnel complex, spudding at an elevation of $1877 \mathrm{~m}$ (6158 ft). This borehole encountered $297 \mathrm{~m}(974 \mathrm{ft})$ of Tertiary volcanics and $161 \mathrm{~m}(527 \mathrm{ft})$ of Paleozoic dolomites. A detailed lithologic $\log$ from this borehole was reported by Schoff and Winograd (1961) along with some preliminary results of hydrologic investigations of this and other carbonate-penetrating boreholes. Three additional boreholes (U12e.06A, U12e.06B, and U12e.06R/C) penetrated the Paleozoic dolomites in the area of ER-12-1 (Miller, 1970). The hydrogeology of some of the aforementioned boreholes were included in Thordarson's (1965) report on the hydrogeology of Rainier Mesa.

Over 90 boreholes, listed in Table 1, have been drilled within $2590 \mathrm{~m}(8500 \mathrm{ft})$ of ER-12-1 (Drellack et al., 1991). The majority of these boreholes were completed in Tertiary volcanics and provide only limited information relevant to the conditions at ER-12-1. The unpublished reports of Cole and Russell (Appendix A and Appendix B, respectively) represent the most recent compilation and interpretation of the geology and hydrology of the area surrounding ER-12-1.

\section{PRE-DRILLING ACTIVITIES}

\section{Environmental Surveys}

Three types of surveys, land, cultural resources, and biological, were conducted prior to ER-12-1 drill pad and access road construction. Land surveying activities began on March 30, 1990 with an initial survey of a site $300 \mathrm{~m}(1000 \mathrm{ft})$ east of the current location. The original location was moved approximately $300 \mathrm{~m}(1000 \mathrm{ft})$ to the west to ensure the presence of saturated dolomites at depth. A survey of the new location was conducted on July 16, 1990. Two additional, yet minor relocations of the drill site were conducted on August 2, 1990 and September 24, 1990 to accommodate construction criteria associated with the placement of the drill rig on location. Final as-built-coordinates of the wellhead are (N886,640.26 E640,538.85 ft) Nevada Central Coordinates. The ground level elevation at the wellhead is $1773.51 \mathrm{~m}(5817.12 \mathrm{ft})$.

Cultural resources surveys of the ER-12-1 drill pad and construction sites were conducted by Desert Research Institute (DRI) personnel on August 29, 1990. The results of the survey determined that much of the area encompassed by the drill site had been disturbed by previous construction and use as an equipment yard. A two-track road runs along the western side of the site location, and entrenched drainage runs along the eastern margin. No cultural resources predating the construction and use of the equipment yard were identified during the survey (DRI, 1990).

Biological surveys of the ER-12-1 drill pad and construction site were conducted by EG\&G personnel on August 29, 1990. The results of the survey indicated Blackbrush (Coleogyne 
Table 1. Drill Holes and Tunnels within $2590.8 \mathrm{~m}$ (8500 ft) Radius of ER-12-1 (Revised from Drellack et al., 1991 to account for updated ER-12-1 coordinates).

\begin{tabular}{|c|c|c|c|c|c|c|}
\hline \multirow[b]{2}{*}{ HOLE / TUNNEL NAME } & \multirow[b]{2}{*}{ NORTH } & \multicolumn{3}{|c|}{$\begin{array}{l}\text { DISPLACEMENT AND BEARING } \\
\text { FROM EMP. HOLE TO TARGET }\end{array}$} & \multirow[b]{2}{*}{ BEARING } & \multirow[b]{2}{*}{ EW } \\
\hline & & EAST & DISP-FT & NS & & \\
\hline Mac Exploratory Co. \#1 & 886,712 - & 638,632 & 1908.4 & $\mathbf{N}$ & 87.8 & $\mathrm{~W}$ \\
\hline Effinger \#2 & $886,283-$ & 643,089 & 2574.9 & $\mathrm{~S}$ & 82.0 & $\mathrm{E}$ \\
\hline U-12e Tunnel & $887,459-$ & 637,961 & 2705.0 & $\mathrm{~N}$ & 72.4 & $\mathrm{~W}$ \\
\hline Effinger \#4 & $886,025-$ & 643,216 & 2746.7 & $\mathbf{S}$ & 77.1 & $\mathrm{E}$ \\
\hline U-12es Tunnel & $887,420-$ & 637,808 & 2840.2 & $\mathbf{N}$ & 74.1 & W \\
\hline U-12e Tunnel Water Conduit & 887,342 - & 637,527 & 3092.7 & $\mathrm{~N}$ & 76.9 & $\mathrm{~W}$ \\
\hline$U-12 b \# 4$ & $889,683-$ & 636,773 & 4841.8 & $\mathbf{N}$ & 51.1 & W \\
\hline U-12e.02-J & 886,350 & 635,608 & 4939.5 & $\mathrm{~S}$ & 86.6 & W \\
\hline U-12b Tunnel & 889,759 & 636,481 & 5118.2 & $\mathrm{~N}$ & 52.5 & W \\
\hline U-12f Tunnel & $889,667-$ & 636,115 & 5360.5 & $\mathbf{N}$ & 55.6 & W \\
\hline U-12c Tunnel & $889,768-$ & 636,138 & 5399.4 & $\mathrm{~N}$ & 54.6 & W \\
\hline U-12d Tunnel & 889,615 & 636,033 & 5399.5 & $\mathrm{~N}$ & 56.6 & W \\
\hline $\mathrm{UE}-12 \mathrm{n} \# 5$ & $890,127-$ & 636,331 & 5465.0 & $\mathrm{~N}$ & 50.4 & W \\
\hline $\mathrm{U}-12 \mathrm{~b} \# 2$ & $889,947-$ & 636,111 & 5526.6 & $\mathbf{N}$ & 53.3 & W \\
\hline $\mathrm{U}-12 \mathrm{~b} \# 1$ & $890,432-$ & 636,186 & 5773.0 & $\mathrm{~N}$ & 48.9 & W \\
\hline U-12n Ext. Tunnel & 892,487 & 638,583 & 6165.5 & $\mathrm{~N}$ & 18.5 & W \\
\hline U-12e.04-1 Vent & 885,782 & 634,316 & 6281.9 & $\mathbf{S}$ & 82.2 & W \\
\hline U-12g.08 Tunnel & 881,027 & 637,708 & 6286.5 & $\mathrm{~s}$ & 26.8 & W \\
\hline U-12g Tunnel & 881,027 & 637,708 & 6286.5 & $S$ & 26.8 & W \\
\hline U12n Tunnel & 892,667 & 638,645 & 6317.6 & $\mathrm{~N}$ & 17.4 & W \\
\hline U-12n.10A Reentry Mining & 892,739 & 638,485 & 6435.6 & $\mathrm{~N}$ & 18.6 & W \\
\hline U-12e.04 \#22 & 885,163 & 634,216 & 6493.2 & $S$ & 76.9 & W \\
\hline U-12e.05 Hagestad \#3 & $887,086-$ & 634,009 & 6545.2 & $\mathrm{~N}$ & 86.1 & W \\
\hline $\mathrm{U}-12 \mathrm{e} \mathrm{CH} \# 1$ & 886,112 & 633,885 & 6674.9 & $\mathrm{~s}$ & 85.5 & W \\
\hline $\mathrm{U}-12 \mathrm{e} \mathrm{CH} \# 3$ & 886,073 - & 633,888 & 6675.1 & $\mathrm{~S}$ & 85.1 & W \\
\hline $\mathrm{U}-12 \mathrm{e} \mathrm{CH} \# 2$ & 886,092 & 633,886 & 6675.5 & $\mathrm{~S}$ & 85.3 & W \\
\hline U-12b R-4 & $890,571-$ & 635,027 & 6770.1 & $\mathrm{~N}$ & 54.5 & W \\
\hline Unidentified & $884,755-$ & 634,033 & 6773.6 & $\mathrm{~S}$ & 73.8 & W \\
\hline$U-12 b$ R-8 & 890,551 - & 635,003 & 6778.1 & $\mathrm{~N}$ & 54.8 & W \\
\hline U-12b.04A Vent & 890,271 & 634,814 & 6779.4 & $\mathrm{~N}$ & 57.6 & W \\
\hline $\mathrm{U}-12 \mathrm{~b}$ R-7 & 890,561 - & 635,003 & 6783.9 & $\mathrm{~N}$ & 54.7 & W \\
\hline$U-12 b$ R-3 & 890,573 & 635,006 & 6788.4 & $\mathrm{~N}$ & 54.6 & W \\
\hline U-12b R-2 & $890,571-$ & 635,003 & 6789.7 & $\mathrm{~N}$ & 54.6 & W \\
\hline U-12b R-1 & $890,570-$ & 635,000 & 6791.6 & $\mathrm{~N}$ & 54.6 & W \\
\hline U-12b R-5 & $890,574-$ & 635,000 & 6793.9 & $\mathrm{~N}$ & 54.6 & W \\
\hline U-12b R-9 & 890,596 - & 635,003 & 6804.2 & $\mathrm{~N}$ & 54.5 & W \\
\hline U-12b R-6 & $890,581-$ & 634,992 & 6804.5 & $\mathrm{~N}$ & 54.6 & W \\
\hline U-12e.04 \#21 & $884,683-$ & 634,012 & 6814.1 & $S$ & 73.3 & $\mathrm{~W}$ \\
\hline U-12e.04 \#32 (USGS) & $884,669-$ & 634,015 & 6815.2 & $\mathrm{~S}$ & 73.2 & W \\
\hline U-12b Unknown \#1 & 890,574 & 634,971 & 6817.5 & $\mathrm{~N}$ & 54.8 & $\mathrm{~W}$ \\
\hline U-12e.04 \#24 & $884,675-$ & 634,006 & 6822.1 & $\mathrm{~S}$ & 73.3 & W \\
\hline Unidentified & $884,441-$ & 634,020 & 6879.9 & $S$ & 71.4 & W \\
\hline$U-12 b \# 3$ & 890,617 & 634,913 & 6889.7 & $\mathrm{~N}$ & 54.7 & W \\
\hline Unidentified & 884,404 & 634,013 & 6898.4 & $S$ & 71.1 & W \\
\hline Unidentified & $884,395-$ & 634,014 & 6900.4 & $\mathrm{~S}$ & 71.0 & W \\
\hline U-12e.03aa PS & $887,716-$ & 633,675 & 6947.8 & $\mathrm{~N}$ & 81.1 & W \\
\hline U-12b.04-1 & $890,215-$ & 634,558 & 6968.0 & $\mathrm{~N}$ & 59.1 & $\mathrm{~W}$ \\
\hline U12b.04C Sample \& Vent & 890,230 & 634,554 & 6979.1 & $\mathrm{~N}$ & 59.0 & $\mathrm{~W}$ \\
\hline$U-12 b .04-2$ & $890,250-$ & 634,558 & 6986.0 & $\mathrm{~N}$ & 58.9 & W \\
\hline$U-12 b .04 B$ & 890,232 & 634,545 & 6987.9 & $\mathrm{~N}$ & 59.1 & W \\
\hline
\end{tabular}


Table 1. Drill Holes and Tunnels within $2590.8 \mathrm{~m}$ (8500 ft) Radius of ER-12-1 (Drellack et al., 1991) (continued).

\begin{tabular}{|c|c|c|c|c|c|c|}
\hline HOLE NAME & NORTH & EAST & DISP-FT & NS & BEARING & EW \\
\hline $\mathrm{U}-12 \mathrm{~b} .04-4$ & 890,215 - & 634,523 & 6998.1 & $\mathrm{~N}$ & 59.3 & W \\
\hline U-12e.M1 & $886,644-$ & 633,532 & 7007.0 & $\mathrm{~N}$ & 90.0 & W \\
\hline U-12e.03ac PS & 887,652 & 633,598 & 7014.4 & $\mathrm{~N}$ & 81.7 & $\mathrm{~W}$ \\
\hline U-12b.04-3 & $890,250-$ & 634,523 & 7016.0 & $\mathrm{~N}$ & 59.0 & W \\
\hline U-12e.05 Hagestad \#4 & 887,609 & 633,451 & 7153.9 & $\mathrm{~N}$ & 82.2 & W \\
\hline U-12b.04 PS & 890,101 - & 634,271 & 7160.1 & $\mathrm{~N}$ & 61.1 & W \\
\hline$U-12 b .04-5$ & $890,123-$ & 634,261 & 7179.5 & $\mathrm{~N}$ & 61.0 & W \\
\hline U-12b Unknown \#2 & 890,128 & 634,256 & 7186.3 & $\mathrm{~N}$ & 61.0 & W \\
\hline UE- $12 \mathrm{n} \# 7$ & $892,004-$ & 635,755 & 7187.4 & $\mathrm{~N}$ & 41.7 & W \\
\hline UE-12n \#4 & 892,035 & 635,753 & 7211.9 & $\mathrm{~N}$ & 41.6 & W \\
\hline U-12g.01-1 Vent & 881,814 & 635,178 & 7213.2 & $S$ & 48.0 & W \\
\hline $\mathrm{U}-12 \mathrm{~g} .07 \mathrm{Ch} \# 1$ & $883,336-$ & 633,951 & 7370.1 & $S$ & 63.4 & W \\
\hline U-12e.04 \#29S & $883,636-$ & 633,731 & 7441.3 & $\mathrm{~S}$ & 66.2 & W \\
\hline U-12e. $04 \# 29$ & $883,638-$ & 633,728 & 7443.2 & $S$ & 66.2 & W \\
\hline U-12b.09a PS & $890,023-$ & 633,880 & 7469.1 & $\mathrm{~N}$ & 63.1 & W \\
\hline U-12b.09-1 Vent & $890,621-$ & 634,188 & 7495.6 & $\mathrm{~N}$ & 57.9 & W \\
\hline $\mathrm{U}-12 \mathrm{~b} .09 \mathrm{~d}$ PS & 889,979 - & 633,826 & 7497.6 & $\mathrm{~N}$ & 63.6 & W \\
\hline U-12b.08-1 Vent & $890,795-$ & 634,110 & 7654.8 & $\mathrm{~N}$ & 57.1 & W \\
\hline U-12b.07-1 & 891,222 - & 634,404 & 7657.2 & $\mathrm{~N}$ & 53.2 & W \\
\hline U-12g.07 Tunnel & $883,930-$ & 633,350 & 7682.8 & $S$ & 69.4 & W \\
\hline U-12g.07 PS \#1V & 883,930 & 633,350 & 7682.8 & $S$ & 69.4 & W \\
\hline U-12e.03ab PS & 887,849 & 632,911 & 7723.2 & $N$ & 81.0 & W \\
\hline U-12e.03a-BS Ps & $887,849-$ & 632,911 & 7723.2 & $\mathrm{~N}$ & 81.0 & W \\
\hline U-12n.07 Tunnel & 892,249 - & 634,984 & 7894.2 & $\mathrm{~N}$ & 44.7 & W \\
\hline U-12b.08-3 PSb.08-4 PS & $890,265-$ & 633,514 & 7905.1 & $\mathrm{~N}$ & 62.7 & W \\
\hline U-12b.08-2 R/C & 890,256 & 633,509 & 7905.5 & $\mathrm{~N}$ & 62.8 & W \\
\hline U-12e.10 PS \#1V & 886,497 & 632,609 & 7931.3 & $\mathrm{~S}$ & 89.0 & W \\
\hline U-12e.10 Tunnel & 886,497 & 632,609 & 7931.3 & $S$ & 89.0 & W \\
\hline U-12b.08-4 PS W/S & $890,182-$ & 633,442 & 7931.8 & $\mathrm{~N}$ & 63.5 & W \\
\hline U-12b.09b PPS & $890,328-$ & 633,505 & 7942.2 & $\mathrm{~N}$ & 62.3 & W \\
\hline U-12e.11 Tunnel & 884,808 & 632,786 & 7966.5 & $\mathrm{~S}$ & 76.7 & W \\
\hline U-12e.11 PPS \#1V & $884,808-$ & 632,786 & 7966.5 & $S$ & 76.7 & W \\
\hline U-12g.06 Tunnel & 882,815 & 633,526 & 7988.3 & $\mathrm{~S}$ & 61.4 & $\mathrm{~W}$ \\
\hline U-12b.07-3 Vent & $891,533-$ & 634,217 & 7994.3 & $\mathrm{~N}$ & 52.3 & W \\
\hline U-12e.07-6 Vent & $887,478-$ & 632,578 & 8005.0 & $\mathrm{~N}$ & 84.0 & W \\
\hline U-12g.06 PS \#1V & 882,792 & 633,516 & 8008.1 & $\mathrm{~S}$ & 61.3 & W \\
\hline U-12e.17 Tunnel & $885,289-$ & 632,494 & 8157.6 & $\mathrm{~S}$ & 80.5 & $\mathrm{~W}$ \\
\hline U-12n.09 Tunnel & 892,520 & 634,765 & 8241.0 & $\mathrm{~N}$ & 44.5 & W \\
\hline U-12e.16 Tunnel & $885,250-$ & 632,401 & 8255.9 & $S$ & 80.3 & W \\
\hline U-12e.15 Tunnel & $885,204-$ & 632,312 & 8351.4 & $\mathrm{~S}$ & 80.1 & W \\
\hline UE-12g. $10 \# 3$ & $882,944-$ & 633,034 & 8365.7 & $\mathrm{~S}$ & 63.8 & W \\
\hline $\mathrm{U}-12 \mathrm{n} .0 \mathrm{l}-2 \mathrm{R} / \mathrm{C}$ & 893,208 & 635,333 & 8381.0 & $\mathrm{~N}$ & 38.4 & W \\
\hline U-12b.10-2 PS W/S & 890,915 & 633,328 & 8383.0 & $\mathrm{~N}$ & 59.3 & W \\
\hline U-12n.01-4 PPS & 893,116 & 635,214 & 8384.2 & $\mathrm{~N}$ & 39.4 & W \\
\hline U-12n.01-3 PPS & $893,206-$ & 635,322 & 8386.3 & $\mathrm{~N}$ & 38.5 & $\mathrm{~W}$ \\
\hline U-12b. $10-5$ PS & 890,853 & 633,258 & 8412.0 & $\mathrm{~N}$ & 59.9 & $\mathrm{~W}$ \\
\hline $\mathrm{U}-12 \mathrm{~b} \cdot 10-1 \mathrm{R} / \mathrm{C}$ & $890,853-$ & 633,248 & 8420.7 & $\mathrm{~N}$ & 60.0 & $\mathrm{~W}$ \\
\hline U-12b.10-4 PS & 890,854 & 633,238 & 8429.9 & $\mathrm{~N}$ & 60.0 & W \\
\hline $\mathrm{U}-12 \mathrm{n} .01-5 \mathrm{CH}$ & 893,374 - & 635,463 & 8432.8 & $\mathrm{~N}$ & 37.0 & W \\
\hline U-12e.20 Tunnel & $887,646-$ & 632,161 & 8438.2 & $\mathrm{~N}$ & 83.2 & W \\
\hline U-12g.10 Tunnel & $883,643-$ & 632,607 & 8479.3 & $\mathrm{~S}$ & 69.3 & W \\
\hline U-12g.10 Reentry Mining & 883,643 & 632,607 & 8479.3 & $S$ & 69.3 & W \\
\hline U-12n.06 PS \#1 & 892,551 & 634,459 & 8479.8 & $\mathrm{~N}$ & 45.8 & $\mathrm{~W}$ \\
\hline
\end{tabular}

Data, including hole nomenclature, from RSN Nevada Test Site Drilling and Mining Summary

*Mac Exnloratorv is equivalent to "Dolomite Hill" 
ramosissima) dominates the floral community. Smaller amounts of sagebrush (Artemisia nova), little rabbitbrush (Chrysothamnus spp.), four-wing saltbush (Atriplex canescens), cliffrose (Cowania mexicana), Mormon tea (Ephedra nevadensis), and rice grass (Oryzopsis hymenoides) were also present. One hawk and one lizard were seen. Rabbit scat was noted in the area, but no rabbits were observed (DRI, 1990).

\section{Site Preparation}

Environmental surveys were followed by drill pad and access road construction. Construction was initiated on May 28, 1991 and completed on June 5, 1991. The original mud pit was enlarged on June, 19, 20, and 21 and was lined with sand and a polypropylene liner on July 11, 1991.

\section{DRILLING TECHNIQUES AND HOLE HISTORY}

ER-12-1 was drilled using the Cardwell 500 rotary drill rig. The hole was spudded on July 19 , 1991 , and reached a total depth of $1093.6 \mathrm{~m}$ (3588 ft) on October 17, 1991. During construction of the hole, surface casing with an outside diameter of $0.51 \mathrm{~m}$ (20 in) was set to a depth of $15.8 \mathrm{~m}$ (52 $\mathrm{ft}$ ), and an intermediate casing string $0.34 \mathrm{~m} \mathrm{(13} \mathrm{3/8} \mathrm{in)} \mathrm{in} \mathrm{diameter} \mathrm{was} \mathrm{placed} \mathrm{from} \mathrm{the} \mathrm{surface} \mathrm{to}$

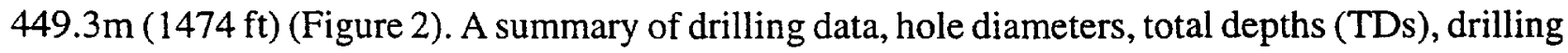
techniques, and casing records for ER-12-1 is contained in Appendix C of this report.

Drilling techniques employed at ER-12-1 included rotary tri-cone and rotary hammer drilling with conventional and reverse circulation using air/water, air/foam (Davis mix), or bentonite mud. Four cores were also obtained at various depths in the borehole. Table 2 lists the specific techniques used in drilling this hole.

Twenty-six drill bits of various types and diameter were used in drilling ER-12-1. These bit types included tri-cone button and tooth bits, hammer bits, and coring bits. The diameter, manufacturer, footage drilled, dates, and formation drilled for each bit used in ER-12-1 are summarized in Table 3. The large variety of drill bits and drilling techniques were due to experimentation with optimizing penetration rates in carbonate rocks.

Single-shot hole deviation surveys were run throughout the drilling of ER-12-1. Additionally, following the completion of drilling, a gyroscopic hole deviation survey was obtained by Schlumberger Formation Micro-scanner survey, which recorded hole deviation data over the saturated portion of the borehole. Selected survey depths, amount of deviation, and the date the surveys were conducted are listed in Table 4.

The first six surveys conducted by Reynolds Electrical Engineering Co., Inc. (REECo) during drilling were not accurate as the survey tool was run in an inverted position. The hole deviation survey record for ER-12-1 obtained by REECo during drilling indicates the hole to be apparently straight with fairly consistent deviation survey readings of less than $2^{\circ}$. However, the hole deviation survey obtained by Schlumberger (Formation Micro-scanner) following completion of the drilling indicated a significant increase in hole deviation below approximately $610 \mathrm{~m}(2000 \mathrm{ft})$. The 


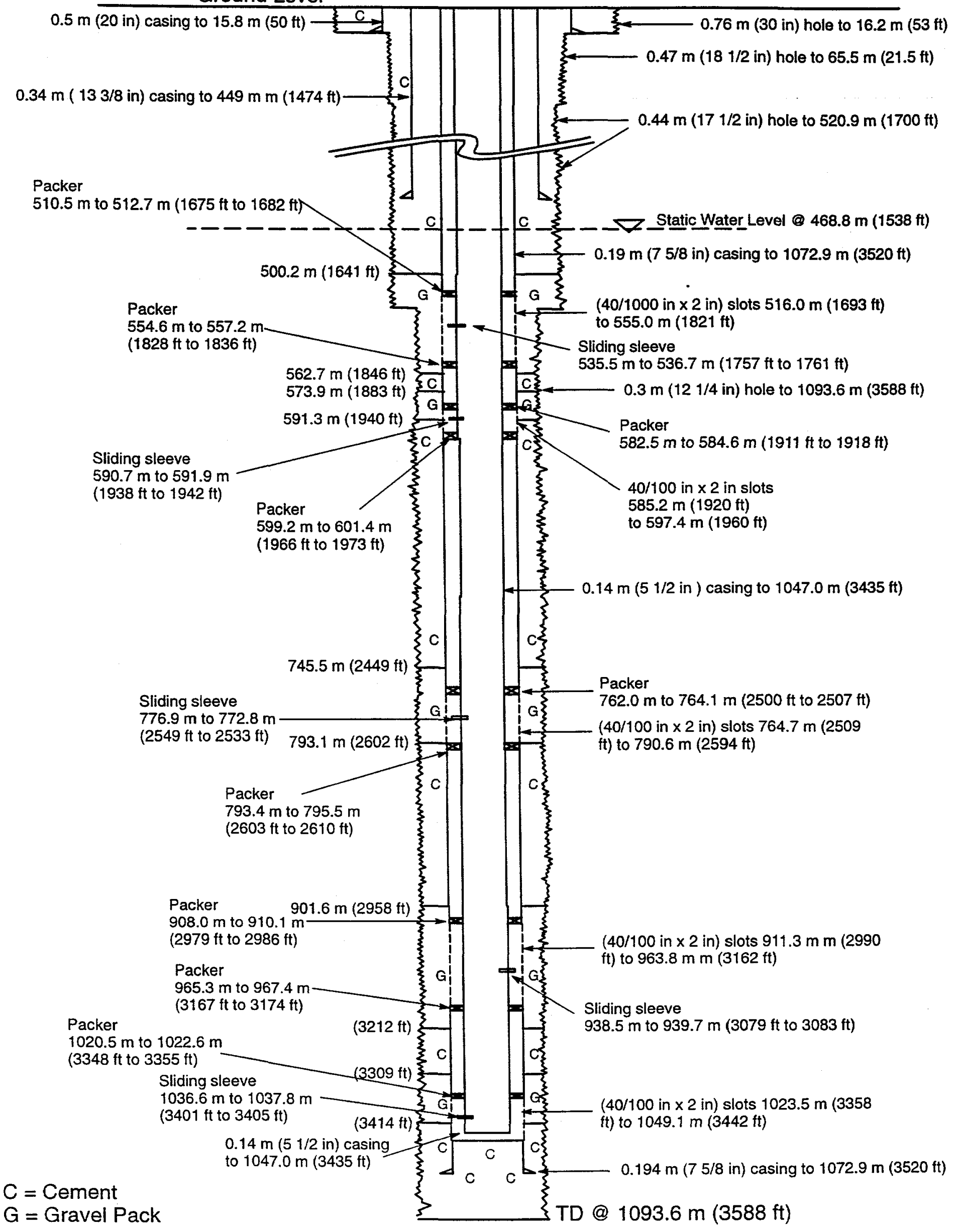

Figure 2. Schematic of ER-12-1 hole construction. 
Table 2. Drilling Techniques used at ER-12-1.

\begin{tabular}{|c|c|}
\hline Depth Interval & Drilling Technique \\
\hline $\begin{array}{l}0-16.2 \mathrm{~m} \\
(0-53 \mathrm{ft})\end{array}$ & $\begin{array}{l}\text { Rotary drilled surface hole using mud and } \\
\text { conventional circulation }\end{array}$ \\
\hline $\begin{array}{l}16.2-30.5 \mathrm{~m} \\
(53-100 \mathrm{ft})\end{array}$ & $\begin{array}{l}\text { Rotary drilled a } 0.47 \mathrm{~m}(18-1 / 2 \mathrm{in}) \text { hole using } \\
\text { water and conventional circulation }\end{array}$ \\
\hline $\begin{array}{l}30.5-65.5 \mathrm{~m} \\
(100-215 \mathrm{ft})\end{array}$ & $\begin{array}{l}\text { Rotary drilled using air/water and reverse } \\
\text { circulation }\end{array}$ \\
\hline $\begin{array}{l}65.5-520.9 \mathrm{~m} \\
(215-1709 \mathrm{ft})\end{array}$ & $\begin{array}{l}\text { Rotary drilled a } 0.445 \mathrm{~m}(17-1 / 2 \mathrm{in}) \text { hole using } \\
\text { an air hammer bit with conventional circulation and } \\
\text { air/foam }\end{array}$ \\
\hline $\begin{array}{l}520.9-539.2 \mathrm{~m} \\
(1709-1769 \mathrm{ft})\end{array}$ & $\begin{array}{l}\text { Rotary drilled a } 0.311 \mathrm{~m}(12-1 / 4 \mathrm{in}) \text { hole using } \\
\text { conventional circulation and air/foam }\end{array}$ \\
\hline $\begin{array}{l}539.2-543.8 \mathrm{~m} \\
(1769-1784 \mathrm{ft})\end{array}$ & $\begin{array}{l}\text { Rotary cored using a } 22.2 \mathrm{~cm}(8-3 / 4 \mathrm{in}) \text { diamond } \\
\text { core bit (Core \#1); reamed to } 0.311 \mathrm{~m}(12-1 / 4 \text { in) }\end{array}$ \\
\hline $\begin{array}{l}543.8-548.0 \mathrm{~m} \\
(1784-1798 \mathrm{ft})\end{array}$ & $\begin{array}{l}\text { Rotary drilled a } 0.311 \mathrm{~m}(12-1 / 4 \text { in) hole using } \\
\text { dual-string reverse circulation and air/foam }\end{array}$ \\
\hline $\begin{array}{l}548.0-556.3 \mathrm{~m} \\
(1798-1825 \mathrm{ft})\end{array}$ & $\begin{array}{l}\text { Rotary cored using a } 21.6 \mathrm{~cm}(8-1 / 2 \mathrm{in}) \text { four-cone } \\
\text { bit and marine core barrel (Core \#2); reamed to } 0.311 \mathrm{~m} \\
(12-1 / 4 \mathrm{in})\end{array}$ \\
\hline $\begin{array}{l}556.3-556.9 \mathrm{~m} \\
(1825-1827 \mathrm{ft})\end{array}$ & $\begin{array}{l}\text { Rotary drilled a } 0.311 \mathrm{~m}(12-1 / 4 \mathrm{in}) \text { hole using } \\
\text { conventional circulation and air/foam }\end{array}$ \\
\hline $\begin{array}{l}556.9-751.0 \mathrm{~m} \\
(1827-2464 \mathrm{ft})\end{array}$ & $\begin{array}{l}\text { Rotary drilled a } 0.311 \mathrm{~m}(12-1 / 4 \mathrm{in}) \text { hole using } \\
\text { conventional circulation and bentonite mud }\end{array}$ \\
\hline $\begin{array}{l}751.0-757.7 \mathrm{~m} \\
(2464-2486 \mathrm{ft})\end{array}$ & $\begin{array}{l}\text { Rotary cored using a } 21.6 \mathrm{~cm}(8-1 / 2 \mathrm{in}) \text { four-cone } \\
\text { bit and marine core barrel (Core \#3); } \\
\text { reamed to } 0.311 \mathrm{~m}(12-1 / 4 \mathrm{in})\end{array}$ \\
\hline $\begin{array}{l}757.7-895.2 \mathrm{~m} \\
(2486-2937 \mathrm{ft})\end{array}$ & $\begin{array}{l}\text { Rotary drilled a } 0.311 \mathrm{~m}(12-1 / 4 \mathrm{in}) \text { hole using } \\
\text { conventional circulation and bentonite mud }\end{array}$ \\
\hline $\begin{array}{l}895.2-903.1 \mathrm{~m} \\
(2937-2963 \mathrm{ft})\end{array}$ & $\begin{array}{l}\text { Rotary cored using a } 22.2 \mathrm{~cm}(8-3 / 4 \mathrm{in}) \text { diamond } \\
\text { core bit (Core \#4); reamed to } 0.311 \mathrm{~m}(12-1 / 4 \text { in) }\end{array}$ \\
\hline $\begin{array}{l}903.1-1093.6 \mathrm{~m} \\
(2963-3588 \mathrm{ft})\end{array}$ & $\begin{array}{l}\text { Rotary drilled a } 0.311 \mathrm{~m} \text { ( } 12-1 / 4 \mathrm{in}) \text { hole using } \\
\text { conventional circulation and bentonite mud }\end{array}$ \\
\hline
\end{tabular}


Table 3. Bit Records for ER-12-1.

\begin{tabular}{|c|c|c|c|c|c|c|c|c|c|}
\hline $\begin{array}{l}\text { Bit } \\
\text { No. }\end{array}$ & $\begin{array}{l}\text { Bit } \\
\text { Diameter }\end{array}$ & $\begin{array}{l}\text { Bit } \\
\text { Type }\end{array}$ & Manufacturer & Date In & $\begin{array}{l}\text { Depth Interval } \\
\text { Drilled }\end{array}$ & $\begin{array}{l}\text { Footage } \\
\text { Drilled }\end{array}$ & $\begin{array}{l}\text { Hours } \\
\text { Drilled }\end{array}$ & $\begin{array}{l}\text { Lithology(s) } \\
\text { Drilled }\end{array}$ & $\begin{array}{l}\text { Comments/ } \\
\text { Bit Grade }\end{array}$ \\
\hline 1 & $\begin{array}{l}66.0 \mathrm{~cm} \\
(26 \mathrm{in})\end{array}$ & $\begin{array}{l}\text { Retip } \\
\text { BTN }\end{array}$ & SEC & $07-19-91$ & $\begin{array}{l}0-6.4 \mathrm{~m} \\
(0-21.0 \mathrm{ft})\end{array}$ & $\begin{array}{l}6.4 \mathrm{~m} \\
(21 \mathrm{ft})\end{array}$ & NA & Alluvium & $\begin{array}{l}\text { Hole not } \\
\text { straight }\end{array}$ \\
\hline 2 & $\begin{array}{l}76.2 \mathrm{~cm} \\
(30 \mathrm{in})\end{array}$ & $\begin{array}{l}\text { Retip } \\
\text { MT }\end{array}$ & STC-Smith & $07-19-91$ & $\begin{array}{l}6.4-16.2 m \\
(21-53 f t)\end{array}$ & $\begin{array}{l}9.8 \mathrm{~m} \\
(32 \mathrm{ft})\end{array}$ & NA & Dolomite & \\
\hline 3 & $\begin{array}{l}47.0 \mathrm{~cm} \\
(18-1 / 2 \mathrm{in})\end{array}$ & BTN & Hughes & $07-24-91$ & $\begin{array}{l}16.2-30.5 \mathrm{~m} \\
(53-100 \mathrm{ft})\end{array}$ & $\begin{array}{l}14.3 \mathrm{~m} \\
(47 \mathrm{ft})\end{array}$ & 22.5 & $\begin{array}{l}\text { Cement } \\
\text { dolomite }\end{array}$ & \\
\hline 4 & $\begin{array}{l}47.0 \mathrm{~cm} \\
(18-1 / 2 \mathrm{in})\end{array}$ & $\begin{array}{l}\text { BTN } \\
44 R\end{array}$ & Hughes & $07-26-91$ & $\begin{array}{l}30.5-65.5 \mathrm{~m} \\
(100-215 \mathrm{ft})\end{array}$ & $\begin{array}{l}35.1 \mathrm{~m} \\
(115 \mathrm{ft})\end{array}$ & 67.5 & Dolomite & \\
\hline 5 & $\begin{array}{l}44.5 \mathrm{~cm} \\
(17-1 / 2 \mathrm{in})\end{array}$ & $\begin{array}{l}\text { Hammer } \\
\text { Q5MJ }\end{array}$ & Smith & $08-07-91$ & $\begin{array}{l}3.4-65.5 \mathrm{~m} \\
(11-215 \mathrm{ft})\end{array}$ & $\begin{array}{l}62.2 \mathrm{~m} \\
(204 \mathrm{ft})\end{array}$ & NA & Cement & $\begin{array}{l}\text { Drilled } \\
\text { casing cement }\end{array}$ \\
\hline 6 & $\begin{array}{l}44.5 \mathrm{~cm} \\
(17-1 / 2 \mathrm{in})\end{array}$ & $\begin{array}{l}\text { BTN } \\
\text { Q5MJ }\end{array}$ & Smith & $08-08-91$ & $\begin{array}{l}65.5-73.2 \mathrm{~m} \\
(215-240 \mathrm{ft})\end{array}$ & $\begin{array}{l}7.6 \mathrm{~m} \\
(25 \mathrm{ft})\end{array}$ & 6 & $\begin{array}{l}\text { Cement/ } \\
\text { dolomite }\end{array}$ & \\
\hline 7 & $\begin{array}{l}44.5 \mathrm{~cm} \\
(17-1 / 2 \mathrm{in})\end{array}$ & $\begin{array}{l}\text { Hammer } \\
\text { SD-12 }\end{array}$ & Smith & 08-09-91 & $\begin{array}{l}73.2-521.0 \mathrm{~m} \\
(240-1709 \mathrm{ft})\end{array}$ & $\begin{array}{r}447.8 \mathrm{~m} \\
(1469 \mathrm{ft})\end{array}$ & 77 & $\begin{array}{l}\text { Dolomite/ } \\
\text { siltstone }\end{array}$ & \\
\hline 8 & $\begin{array}{l}31.1 \mathrm{~cm} \\
(12-1 / 4 \mathrm{in})\end{array}$ & $\begin{array}{l}\text { BTN } \\
\text { J-55 }\end{array}$ & Hughes & $08-26-91$ & $\begin{array}{l}521.0-556.9 \mathrm{~m} \\
(1709-1827 \mathrm{ft})\end{array}$ & $\begin{array}{l}36.0 \mathrm{~m} \\
(118 \mathrm{ft})\end{array}$ & NA & $\begin{array}{l}\text { Dolomite ( \& } \\
1440-1709 \mathrm{ft} \\
\text { sand/cement) }\end{array}$ & \\
\hline $\begin{array}{l}\text { core } \\
\# 1\end{array}$ & $\begin{array}{l}22.2 \mathrm{~cm} \\
(8-3 / 4 \mathrm{in})\end{array}$ & $\begin{array}{l}\text { Conventional } \\
\text { diamond core }\end{array}$ & Christensen & $08-30-91$ & $\begin{array}{l}539.2-543.8 \mathrm{~m} \\
(1769-1784 \mathrm{ft})\end{array}$ & $\begin{array}{l}4.6 \mathrm{~m} \\
(15 \mathrm{ft})\end{array}$ & NA & Dolomite & $\begin{array}{l}6.1 \mathrm{~cm}(.2 \mathrm{ft} \\
\text { recovery) }\end{array}$ \\
\hline $\begin{array}{l}\text { core } \\
\# 2\end{array}$ & $\begin{array}{l}22.2 \mathrm{~cm} \\
(8-3 / 4 \mathrm{in})\end{array}$ & $\begin{array}{l}\text { Marine core } \\
\text { 4-cone }\end{array}$ & NA & $09-03-91$ & $\begin{array}{l}548.0-556.3 \mathrm{~m} \\
(1798-1825 \mathrm{ft})\end{array}$ & $\begin{array}{l}8.2 \mathrm{~m} \\
(27 \mathrm{ft})\end{array}$ & NA & $\begin{array}{l}\text { Dolomite/ } \\
\text { quartzite \&siltstone }\end{array}$ & No recovery \\
\hline 9 & $\begin{array}{l}31.1 \mathrm{~cm} \\
(12-1 / 4 \mathrm{in})\end{array}$ & BTN & Hughes & $09-05-91$ & $\begin{array}{l}556.9-565.1 \mathrm{~m} \\
(1827-1854 \mathrm{ft})\end{array}$ & $\begin{array}{l}8.2 \mathrm{~m} \\
(27 \mathrm{ft})\end{array}$ & NA & $\begin{array}{l}\text { Quartzite \& } \\
\text { siltstone }\end{array}$ & $\begin{array}{l}\text { Bit } 0.5 \text { in } \\
\text { under guage }\end{array}$ \\
\hline 10 & $\begin{array}{l}31.1 \mathrm{~cm} \\
(12-1 / 4 \mathrm{in})\end{array}$ & $\begin{array}{l}\text { BTN } \\
\text { E-55 }\end{array}$ & Hughes & 09-09-91 & $\begin{array}{l}565.1-643.7 \mathrm{~m} \\
(1854-2112 \mathrm{ft})\end{array}$ & $\begin{array}{l}78.6 \mathrm{~m} \\
(258 \mathrm{ft})\end{array}$ & 35.5 & $\begin{array}{l}\text { Quartzite, } \\
\text { siltstone \&shale }\end{array}$ & \\
\hline 11 & $\begin{array}{l}31.1 \mathrm{~cm} \\
(12-1 / 4 \mathrm{in})\end{array}$ & $\begin{array}{l}\text { BTN } \\
\text { HH-55 }\end{array}$ & Hughes & $09-11-91$ & $\begin{array}{l}643.7-704.1 \mathrm{~m} \\
(2112-2310 \mathrm{ft})\end{array}$ & $\begin{array}{l}60.4 \mathrm{~m} \\
(198 \mathrm{ft})\end{array}$ & 32 & $\begin{array}{l}\text { Quartzite, } \\
\text { siltstone \& shale }\end{array}$ & \\
\hline
\end{tabular}


Table 3. Bit Records for ER-12-1 (continued).

\begin{tabular}{|c|c|c|c|c|c|c|c|c|c|}
\hline $\begin{array}{l}\text { Bit } \\
\text { No. }\end{array}$ & $\begin{array}{l}\text { Bit } \\
\text { Diameter }\end{array}$ & $\begin{array}{l}\text { Bit } \\
\text { Type }\end{array}$ & Manufacturer & Date In & $\begin{array}{l}\text { Depth Interval } \\
\text { Drilled }\end{array}$ & $\begin{array}{l}\text { Footage } \\
\text { Drilled }\end{array}$ & $\begin{array}{l}\text { Hours } \\
\text { Drilled }\end{array}$ & $\begin{array}{l}\text { Lithology(s) } \\
\text { Drilled }\end{array}$ & $\begin{array}{l}\text { Comments/ } \\
\text { Bit Grade }\end{array}$ \\
\hline 12 & $\begin{array}{l}31.1 \mathrm{~cm} \\
(12-1 / 4 \mathrm{in})\end{array}$ & $\begin{array}{l}\text { BTM } \\
\mathrm{HH}-44\end{array}$ & Hughes & $09-16-91$ & $\begin{array}{l}704.1-732.7 \mathrm{~m} \\
(2310-2404 \mathrm{ft})\end{array}$ & $\begin{array}{r}28.7 \mathrm{~m} \\
(84 \mathrm{ft})\end{array}$ & 25.5 & $\begin{array}{l}\text { Quartzite } \\
\text { siltstone \& shale }\end{array}$ & \\
\hline 13 & $\begin{array}{l}31.1 \mathrm{~cm} \\
(12-1 / 4 \mathrm{in})\end{array}$ & $\begin{array}{l}\text { BTN } \\
\text { Q7J }\end{array}$ & Smith & $09-17-91$ & $\begin{array}{l}732.7-751.0 \mathrm{~m} \\
(2404-2464 \mathrm{ft})\end{array}$ & $\begin{array}{l}18.3 \mathrm{~m} \\
(60 \mathrm{ft})\end{array}$ & 12 & Siltstone & \\
\hline $\begin{array}{l}\text { core } \\
\# 3\end{array}$ & $\begin{array}{l}22.2 \mathrm{~cm} \\
(8-3 / 4 \mathrm{in})\end{array}$ & $\begin{array}{l}\text { Marine core } \\
\text { 4-cone }\end{array}$ & NA & $09-23-91$ & $\begin{array}{l}751.0-757.7 \mathrm{~m} \\
(2464-2486 \mathrm{ft})\end{array}$ & $\begin{array}{l}6.7 \mathrm{~m} \\
(22 \mathrm{ft})\end{array}$ & NA & $\begin{array}{l}\text { Argillaceous } \\
\text { quartzite }\end{array}$ & $\begin{array}{l}\text { Recov. } 10 \mathrm{ft} \\
\text { lost lower } 12 \\
\mathrm{ft} \text { of core }\end{array}$ \\
\hline 14 & $\begin{array}{l}21.6 \mathrm{~cm} \\
(8-1 / 2 \mathrm{in})\end{array}$ & $\begin{array}{l}\text { BTN } \\
\text { AZ016 }\end{array}$ & $\begin{array}{l}\text { RBI } \\
\text { "Reed" }\end{array}$ & $09-23-91$ & $\begin{array}{l}751.0-757.7 \mathrm{~m} \\
(2464-2486 \mathrm{ft})\end{array}$ & $\begin{array}{l}6.7 \mathrm{~m} \\
(22 \mathrm{ft})\end{array}$ & NA & $\begin{array}{l}\text { Argillaceous } \\
\text { siltstone }\end{array}$ & \\
\hline 15 & $\begin{array}{l}31.1 \mathrm{~cm} \\
(12-1 / 4 \mathrm{in})\end{array}$ & $\begin{array}{l}\text { BTN } \\
\text { Q55 }\end{array}$ & Smith & $09-24-91$ & $\begin{array}{l}751.0-757.7 \mathrm{~m} \\
(2464-2486 \mathrm{ft})\end{array}$ & $\begin{array}{l}6.7 \mathrm{~m} \\
(22 \mathrm{ft})\end{array}$ & NA & $\begin{array}{l}\text { Argillaceous } \\
\text { quartzite }\end{array}$ & $\begin{array}{l}\text { Bit rerun on } \\
09-26-91\end{array}$ \\
\hline 16 & $\begin{array}{l}31.1 \mathrm{~cm} \\
(12-1 / 4 \mathrm{in})\end{array}$ & $\begin{array}{l}\text { MT } \\
\text { MXWR }\end{array}$ & Hughes & $09-25-91$ & $\begin{array}{l}757.7-771.8 \mathrm{~m} \\
(2486-2532 \mathrm{ft})\end{array}$ & $\begin{array}{l}14.0 \mathrm{~m} \\
(46 \mathrm{ft})\end{array}$ & 13.5 & $\begin{array}{l}\text { Siltstone \& } \\
\text { shale }\end{array}$ & \\
\hline 15 & $\begin{array}{l}31.1 \mathrm{~cm} \\
(12-1 / 4 \mathrm{in})\end{array}$ & $\begin{array}{l}\text { BTN } \\
\text { Q55 }\end{array}$ & Smith & $09-26-91$ & $\begin{array}{l}771.8-795.2 \mathrm{~m} \\
(2532-2609 \mathrm{ft})\end{array}$ & $\begin{array}{l}23.5 \mathrm{~m} \\
(77 \mathrm{ft})\end{array}$ & $\mathrm{NA}$ & $\begin{array}{l}\text { Shale \& } \\
\text { siltstone }\end{array}$ & \\
\hline 17 & $\begin{array}{l}31.1 \mathrm{~cm} \\
(12-1 / 4 \mathrm{in})\end{array}$ & $\begin{array}{l}\text { BTN } \\
05 \mathrm{~J}\end{array}$ & Smith & $10-01-91$ & $\begin{array}{l}795.2-846.1 \mathrm{~m} \\
(2609-2776 \mathrm{ft})\end{array}$ & $\begin{array}{l}50.9 \mathrm{~m} \\
(167 \mathrm{ft})\end{array}$ & NA & $\begin{array}{l}\text { Shale, } \\
\text { siltstone, \& } \\
\text { quartzite }\end{array}$ & $\begin{array}{l}T-8, B-8, \text { under } \\
\text { gauge }\end{array}$ \\
\hline 18 & $\begin{array}{l}31.1 \mathrm{~cm} \\
(12-1 / 4 \mathrm{in})\end{array}$ & $05 \mathrm{~J}$ & Smith & $10-03-91$ & $\begin{array}{l}846.1-885.7 \mathrm{~m} \\
(2776-2906 \mathrm{ft})\end{array}$ & $\begin{array}{l}39.6 \mathrm{~m} \\
(130 \mathrm{ft})\end{array}$ & NA & $\begin{array}{l}\text { Siltstone \& } \\
\text { dolomite }\end{array}$ & $\mathrm{T}-2, \mathrm{~B}-8, \mathrm{G}-2$ \\
\hline 19 & $\begin{array}{l}31.1 \mathrm{~cm} \\
(12-1 / 4 \mathrm{in})\end{array}$ & $\begin{array}{l}\text { BTN } \\
05 \mathrm{~J}\end{array}$ & Smith & $10-04-91$ & $\begin{array}{l}855.7-927.5 \mathrm{~m} \\
(2906-3043 \mathrm{ft})\end{array}$ & $\begin{array}{l}41.8 \mathrm{~m} \\
(137 \mathrm{ft})\end{array}$ & 20 & Dolomite & $\mathrm{T}-1 \mathrm{~B}-8$ in \\
\hline $\begin{array}{l}\text { core } \\
\# 4\end{array}$ & $\begin{array}{l}22.2 \mathrm{~cm} \\
(8-3 / 4 \mathrm{in})\end{array}$ & $\begin{array}{l}\text { Conventional } \\
\text { diamond core }\end{array}$ & Christensen & $10-07-91$ & $\begin{array}{l}895.2-903.1 \mathrm{~m} \\
(2937-2963 \mathrm{ft})\end{array}$ & $\begin{array}{l}7.9 \mathrm{~m} \\
(26 \mathrm{ft})\end{array}$ & NA & Dolomite & $\begin{array}{l}\text { Recovered } \\
7.8 \mathrm{~m}(25.5 \mathrm{ft}) \\
\text { from } 7.9 \mathrm{~m} \\
(26 \mathrm{ft}) \text { cut. }\end{array}$ \\
\hline 20 & $\begin{array}{l}31.1 \mathrm{~cm} \\
(12-1 / 4 \mathrm{in})\end{array}$ & BTN/05J & Smith & $10-09-91$ & $\begin{array}{l}927.5-941.5 \mathrm{~m} \\
(3043-3089 \mathrm{ft})\end{array}$ & $\begin{array}{l}14.0 \mathrm{~m} \\
(46 \mathrm{ft})\end{array}$ & 5.5 & Dolomite & $\begin{array}{l}\mathrm{T}-1, \mathrm{~B}-2, \text { in } \\
\text { gauge }\end{array}$ \\
\hline
\end{tabular}


Table 3. Bit Records for ER-12-1 (continued).

\begin{tabular}{|c|c|c|c|c|c|c|c|c|c|}
\hline $\begin{array}{l}\text { Bit } \\
\text { No. }\end{array}$ & $\begin{array}{l}\text { Bit } \\
\text { Diameter }\end{array}$ & $\begin{array}{l}\text { Bit } \\
\text { Type }\end{array}$ & Manufacturer & Date In & $\begin{array}{l}\text { Depth Interval } \\
\text { Drilled }\end{array}$ & $\begin{array}{l}\text { Footage } \\
\text { Drilled }\end{array}$ & $\begin{array}{l}\text { Hours } \\
\text { Drilled }\end{array}$ & $\begin{array}{l}\text { Lithology(s) } \\
\text { Drilled }\end{array}$ & $\begin{array}{l}\text { Comments/ } \\
\text { Bit Grade }\end{array}$ \\
\hline 21 & $\begin{array}{l}31.1 \mathrm{~cm} \\
(12-1 / 4 \mathrm{in})\end{array}$ & $\begin{array}{l}\text { BTN } \\
\text { BH-80 }\end{array}$ & Hughes & $10-10-91$ & $\begin{array}{l}941.5-967.4 \mathrm{~m} \\
(3089-3174 \mathrm{ft})\end{array}$ & $\begin{array}{l}25.9 \mathrm{~m} \\
(85 \mathrm{ft})\end{array}$ & 31.5 & Dolomite & $\begin{array}{l}T-3, B-2, \text { in } \\
\text { gauge }\end{array}$ \\
\hline 22 & $\begin{array}{l}31.1 \mathrm{~cm} \\
(12-1 / 4 \mathrm{in})\end{array}$ & $\begin{array}{l}\text { BTN } \\
\text { BH-80 }\end{array}$ & Hughes & $10-11-91$ & $\begin{array}{l}967.4-969.6 \mathrm{~m} \\
(3174-3181 \mathrm{ft})\end{array}$ & $\begin{array}{l}2.1 \mathrm{~m} \\
(7 \mathrm{ft})\end{array}$ & 4 & Dolomite & $\begin{array}{l}T-1, B-4 \text {, in } \\
\text { gauge }\end{array}$ \\
\hline 23 & $\begin{array}{l}31.1 \mathrm{~cm} \\
(12-1 / 4 \text { in })\end{array}$ & $\begin{array}{l}\text { BTN } \\
05 \mathrm{~J}\end{array}$ & Smith & $10-14-91$ & $\begin{array}{l}969.6-1018.6 \mathrm{~m} \\
(3183-3342 \mathrm{ft})\end{array}$ & $\begin{array}{l}49.0 \mathrm{~m} \\
(161 \mathrm{ft})\end{array}$ & 21 & Dolomite & $\begin{array}{l}T-1, B-4, \text { in } \\
\text { gauge }\end{array}$ \\
\hline 24 & $\begin{array}{l}31.1 \mathrm{~cm} \\
(12-1 / 4 \mathrm{in})\end{array}$ & $\begin{array}{l}\text { BTN } \\
05 \mathrm{~J}\end{array}$ & Smith & $10-15-91$ & $\begin{array}{l}1018.6-1092.4 \mathrm{~m} \\
(3342-3588 \mathrm{ft})\end{array}$ & $\begin{array}{l}75.0 \mathrm{~m} \\
(246 \mathrm{ft})\end{array}$ & 34.5 & Dolomite* & $\begin{array}{l}\text { T-6, B-8, in } \\
\text { gauge }\end{array}$ \\
\hline
\end{tabular}

\section{Bit Statistics}

$\begin{array}{lll}\text { No. of Bits } & \text { Bit Diameter } & \text { Avg. Drilled } \\ & & \\ 2 \text { bits } & \begin{array}{l}4.0 \mathrm{~cm} \\ (18-1 / 2 \mathrm{in})\end{array} & \begin{array}{l}24.7 \mathrm{~m} \\ (81 \mathrm{ft})\end{array} \\ & & \\ 3 \mathrm{bits} & 44.5 \mathrm{~cm} & 227.7 \mathrm{~m} \\ & (17-1 / 2 \mathrm{in}) & (747 \mathrm{ft}) \\ & & \\ 18 \text { bits } & 31.1 \mathrm{~cm} & 367 \mathrm{~m} \\ & (12-1 / 4 \mathrm{in}) & (120.4 \mathrm{ft})\end{array}$

\section{Hours Drilled/Bit}

$45 \mathrm{hrs} / \mathrm{bit}$ (for the 2 bits with data)

$41.5 \mathrm{hrs} / \mathrm{bit}$ (for the 2 bits with data)

$21.4 \mathrm{hrs} / \mathrm{bit}$ (for the 11 bits with data)

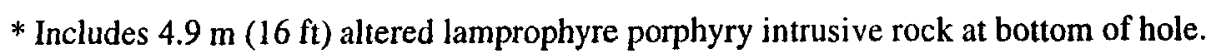
NA - Not readily available from REECo drilling records. 
Table 4. Hole Deviation Survey Records for ER-12-1.

\begin{tabular}{|c|c|c|c|c|}
\hline Survey Depth & $\begin{array}{l}\text { Deviation } \\
\text { REECo }\end{array}$ & $\begin{array}{c}\text { Deviation } \\
\text { Schlumberger }\end{array}$ & $\begin{array}{c}\text { Survey Date } \\
\text { REECo }\end{array}$ & $\begin{array}{l}\text { Survey Date } \\
\text { Schlumberger }\end{array}$ \\
\hline $28.3 \mathrm{~m}(93 \mathrm{ft})$ & $* 1^{\circ} 16^{\prime}$ reported & N/A & $07-30-91$ & NA \\
\hline $35.7 \mathrm{~m}(117 \mathrm{ft})$ & ${ }^{*}$ off scale using a $2^{\circ}$ tool & N/A & $07-31-91$ & NA \\
\hline $53.3 \mathrm{~m}(175 \mathrm{ft})$ & $* 3^{\circ} 14^{\prime}$ reported & N/A & $07-31-91$ & NA \\
\hline $26.2 \mathrm{~m}(86 \mathrm{ft})$ & $* 1^{\circ} 11^{\prime}$ reported & N/A & $08-07-91$ & NA \\
\hline $32.6 \mathrm{~m}(107 \mathrm{ft})$ & $* 1^{\circ} 16^{\prime}$ reported & N/A & $08-07-91$ & NA \\
\hline $51.5 \mathrm{~m}(169 \mathrm{ft})$ & $* 3^{\circ} 48^{\prime}$ reported & N/A & $08-07-91$ & NA \\
\hline $44.2 \mathrm{~m}(145 \mathrm{ft})$ & $0^{\circ} 25^{\prime}$ & N/A & $08-08-91$ & NA \\
\hline $62.2 \mathrm{~m}(204 \mathrm{ft})$ & $0^{\circ} 17^{\prime}$ & $\mathrm{N} / \mathrm{A}$ & $08-08-91$ & NA \\
\hline $101.2 \mathrm{~m}(332 \mathrm{ft})$ & $0^{\circ} 18^{\prime}$ & N/A & $08-09-91$ & NA \\
\hline $130.1 \mathrm{~m}(427 \mathrm{ft})$ & $0^{\circ} 12^{\prime}$ & N/A & $08-09-91$ & NA \\
\hline $195.7 \mathrm{~m}(642 \mathrm{ft})$ & $0^{\circ} 36^{\prime}$ & N/A & $08-13-91$ & NA \\
\hline $301.1 \mathrm{~m}(988 \mathrm{ft})$ & $0^{\circ} 55^{\prime}$ & N/A & $08-14-91$ & NA \\
\hline $393.5 \mathrm{~m}(1291 \mathrm{ft})$ & $1^{\circ} 10^{\prime}$ & N/A & $08-16-91$ & NA \\
\hline $480.1 \mathrm{~m}(1575 \mathrm{ft})$ & $0^{\circ} 40^{\prime}$ & $1.35^{\circ}$ & $08-19-91$ & $10-22-91$ \\
\hline $515.1 \mathrm{~m}(1690 \mathrm{ft})$ & $1^{\circ} 5^{\prime}$ & $1.1^{\circ}$ & $08-20-91$ & $10-22-91$ \\
\hline $580.6 \mathrm{~m}(1905 \mathrm{ft})$ & $1^{\circ} 55^{\prime}$ & $1.8^{\circ}$ & $09-09-91$ & $10-22-91$ \\
\hline $680.9 \mathrm{~m}(2234 \mathrm{ft})$ & $1^{\circ} 12^{\prime}$ & $4.75^{\circ}$ & $09-12-91$ & $10-22-91$ \\
\hline $780.3 \mathrm{~m}(2560 \mathrm{ft})$ & $1^{\circ} 10^{\prime}$ & $6.4^{\circ}$ & $09-26-91$ & $10-22-91$ \\
\hline $831.2 \mathrm{~m}(2727 \mathrm{ft})$ & $1^{\circ} 18^{\prime}$ & $8.4^{\circ}$ & $10-03-91$ & $10-22-91$ \\
\hline $897.6 \mathrm{~m}(2945 \mathrm{ft})$ & $1^{\circ} 10^{\prime}$ & $7.2^{\circ}$ & $10-08-91$ & $10-22-91$ \\
\hline
\end{tabular}

* Readings invalid, improper use of tool (upside down) 
Schlumberger survey indicated an increase in hole deviation from approximately $2^{\circ}$ to almost $9.5^{\circ}$ at $865.6 \mathrm{~m}$ ( $2840 \mathrm{ft})$. Below $865.5 \mathrm{~m}$, hole deviation gradually decreased to slightly more than $3^{\circ}$ near total depth (TD) at $1092 \mathrm{~m}$ ( $3582 \mathrm{ft})$. The fairly consistent hole deviation values recorded by REECo during drilling appear to indicate there was a problem with the tool, which prevented the measurement of hole deviations greater than approximately $2^{\circ}$. The measurements by Schlumberger are, where run, a more accurate representation.

Fluids used in drilling ER-12-1, including dates, depth intervals, and quantities, are listed in Table 5. Bentonite drilling mud was used in ER-12-1 from approximately $548.6 \mathrm{~m}(1800 \mathrm{ft})$ to TD. The use of drilling mud was necessitated by loss of circulation and difficulty cleaning the hole due to the use of inadequate diameter (for reverse circulation) integral dual string drill pipe. Mud weights and viscosity measurements are listed in Table 6.

Additional problems encountered during construction of ER-12-1 included insufficient bit life, hammer drill foot valve failures, fishing operations for parted drill pipe and rotary bit cones, poor core recovery, stuck drill pipe, and, finally, deteriorating hole conditions after the removal of the bentonite drilling fluid during initial well development. Many of these problems, along with other important information about the drilling of ER-12-1, are listed in the excerpts from the "Driller's notes" in Table 7.

Following completion of drilling at ER-12-1, initial attempts were made to develop the well by circulating a sodium tetraphosphate solution in the hole to disperse the bentonite-based drilling mud used during construction. The types of development fluids used in ER-12-1, including dates and quantities, are presented in Table 8. Initial well development activities were abandoned due to excessive fill produced in the hole upon removal of the bentonite mud.

To remove the fill and restabilize the wellbore, the hole was cleaned and conditioned on Jan. 13, 1992, using first a bentonite-based drilling mud, and then a polymer mud. Once the wellbore stabilized on Jan. 24, 1992, 19.4- $\mathrm{cm}$ (7 5/8 in) steel casing with five slotted intervals was gravel packed and cemented within the hole (Figure 2) to stabilize the hole during further well development and initial hydrologic testing of the well. The five slotted intervals were placed opposite representative intervals of the formations encountered in the borehole. The representative intervals were chosen, at a meeting on October 31, 1991 (attended by RSN, DRI, and USGS), to represent various formations within the borehole and their associated permeability. The zones of interest, geologic formation, and rationale for completing these intervals are shown in Table 9. As illustrated in Figure 2, some difficulty was encountered in placing the cement within the well. Cement extends $8.5 \mathrm{~m}(28 \mathrm{ft})$ above the bottom of the slotted interval in the lowermost zone. At the middle and

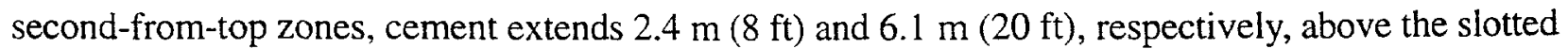
intervals. These errors in cement placement probably resulted from fill from the wellbore wall falling into the well during the cementing operation, resulting in an excessive rise in cement.

With the hole stabilized by the placement of the slotted casing, it was possible to place a submersible pump within the hole and complete an initial phase of well development. A record of the amounts of fluids removed from the hole, along with dates, methods, and comments is contained in Table 10. 
Table 5. Drilling Fluid Records for ER-12-1.

\begin{tabular}{|c|c|c|}
\hline Date & Approximate Depth & Volume, Fluid Type, Comments \\
\hline $07-18-91$ & 0 & $312 \mathrm{~m}^{3}$ (1962 bbls) of water to circulation pit \\
\hline $07-19-91$ & 0 & $286 \mathrm{~m}^{3}(1800 \mathrm{bbls})$ of water \\
\hline $07-22-91$ & $6.1 \mathrm{~m}(20 \mathrm{ft})$ & $\begin{array}{l}19 \mathrm{~m}^{3}(120 \mathrm{bbls}) \text { of } 50 \text { vis mud, } 129 \mathrm{~kg} \\
(286 \mathrm{lbs}) \text { of water }\end{array}$ \\
\hline $07-23-91$ & $6.1 \mathrm{~m}(20 \mathrm{ft})$ & $19 \mathrm{~m}^{3}(120 \mathrm{bbls})$ of 50 vis mud, no water \\
\hline $07-24-91$ & $6.1 \mathrm{~m}(20 \mathrm{ft})$ & $32 \mathrm{~m}^{3}$ (200 bbls) of water \\
\hline $07-25-91$ & $21.3 \mathrm{~m}(70 \mathrm{ft})$ & $32 \mathrm{~m}^{3}(200 \mathrm{bbls})$ of water \\
\hline $07-30-91$ & $39.6 \mathrm{~m}(130 \mathrm{ft})$ & $68 \mathrm{~m}^{3}$ (429 bbls) of water \\
\hline $07-31-91$ & $53.3 \mathrm{~m}(175 \mathrm{ft})$ & $222 \mathrm{~m}^{3}(1400 \mathrm{bbls})$ of water \\
\hline $08-01-91$ & $64.0 \mathrm{~m}(210 \mathrm{ft})$ & $127 \mathrm{~m}^{3}(800 \mathrm{bbls})$ of water \\
\hline $08-05-91$ & $64.0 \mathrm{~m}(210 \mathrm{ft})$ & $\begin{array}{l}38 \mathrm{~m}^{3}(240 \mathrm{bbls}) \text { of Davis Mix, } 45.3 \mathrm{~kg} \\
(100 \mathrm{lbs}) \text { of water }\end{array}$ \\
\hline $08-07-91$ & $64.0 \mathrm{~m}(210 \mathrm{ft})$ & $57 \mathrm{~m}^{3}$ (360 bbls) of Davis Mix \\
\hline $08-08-91$ & $64.0 \mathrm{~m}(210 \mathrm{ft})$ & $38 \mathrm{~m}^{3}$ (240 bbls) of Davis Mix \\
\hline $08-09-91$ & $121.9 \mathrm{~m}(400 \mathrm{ft})$ & $114 \mathrm{~m}^{3}(720 \mathrm{bbls})$ of Davis Mix \\
\hline $08-12-91$ & $152.4 \mathrm{~m}(500 \mathrm{ft})$ & $57 \mathrm{~m}^{3}$ (360 bbls) of Davis Mix \\
\hline $08-13-91$ & $213.4 \mathrm{~m}(700 \mathrm{ft})$ & $96 \mathrm{~m}^{3}$ (600 bbls) of Davis Mix \\
\hline $08-14-91$ & $304.8 \mathrm{~m}(1000 \mathrm{ft})$ & $76 \mathrm{~m}^{3}$ (480 bbls) of Davis Mix \\
\hline $08-15-91$ & $365.8 \mathrm{~m}(1200 \mathrm{ft})$ & $38 \mathrm{~m}^{3}(240 \mathrm{bbls})$ of Davis Mix \\
\hline $08-16-91$ & $411.5 \mathrm{~m}(1350 \mathrm{ft})$ & $38 \mathrm{~m}^{3}$ (240 bbls) of Davis Mix \\
\hline $08-19-91$ & $487.7 \mathrm{~m}(1600 \mathrm{ft})$ & $114 \mathrm{~m}^{3}(720 \mathrm{bbls})$ of Davis Mix \\
\hline $08-27-91$ & $457.2 \mathrm{~m}(1500 \mathrm{ft})$ & $\begin{array}{l}153 \mathrm{~m}^{3} \text { ( } 960 \text { bbls) of water; drilling sand } \\
\& \text { cement from casing }\end{array}$ \\
\hline $08-28-91$ & $457.2 \mathrm{~m}(1500 \mathrm{ft})$ & $\begin{array}{l}96 \mathrm{~m}^{3}(600 \text { bbls }) \text { of water; drilling sand } \\
\& \text { cement from casing }\end{array}$ \\
\hline $08-29-91$ & $518.2 \mathrm{~m}(1700 \mathrm{ft})$ & $\begin{array}{l}67 \mathrm{~m}^{3} \text { ( } 420 \text { bbls) of Davis Mix; drilling } \\
\text { formation }\end{array}$ \\
\hline $09-03-91$ & $548.6 \mathrm{~m}(1800 \mathrm{ft})$ & $76 \mathrm{~m}^{3}(480 \mathrm{bbls})$ of Davis Mix \\
\hline 09-04-91 & $548.6 \mathrm{~m}(1800 \mathrm{ft})$ & $19 \mathrm{~m}^{3}(120 \mathrm{bbls})$ of Davis Mix \\
\hline $09-05-91$ & $548.6 \mathrm{~m}(1800 \mathrm{ft})$ & $\begin{array}{l}153 \mathrm{~m}^{3}(960 \mathrm{bbls}) \text { of water; } 490 \mathrm{~kg} \\
(1080 \mathrm{lbs}) \text { of } 60 \mathrm{vis} \text { mud }\end{array}$ \\
\hline $09-06-91$ & $548.6 \mathrm{~m}(1800 \mathrm{ft})$ & $306 \mathrm{~m}^{3}(1920 \mathrm{bbls})$ of mud \\
\hline $09-09-91$ & $579.1 \mathrm{~m}(1900 \mathrm{ft})$ & $172 \mathrm{~m}^{3}(1080 \mathrm{bbls})$ of mud \\
\hline $09-10-91$ & $609.6 \mathrm{~m}(2000 \mathrm{ft})$ & $172 \mathrm{~m}^{3}(1080 \mathrm{bbls})$ of mud \\
\hline $09-11-91$ & $640.1 \mathrm{~m}(2100 \mathrm{ft})$ & $19 \mathrm{~m}^{3}(120 \mathrm{bbls})$ of 60 vis mud \\
\hline $09-12-91$ & $670.6 \mathrm{~m}(2200 \mathrm{ft})$ & $76 \mathrm{~m}^{3}$ ( $\left.480 \mathrm{bbls}\right)$ of 60 vis mud \\
\hline
\end{tabular}


Table 5. Drilling Fluid Records for ER-12-1 (continued).

\begin{tabular}{|c|c|c|}
\hline Date & Approximate Depth & Volume, Fluid Type, Comments \\
\hline $09-16-91$ & $701.0 \mathrm{~m}(2300 \mathrm{ft})$ & $150 \mathrm{~m}^{3}(940 \mathrm{bbls})$ of $60 \mathrm{vis}$ mud \\
\hline $09-17-91$ & $731.5 \mathrm{~m}(2400 \mathrm{ft})$ & $198 \mathrm{~m}^{3}(120 \mathrm{bbls})$ of mud \\
\hline $09-18-91$ & $731.5 \mathrm{~m}(2400 \mathrm{ft})$ & $57 \mathrm{~m}^{3}(360 \mathrm{bbls})$ of mud \\
\hline $09-23-91$ & $731.5 \mathrm{~m}(2400 \mathrm{ft})$ & $\begin{array}{l}19 \mathrm{~m}^{3}(120 \mathrm{bbls}) \text { of water; } 54 \mathrm{~kg} \\
(120 \mathrm{lbs}) \text { of mud }\end{array}$ \\
\hline $09-25-91$ & $731.5 \mathrm{~m}(2400 \mathrm{ft})$ & $76 \mathrm{~m}^{3}(480 \mathrm{bbls})$ of mud \\
\hline $09-26-91$ & $762.0 \mathrm{~m}(2500 \mathrm{ft})$ & $19 \mathrm{~m}^{3}(120 \mathrm{bbls})$ of 60 vis mud \\
\hline $10-02-91$ & $792.5 \mathrm{~m}(2600 \mathrm{ft})$ & $76 \mathrm{~m}^{3}(480 \mathrm{bbls})$ of mud \\
\hline $10-03-91$ & $823.0 \mathrm{~m}(2700 \mathrm{ft})$ & $114 \mathrm{~m}^{3}(720 \mathrm{bbls})$ of mud \\
\hline $10-04-91$ & $853.4 \mathrm{~m}(2800 \mathrm{ft})$ & $76 \mathrm{~m}^{3}(480 \mathrm{bbls})$ of mud \\
\hline $10-07-91$ & $883.9 \mathrm{~m}(2900 \mathrm{ft})$ & $38 \mathrm{~m}^{3}(240 \mathrm{bbls})$ of mud \\
\hline $10-08-91$ & $883.9 \mathrm{~m}(2900 \mathrm{ft})$ & $38 \mathrm{~m}^{3}(240 \mathrm{bbls})$ of mud \\
\hline $10-09-91$ & $914.4 \mathrm{~m}(3000 \mathrm{ft})$ & $134 \mathrm{~m}^{3}(840 \mathrm{bbls})$ of mud \\
\hline $10-10-91$ & $944.9 \mathrm{~m}(3100 \mathrm{ft})$ & $538 \mathrm{~m}^{3}(3380 \mathrm{bbls})$ of mud; lost circulation \\
\hline $10-11-91$ & $944.9 \mathrm{~m}(3100 \mathrm{ft})$ & $\begin{array}{l}395 \mathrm{~m}^{3}(2480 \mathrm{bbls}) \text { of } 60 \text { vis mud; } 109 \mathrm{~kg} \\
(240 \mathrm{lbs}) \text { of } 120 \text { vis mud }\end{array}$ \\
\hline $10-14-91$ & $975.4 \mathrm{~m}(3200 \mathrm{ft})$ & $251 \mathrm{~m}^{3}(1580 \mathrm{bbls})$ of mud \\
\hline $10-15-91$ & $1005.8 \mathrm{~m}(3300 \mathrm{ft})$ & $191 \mathrm{~m}^{3}(1200 \mathrm{bbls})$ of mud \\
\hline $10-16-91$ & $1036.3 \mathrm{~m} \mathrm{(3400 \textrm {ft } )}$ & $134 \mathrm{~m}^{3}(840 \mathrm{bbls})$ of mud \\
\hline $10-17-91$ & $1066.8 \mathrm{~m}(3500 \mathrm{ft})$ & $57 \mathrm{~m}^{3}(360 \mathrm{bbls})$ of mud; ER-12-1 reaches TD \\
\hline $10-23-91$ & $1093.6 \mathrm{~m}(3588 \mathrm{ft})$ & $777 \mathrm{~m}^{3}(4880 \mathrm{bbls})$ of water \\
\hline $10-24-91$ & $1093.6 \mathrm{~m}(3588 \mathrm{ft})$ & $595 \mathrm{~m}^{3}(3740 \mathrm{bbls})$ of water \\
\hline
\end{tabular}

Miscellaneous Fluids

Date Approximate Depth Additive/Comments

08-14-91

$08-15-91$
$354.2 \mathrm{~m}(1162 \mathrm{ft})$

$354.2 \mathrm{~m}(1162 \mathrm{ft})$
While out of hole, put 2 gallons of oil down drill collars to lubricate bit

Rigged up circulating system to blend in lithium bromide tracer; used 1 gallon lithium bromide per $79 \mathrm{~m}^{3}$ (500 bbls) of water. 
Table 6. Drilling Mud Data for ER-12-1.

\begin{tabular}{|c|c|c|c|c|c|}
\hline Date & Depth & Vis In & Vis Out & Weight & Comments \\
\hline $09-06-91$ & $\begin{array}{r}556.9 \mathrm{~m} \\
(1827 \mathrm{ft})\end{array}$ & $\mathrm{NA}$ & NA & $\mathrm{NA}$ & $\begin{array}{l}\text { Pumped } 169 \mathrm{~m}^{3}(1060 \mathrm{bbls}) \\
\text { of mud down hole with } \\
\text { no returns }\end{array}$ \\
\hline 09-09-91 & $\begin{array}{r}617.8 \mathrm{~m} \\
(2027 \mathrm{ft})\end{array}$ & 68 & 78 & NA & Drilling with partial returns \\
\hline $09-12-91$ & $\begin{array}{r}693.7 \mathrm{~m} \\
(2276 \mathrm{ft})\end{array}$ & 60 & NA & NA & Drilling with full returns \\
\hline $09-18-91$ & $\begin{array}{r}751.0 \mathrm{~m} \\
(2464 \mathrm{ft})\end{array}$ & 70 & NA & NA & \\
\hline $10-01-91$ & $\begin{array}{r}798.9 \mathrm{~m} \\
(2621 \mathrm{ft})\end{array}$ & 56 & 56 & 8.5 & \\
\hline $10-03-91$ & $\begin{array}{r}876.6 \mathrm{~m} \\
(2876 \mathrm{ft})\end{array}$ & 52 & 52 & 8.7 & \\
\hline $10-04-91$ & $\begin{array}{r}885.7 \mathrm{~m} \\
(2906 \mathrm{ft})\end{array}$ & 56 & 56 & 8.7 & \\
\hline $10-07-91$ & $\begin{array}{r}895.2 \mathrm{~m} \\
(2937 \mathrm{ft})\end{array}$ & 49 & 52 & 8.6 & \\
\hline $10-08-91$ & $\begin{array}{r}927.5 \mathrm{~m} \\
(3043 \mathrm{ft})\end{array}$ & 44 & 44 & $\begin{array}{l}8.9 \mathrm{in} / \\
8.6 \text { out }\end{array}$ & \\
\hline $10-09-91$ & $\begin{array}{r}942.7 \mathrm{~m} \\
(3093 \mathrm{ft})\end{array}$ & 50 & 47 & 8.7 & $\begin{array}{l}\text { Fluid loss at drilling break } \\
\text { at } 935.1 \mathrm{~m}(3068 \mathrm{ft})\end{array}$ \\
\hline $10-10-91$ & $\begin{array}{r}958.0 \mathrm{~m} \\
(3143 \mathrm{ft})\end{array}$ & 60 & $\mathrm{NA}$ & 8.5 & $\begin{array}{l}\text { Received } 538 \mathrm{~m}^{3} \text { (3380 bbls) } \\
\text { of mud }\end{array}$ \\
\hline $10-11-91$ & $\begin{array}{r}967.4 \mathrm{~m} \\
(3174 \mathrm{ft})\end{array}$ & 120 & 144 & $\begin{array}{l}8.7 \mathrm{in} / \\
8.8 \text { out }\end{array}$ & $\begin{array}{l}\text { Drill bit hit void from } \\
962.9-963.8 \mathrm{~m}(3159-3162 \\
\mathrm{ft}) ; \text { received } 280 \mathrm{~m}^{3} \\
(1760 \text { bbls }) \text { of mud }\end{array}$ \\
\hline $10-14-91$ & $\begin{array}{r}968.7 \mathrm{~m} \\
(3178 \mathrm{ft})\end{array}$ & 110 & NA & 8.7 & $\begin{array}{l}\text { Dirty-black water being } \\
\text { displaced out of hole }\end{array}$ \\
\hline $10-14-91$ & $\begin{array}{r}991.5 \mathrm{~m} \\
(3253 \mathrm{ft})\end{array}$ & 75 & NA & 8.7 & \\
\hline $10-15-91$ & $\begin{array}{l}1030.5 \mathrm{~m} \\
(3381 \mathrm{ft})\end{array}$ & 58 & 69 & $\begin{array}{l}8.7 \mathrm{in} / \\
8.9 \text { out }\end{array}$ & $\begin{array}{l}\text { Received } 172 \mathrm{~m}^{3}(1080 \mathrm{bbls}) \\
\text { of } 80 \text { vis mud }\end{array}$ \\
\hline $10-16-91$ & $\begin{array}{l}1066.8 \mathrm{~m} \\
(3500 \mathrm{ft})\end{array}$ & 90 & 68 & $\begin{array}{l}8.4 \mathrm{in} / \\
8.7 \text { out }\end{array}$ & \\
\hline $10-17-91$ & $\begin{array}{l}1093.6 \mathrm{~m} \\
(3588 \mathrm{ft})\end{array}$ & 75 & 75 & $\begin{array}{l}9.0 \mathrm{in} / \\
8.8 \text { out }\end{array}$ & Mud check taken at $0920 \mathrm{hrs}$ \\
\hline $10-17-91$ & $\begin{array}{l}1093.6 \mathrm{~m} \\
(3588 \mathrm{ft})\end{array}$ & 47 & 57 & $\begin{array}{l}8.8 \text { in } / \\
8.9 \text { out }\end{array}$ & $\begin{array}{l}\text { Mud check taken at } \\
1400 \text { hrs; ER-12-1 TD'd }\end{array}$ \\
\hline
\end{tabular}


Table 7. Driller's Notes.

\begin{tabular}{|c|c|c|}
\hline Date & Depth & Comments \\
\hline $07-19-91$ & $3.7 \mathrm{~m}(12 \mathrm{ft})$ & Spud date \\
\hline $07-22-91$ & $9.1 \mathrm{~m}(30 \mathrm{ft})$ & Hole not straight \\
\hline $07-25-91$ & $22.6 \mathrm{~m}(74 \mathrm{ft})$ & Geolograph calibrated and deficiency noted \\
\hline $07-26-91$ & $30.5 \mathrm{~m}(100 \mathrm{ft})$ & $\begin{array}{l}\text { Problem cleaning hole so change circulation systems to reverse } \\
\text { air/water }\end{array}$ \\
\hline $07-29-91$ & $30.5 \mathrm{~m}(100 \mathrm{ft})$ & $3.7 \mathrm{~m}(12 \mathrm{ft})$ of fill after weekend; tight hole, drive line snapped \\
\hline $08-01-91$ & $65.5 \mathrm{~m}(215 \mathrm{ft})$ & Receive orders at $1700 \mathrm{hrs}$ to plug hole \\
\hline $08-07-91$ & $58.2 \mathrm{~m}(191 \mathrm{ft})$ & $\begin{array}{l}\text { Hammer drill (bit \#6) not working properly, pin from float had } \\
\text { jammed; survey tool problem corrected, tool had been run upside } \\
\text { down }\end{array}$ \\
\hline $08-12-91$ & $179.8 \mathrm{~m}(590 \mathrm{ft})$ & $\begin{array}{l}\text { Foot valve broke in hammer drill (bit \#7); button bit is used but } \\
\text { won't go past } 18.3 \mathrm{~m}(60 \mathrm{ft}) \text { depth so ream hole from } 18.3-24.4 \\
\mathrm{~m}(60 \text { to } 80 \mathrm{ft}) \text {; stabilizer drags } 2.2-4.5 \mathrm{~K} \mathrm{~kg}(5-10 \mathrm{~K} \text { lbs) when } \\
\text { going through bottom of surface casing }\end{array}$ \\
\hline 08-13-91 & $238.7 \mathrm{~m}(783 \mathrm{ft})$ & Bit \#7 and bottom reamers are $.64 \mathrm{~cm}$ (1/4 in) under gauge \\
\hline $08-15-91$ & $370.9 \mathrm{~m}(1217 \mathrm{ft})$ & No fill after event (rig secured $<24 \mathrm{hrs)}$ \\
\hline $08-16-91$ & $423.7 \mathrm{~m}(1390 \mathrm{ft})$ & Replace foot valve on hammer drill (bit \#7) \\
\hline $08-30-91$ & $539.2 \mathrm{~m}(1769 \mathrm{ft})$ & $\begin{array}{l}\text { Cored } 4.6 \mathrm{~m}(15 \mathrm{ft}) \text { recovered } 0.06 \mathrm{~m}(0.2 \mathrm{ft}) \text { from core } \# 1 \text { using } \\
\text { the conventional core barrel }\end{array}$ \\
\hline $09-03-91$ & $556.9 \mathrm{~m}(1827 \mathrm{ft})$ & $\begin{array}{l}\text { Cored } 8.2 \mathrm{~m}(27 \mathrm{ft}) \text {, recovered } 0.0 \mathrm{~m}(0 \mathrm{ft}) \text { from core run } \# 2 \text {; ream } \\
\& \text { clean hole to } 656.9 \mathrm{~m}(1827 \mathrm{ft}) \text {, then pipe gets stuck }\end{array}$ \\
\hline 09-04-91 & $546.8 \mathrm{~m}(1794 \mathrm{ft})$ & 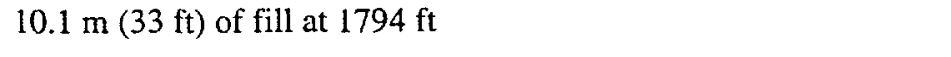 \\
\hline $09-05-91$ & $\begin{array}{l}544.1-556.9 \mathrm{~m} \\
(1785-1827 \mathrm{ft})\end{array}$ & Reverse circulation not cleaning hole, pipe gets stuck \\
\hline $09-06-91$ & $556.9 \mathrm{~m}(1827 \mathrm{ft})$ & $\begin{array}{l}\text { Added } 169 \mathrm{~m}^{3}(1060 \text { bbls }) \text { of mud with no returns (total hole } \\
\text { volume was approximately } 53 \mathrm{~m}^{3}(333 \text { bbls)) }\end{array}$ \\
\hline $9-11-91$ & $653.8 \mathrm{~m}(2145 \mathrm{ft})$ & $\begin{array}{l}\text { Trip in hole with new bit (\#11); encountered } 4.9 \mathrm{~m} \text { (16 ft) of fill } \\
\text { after event (rig secured }<24 \mathrm{hrs)}\end{array}$ \\
\hline $09-16-91$ & $693.7 \mathrm{~m}(2276 \mathrm{ft})$ & No fill after event on weekend (rig secured for 3 days) \\
\hline $09-18-91$ & $737.0 \mathrm{~m}(2418 \mathrm{ft})$ & $\begin{array}{l}\text { Ream tight hole from } 548.3-556.9 \mathrm{~m}(1799-1827 \mathrm{ft}) \text { and from } \\
723.9-732.7 \mathrm{~m}(2375-2404 \mathrm{ft}) \text { after trip for new bit }\end{array}$ \\
\hline $09-23-91$ & $751.0 \mathrm{~m}(2464 \mathrm{ft})$ & $\begin{array}{l}\text { Swap out duplex mud pump for triplex mud pump and pick up } \\
\text { core barrel for core } \# 3,751.0-757.7 \mathrm{~m}(2464-2486 \mathrm{ft})\end{array}$ \\
\hline
\end{tabular}


Table 7. Driller's Notes (continued).

\begin{tabular}{|c|c|c|}
\hline Date & Depth & Comments \\
\hline 09-25-91 & $757.7 \mathrm{~m}(2486 \mathrm{ft})$ & $\begin{array}{l}\text { Measured drill pipe while tripping in hole-no depth correction; } \\
\text { left button rotary cones from marine core bit in hole; retrieved } \\
\text { using mill tooth bit and globe basket }\end{array}$ \\
\hline $09-27-91$ & $795.2 \mathrm{~m}(2609 \mathrm{ft})$ & $\begin{array}{l}\text { Drill pipe twisted off after connection at } 792.8 \mathrm{~m}(2601 \mathrm{ft}) \text {; pipe } \\
\text { broke at weld } 7.62 \mathrm{~cm}(3 \text { in) above pipe collar; successfully } \\
\text { retrieved fish in four trips }\end{array}$ \\
\hline $10-02-91$ & $795.2 \mathrm{~m}(2609 \mathrm{ft})$ & $\begin{array}{l}\text { Laid down } 13.97 \mathrm{~cm}(5-1 / 2 \text { in) dual string drill pipe and picked } \\
\text { up } 16.83 \mathrm{~cm}(6-5 / 8 \mathrm{in}) \text { full-hole drill pipe; began drilling } 31.12 \\
\mathrm{~cm}(12-1 / 4 \mathrm{in}) \text { hole with } 19.69 \mathrm{~cm}(7-3 / 4 \text { in) Dynadrill }\end{array}$ \\
\hline $10-03-91$ & $846.1 \mathrm{~m}(2776 \mathrm{ft})$ & $21.3 \mathrm{~m}$ (70 ft) of fill after trip for new bit \\
\hline 10-03-91 & $853.4 \mathrm{~m}(2800 \mathrm{ft})$ & Changed out mud pumps to duplex \\
\hline 10-08-91 & $\begin{array}{l}895.2-903.1 \mathrm{~m} \\
(2937-2963 \mathrm{ft})\end{array}$ & $\begin{array}{l}\text { Recovered } 7.8 \mathrm{~m}(25.5 \mathrm{ft}) \text { from } 7.9 \mathrm{~m}(26 \mathrm{ft}) \text { cut on core } \\
\text { run \#4; change out mud pumps }\end{array}$ \\
\hline $10-09-91$ & $\begin{array}{l}833.6-935.1 \mathrm{~m} \\
(2735-3068 \mathrm{ft})\end{array}$ & $\begin{array}{l}\text { Ream tight hole from } 833.6-860.5 \mathrm{~m} \text { ( } 2735 \\
\text { to } 2823 \mathrm{ft}) ; \text { drilling break and fluid loss at } 935.1 \mathrm{~m} \\
(3068 \mathrm{ft}) \text {; drilled } 1.5 \mathrm{~m}(5 \mathrm{ft}) \text { in } 8 \text { minutes }\end{array}$ \\
\hline $10-11-91$ & $\begin{array}{l}962.9-963.8 \mathrm{~m} \\
(3159-3162 \mathrm{ft})\end{array}$ & Drill bit hit void in this interval; lost complete returns \\
\hline $10-14-91$ & $\begin{array}{l}948.5-967.4 \mathrm{~m} \\
(3112-3174 \mathrm{ft})\end{array}$ & Tight spot, hole was reamed within this interval \\
\hline $10-16-91$ & $982.1 \mathrm{~m}(3222 \mathrm{ft})$ & Tight hole at this depth, reamed $18.3 \mathrm{~m}(60 \mathrm{ft})$ \\
\hline $10-17-91$ & $1093.6 \mathrm{~m}(3588 \mathrm{ft})$ & ER-12-1 reaches TD \\
\hline 10-24-91 & $\begin{array}{l}973.8-976.9 \mathrm{~m} \\
(3195-3205 \mathrm{ft})\end{array}$ & Ream tight hole, then trip in to TD \\
\hline $10-28-91$ & $1001.0 \mathrm{~m}(3284 \mathrm{ft})$ & Tagged fill with core barrel \\
\hline $10-29-91$ & $652.3 \mathrm{~m}(2140 \mathrm{ft})$ & Hit bridge with tubing \\
\hline 10-30-91 & $\begin{array}{l}627.6-634.0 \mathrm{~m} \\
(2059-2080 \mathrm{ft})\end{array}$ & Placed cement plug in washout \\
\hline $\mid 1-12-91$ & $549.2 \mathrm{~m}(1802 \mathrm{ft})$ & Caliper tool on run $\# 5$ hits bridge \\
\hline
\end{tabular}


Table 8. Completion and Well Development Fluids and Materials Injected into ER-12-1.

\begin{tabular}{|c|c|c|c|}
\hline Date & Quantity (m ${ }^{3}$ (bbls)) & Fluid/Material & Comments/Activity \\
\hline $10-22-91$ & $76(480)$ & Water & Well Development \\
\hline $10-23-91$ & $478(3000)$ & $\begin{array}{l}\text { Water w/100 lb } \\
\text { Sodium-tetraphosphate }\end{array}$ & Well Development \\
\hline $10-24-91$ & $229(1440)$ & $\begin{array}{l}\text { Water } w / 450 \mathrm{lb} \\
\text { Sodium-tetraphosphate }\end{array}$ & Well Development \\
\hline $10-25-91$ & $1(6)$ & Soap & Well Development \\
\hline $10-25-91$ & $153(960)$ & Water & Well Development \\
\hline $10-30-91$ & $19(120)$ & Water & Well Development \\
\hline $01-13-92$ & $96(600)$ & Mud & Clean hole/remove fill \\
\hline $01-14-92$ & $153(960)$ & Mud & Clean hole/remove fill \\
\hline $01-15-92$ & $114(720)$ & Mud & Clean hole/remove fill \\
\hline $01-16-92$ & $96(600)$ & Mud & Clean hole/remove fill \\
\hline $01-16-92$ & $38(240)$ & Water & Clean hole/remove fill \\
\hline $01-17-92$ & $19(120)$ & Mud & Clean hole/remove fill \\
\hline $01-21-92$ & $134(840)$ & Mud & Clean hole/remove fill \\
\hline $01-22-92$ & $76(480)$ & Water & Condition hole \\
\hline $01-23-92$ & $153(960)$ & Polymer & Condition hole \\
\hline $01-24-92$ & $38(240)$ & Water & Condition hole \\
\hline $01-24-92$ & $19(120)$ & Polymer & Condition hole \\
\hline $01-25-92$ & $133(840)$ & Water & Cement Job/set casing \\
\hline $01-26-92$ & $19(120)$ & Water & Cement Job/set casing \\
\hline $01-27-92$ & $60(380)$ & Water & Cement Job/set casing \\
\hline $01-28-92$ & $19(120)$ & Water & Cement Job/set casing \\
\hline $01-29-92$ & $38(240)$ & Water & Cement Job/set casing \\
\hline $01-29-92$ & $57(360)$ & Polymer & Cement Job/set casing \\
\hline $01-30-92$ & $134(840)$ & Water & Cement Job/set casing \\
\hline $01-31-92$ & $38(240)$ & Water & Cement Job/set casing \\
\hline $02-21-92$ & $153(960)$ & Water & Well Development \\
\hline $02-24-92$ & $38(240)$ & Water & Well Development \\
\hline $02-26-92$ & $19(120)$ & Water & Well Development \\
\hline $08-26-92$ & $57(360)$ & Water & Well Development \\
\hline $08-27-92$ & $32(200)$ & Water & Well Development \\
\hline $08-28-92$ & $96(600)$ & Water & Well Development \\
\hline $08-30-92$ & $19(120)$ & Water & Well Development \\
\hline $08-31-92$ & $166(1040)$ & Water & Well Development \\
\hline $09-01-92$ & $129(810)$ & Water & Well Development \\
\hline $09-03-92$ & $32(200)$ & $\begin{array}{l}\text { Sodium Acid } \\
\text { Pyrophosphate }\end{array}$ & Well Development \\
\hline $09-04-92$ & $32(200)$ & $\begin{array}{l}\text { Sodium Acid } \\
\text { Pyrophosphate }\end{array}$ & Well Development \\
\hline $09-09-92$ & $48(300)$ & $\begin{array}{l}\text { Sodium Acid } \\
\text { Pyrophosphate }\end{array}$ & Well Development \\
\hline $09-10-92$ & $19(120)$ & Water & Well Development \\
\hline
\end{tabular}


Table 9. Rationale for Completion of Intervals in Well ER-12-1.

\begin{tabular}{lll}
\hline Completion Interval & Geologic Formation & Rationale for Completion \\
\hline 516 to $555 \mathrm{~m}(1700$ to $1820 \mathrm{ft})$ & $\begin{array}{l}\text { Upper Simonson or } \\
\text { Lower Guilmette Formation } \\
\text { Upper } 3 \mathrm{~m}(10 \mathrm{ft}) \text { is Eleana Formation }\end{array}$ & $\begin{array}{l}\text { Multiple faults, over } 3000 \text { barrels of mud lost in this interval. } \\
\text { May be highly permeable section }\end{array}$ \\
585 to $598 \mathrm{~m}(1920$ to $1960 \mathrm{ft})$ & Eleana Formation, Unit J & $\begin{array}{l}\text { Representative shaley section of Eleana Formation as } \\
\text { indicated by gamma log and cuttings. Zone should be } \\
\text { representative of a tight shaley section of Eleana Formation }\end{array}$ \\
765 to $790 \mathrm{~m}(2510$ to $2590 \mathrm{ft})$ & Eleana Formation, Unit $\mathrm{J}$ & $\begin{array}{l}\text { Representative silty section of Eleana Formation, purpose } \\
\text { is to evaluate one of the potentially least permeable sections } \\
\text { of the Eleana Formation }\end{array}$ \\
1024 to $1049 \mathrm{~m}(3360$ to $3440 \mathrm{ft})$ & Upper Sevy Dolomite & $\begin{array}{l}\text { Cavernous openings, mud lost, possible permeable section } \\
\text { Representative section of unfaulted tight dolomite, as } \\
\text { determined from dual induction, caliper, epithermal neutron } \\
\text { logs and cuttings }\end{array}$
\end{tabular}


Table 10. Fluid Recovery Record for ER-12-1.

\begin{tabular}{|c|c|c|c|}
\hline Date & Volume $\left(\mathrm{m}^{3}(\mathrm{bbls})\right)$ & Activity & Comments \\
\hline $10-25-91$ & $<16(<100)$ & Air-lifting & $\begin{array}{l}\text { Attempt to develop } \\
\text { well by air-lifting } \\
\text { fluid from hole }\end{array}$ \\
\hline $2-25-92$ & $<16(<100)$ & $\begin{array}{r}\text { Dip-sticking } \\
\text { (dual string) }\end{array}$ & $\begin{array}{l}\text { Attempt to develop } \\
\text { well by air-lifting } \\
\text { fluid from hole }\end{array}$ \\
\hline $2-26$ through $28-92$ & $94(594)$ & Swabbing & $\begin{array}{l}\text { Attempt to develop } \\
\text { well by swabbing } \\
\text { fluid from hole }\end{array}$ \\
\hline $3-5-92$ & $2(15)$ & Pumping & $\begin{array}{l}\text { Attempt to pump well } \\
\text { with } 750 \mathrm{lpm}(200 \\
\text { gpm) pump }\end{array}$ \\
\hline $3-12$ through $15-92$ & $466(2925)$ & $"$ & $\begin{array}{l}\text { Develop well with } \\
56-227 \text { lpm (15-60 } \\
\text { gpm) pump }\end{array}$ \\
\hline $3-16-92$ & $8(50)$ & $"$ & $\begin{array}{l}\text { Test aquifer monitor- } \\
\text { ing equipment }\end{array}$ \\
\hline $3-19-92$ & $16(100)$ & $"$ & $"$ \\
\hline $3-20-92$ & $21(135)$ & $"$ & $"$ \\
\hline $3-24-92$ & $25(156)$ & $"$ & Aquifer testing \\
\hline $4-1$ through 5-92 & $620(3895)$ & $"$ & $"$ \\
\hline $9-14$ through $21-92$ & $438(2755)$ & $\begin{array}{l}\text { Dip-sticking } \\
\text { (dual string) }\end{array}$ & Well development \\
\hline $1-5$ and $6-93$ & $304(1910)$ & Pumping & $\begin{array}{l}\text { Aquifer test } \\
\text { (upper zone only) }\end{array}$ \\
\hline
\end{tabular}


Following initial hydrologic testing and composite geochemical sampling, further well development was conducted by flushing the wellbore with sodium acid pryophosphate to remove bentonite from the borehole then air-lifting approximately $436 \mathrm{~m}^{3}(2738 \mathrm{bbls})$ of fluid from the well (Tables 8). Straddle-packer tests were conducted on the three middle intervals.

To isolate the five slotted intervals, a $139.7 \mathrm{~mm}(51 / 2$ in) diameter casing string was placed within the well. This casing included five sliding sleeves separated by packers (Figure 2), which serve to isolate the five gravel-packed intervals within the well.

After placement of the $139.7 \mathrm{~mm}$ (5 1/2 in) casing with sliding sleeves, the TAM Inc. sliding sleeve shifter tool was used to close all sleeves, and then open the uppermost sleeve. This effectively sealed off the lower four intervals from the wellbore. A submersible pump was then placed within the well and a 24-hour aquifer test was conducted on the isolated upper interval. Prior to the conclusion of the pumping phase of the 24-hour aquifer test, geochemical samples were obtained from the fluids produced from the uppermost zone within the well.

An outline of the sequence of events during drilling, completion, development and testing of ER-12-1 is presented in Appendix D of this report.

According to fluid records supplied by REECo and RSN, a total of $8423 \mathrm{~m}^{3}(52,920)$ barrels of fluids were delivered to the ER-12-1 well site. Approximately $620 \mathrm{~m}^{3}$ (3895 barrels) of fluid were removed from the well and discharged to land surface during aquifer testing. A total of $6270 \mathrm{~m}^{3}$ (39,370 barrels) of used drilling and development fluids were removed from the mudpit at ER-12-1 and hauled by truck to a disposal site. Approximately $318 \mathrm{~m}^{3}$ (2000 barrels) of fluid were left in the sump at ER-12-1. Neglecting losses from the sump by evaporation and dilution of drilling fluids by formation water, $1218 \mathrm{~m}^{3}$ (7655 barrels) of unrecovered fluids remain in ER-12-1.

\section{GEOLOGY OF ER-12-1 BASED ON MACROSCOPIC OBSERVATIONS}

\section{Surface Geology}

The geologic setting for ER-12-1 is different from typical weapons-related sites in that the hole was drilled almost entirely through Paleozoic sedimentary rocks. Figure 3 is a geologic map of the ER-12-1 area, which was extracted from the "Geologic Map of the Rainier Mesa Quadrangle, Nye County, Nevada" (Gibbons et al., 1963). While the geologic interpretations presented in this map are rather old and in some cases changed since publication, the gross geologic characteristics are still viable. Table 11 correlates the old stratigraphic nomenclature used on this map with the more recent nomenclature used elsewhere in this report.

As can be noted from the map, the geology is quite complex due to the extensive faulting (thrust and normal) the area has undergone. Another complication in the geologic interpretation is that compared to the Tertiary volcanic rocks of the NTS, the Paleozoic rocks are less studied, and therefore less familiar to the general NTS geologic community.

Figure 3 illustrates some of the major geologic features of the area. Stockade Wash Road runs through Tongue Wash, which is an erosional feature superimposed upon the Mesozoic Tongue Wash 


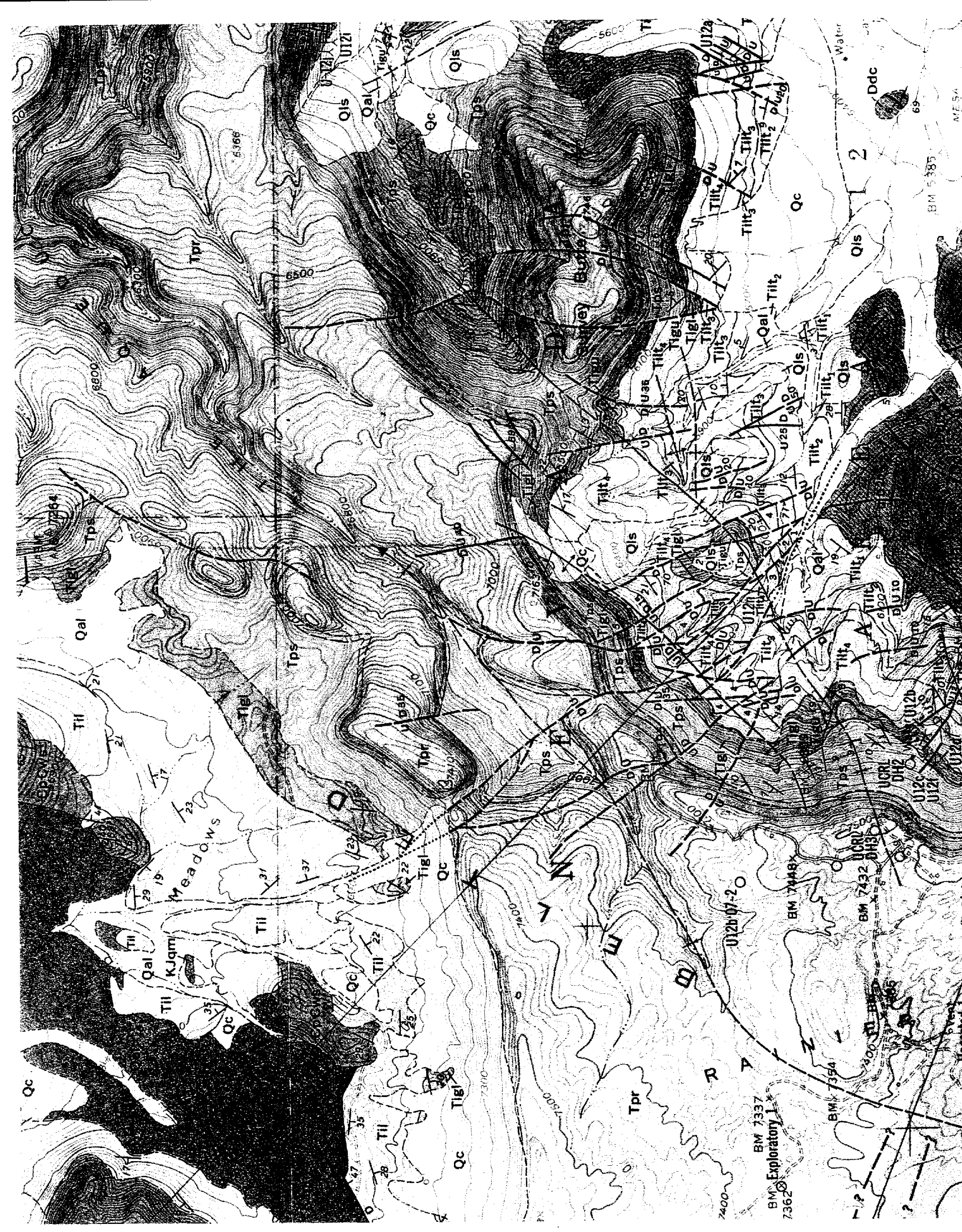

Figure 3. Geologic map of the ER-12-1 vicinity. (Extracted from G 

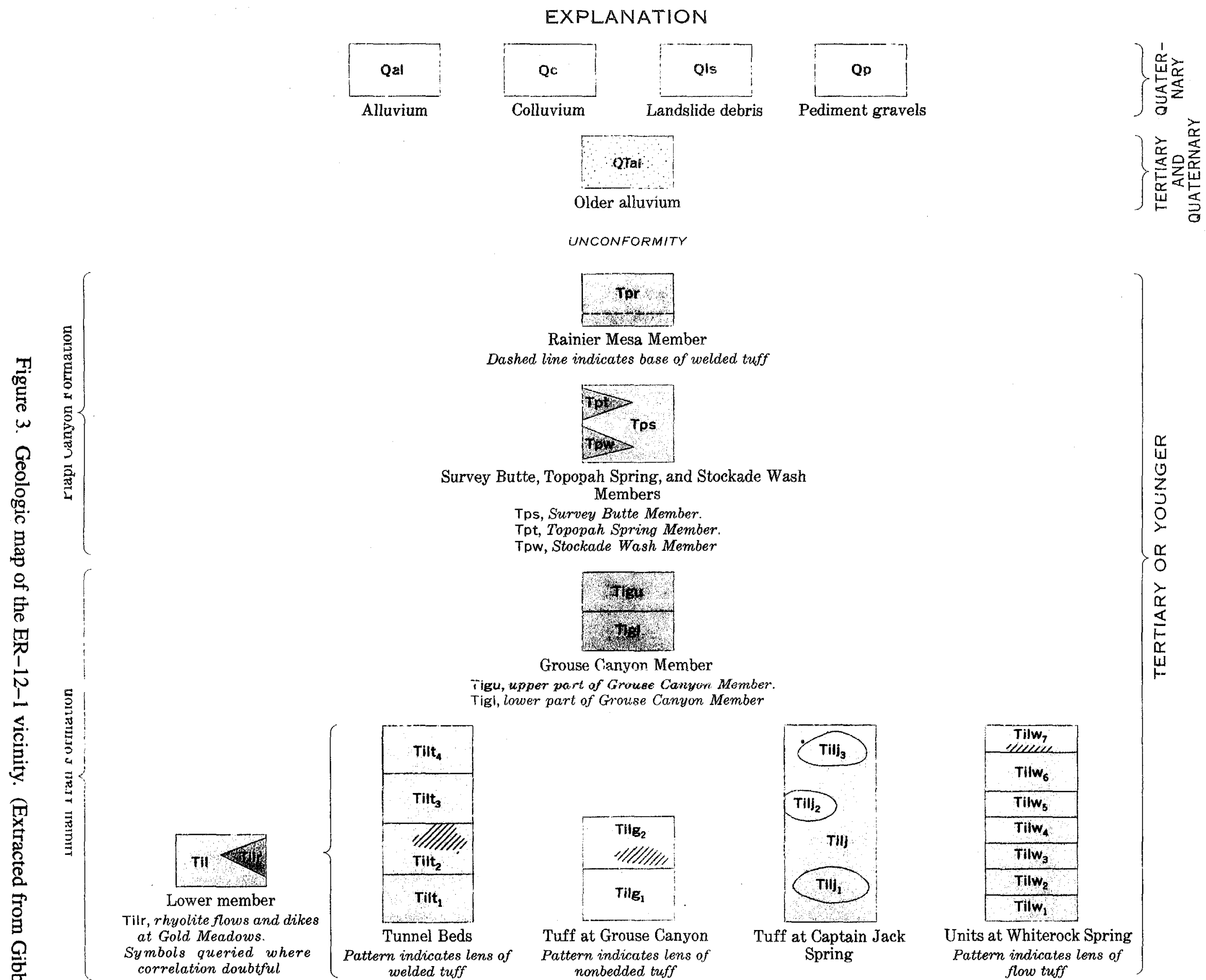

UNCONFORMITY

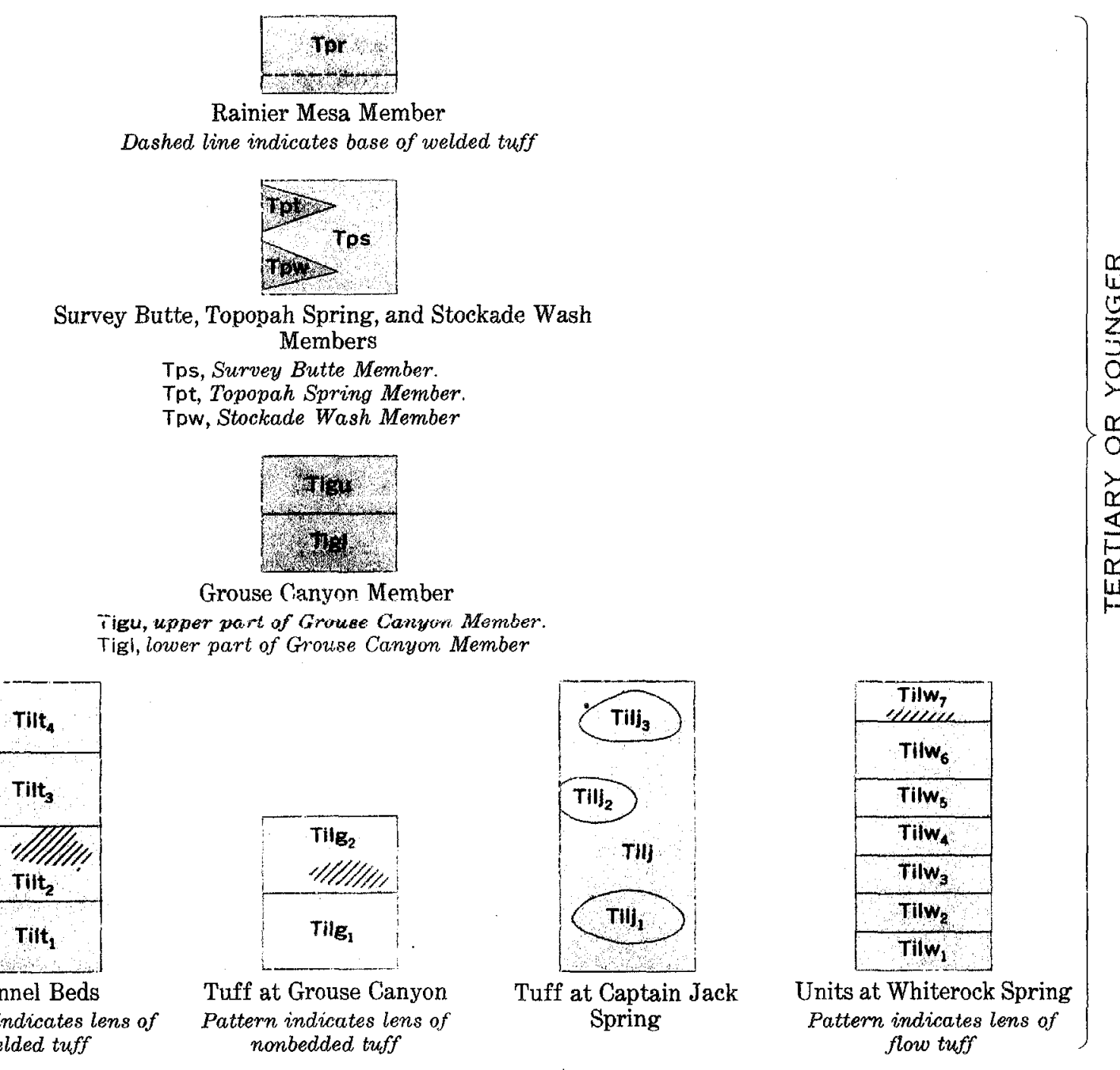
nombedded tuff flow tuff 
Table 12. Sample Disposition Log for ER-12-1.

\begin{tabular}{|c|c|c|c|}
\hline Sample Type & Depth Interval & $\begin{array}{l}\text { Receiving Lab } \\
\text { or Location }\end{array}$ & $\begin{array}{l}\text { Preservation or } \\
\text { Storage Container }\end{array}$ \\
\hline $\begin{array}{l}\text { Washed drill } \\
\text { cuttings }\end{array}$ & $\begin{array}{l}3.05 \text { to } 1093.6 \mathrm{~m} \\
(10 \text { to } 3588 \mathrm{ft}) \\
\text { at } 3.05 \mathrm{~m}(10 \mathrm{ft}) \\
\text { intervals }\end{array}$ & $\begin{array}{l}\text { USGS Core Library } \\
\text { Mercury, NV }\end{array}$ & $\begin{array}{l}\text { Cardboard sample } \\
\text { boxes }\end{array}$ \\
\hline $\begin{array}{l}\text { Unwashed drill } \\
\text { cuttings with } \\
\text { custody seal }\end{array}$ & $\begin{array}{l}3.05 \text { to } 1093.6 \mathrm{~m} \\
(10 \text { to } 3588 \mathrm{ft}) \\
\text { at } 3.05 \mathrm{~m}(10 \mathrm{ft}) \\
\text { intervals }\end{array}$ & $\begin{array}{l}\text { USGS Core Library } \\
\text { Mercury, NV }\end{array}$ & $\begin{array}{l}\text { One-pint ice cream } \\
\text { cartons }\end{array}$ \\
\hline $\begin{array}{l}\text { Washed drill } \\
\text { cuttings "Paleo" } \\
\text { samples }\end{array}$ & $\begin{array}{l}15.2 \text { to } 1082.0 \mathrm{~m} \\
(50 \text { to } 3550 \mathrm{ft}) \\
\text { at } 15.2 \mathrm{~m}(50 \mathrm{ft}) \\
\text { intervals }\end{array}$ & $\begin{array}{l}\text { A. G. Harris } \\
\text { USGS MS } 970 \\
\text { Reston, VA } 22092\end{array}$ & $\begin{array}{l}\text { One-gallon } \\
\text { metal cans }\end{array}$ \\
\hline Core & $\begin{array}{l}543.7 \text { to } 543.8 \mathrm{~m} ? \\
(1783.8 \text { to } 1784 \mathrm{ft} ?)\end{array}$ & $\begin{array}{l}\text { USGS Core Library } \\
\text { Mercury, NV }\end{array}$ & $\begin{array}{l}\text { Cardboard core } \\
\text { boxes }\end{array}$ \\
\hline Core & $\begin{array}{l}751.0-754.1 \mathrm{~m} \\
(2464-2962.5 \mathrm{ft})\end{array}$ & $\begin{array}{l}\text { USGS Core Library } \\
\text { Mercury, NV }\end{array}$ & $\begin{array}{l}\text { Cardboard core } \\
\text { boxes }\end{array}$ \\
\hline Core & $\begin{array}{l}895.2-903.0 \mathrm{~m} \\
(2937-2962.5 \mathrm{ft})\end{array}$ & $\begin{array}{l}\text { USGS Core Library } \\
\text { Mercury, NV }\end{array}$ & $\begin{array}{l}\text { Cardboard core } \\
\text { boxes }\end{array}$ \\
\hline $\begin{array}{l}\text { Polished } \\
\text { thin sections }\end{array}$ & $\begin{array}{l}\text { (See Table } 14 \text { for } \\
\text { list of depths) }\end{array}$ & $\begin{array}{l}\text { RSN Geology } \\
\text { Bldg. } 155 \\
\text { Mercury, NV }\end{array}$ & Boxes \\
\hline $\begin{array}{l}\text { Polished } \\
\text { thin sections }\end{array}$ & $1091.2 \mathrm{~m}(3580 \mathrm{ft})$ & $\begin{array}{l}\text { R. G. Warren } \\
\text { LANL, MS F659 } \\
\text { Los Alamos, NM }\end{array}$ & Box \\
\hline $\begin{array}{l}\text { Standard covered } \\
\text { thin sections }\end{array}$ & $\begin{array}{l}15.2 \text { to } 1082.0 \mathrm{~m} \\
(50 \text { to } 3550 \mathrm{ft})\end{array}$ & $\begin{array}{l}\text { J. C. Cole } \\
\text { USGS, MS } 913 \\
\text { Denver, CO }\end{array}$ & Boxes \\
\hline
\end{tabular}

Drilling operations and subsequent sampling for this drillhole have been extensively documented, including the implementation of a chain-of-custody procedure (with samples sealed on location), and photo-documentation.

\section{Stratigraphy and Lithology of ER-12-1}

ER-12-1 penetrated $1093.6 \mathrm{~m}$ (3588 ft) of sedimentary rocks ranging in age from Ordovician to Mississippian, minor Cretaceous intrusive rock and surficial gravel. Table 19 lists the pre-Tertiary stratigraphic nomenclature used in this report. Table 20 is the stratigraphic $\log$ for ER-12-1. These rocks have been deformed by both Mesozoic compression and Tertiary extension. This combined structural activity has resulted in a complex shuffling of the stratigraphic sequence in the vicinity of ER-12-1. Units encountered at ER-12-1 were initially assigned to formations primarily on the basis of lithologic characteristics (Table 21), but final assignment was made on the basis of paleontologic studies 
Table 11. Correlation of Stratigraphic Nomenclature used in this Report with that used in Figure 3.

\begin{tabular}{ll}
\hline $\begin{array}{c}\text { Figure } 3 \\
\text { (adapted from Gibbons et al., 1963) }\end{array}$ & \multicolumn{1}{c}{$\begin{array}{c}\text { This Report } \\
\text { (adapted from Cole, 1991) }\end{array}$} \\
\hline Qal - Alluvium & QTa - Alluvium \\
PMe - Eleana Formation (Units A-J) & MDe - Eleana Formation (Units A-J) \\
Dd (Units A-C) - Dolomite & DSs - Sevy Dolomite \\
DSO1 - Dolomitic Limestone & \\
\hline
\end{tabular}

fault. Rainier Mesa is evident in Figure 3, and is indicated where the Tertiary Rainier Mesa Tuff caps the less resistant, older underlying units along the western and northern margins of the map. The Eleana Range, a ridge of mostly Paleozoic sediments, is best defined in the southeast corner of Figure 3, where the Devonian to Mississippian rocks of the Eleana Formation predominate.

\section{Sample Inventories and Additional Data}

Cuttings and core samples were collected during the drilling phase of ER-12-1. Drill cuttings samples were collected by the roughneck at $3.04 \mathrm{~m}(10 \mathrm{ft})$ intervals. From the same depth intervals a custody-sealed sample was also collected and placed in $0.47 \mathrm{~L}$ (one-pint) containers. At $15.24 \mathrm{~m}$ (50 ft) intervals, a $3.8 \mathrm{~L}$ (one-gallon) metal container was filled with sample cuttings to be used for micro-paleontologic studies. Table 12 lists the sample types available from ER-12-1 and the archival location or custodian. Cores were attempted at four different depth intervals but only three of the core runs produced core. Table 13 lists the cored intervals, recoveries, and core barrels used.

Thin sections were made by REECo from the drill cuttings at selected depths. Polished thin sections were made for RSN, and a set of standard covered thin sections were made for the USGS at $15.24 \mathrm{~m}(50 \mathrm{ft})$ intervals throughout the hole. Table 14 shows the depth, sample number, lithology, and formation of each of the 15 polished thin sections.

X-ray diffraction analysis was performed on drill cuttings samples from four depths to identify mineral constituents. The percentages of the various minerals in each sample are listed in Table 15.

Physical properties (unconfined compressive strength, bulk density, and porosity) were measured on several globe-basket samples from approximately $754.4 \mathrm{~m}(2475 \mathrm{ft}) \mathrm{depth}$. Table 16 contains these data along with the lithology of each sample.

A downhole camera was run from the surface to $486.3 \mathrm{~m}$ (1596ft) in ER-12-1. Features observed include casing, fractures, washouts, and fluid level. A problem was encountered due to the camera overheating, which resulted in poor resolution over several intervals. A summary of observations from the downhole camera run are presented in Table 17.35-mm slides were taken to document selected equipment, activities, and all the samples. A list of these can be found in Table 18. 
Table 12. Sample Disposition Log for ER-12-1.

\begin{tabular}{|c|c|c|c|}
\hline Sample Type & Depth Interval & $\begin{array}{l}\text { Receiving Lab } \\
\text { or Location }\end{array}$ & $\begin{array}{l}\text { Preservation or } \\
\text { Storage Container }\end{array}$ \\
\hline $\begin{array}{l}\text { Washed drill } \\
\text { cuttings }\end{array}$ & $\begin{array}{l}3.05 \text { to } 1093.6 \mathrm{~m} \\
(10 \text { to } 3588 \mathrm{ft}) \\
\text { at } 3.05 \mathrm{~m}(10 \mathrm{ft}) \\
\text { intervals }\end{array}$ & $\begin{array}{l}\text { USGS Core Library } \\
\text { Mercury, NV }\end{array}$ & $\begin{array}{l}\text { Cardboard sample } \\
\text { boxes }\end{array}$ \\
\hline $\begin{array}{l}\text { Unwashed drill } \\
\text { cuttings with } \\
\text { custody seal }\end{array}$ & $\begin{array}{l}3.05 \text { to } 1093.6 \mathrm{~m} \\
(10 \text { to } 3588 \mathrm{ft}) \\
\text { at } 3.05 \mathrm{~m}(10 \mathrm{ft}) \\
\text { intervals }\end{array}$ & $\begin{array}{l}\text { USGS Core Library } \\
\text { Mercury, NV }\end{array}$ & $\begin{array}{l}\text { One-pint ice cream } \\
\text { cartons }\end{array}$ \\
\hline $\begin{array}{l}\text { Washed drill } \\
\text { cuttings "Paleo" } \\
\text { samples }\end{array}$ & $\begin{array}{l}15.2 \text { to } 1082.0 \mathrm{~m} \\
(50 \text { to } 3550 \mathrm{ft}) \\
\text { at } 15.2 \mathrm{~m}(50 \mathrm{ft}) \\
\text { intervals }\end{array}$ & $\begin{array}{l}\text { A. G. Harris } \\
\text { USGS MS } 970 \\
\text { Reston, VA } 22092\end{array}$ & $\begin{array}{l}\text { One-gallon } \\
\text { metal cans }\end{array}$ \\
\hline Core & $\begin{array}{l}543.7 \text { to } 543.8 \mathrm{~m} ? \\
\text { (1783.8 to } 1784 \mathrm{ft} ?)\end{array}$ & $\begin{array}{l}\text { USGS Core Library } \\
\text { Mercury, NV }\end{array}$ & $\begin{array}{l}\text { Cardboard core } \\
\text { boxes }\end{array}$ \\
\hline Core & $\begin{array}{l}751.0-754.1 \mathrm{~m} \\
(2464-2962.5 \mathrm{ft})\end{array}$ & $\begin{array}{l}\text { USGS Core Library } \\
\text { Mercury, NV }\end{array}$ & $\begin{array}{l}\text { Cardboard core } \\
\text { boxes }\end{array}$ \\
\hline Core & $\begin{array}{l}895.2-903.0 \mathrm{~m} \\
(2937-2962.5 \mathrm{ft})\end{array}$ & $\begin{array}{l}\text { USGS Core Library } \\
\text { Mercury, NV }\end{array}$ & $\begin{array}{l}\text { Cardboard core } \\
\text { boxes }\end{array}$ \\
\hline $\begin{array}{l}\text { Polished } \\
\text { thin sections }\end{array}$ & $\begin{array}{l}\text { (See Table } 14 \text { for } \\
\text { list of depths) }\end{array}$ & $\begin{array}{l}\text { RSN Geology } \\
\text { Bldg. 155 } \\
\text { Mercury, NV }\end{array}$ & Boxes \\
\hline $\begin{array}{l}\text { Polished } \\
\text { thin sections }\end{array}$ & $1091.2 \mathrm{~m}(3580 \mathrm{ft})$ & $\begin{array}{l}\text { R. G. Warren } \\
\text { LANL, MS F659 } \\
\text { Los Alamos, NM }\end{array}$ & Box \\
\hline $\begin{array}{l}\text { Standard covered } \\
\text { thin sections }\end{array}$ & $\begin{array}{l}15.2 \text { to } 1082.0 \mathrm{~m} \\
(50 \text { to } 3550 \mathrm{ft})\end{array}$ & $\begin{array}{l}\text { J. C. Cole } \\
\text { USGS, MS } 913 \\
\text { Denver, CO }\end{array}$ & Boxes \\
\hline
\end{tabular}

Drilling operations and subsequent sampling for this drillhole have been extensively documented, including the implementation of a chain-of-custody procedure (with samples sealed on location), and photo-documentation.

\section{Stratigraphy and Lithology of ER-12-1}

ER-12-1 penetrated $1093.6 \mathrm{~m}$ ( $3588 \mathrm{ft}$ ) of sedimentary rocks ranging in age from Ordovician to Mississippian, minor Cretaceous intrusive rock and surficial gravel. Table 19 lists the pre-Tertiary stratigraphic nomenclature used in this report. Table 20 is the stratigraphic log for ER-12-1. These rocks have been deformed by both Mesozoic compression and Tertiary extension. This combined structural activity has resulted in a complex shuffling of the stratigraphic sequence in the vicinity of ER-12-1. Units encountered at ER-12-1 were initially assigned to formations primarily on the basis of lithologic characteristics (Table 21), but final assignment was made on the basis of paleontologic studies completed after drilling ceased. Detailed descriptions of cored intervals are presented in Tables 22, 23, and 24 . Table 25 lists other nearby holes that penetrated pre-Tertiary rocks. 
Table 13. ER-12-1 Core Inventory Record.

\begin{tabular}{|c|c|c|c|c|c|c|c|c|}
\hline $\begin{array}{l}\text { Core } \\
\text { No. }\end{array}$ & Date & $\begin{array}{l}\text { Archive } \\
\text { Box No. }\end{array}$ & Cored Interval & Cut & Recovered & Percent & $\begin{array}{l}\text { Core Barrel } \\
\text { Recovery }\end{array}$ & Remarks \\
\hline 1 & $08-03-91$ & 1 & $\begin{array}{l}539.2 \text { to } 543.8 \mathrm{~m} \\
(1769 \text { to } 1784 \mathrm{ft})\end{array}$ & $\begin{array}{r}4.6 \mathrm{~m} \\
(15 \mathrm{ft})\end{array}$ & $\begin{array}{l}0.06 \mathrm{~m} \\
(0.2 \mathrm{ft})\end{array}$ & 1.3 & $\begin{array}{l}\text { Conventional } \\
\text { core barrel }\end{array}$ & Core barrel jammed \\
\hline 2 & $09-03-91$ & N/A & $\begin{array}{l}548.0 \text { to } 556.3 \mathrm{~m} \\
(1798 \text { to } 1825 \mathrm{ft})\end{array}$ & $\begin{array}{r}8.2 \mathrm{~m} \\
(27 \mathrm{ft})\end{array}$ & $\begin{array}{l}0 \mathrm{~m} \\
(0)\end{array}$ & 0 & $\begin{array}{l}\text { Marine } \\
\text { core barrel }\end{array}$ & \\
\hline 3 & $09-23-91$ & $1 \& 2$ & $\begin{array}{l}751.0 \text { to } 757.7 \mathrm{~m} \\
(2464 \text { to } 2486 \mathrm{ft})\end{array}$ & $\begin{array}{r}6.7 \mathrm{~m} \\
(22 \mathrm{ft})\end{array}$ & $\begin{array}{r}3.0 \mathrm{~m} \\
(10 \mathrm{ft})\end{array}$ & 45.5 & $\begin{array}{l}\text { Marine } \\
\text { core barrel }\end{array}$ & $\begin{array}{l}\text { Left bit cones in } \\
\text { hole }\end{array}$ \\
\hline 4 & $10-08-91$ & $\begin{array}{l}3,4,5 \\
6, \& 7\end{array}$ & $\begin{array}{l}895.2 \text { to } 903.1 \mathrm{~m} \\
(2937 \text { to } 2963 \mathrm{ft})\end{array}$ & $\begin{array}{r}7.9 \mathrm{~m} \\
(26 \mathrm{ft})\end{array}$ & $\begin{array}{l}7.8 \mathrm{~m} \\
(25.5 \mathrm{ft})\end{array}$ & 98 & $\begin{array}{l}\text { Conventional } \\
\text { core barrel }\end{array}$ & Core barrel jammed \\
\hline
\end{tabular}


Table 14. List of Polished Thin Sections Available from Selected ER-12-1 Cuttings Samples. ${ }^{1}$

\begin{tabular}{|c|c|c|c|}
\hline Sample ID No. & Depth & Lithology & Formation \\
\hline $\begin{array}{l}\text { ER-12-1 } \\
730\end{array}$ & $\begin{array}{l}222.5 \mathrm{~m} \\
(730 \mathrm{ft})\end{array}$ & Dolomite & Sevy Dolomite 3 \\
\hline $\begin{array}{l}\text { ER-12-1 } \\
800\end{array}$ & $\begin{array}{l}243.8 \mathrm{~m} \\
(800 \mathrm{ft})\end{array}$ & Dolomite & Antelope Valley Limestone \\
\hline $\begin{array}{l}\text { ER-12-1 } \\
1000\end{array}$ & $\begin{array}{l}304.8 \mathrm{~m} \\
(1000 \mathrm{ft})\end{array}$ & Dolomite & Sevy Dolomite \\
\hline $\begin{array}{l}\text { ER-12-1 } \\
1050\end{array}$ & $\begin{array}{l}320.0 \mathrm{~m} \\
(1050 \mathrm{ft})\end{array}$ & Calcareous Siltstone & $\begin{array}{l}\text { Eleana Formation, } \\
\text { Unit } \mathrm{J}(?)\end{array}$ \\
\hline $\begin{array}{l}\text { ER-12-1 } \\
1080\end{array}$ & $\begin{array}{l}329.2 \mathrm{~m} \\
(1080 \mathrm{ft})\end{array}$ & Siltstone & $\begin{array}{l}\text { Eleana Formation, } \\
\text { Unit J (?) }\end{array}$ \\
\hline $\begin{array}{l}\text { ER-12-1 } \\
1140\end{array}$ & $\begin{array}{l}347.5 \mathrm{~m} \\
(1140 \mathrm{ft})\end{array}$ & Siltstone & $\begin{array}{l}\text { Eleana Formation, } \\
\text { Unit J (?) }\end{array}$ \\
\hline $\begin{array}{l}\text { ER-12-1 } \\
1650\end{array}$ & $\begin{array}{l}502.9 \mathrm{~m} \\
(1650 \mathrm{ft})\end{array}$ & Siltstone & $\begin{array}{l}\text { Eleana Formation, } \\
\text { Unit } J(?)\end{array}$ \\
\hline $\begin{array}{l}\text { ER-12-1 } \\
1800\end{array}$ & $\begin{array}{l}548.6 \mathrm{~m} \\
(1800 \mathrm{ft})\end{array}$ & Dolomite & $\begin{array}{l}\text { Upper Simonson or } \\
\text { Lower Guilmette Formation }\end{array}$ \\
\hline $\begin{array}{l}\text { ER-12-1 } \\
1960\end{array}$ & $\begin{array}{l}597.4 \mathrm{~m} \\
(1960 \mathrm{ft})\end{array}$ & Quartzite & $\begin{array}{l}\text { Eleana Formation, } \\
\text { Unit } \mathrm{J}(?)\end{array}$ \\
\hline $\begin{array}{l}\text { ER-12-1 } \\
2240\end{array}$ & $\begin{array}{l}682.8 \mathrm{~m} \\
(2240 \mathrm{ft})\end{array}$ & Shale & $\begin{array}{l}\text { Eleana Formation, } \\
\text { Unit J (?) }\end{array}$ \\
\hline $\begin{array}{l}\text { ER-12-1 } \\
2370\end{array}$ & $\begin{array}{l}722.4 \mathrm{~m} \\
(2370 \mathrm{ft})\end{array}$ & Quartzite & $\begin{array}{l}\text { Eleana Formation, } \\
\text { Unit J }\end{array}$ \\
\hline $\begin{array}{l}\text { ER-12-1 } \\
2850\end{array}$ & $\begin{array}{l}868.7 \mathrm{~m} \\
(2850 \mathrm{ft})\end{array}$ & Calcareous Siltstone & $\begin{array}{l}\text { Eleana Formation, } \\
\text { Unit } \mathrm{J}(?)\end{array}$ \\
\hline $\begin{array}{l}\text { ER-12-1 } \\
2930\end{array}$ & $\begin{array}{l}893.1 \mathrm{~m} \\
(2930 \mathrm{ft})\end{array}$ & Dolomite & $\begin{array}{l}\text { Upper Sevy or Lower } \\
\text { Simonson Dolomite }\end{array}$ \\
\hline $\begin{array}{l}\text { ER-12-1 } \\
3570\end{array}$ & $\begin{array}{l}1088.1 \mathrm{~m} \\
(3570 \mathrm{ft})\end{array}$ & Dolomite & $\begin{array}{l}\text { Upper Sevy or Lower } \\
\text { Simonson Dolomite }\end{array}$ \\
\hline $\begin{array}{l}\text { ER- } 12-1^{2} \\
3580\end{array}$ & $\begin{array}{l}1091.2 \mathrm{~m} \\
(3580 \mathrm{ft})\end{array}$ & $\begin{array}{l}\text { Biotite } \\
\text { Lamprophyre }\end{array}$ & $\begin{array}{l}\text { Lamprophyre Dike, (Middle } \\
\text { Cretaceous) intrudes Sevy } \\
\text { Dolomite }\end{array}$ \\
\hline
\end{tabular}

\footnotetext{
${ }^{1}$ An independent set of thin sections has been established by Jim Cole. USGS-Denver, for the entire borehole at 15.2 $\mathrm{m}(50 \mathrm{ft})$ depth intervals.

${ }^{2}$ A polished thin section was made for the purpose of microprobe work by R. G. Warren, LANL. of the $1091.2 \mathrm{~m}$ (3580 ft) cuttings sample.

${ }^{3}$ Stratigraphic assignment based on paleontological investigations by A.G. Harris. USGS - Reston, VA.
} 


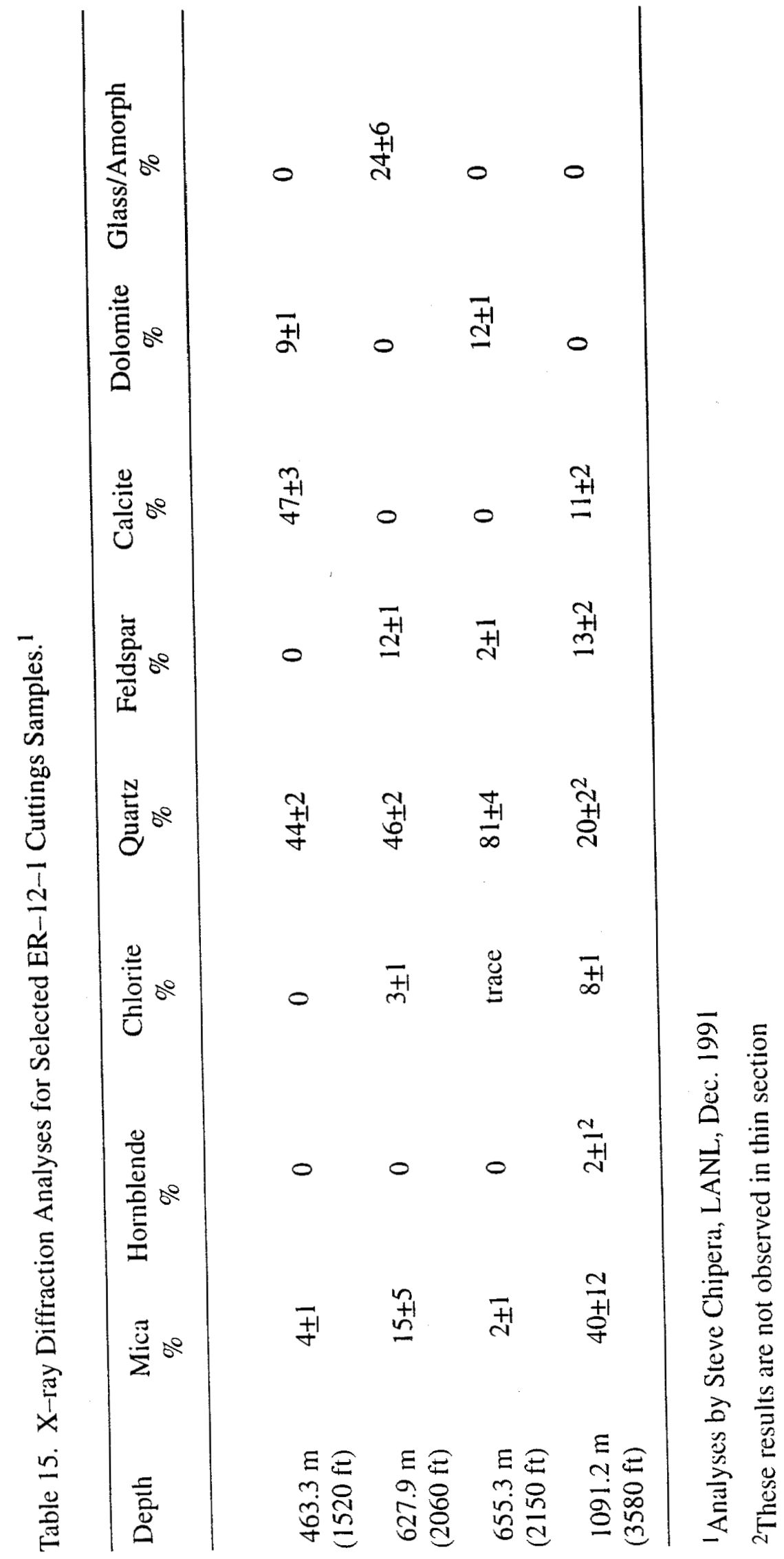


Table 16. Physical Properties Measurements on Selected ER-12-1 Samples.

\begin{tabular}{|c|c|c|c|c|}
\hline $\begin{array}{l}\text { Sample }{ }^{1} \\
\text { Depth }\end{array}$ & $\begin{array}{l}\text { Unconfined } \\
\text { Compressive } \\
\text { Strength (psi) }\end{array}$ & $\begin{array}{l}\text { Bulk } \\
\text { Density } \\
(\mathrm{g} / \mathrm{cc})\end{array}$ & $\begin{array}{c}\text { Apparent } \\
\text { Porosity } \\
\text { to Water }(\%)\end{array}$ & Lithology \\
\hline $\begin{array}{r}754.4 \mathrm{~m} \\
(2475 \mathrm{ft})\end{array}$ & 8220 & NA & NA & Argillaceous quartzite \\
\hline $\begin{array}{r}754.4 \mathrm{~m} \\
(2475 \mathrm{ft})\end{array}$ & $3720^{2}$ & NA & NA & Argillaceous quartzite \\
\hline $\begin{array}{r}754.4 \mathrm{~m} \\
(2475 \mathrm{ft})\end{array}$ & 4160 & NA & NA & Argillaceous quartzite \\
\hline $\begin{array}{r}754.4 \mathrm{~m} \\
(2475 \mathrm{ft})\end{array}$ & 11820 & NA & NA & Argillaceous quartzite \\
\hline $\begin{array}{r}754.4 \mathrm{~m} \\
(2475 \mathrm{ft})\end{array}$ & 7300 & NA & NA & Argillaceous quartzite \\
\hline $\begin{array}{r}754.4 \mathrm{~m} \\
(2475 \mathrm{ft})\end{array}$ & $\mathrm{NA}$ & $2.68^{3}$ & 0.4 & Argillaceous quartzite \\
\hline $\begin{array}{r}754.4 \mathrm{~m} \\
(2475 \mathrm{ft})\end{array}$ & $\mathrm{NA}$ & $2.67^{3}$ & 0.5 & Argillaceous quartzite \\
\hline
\end{tabular}

Analyses by E. Aamodt, RSN Materials Test Lab, Sept. and Oct., 1991

'Globe-basket samples after Core \#3 (751.0 - $757.7 \mathrm{~m} \mathrm{(2464} \mathrm{-} 2486 \mathrm{ft})$ )

2Sample failed at a vein or healed fracture (?)

${ }^{3}$ Bulk density testing yielded identical results (saturated and unsaturated) indicative of a very low porosity rock

ER-12-1 was spudded in Quaternary/Tertiary alluvium. This alluvium unconformably overlies

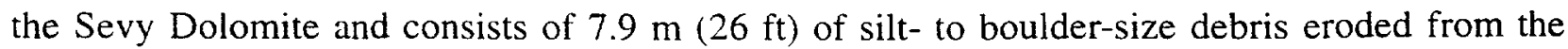
surrounding hills.

The Sevy Dolomite (late Silurian to early Devonian) was encountered in three separate intervals in the ER-12-1 borehole: $7.9-237.8 \mathrm{~m}(26-780 \mathrm{ft}), 274.3-312.4 \mathrm{~m}(900-1025 \mathrm{ft})$, and $975.4-1086.3 \mathrm{~m}(3200-3564 \mathrm{ft})$.

Dolomite assigned to mid to late Devonian upper Simonson Dolomite or lower Guilmette formation were encountered twice during the drilling of ER-12-1. A $29.6 \mathrm{~m}(97 \mathrm{ft})$ thick section of intensely faulted and fractured, light-gray to medium-light-gray dolomite was penetrated from $521.2-550.8 \mathrm{~m}(1710-1807 \mathrm{ft})$. This section lies between two relatively thick intervals of the 
Table 17. Downhole Camera Video Log for ER-12-1 (Surface to $486.3 \mathrm{~m}$ (1596 ft) Interval, (08-21-91).

\begin{tabular}{|c|c|c|}
\hline Tape Time (Hr Min Sec) & Depth & Comments \\
\hline \multicolumn{3}{|l|}{ Tape \#1: Run \#1 } \\
\hline $0: 0: 00$ & N/A & $\begin{array}{l}\text { Start of Tape \#1 and Run \#1;0- } \\
365.8 \mathrm{~m}(0-1200 \mathrm{ft})\end{array}$ \\
\hline $0: 1: 00$ & N/A & Camera at rig floor \\
\hline $0: 2: 00-0: 5: 00$ & N/A & Calibration \\
\hline $0: 6: 00$ & N/A & Going into surface casing \\
\hline $0: 6: 25$ & 0 & Ground level \\
\hline $0: 8: 00$ & 0 & Ground level \\
\hline $0: 13: 22$ & $\begin{array}{l}15.3 \mathrm{~m} \\
(50.3 \mathrm{ft})\end{array}$ & Base of surface casing \\
\hline $0: 14: 15$ & $\begin{array}{l}16.7 \mathrm{~m} \\
(54.8 \mathrm{ft})\end{array}$ & $\begin{array}{l}\text { Change from cement to formation } \\
\text { (Simonson Dolomite) }\end{array}$ \\
\hline $0: 22: 25$ & $\begin{array}{l}36.0 \mathrm{~m} \\
(118 \mathrm{ft})\end{array}$ & Minor hole enlargement \\
\hline $0: 22: 53$ & $\begin{array}{l}37.5 \mathrm{~m} \\
(123 \mathrm{ft})\end{array}$ & Fracture trending N-S (NE-SW fracture also) \\
\hline $0: 24: 19$ & $\begin{array}{l}38.6 \mathrm{~m} \\
(126.5 \mathrm{ft})\end{array}$ & $\begin{array}{l}\text { Intersecting fractures trend SE-NW } \\
\text { and NE-SW }\end{array}$ \\
\hline $0: 25: 20$ & $\begin{array}{l}42.2 \mathrm{~m} \\
(138.5 \mathrm{ft})\end{array}$ & $\begin{array}{l}\text { White reflective calcite(?)-filled fracture on } \\
\text { left side of screen (north side of borehole) }\end{array}$ \\
\hline $0: 26: 23$ & $\begin{array}{l}43.6 \mathrm{~m} \\
(143 \mathrm{ft})\end{array}$ & $\mathrm{N}-\mathrm{S}-$ trending fracture \\
\hline $0: 26: 50$ & $\begin{array}{l}45.1 \mathrm{~m} \\
(148 \mathrm{ft})\end{array}$ & $\mathrm{N}$-S-trending fracture \\
\hline $0: 27: 15$ & $\begin{array}{l}45.7 \mathrm{~m} \\
(150 \mathrm{ft})\end{array}$ & Gyro reading (readings every $15.2 \mathrm{~m}(50 \mathrm{ft})$ ) \\
\hline $0: 31: 12-0: 34: 40$ & $\begin{array}{l}58.5-60.0 \mathrm{~m} \\
(192-197 \mathrm{ft})\end{array}$ & $\begin{array}{l}\text { Adjusting sheave on derrick floor resulting in } \\
\text { negative } 1.2 \mathrm{~m}(4 \mathrm{ft}) \text { depth correction for } 61.0 \\
-221.0 \mathrm{~m}(200-725 \mathrm{ft}) \text { interval }\end{array}$ \\
\hline $0: 36: 20$ & $\begin{array}{l}64.2 \mathrm{~m} \\
(210.5 \mathrm{ft})\end{array}$ & NE-SW-trending fracture \\
\hline $0: 39: 20$ & $\begin{array}{l}70.7 \mathrm{~m} \\
(232 \mathrm{ft})\end{array}$ & NW-SE-trending fracture \\
\hline $0: 40: 13$ & $73.6 \mathrm{~m}$ & $\begin{array}{l}\text { Rough borehole due to breakout in } \\
(241.4 \mathrm{ft}) \text { fractured interval }\end{array}$ \\
\hline $0: 42: 43$ & $\begin{array}{l}77.1 \mathrm{~m} \\
(253 \mathrm{ft})\end{array}$ & Fracture trending N-S \\
\hline $0: 43: 30$ & $\begin{array}{l}78.9 \mathrm{~m} \\
(259 \mathrm{ft})\end{array}$ & Fracture \\
\hline 0:45:00 & $\begin{array}{l}82.6 \mathrm{~m} \\
(271 \mathrm{ft})\end{array}$ & $\begin{array}{l}\text { Borehole enlargement due to N-S and } \\
\text { NE-SW-trending fractures }\end{array}$ \\
\hline
\end{tabular}


Table 17. Downhole Camera Video Log for ER-12-1 (Surface to $486.3 \mathrm{~m}$ (1596 ft) Interval, 08-21-91) (continued).

\begin{tabular}{|c|c|c|}
\hline Tape Time (Hr Min Sec) & Depth & Comments \\
\hline $0: 51: 30$ & $\begin{array}{l}104.2 \mathrm{~m} \\
(342 \mathrm{ft})\end{array}$ & $\begin{array}{l}\text { Fractures with NE-SW hole } \\
\text { enlargement }\end{array}$ \\
\hline 1:00:00 & $\begin{array}{l}121.9 \mathrm{~m} \\
(400 \mathrm{ft})\end{array}$ & $\begin{array}{l}\text { Resolution becoming progressively } \\
\text { worse (camera system overheating?) }\end{array}$ \\
\hline 1:07:07 & $\begin{array}{l}152.4 \\
(500 \mathrm{ft})\end{array}$ & $\begin{array}{l}\text { Gyro reading; video quality is only } \\
\text { marginally useful }\end{array}$ \\
\hline $1: 18: 10$ & $\begin{array}{l}213.4 \mathrm{~m} \\
(700 \mathrm{ft})\end{array}$ & Video is useless \\
\hline 1:19:53 & $\begin{array}{l}221.1 \mathrm{~m} \\
(725.5 \mathrm{ft})\end{array}$ & Max depth for Run \#1 \\
\hline \multicolumn{3}{|l|}{ Tape \#1: Run \#2 } \\
\hline $1: 20: 00$ & $\begin{array}{l}213.4 \mathrm{~m} \\
(700 \mathrm{ft})\end{array}$ & Pulled out of hole to cool camera \\
\hline $1: 20: 30$ & $\begin{array}{l}214.3 \mathrm{~m} \\
(703 \mathrm{ft})\end{array}$ & $\begin{array}{l}\text { Highly reflective fracture fill in } \\
\text { upper left corner }\end{array}$ \\
\hline $1: 22: 20$ & $\begin{array}{l}223.1 \mathrm{~m} \\
(732 \mathrm{ft})\end{array}$ & $\begin{array}{l}\mathrm{N}-\mathrm{S} \text {-trending fracture-controlled } \\
\text { breakout }\end{array}$ \\
\hline 1:22:59 & $\begin{array}{l}227.1 \mathrm{~m} \\
(745 \mathrm{ft})\end{array}$ & $\begin{array}{l}\text { Camera rate has increased to } 6.1 \mathrm{~m} \\
(20 \mathrm{ft}) / \text { minute }\end{array}$ \\
\hline $1: 23: 53$ & $\begin{array}{l}233.6 \mathrm{~m} \\
(766.5 \mathrm{ft})\end{array}$ & Fracture in lower left corner of screen \\
\hline $1: 24: 30$ & $\begin{array}{l}237.7 \mathrm{~m} \\
(780 \mathrm{ft})\end{array}$ & Possible contact, moderate dip \\
\hline $1: 24: 39$ & $\begin{array}{l}239.0 \mathrm{~m} \\
(784 \mathrm{ft})\end{array}$ & $\begin{array}{l}\text { Patches of orange/red staining } \\
\text { exposed behind wall cake }\end{array}$ \\
\hline $1: 25: 00$ & $\begin{array}{l}241.4 \mathrm{~m} \\
(792 \mathrm{ft})\end{array}$ & Base of red stain \\
\hline 1:25:07 & $\begin{array}{l}242.2 \mathrm{~m} \\
(794.5 \mathrm{ft})\end{array}$ & Parabolic fracture trace? \\
\hline $1: 25: 48$ & $\begin{array}{l}245.1 \mathrm{~m} \\
(804 \mathrm{ft})\end{array}$ & $\begin{array}{l}\text { Fracture on lower left corner of } \\
\text { screen }\end{array}$ \\
\hline $1: 26: 00$ & $\begin{array}{l}246.0 \mathrm{~m} \\
(807 \mathrm{ft})\end{array}$ & Reddish stain still seen \\
\hline $1: 26: 57$ & $\begin{array}{l}251.0 \mathrm{~m} \\
(823.5 \mathrm{ft})\end{array}$ & Fracture \\
\hline $1: 27: 20$ & $\begin{array}{l}253.0 \mathrm{~m} \\
(830 \mathrm{ft})\end{array}$ & $\begin{array}{l}\text { Hole enlargement possibly fracture } \\
\text { controlled; one set is N-S, the other E-W }\end{array}$ \\
\hline $1: 28: 25$ & $\begin{array}{l}256.3 \mathrm{~m} \\
(841 \mathrm{ft})\end{array}$ & NW-SE fracture-controlled breakout \\
\hline $1: 29: 23$ & $\frac{259.1 \mathrm{~m}}{(850 \mathrm{ft})}$ & Hole back in gauge \\
\hline
\end{tabular}


Table 17. Downhole Camera Video Log for ER-12-1 (Surface to $486.3 \mathrm{~m}$ (1596 ft) Interval, 08-21-91) (continued).

\begin{tabular}{|c|c|c|}
\hline Tape Time (Hr Min Sec) & Depth & Comments \\
\hline $1: 30: 52$ & $\begin{array}{l}265.8 \mathrm{~m} \\
(872 \mathrm{ft})\end{array}$ & $\begin{array}{l}\text { Orange-red stain behind wall cake } \\
\text { still persists }\end{array}$ \\
\hline $1: 31: 40$ & $\begin{array}{l}266.6 \mathrm{~m} \\
(874.8 \mathrm{ft})\end{array}$ & Fracture-controlled breakout \\
\hline $1: 32: 33$ & $\begin{array}{l}272.2 \mathrm{~m} \\
(893 \mathrm{ft})\end{array}$ & Fractures on right side of screen \\
\hline $1: 33: 00$ & $\begin{array}{l}278.9 \mathrm{~m} \\
(915 \mathrm{ft})\end{array}$ & $\begin{array}{l}\text { Darker-brown-colored planar facets, } \\
\text { possible fractures; camera overheating }\end{array}$ \\
\hline $1: 34: 00$ & $\begin{array}{l}281.9 \mathrm{~m} \\
(925 \mathrm{ft})\end{array}$ & Fractures \\
\hline $1: 34: 14$ & $\begin{array}{l}282.9 \mathrm{~m} \\
(928 \mathrm{ft})\end{array}$ & $\begin{array}{l}\text { Fracture-controlled breakout on upper right } \\
\text { corner of screen; two sets within } 10^{\circ} \text { of N-S }\end{array}$ \\
\hline $1: 34: 50$ & $\begin{array}{l}285.0 \mathrm{~m} \\
(935 \mathrm{ft})\end{array}$ & Possible parabolic fracture traces \\
\hline $1: 36: 35$ & $\begin{array}{l}290.8 \mathrm{~m} \\
(954 \mathrm{ft})\end{array}$ & N-S hairline fracture trace (?) \\
\hline $1: 37: 20$ & $\begin{array}{l}290.5 \mathrm{~m} \\
(953 \mathrm{ft})\end{array}$ & $\begin{array}{l}\text { Possible indistinct bedding on upper } \\
\text { left side of screen }\end{array}$ \\
\hline 1:39:05 & $\begin{array}{l}304.8 \mathrm{~m} \\
(1000 \mathrm{ft})\end{array}$ & Gyro check \\
\hline $1: 40: 48$ & $\begin{array}{l}311.8 \mathrm{~m} \\
(1023 \mathrm{ft})\end{array}$ & Parabolic trace through borehole \\
\hline 1:43:03 & $\begin{array}{l}326.0 \mathrm{~m} \\
(1069.5 \mathrm{ft})\end{array}$ & $\begin{array}{l}\text { Hint of intersecting fracture on left } \\
\text { side of screen }\end{array}$ \\
\hline $1: 43: 25$ & $\begin{array}{l}326.1 \mathrm{~m} \\
(1070 \mathrm{ft})\end{array}$ & Several fracture sets \\
\hline $1: 45: 36$ & $\begin{array}{l}335.0 \mathrm{~m} \\
(1099 \mathrm{ft})\end{array}$ & $\begin{array}{l}\text { Several fracture sets at high angle; } \\
\text { video quality is deteriorating }\end{array}$ \\
\hline $1: 46: 54$ & $\begin{array}{l}339.1 \mathrm{~m} \\
(1112.6 \mathrm{ft})\end{array}$ & $\begin{array}{l}\text { High-angle fracture plane trending } \\
\mathrm{N}-\mathrm{S}\end{array}$ \\
\hline $1: 47: 28$ & $\begin{array}{l}341.7 \mathrm{~m} \\
(1121 \mathrm{ft})\end{array}$ & Spiral traces along borehole \\
\hline $1: 47: 37$ & $\begin{array}{l}342.3 \mathrm{~m} \\
(1123 \mathrm{ft})\end{array}$ & Dipping parabolic trace \\
\hline $1: 47: 58$ & $\begin{array}{l}344.4 \mathrm{~m} \\
(1130 \mathrm{ft})\end{array}$ & High-angle linear fracture (?) trace \\
\hline $1: 48: 10$ & $\begin{array}{l}345.8 \mathrm{~m} \\
(1134.5 \mathrm{ft})\end{array}$ & High-angle linear fracture (?) trace \\
\hline $1: 48: 22$ & $\begin{array}{l}346.9 \mathrm{~m} \\
(1138 \mathrm{ft})\end{array}$ & Parabolic trace dipping SW \\
\hline $1: 49: 19$ & $\begin{array}{l}349.3 \mathrm{~m} \\
(1146 \mathrm{ft})\end{array}$ & Fracture on lower left screen \\
\hline
\end{tabular}


Table 17. Downhole Camera Video Log for ER-12-1 (Surface to $486.3 \mathrm{~m}$ (1596 ft) Interval, 08-21-91) (continued).

\begin{tabular}{|c|c|c|}
\hline Tape Time (Hr Min Sec) & Depth & Comments \\
\hline $1: 49: 30$ & $\begin{array}{l}350.5 \mathrm{~m} \\
(1150 \mathrm{ft})\end{array}$ & Fracture \\
\hline $1: 50: 40$ & $\begin{array}{l}356.3 \mathrm{~m} \\
(1169 \mathrm{ft})\end{array}$ & Fractures on right side of screen \\
\hline 1:51:06 & $\begin{array}{l}359.1 \mathrm{~m} \\
(1178 \mathrm{ft})\end{array}$ & $\begin{array}{l}\text { Partial parabolic ring on right side } \\
\text { of screen }\end{array}$ \\
\hline $1: 52: 26$ & $\begin{array}{l}365.8 \mathrm{~m} \\
(1200 \mathrm{ft})\end{array}$ & $\begin{array}{l}\text { Stop to cool camera for better resolution, } \\
\text { unsuccessful; pull out of hole; end of Tape \#1 }\end{array}$ \\
\hline \multicolumn{3}{|l|}{ Tape \#2, Run \#3 } \\
\hline $0: 0: 00$ & $\begin{array}{l}357.8 \mathrm{~m} \\
(1174 \mathrm{ft})\end{array}$ & Start of tape \\
\hline $0: 0: 36$ & $\begin{array}{l}365.8 \mathrm{~m} \\
(1200 \mathrm{ft})\end{array}$ & Run \#3, Tape \#2 \\
\hline $0: 1: 20$ & $\begin{array}{l}367.9 \mathrm{~m} \\
(1207 \mathrm{ft})\end{array}$ & $\begin{array}{l}\text { Top of fracture interval; high-angle fracture } \\
\text { evident by planar facets in borehole wall }\end{array}$ \\
\hline $0: 3: 20$ & $\begin{array}{l}374.6 \mathrm{~m} \\
(1229 \mathrm{ft})\end{array}$ & $\begin{array}{l}\text { Gyro reading; on left is N-S-trending } \\
\text { high-angle fracture; in upper right is } \\
\text { parabolic fracture trace }\end{array}$ \\
\hline $0: 3: 36$ & $\begin{array}{l}375.5 \mathrm{~m} \\
(1232.9)\end{array}$ & $\begin{array}{l}\text { Several red stains; fractures on } \\
\text { right side of screen }\end{array}$ \\
\hline $0: 4: 25$ & $\begin{array}{l}378.6 \mathrm{~m} \\
(1242 \mathrm{ft})\end{array}$ & Rough-textured borehole \\
\hline $0: 5: 18$ & $\begin{array}{l}381.3 \mathrm{~m} \\
(1251 \mathrm{ft})\end{array}$ & Breakout on right (SE) side \\
\hline $0: 6: 39$ & $\begin{array}{l}386.8 \mathrm{~m} \\
(1269 \mathrm{ft})\end{array}$ & $\begin{array}{l}\text { Near-vertical fracture on right side } \\
\text { of screen with SE-NW trend }\end{array}$ \\
\hline $0: 7: 43$ & $\begin{array}{l}388.3 \mathrm{~m} \\
(1274 \mathrm{ft})\end{array}$ & $\begin{array}{l}\text { Several fractures on upper right } \\
\text { screen }\end{array}$ \\
\hline $0: 8: 31$ & $\begin{array}{l}392.6 \mathrm{~m} \\
(1288 \mathrm{ft})\end{array}$ & $\begin{array}{l}\text { Light-colored band appears truncated } \\
\text { by light-colored parabolic trace }\end{array}$ \\
\hline $0: 8: 58$ & $\begin{array}{l}394.7 \mathrm{~m} \\
(1295 \mathrm{ft})\end{array}$ & $\begin{array}{l}\text { Two light-colored parabolic traces } \\
\text { with low to moderate dip }\end{array}$ \\
\hline $\begin{array}{l}0: 9: 13- \\
0: 10: 11\end{array}$ & $\begin{array}{l}395.9-400.2 \mathrm{~m} \\
(1299-1313 \mathrm{ft})\end{array}$ & $\begin{array}{l}\text { Several irregular parabolic traces within this } \\
\text { interval (low to moderate bedding dip) }\end{array}$ \\
\hline $0: 10: 27$ & $\begin{array}{l}401.4 \mathrm{~m} \\
(1317 \mathrm{ft})\end{array}$ & $\begin{array}{l}\text { Fracture sets trend E-W and dip N; } \\
\text { other fracture sets trend NE-SW }\end{array}$ \\
\hline $0: 11: 08$ & $\begin{array}{l}423.7 \mathrm{~m} \\
(1390 \mathrm{ft})\end{array}$ & Possible low-angle bedding \\
\hline $0: 11: 50$ & $\begin{array}{l}405.4 \mathrm{~m} \\
(1330 \mathrm{ft})\end{array}$ & $\begin{array}{l}\text { Light and dark color of borehole } \\
\text { possibly due to washout }\end{array}$ \\
\hline $0: 12: 45$ & $\begin{array}{l}410.6 \mathrm{~m} \\
(1347 \mathrm{ft})\end{array}$ & Two parabolic fracture traces \\
\hline
\end{tabular}


Table 17. Downhole Camera Video Log for ER-12-1 (Surface to $486.3 \mathrm{~m}$ (1596 ft) Interval, 08-21-91) (continued).

\begin{tabular}{|c|c|c|}
\hline Tape Time (Hr Min Sec) & Depth & Comments \\
\hline $0: 13: 18$ & $\begin{array}{l}413.5 \mathrm{~m} \\
(1356.6 \mathrm{ft})\end{array}$ & Parabolic fracture trace \\
\hline $0: 13: 35$ & $\begin{array}{l}414.8 \mathrm{~m} \\
(1361 \mathrm{ft})\end{array}$ & $\begin{array}{l}\text { Breakout on left due to fracture with SW-NE } \\
\text { trend; small influx of water at base }\end{array}$ \\
\hline $0: 16: 44$ & $\begin{array}{l}419.4 \mathrm{~m} \\
(1375.9 \mathrm{ft})\end{array}$ & Several more parabolic fracture traces \\
\hline $0: 17: 51$ & $\begin{array}{l}422.4 \mathrm{~m} \\
(1386 \mathrm{ft})\end{array}$ & Several fractures \\
\hline $0: 18: 56$ & $\begin{array}{l}427.0 \mathrm{~m} \\
(1401 \mathrm{ft})\end{array}$ & $\begin{array}{l}\text { Borehole surface has well-fractured } \\
\text { appearance }\end{array}$ \\
\hline $0: 20: 25$ & $\begin{array}{l}433.4 \mathrm{~m} \\
(1422 \mathrm{ft})\end{array}$ & $\begin{array}{l}\text { High-angle parabolic trace, reddish } \\
\text { in color }\end{array}$ \\
\hline $0: 22: 00$ & $\begin{array}{l}439.5 \mathrm{~m} \\
(1442 \mathrm{ft})\end{array}$ & Washout on right side \\
\hline $0: 22: 42$ & $\begin{array}{l}442.3 \mathrm{~m} \\
(1451 \mathrm{ft})\end{array}$ & $\begin{array}{l}\text { Light-colored calcite (?) veins on } \\
\text { right side of screen }\end{array}$ \\
\hline 0:24:07 & $\begin{array}{l}448.6 \mathrm{~m} \\
(1471.9 \mathrm{ft})\end{array}$ & $\begin{array}{l}\text { Fracture-controlled breakout on left } \\
\text { side of screen (NE-SW trend) }\end{array}$ \\
\hline $0: 24: 54$ & $\begin{array}{l}451.1 \mathrm{~m} \\
(1480 \mathrm{ft})\end{array}$ & Light-colored calcite (?) veins \\
\hline $0: 25: 23$ & $\begin{array}{l}453.5 \mathrm{~m} \\
(1488 \mathrm{ft})\end{array}$ & Rough washout on left \\
\hline 0:29:00 & $\begin{array}{l}467.0 \mathrm{~m} \\
(1532 \mathrm{ft})\end{array}$ & $\begin{array}{l}\text { Looking down at fluid level several } \\
\text { feet below }\end{array}$ \\
\hline $0: 30: 00$ & $\begin{array}{l}467.0 \mathrm{~m} \\
(1532 \mathrm{ft})\end{array}$ & Gyro drift check \\
\hline $0: 36: 54$ & $\mathrm{~N} / \mathrm{A}$ & $\begin{array}{l}\text { Change out ringlight to system with } \\
\text { light source below lens for observation } \\
\text { below water table }\end{array}$ \\
\hline \multicolumn{3}{|l|}{ Tape \#2; Run \#4 } \\
\hline $\mathrm{NA}$ & NA & Start of run \#4; black and white \\
\hline NA & $\begin{array}{l}467.1 \mathrm{~m} \\
(1532.5 \mathrm{ft})\end{array}$ & Looking down at water level \\
\hline NA & $\begin{array}{l}486.3 \mathrm{~m} \\
(1595.4 \mathrm{ft})\end{array}$ & $\begin{array}{l}\text { Deepest penetration below fluid level; } \\
\text { visibility is very poor to useless }\end{array}$ \\
\hline
\end{tabular}

Downhole camera contractor: Westech Engineering

Onsite engineer/operator: Greg Lianville

Date Run: 08-21-91

Commentator: Lance Protho, RSN 
Table 18. List of 35-mm Slides Documenting Drilling Activities, Equipment, and Samples at ER-12-1.* (Photography by RSN Geology/Hydrology Section)

\begin{tabular}{|c|c|c|}
\hline Slide No. & Date & Description \\
\hline ER0003 & $08-06-91$ & View from Dolomite Hill \\
\hline ER0004 & $08-06-91$ & Looking back up Dolomite Hill \\
\hline ER0005 & $08-12-91$ & Samples and sample log book on desk in sample skid shack \\
\hline ER0006 & $08-12-91$ & Locked sample box for "custody samples" at ER-12-1 \\
\hline ER0007 & $08-12-91$ & Locked sample box and previous locked-pipe sample holder \\
\hline ER0008 & $08-30-91$ & Cardwell 500 rig; pulling core barrel, Core \#1 \\
\hline ER0009 & $08-30-91$ & Unscrewing inner core barrel, Core \#1 \\
\hline ER0010 & $08-30-91$ & $\begin{array}{l}\text { Laying down fiberglass inner core barrel onto rig catwalk, } \\
\text { Core \#1 }\end{array}$ \\
\hline ER0011 & $08-30-91$ & Conventional core barrel, Core \#1 \\
\hline ER0012 & $08-30-91$ & Close-up of marine core bit \\
\hline ER0014 & $08-30-91$ & Marine core barrel and bit \\
\hline ER0016 & $08-30-91$ & $14 \mathrm{~cm}(5-1 / 2$ in) dual-string drill pipe used at ER-12-1 \\
\hline ER0018 & $08-30-91$ & Looking across reserve pit at Cardwell 500 while drilling \\
\hline ER0020 & $08-30-91$ & Radsafe and geologist trailer at ER-12-1 \\
\hline ER0021 & $08-30-91$ & Cardwell 500 rig \\
\hline ER0023 & $08-30-91$ & DRI van \\
\hline ER0024 & $08-30-91$ & Cardwell 500 rig from E-Tunnel access road \\
\hline ER0026 & $08-30-91$ & Cardwell 500 rig at distance from E-Tunnel access road \\
\hline ER0027 & $08-30-91$ & Core \#1 recovered $0.06 \mathrm{~m}(0.2 \mathrm{ft})$ of dolomite \\
\hline ER0029 & $08-30-91$ & Marine core barrel and bit on rig catwalk; used for Core \#2 \\
\hline ER0031 & $09-12-91$ & Rig geolograph (depth recorder) \\
\hline ER0033 & $09-12-91$ & Shale shaker and steel mud tank \\
\hline ER0035 & $09-12-91$ & Rotating head/well head assembly \\
\hline ER0037 & $09-12-91$ & Shaleshaker, top view \\
\hline ER0039 & $09-12-91$ & Steel mud tank \\
\hline ER0041 & $09-12-91$ & Rotary table \\
\hline ER0043 & $09-12-91$ & Driller's console rig controls and weight indicator \\
\hline ER0045 & $09-16-91$ & ER-12-1 location from side of hill 6355.0 , looking north \\
\hline ER0047 & $09-16-91$ & Area 12 camp from E-Tunnel access road, looking northeast \\
\hline ER0048 & $09-16-91$ & $\begin{array}{l}\text { ER-12-1 location from side of hill } 6355.0 \text {, looking north- } \\
\text { northeast }\end{array}$ \\
\hline ER0050 & $09-16-91$ & $\begin{array}{l}\text { ER-12-1 location from side of hill } 6355.0 \text {, looking north- } \\
\text { northwest }\end{array}$ \\
\hline ER0051 & $09-16-91$ & Cardwell 500 drill rig, truck-cab end \\
\hline ER0052 & $09-16-91$ & Tritium analyzer used at ER-12-1 \\
\hline ER0053 & $09-16-91$ & Tritium lab test equipment at ER-12-1 \\
\hline ER0054 & $09-16-91$ & Alpha detector at ER-12-1 \\
\hline ER0056 & $09-16-91$ & $\begin{array}{l}\text { Drilling fluid filtrate press and } \mathrm{N}_{2} \text { bottle arrangement used } \\
\text { at ER-12-1 }\end{array}$ \\
\hline ER0058 & $09-16-91$ & Radsafe and geologist trailer as equipped at ER-12-1 \\
\hline ER0060 & $09-16-91$ & $44.4 \mathrm{~cm}(17-1 / 2 \mathrm{in})$ button bit \\
\hline ER0062 & $09-16-91$ & $31.1 \mathrm{~cm}(12-1 / 4 \mathrm{in})$ button bit used at ER-12-1 \\
\hline
\end{tabular}


Table 18. List of 35-mm Slides Documenting Drilling Activities, Equipment, and Samples at ER-12-1.* (Photography by RSN Geology/Hydrology Section) (continued).

\begin{tabular}{|c|c|c|}
\hline Slide No. & Date & Description \\
\hline ER0064 & $10-08-91$ & "Full-hole" drill pipe on pipe racks at ER-12-1 \\
\hline ER0065 & $10-08-91$ & Derrick full of pipe after Core \#4 \\
\hline ER0067 & $10-08-91$ & $22.2 \mathrm{~cm}(8-3 / 4 \mathrm{in})$ diamond core bit used for Core \#4 \\
\hline ER0069 & $10-08-91$ & $\begin{array}{l}\text { Using rock saw to cut fiberglass inner barrel with Core \#4 } \\
\text { inside }\end{array}$ \\
\hline ER0071 & $10-08-91$ & Examining Core \#4 in core trough \\
\hline ER0073 & $10-08-91$ & Core barrel laying in V-door ramp after Core \#4 \\
\hline ER0075 & $10-08-91$ & Geologist examining Core \#4 \\
\hline ER0078 & $10-08-91$ & Core \#4, Box \#7; $902.3-903.2 \mathrm{~m}(2959.5-2962.5 \mathrm{ft})$ \\
\hline ER0080 & $10-08-91$ & Core \#4, Box \#6; 900.5 - $902.3 \mathrm{~m}(2953.8$ - $2959.5 \mathrm{ft})$ \\
\hline ER0082 & $10-08-91$ & Core \#4, Box \#5; $898.9-900.5 \mathrm{~m}(2948.5-2953.8 \mathrm{ft})$ \\
\hline ER0084 & $10-08-91$ & Core \#4, Box \#4; 897.5 - 898.9 m (2943.7 - $2948.5 \mathrm{ft})$ \\
\hline ER0086 & $10-08-91$ & Core \#4, Box \#3; $895.4-897.5$ m $(2937.0-2943.7 \mathrm{ft})$ \\
\hline ER0088 & $10-19-91$ & AWS logging sheave at ER-12-1; lower sheave at rig floor \\
\hline ER0090 & $10-19-91$ & $31.1 \mathrm{~cm}(12-1 / 4)$ in button bit used at ER-12-1 \\
\hline ER0092 & $10-19-91$ & AWS flat bed truck with logging tools at ER-12-1 \\
\hline ER0094 & $10-19-91$ & View of AWS logging truck from ER-12-1 rig floor \\
\hline ER0096 & $10-19-91$ & AWS logging truck at ER-12-1, side view \\
\hline ER0098 & $10-19-91$ & $\begin{array}{l}\text { AWS logging truck at ER-12-1, front view with rig in } \\
\text { background }\end{array}$ \\
\hline ER0100 & $10-19-91$ & $\begin{array}{l}\text { AWS pickup truck with logging tools and lead pig for storing } \\
\text { radioactive logging sources }\end{array}$ \\
\hline ER0102 & $10-19-91$ & AWS logging sheave attached to traveling blocks at ER-12-1 \\
\hline ER0104 & $10-19-91$ & AWS instrument-control trailer for VSP surveys at ER-12-1 \\
\hline ER0106 & $10-19-91$ & Calibration box for ENP logging tool \\
\hline ER0108 & $10-19-91$ & $\begin{array}{l}\text { Inside of VSP instrument trailer, Steve Molnar operator at } \\
\text { ER-12-1 }\end{array}$ \\
\hline ER0110 & $10-19-91$ & AWS VSP truck at ER-12-1 \\
\hline ER0112 & $10-19-91$ & AWS VSP truck, seismic-source pad \\
\hline ER0114 & $10-19-9 \mid$ & Compressor and motor instruments at back of VSP truck \\
\hline ER0116 & $10-19-91$ & Air filters used while drilling with air at ER-12-1 \\
\hline ER0117 & $10-19-91$ & Betaman tritium instrument at ER-12-1 \\
\hline ER0118 & $10-19-91$ & Bromide measuring instrument at ER-12-1 \\
\hline ER0120 & $10-19-91$ & Turbidity meter at ER-12-1 \\
\hline ER0121 & $12-05-91$ & Intergraph CADD work station in the RSN Geology Building \\
\hline ER0122 & $12-05-91$ & $\begin{array}{l}\text { Geologic work station in the "GCP Room" at the USGS Core } \\
\text { Library }\end{array}$ \\
\hline ER0124 & $12-05-91$ & $\begin{array}{l}\text { Closeup of microscope work station in the "GCP Room" at } \\
\text { the USGS Core Library }\end{array}$ \\
\hline ER0126 & $\begin{array}{l}12-05-91 \\
\text { at the USGS }\end{array}$ & $\begin{array}{l}\text { ER-12-1 "paleo" samples on drying tray in the "GCP Room" } \\
\text { Core Library }\end{array}$ \\
\hline ER0128 & $12-05-91$ & $\begin{array}{l}\text { ER-12-1 boxed drill cuttings on sample cart in the "GCP } \\
\text { Room" at the USGS Core Library }\end{array}$ \\
\hline
\end{tabular}


Table 18. List of 35-mm Slides Documenting Drilling Activities, Equipment, and Samples at ER-12-1.* (Photography by RSN Geology/Hydrology Section) (continued).

\begin{tabular}{|c|c|c|}
\hline Slide No. & Date & Description \\
\hline ER0130 & $12-05-91$ & $\begin{array}{l}\text { Work area (sink \& counter top) in the "GCP Room" at the } \\
\text { USGS Core Library }\end{array}$ \\
\hline ER0131 & $12-05-91$ & ER-12-1 washed drill cuttings, $3.0-64.0 \mathrm{~m}(10-210 \mathrm{ft})$ interval \\
\hline ER0133 & $12-05-91$ & ER-12-1 washed drill cuttings, $67.1-128.0 \mathrm{~m}(220-420 \mathrm{ft})$ interval \\
\hline ER0135 & $12-05-91$ & 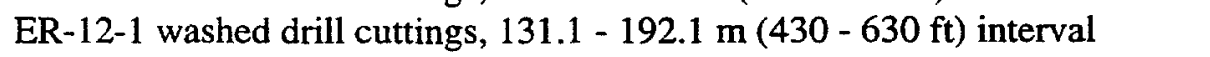 \\
\hline ER0137 & $12-05-91$ & ER-12-1 washed drill cuttings, $195.1-259.1 \mathrm{~m}(640-850 \mathrm{ft})$ interval \\
\hline ER0139 & $12-05-91$ & ER-12-1 washed drill cuttings, $262.2-323.2 \mathrm{~m}(860-1060 \mathrm{ft})$ interval \\
\hline ER0141 & $12-05-91$ & ER-12-1 washed drill cuttings, $326.2-387.2 \mathrm{~m}(1070-1270 \mathrm{ft})$ interval \\
\hline ER0143 & $12-05-91$ & ER-12-1 washed drill cuttings, $390.2-451.2 \mathrm{~m}(1280-1480 \mathrm{ft})$ interval \\
\hline ER0145 & $12-05-91$ & ER-12-1 washed drill cuttings, $454.3-515.3 \mathrm{~m}(1490-1690 \mathrm{ft})$ interval \\
\hline ER0147 & $12-05-91$ & ER-12-1 washed drill cuttings, $518.3-585.4 \mathrm{~m}(1700-1920 \mathrm{ft})$ interval \\
\hline ER0149 & $12-05-91$ & ER-12-1 washed drill cuttings, $588.4-649.4 \mathrm{~m}(1930-2130 \mathrm{ft})$ interval \\
\hline ER0151 & $12-05-91$ & ER-12-1 washed drill cuttings, $652.4-713.4 \mathrm{~m}(2140-2340 \mathrm{ft})$ interval \\
\hline ER0153 & $12-05-91$ & ER-12-1 washed drill cuttings, $716.5-777.4 \mathrm{~m}(2350-2550 \mathrm{ft})$ interval \\
\hline ER0155 & $12-05-91$ & ER-12-1 washed drill cuttings, $780.5-841.5 \mathrm{~m}(2560-2760 \mathrm{ft})$ interval \\
\hline ER0157 & $12-05-91$ & ER-12-1 washed drill cuttings, $844.5-905.5 \mathrm{~m}(2770-2970 \mathrm{ft})$ interval \\
\hline ER0159 & $12-05-91$ & ER-12-1 washed drill cuttings, $908.5-972.6 \mathrm{~m}(2980-3190 \mathrm{ft})$ interval \\
\hline ER0161 & $12-05-91$ & ER-12-1 washed drill cuttings, $975.6-1033.5 \mathrm{~m}(3200-3390 \mathrm{ft})$ interval \\
\hline ER0163 & $12-05-91$ & ER-12-1 washed drill cuttings, $1036.6-1081.7 \mathrm{~m}(3400-3584 \mathrm{ft})$ interval \\
\hline ER0165 & $12-05-91$ & ER-12-1 washed drill cuttings with custody seal \\
\hline ER0167 & $12-05-91$ & ER-12-1 Core Run \#3 with Formation Micro-Scanner log \\
\hline ER0168 & $12-05-91$ & ER-12-1 Core Run \#3, Box \#1, $751.2-753.2 \mathrm{~m}(2464-2470.7 \mathrm{ft})$ interval \\
\hline ER0170 & $12-05-91$ & ER-12-1 Core Run \#3, Box \#2,753.2 - $754.3 \mathrm{~m}(2470.7$ - $2474 \mathrm{ft})$ interval \\
\hline ER0172 & $12-05-91$ & ER-12-1 Core Run \#1, Box \#2, $539.3-543.9 \mathrm{~m}(1769-1784 \mathrm{ft})$ interval \\
\hline ER0174 & $12-05-91$ & ER-12-1 Core Run \#4, Box \#3, 895.4 - $897.5 \mathrm{~m}(2937$ - $2943.7 \mathrm{ft})$ interval \\
\hline ER0175 & $12-05-91$ & ER-12-1 Core Run \#4, Box \#4, 897.5 - $898.9 \mathrm{~m}(2943.7$ - 2948.5) ft interval \\
\hline ER0176 & $12-05-91$ & ER-12-1 Core Run \#4, Box \#5, 898.9 - $900.5 \mathrm{~m}$ (2948.5 - 2953.8) ft interval \\
\hline ER0177 & $12-05-91$ & ER-12-1 Core Run \#4, Box \#6, 900.5 - $902.3 \mathrm{~m}(2953.8$ - 2959.5) ft interval \\
\hline ER0178 & $12-05-91$ & ER-12-1 Core Run \#4, Box \#7, 902.7 - $903.2 \mathrm{~m}(2959.5$ - 2962.5) ft interval \\
\hline ER0179 & $12-05-91$ & $\begin{array}{l}\text { ER-12-1 Core Run \#4, close up of macrofossil fragments at } \\
897.8-898.4 \mathrm{~m}(2945 \text { and } 2947 \mathrm{ft})\end{array}$ \\
\hline ER0180 & $12-05-91$ & $\begin{array}{l}\text { ER-12-1 Core Run \#4, close up of macrofossil fragments at } \\
897 \mathrm{~m}(2942 \mathrm{ft})\end{array}$ \\
\hline ER0181 & $12-05-91$ & $\begin{array}{l}\text { Intergraph CADD work station in the RSN Geology building, } \\
\text { Mercury }\end{array}$ \\
\hline
\end{tabular}

*A set of color photos were taken by a LLNL staff photographer at the direction of "D" Donithan, DRI, of the ER-12-1 location and selected equipment. All slides in possession of DOE-ERD, Las Vegas, NV. 
Table 19. Stratigraphic Nomenclature used in this report for the pre-Tertiary rocks at the NTS. (Formerly used nomenclature for roughly equivalent units shown parenthetically.)

\begin{tabular}{|c|c|c|c|}
\hline Ki..... & Cretaceous Intrusive (dike) & Oe $\ldots$ & Eureka Quartzite \\
\hline $\mathrm{Kg}$. & Cretaceous Plutonic Rocks & $\mathrm{Oa} \ldots$ & Antelope Valley Limestone \\
\hline Kgg . & Gold Meadows Stock & On .... & Ninemile Formation \\
\hline Kgc . & Climax Stock & $\mathrm{Og}$ & Goodwin Formation \\
\hline Kgt. & Twinridge Stock & $\mathrm{Cn}$ & Nopah Formation \\
\hline PPbs. & $\begin{array}{l}\text { Bird Spring Formation } \\
\text { (Tippipah Limestone) }\end{array}$ & Cnd & Dunderberg Shale \\
\hline $\mathrm{MDe} \ldots$ & Eleana Formation & $\mathrm{Cb}$ & Bonanza King Formation \\
\hline Dg. & $\begin{array}{l}\text { Guilmette Formation } \\
\text { (Devils Gate Limestone) }\end{array}$ & $\mathrm{Cc} \ldots$ & Carrara Formation \\
\hline Ds & $\begin{array}{l}\text { Simonson Dolomite } \\
\text { (Nevada Formation) }\end{array}$ & $\mathrm{Cz}$. & Zabriskie Quartzite \\
\hline DSs & Sevy Dolomite & $\mathrm{CZw}$ & Wood Canyon Formation \\
\hline Sl & $\begin{array}{l}\text { Laketown Dolomite } \\
\text { (Lone Mountain Dolomite) }\end{array}$ & Zs & Stirling Quartzite \\
\hline Oes & Ely Springs Dolomite & $\mathrm{Zj} \ldots$ & Johnnie Formation \\
\hline
\end{tabular}

Mississippian Eleana Formation. Another section of (lower) Simonson Dolomite occurs from 874.2

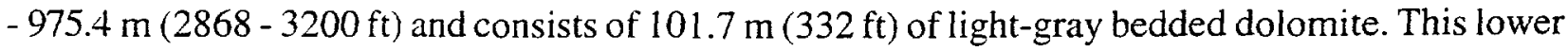
Simonson Dolomite conformably overlies the upper Sevy Dolomite at 975.4 - 1086.3 m (3200 $3564 \mathrm{ft})$.

A sliver of Antelope Valley Limestone (middle Ordovician) at $237.7-274.3 \mathrm{~m}$ (780 - $900 \mathrm{ft}$ ) lies in fault contact between two intervals of Sevy Dolomite (late Silurian). This fractured, varicolored dolomite and dolomite breccia would not have been identified as Antelope Valley Limestone if it were not for its middle Ordovician conodont assemblage (A.G. Harris, USGS-Reston, VA).

Two intervals of the Mississippian Eleana Formation were penetrated at ER-12-1. The assignments of these rocks to Unit $J$ of the Eleana is chiefly based on the uniformly fine-grained nature of both sections encountered in ER-12-1 and to the fact that Unit $J$ defined by Poole and others (1961) consists of several thousand feet of siltstone, shale, and argillite. However, it should be noted 
Table 20. Stratigraphic Log for ER-12-1 (November 1991).

\begin{tabular}{|c|c|c|c|c|c|c|c|}
\hline \multicolumn{3}{|c|}{ Depth } & \multirow[b]{2}{*}{ Lithology } & \multirow[b]{2}{*}{ Formation } & \multirow[b]{2}{*}{ Symbol } & \multicolumn{2}{|c|}{ Thickness } \\
\hline & Meters & Feet & & & & Meters & Feet \\
\hline 0 & -7.9 & $0-26$ & Alluvium & Alluvium & QTa & 7.9 & 26 \\
\hline 7.9 & -237.8 & $26-780$ & Dolomite & Sevy Dolomite & DSs & 230.0 & 754 \\
\hline 237.8 & -240.8 & $780-790$ & Dolomite Breccia & Fault Zone & - & $\sim 3.0$ & $\sim 10$ \\
\hline 240.8 & -274.3 & $790-900$ & Dolomite Breccia & Antelope Valley Limestone & $\mathrm{Oa}$ & 33.5 & 110 \\
\hline 274.3 & -312.4 & $900-1025$ & Dolomite & Sevy Dolomite & DSs & 38.1 & 125 \\
\hline 312.4 & -521.2 & $1025-1710$ & $\begin{array}{l}\text { Interbedded calcareous, } \\
\text { siltstone, siliceous siltstone } \\
\text { and shale }\end{array}$ & $\begin{array}{l}\text { Eleana Formation, } \\
\text { Unit J (?) }\end{array}$ & MDej & 208.8 & 685 \\
\hline 521.2 & -550.8 & $1710-1807$ & Dolomite & $\begin{array}{l}\text { Upper Simonson Dolomite } \\
\text { or Lower Guilmette Formati }\end{array}$ & $\begin{array}{l}\text { Ds - Dg } \\
\text { ion }\end{array}$ & 29.6 & 97 \\
\hline 550.8 & -856.8 & $1807-2811$ & $\begin{array}{l}\text { Interbedded siltstone, } \\
\text { Chert sandstone, and shale }\end{array}$ & $\begin{array}{l}\text { Eleana Formation, } \\
\text { Unit J (?) }\end{array}$ & MDej & 306.0 & 1004 \\
\hline 856.8 & -874.2 & $2811-2868$ & Calcareous siltstone & $\begin{array}{l}\text { Eleana Formation, } \\
\text { Unit J (?) }\end{array}$ & MDej & 17.4 & 57 \\
\hline 874.2 & -975.4 & $2868-3200$ & Dolomite & Lower Simonson Dolomite & Ds & 101.2 & 332 \\
\hline 975.4 & -1086.3 & $3200-3564$ & Dolomite & Upper Sevy Dolomite & DSs & 110.9 & 364 \\
\hline 1086.3 & -1093.6 & $3564-3588$ & Biotite Lamprophyre & Intrusive & $\mathrm{n} / \mathrm{a}$ & $>7.3$ & $>24$ \\
\hline
\end{tabular}


Table 21. Lithologic Log for ER-12-1. (Logged by S. L. Drellack and L. B. Prothro, RSN, 11/91)

Unless otherwise noted, the following descriptions refer to washed cuttings samples at $3.05 \mathrm{~m}(10 \mathrm{ft})$ intervals. Colors are determined by comparing wet sample color to the Geological Society of America Rock-Color Chart. Stratigraphic contacts and lithologic divisions are tied to geophysical logs whenever possible. Note: Several new stratigraphic assignments, based on recent conodont age brackets (A.G. Harris, USGS - Reston, VA) have been applied to the original 11/91 lithologic log. Inclusion of XRD and micropaleontological analyses have modified original lithologic description.

\begin{tabular}{|c|c|c|}
\hline Depth & Lithologic Description & Stratigraphic Unit \\
\hline
\end{tabular}

$0-6.1 \mathrm{~m}$

$(0-20 \mathrm{ft})$

$6.1-7.9 \mathrm{~m}$

$(20-26 \mathrm{ft})$

$7.9-15.2 \mathrm{~m}$

$(26-50 \mathrm{ft})$

$15.2-36.6 \mathrm{~m}$

$(50-120 \mathrm{ft})$

$36.6-183.5 \mathrm{~m}$

$(120-602 \mathrm{ft})$
Alluvium consisting of $90 \%$ tuffaceous

fragments and $10 \%$ carbonate fragments.

Tuffaceous fragments are zeolitic, devitrified, and range in color from grayish-yellow (zeolitic fragments) to pale-reddish-brown (devitrified fragments). Carbonate fragments consist of predominantly grayish-black, finely crystalline dolomite.

Alluvium consisting of $90 \%$ carbonate fragments and $10 \%$ tuffaceous fragments. Carbonate fragments consist of yellowish-gray and grayish-black, finely to cryptocrystalline dolomite. Tuffaceous fragments are similar to interval $0-6.1 \mathrm{~m}(0-20 \mathrm{ft})$.

Dolomite: Dusky-yellowish-brown;

well indurated; finely crystalline;no visible porosity; weak fetid odor when broken. Moderatebrown, very fine-grained, moderately indurated dolomitic sandstone at approximately $12.2 \mathrm{~m}(40 \mathrm{ft})$.

Dolomite: Grayish-red (10 R 4/2); moderately to well indurated; finely crystalline; saccharoidal; sandy in parts, more apparent after etching in HCL; slightly argillaceous; no visible porosity; weak fetid odor when broken.

Dolomite: Varicolored, but mostly olive-gray and medium-dark-gray to black, some mottled with dusky-red; well indurated; finely crystalline to cryptocrystalline; dense; veinlets of white dolomite, generally less than $1 \mathrm{~mm}$ thick, occur at various depths throughout interval; trace dusky-red and dark yellowish-orange staining associated with veinlets; no visible porosity; darker color dolomite yields weak fetid odor when broken. Color changes imply bedding on the order of a few meters thick. The $88.4 \mathrm{~m}(290 \mathrm{ft})$ sample shows an increase in dusky-red staining and several pieces of recemented breccia with some vuggy and interfragment porosity. An increase in dusky-red and dark-yellowish-orange stain in the $106.7 \mathrm{~m}(350 \mathrm{ft})$ sample may correspond to an increase in uranium on the Spectralog at $104.5 \mathrm{~m}(343 \mathrm{ft})$. Fractures were also observed at this depth on the downhole camera.
Alluvium

Alluvium

Lower Sevy ${ }^{1}$

Dolomite

\section{Lower Sevy ${ }^{1}$}

Dolomite

\section{Lower Sevy 1}

Dolomite 
Table 21. Lithologic Log for ER-12-1. (Logged by S. L. Drellack and L. B. Prothro, RSN, 11/91) (continued).

\begin{tabular}{|c|c|c|}
\hline Depth & Lithologic Description & Stratigraphic Unit \\
\hline $\begin{array}{l}183.5-237.7 \mathrm{~m} \\
(602-780 \mathrm{ft})\end{array}$ & $\begin{array}{l}\text { Dolomite: Varicolored, medium-light- } \\
\text { gray to medium-dark-gray, dark-olive-gray, } \\
\text { and olive-gray, but with an overall lighter } \\
\text { appearance than overlying interval; well } \\
\text { indurated; medium to finely crystalline; sandy } \\
\text { and silty; moderate-red ( } 5 \mathrm{R} 5 / 4) \text { and trace of } \\
\text { dark-yellowish-orange iron oxide staining at } \\
\text { various depths throughout interval, but } \\
\text { particularly from approximately } \\
185.9 \text { - } 192.0 \mathrm{~m}(610-630 \mathrm{ft}) \text {. }\end{array}$ & $\begin{array}{l}\text { Lower Sevy } \\
\text { Dolomite }\end{array}$ \\
\hline $\begin{array}{l}237.7-259.1 \mathrm{~m} \\
(780-850 \mathrm{ft})\end{array}$ & $\begin{array}{l}\text { Dolomite: Various shades of red and } \\
\text { yellow (color is the result of pre- } \\
\text { valent iron oxide staining); well indurated; } \\
\text { coarsely to finely crystalline; sandy and silty } \\
\text { in part (more apparent after etching in } \\
\text { HCL); some fragments appear intensely } \\
\text { fractured; no visible porosity. Also, } \\
\text { common pale- to dark-yellowish-orange, } \\
\text { moderately indurated fault breccia fragments. } \\
\text { Faulted lower contact. }\end{array}$ & $\begin{array}{l}\text { Antelope Valley } \\
\text { Limestone }^{1} \text {, Aysees } \\
\text { Peak member and } \\
\text { fault breccia }\end{array}$ \\
\hline $\begin{array}{l}259.1-274.3 \mathrm{~m} \\
(850-900 \mathrm{ft})\end{array}$ & $\begin{array}{l}\text { Dolomite: Light-gray to medium-light- } \\
\text { gray and mottled, becoming medium- } \\
\text { dark-gray below } 268.2 \mathrm{~m}(880 \mathrm{ft}) \text {; well } \\
\text { indurated; coarsely crystalline, some finely } \\
\text { to medium crystalline; appears intensely } \\
\text { fractured; common pale- to dark-yellowish- } \\
\text { orange iron oxide staining; no visible } \\
\text { porosity; moderate fetid odor when broken. } \\
\text { Also, minor pale- to dark-yellowish-orange, } \\
\text { pooriy to moderately indurated fault breccia } \\
\text { fragments. Slickensides on fault breccia } \\
\text { fragment noted in the } 271.3 \mathrm{~m}(890 \mathrm{ft}) \text { sample. } \\
\text { Faulted upper contact. }\end{array}$ & $\begin{array}{l}\text { Antelope Valley } \\
\text { Limestone } \\
\text { Aysees Peak member }\end{array}$ \\
\hline $\begin{array}{l}274.3-289.2 \mathrm{~m} \\
(900-949 \mathrm{ft})\end{array}$ & $\begin{array}{l}\text { Dolomite: Dark-gray to grayish-black; } \\
\text { well indurated; medium crystalline: no } \\
\text { visible porosity; weak fetid odor when } \\
\text { broken. Spectralog and downhole camera } \\
\text { indicate possible fault and/or fracture }(\mathrm{s}) \\
\text { at approximately } 285.3 \mathrm{~m}(936 \mathrm{ft}) \text {. }\end{array}$ & $\begin{array}{l}\text { Lower Sevy } \\
\text { Dolomite }\end{array}$ \\
\hline $\begin{array}{l}289.2-312.4 \mathrm{~m} \\
(949-1025 \mathrm{ft})\end{array}$ & $\begin{array}{l}\text { Dolomite: Light-brownish-gray to } \\
\text { medium-gray; well indurated; medium crystalline; } \\
\text { no visible porosity. Faulted lower contact. }\end{array}$ & $\begin{array}{l}\text { Lower Sevy } \\
\text { Dolomite }\end{array}$ \\
\hline
\end{tabular}


Table 21. Lithologic Log for ER-12-1. (Logged by S. L. Drellack and L. B. Prothro, RSN, 11/91) (continued).

\begin{tabular}{|c|c|c|}
\hline Depth & Lithologic Description & Stratigraphic Unit \\
\hline $\begin{array}{l}312.4-322.5 \mathrm{~m} \\
(1025-1058 \mathrm{ft})\end{array}$ & $\begin{array}{l}\text { Grayish calcareous siltstone; well indur- } \\
\text { ated; finely crystalline; argillaceous } \\
\text { (becoming increasingly more argillaceous } \\
\text { towards base); silty; laminated (more } \\
\text { apparent after etching in HCL); } \\
\text { moderate-red ( } 5 \mathrm{R} 4 / 6) \text { and dark-yellowish- } \\
\text { orange iron oxide coatings on some fragments; } \\
\text { no visible porosity. Trace calcite fracture } \\
\text { fill. Stained slickensides on fragment in } \\
320.0 \mathrm{~m}(1050 \mathrm{ft}) \text { sample. Faulted upper contact. }\end{array}$ & $\begin{array}{l}\text { Eleana Formation } 1 \text {, } \\
\text { Unit J (?) }\end{array}$ \\
\hline $\begin{array}{l}322.5-348.7 \mathrm{~m} \\
(1058-1144 \mathrm{ft})\end{array}$ & $\begin{array}{l}\text { Siltstone with minor interbedded } \\
\text { and interlaminated limestone. } \\
\text { Siltstone is grayish-black to black; } \\
\text { moderately to well indurated; argillaceous; } \\
\text { pyritic; weakly calcareous; laminated; } \\
\text { exhibits a bright sheen on partings; } \\
\text { fragments cut by numerous white calcite } \\
\text { veinlets; common grayish-orange to } \\
\text { dark-yellowish-orange iron oxide staining } \\
\text { from approximately } 326.1 \text { - } 341.4 \mathrm{~m} \\
\text { (1070 - } 1120 \mathrm{ft} \text { ). Minor interlaminated, } \\
\text { medium-dark-gray, argillaceous, finely } \\
\text { crystalline calcareous siltstone; more common } \\
\text { in upper } 9.1 \mathrm{~m} \text { ( } 30 \mathrm{ft} \text { ). Thin interbeds of } \\
\text { grayish-black, argillaceous, finely crystalline } \\
\text { calcareous siltstone below } 338.3 \mathrm{~m} \text { (1110 ft). } \\
\text { Numerous fragments of fault breccia are present } \\
\text { in sample at } 326.1 \mathrm{~m} \text { ( } 1070 \mathrm{ft} \text { ). }\end{array}$ & $\begin{array}{l}\text { Eleana Formation }{ }^{1} \text {, } \\
\text { Unit } \mathrm{J}(?)\end{array}$ \\
\hline $\begin{array}{l}348.7-368.8 \mathrm{~m} \\
(1144-1210 \mathrm{ft})\end{array}$ & $\begin{array}{l}\text { Shale with interbedded and interlaminated } \\
\text { limestone. Shale is grayish-black, } \\
\text { moderately indurated, silty, pyritic, may } \\
\text { grade into siltstone in places, breaks into } \\
\text { thin platy fragments that exhibit a bright } \\
\text { sheen on surfaces of partings, common lenses } \\
\text { and veinlets of white calcite. Calcareous siltstone } \\
\text { is similar to interval } 312.4-322.5 \mathrm{~m} \\
\text { (1025- } 1058 \mathrm{ft}) \text {. Dark-yellowish-orange and } \\
\text { light-brown }(5 \mathrm{YR} 5 / 6) \text { iron oxide staining below } \\
\text { approximately } 374.9 \mathrm{~m}(1230 \mathrm{ft}) \text {. }\end{array}$ & $\begin{array}{l}\text { Eleana Formation }{ }^{1} \text {, } \\
\text { Unit } J(?)\end{array}$ \\
\hline $\begin{array}{l}368.8-400.2 \mathrm{~m} \\
(1210-1313 \mathrm{ft})\end{array}$ & $\begin{array}{l}\text { Calcareous siltstone with interbedded and inter- } \\
\text { laminated shale. Calcareous siltstone is grayish- } \\
\text { black, finely crystalline, dense, argillaceous. } \\
\text { Shale is similar to interval above. }\end{array}$ & $\begin{array}{l}\text { Eleana Formation }{ }^{1} \text {, } \\
\text { Unit J (?) }\end{array}$ \\
\hline
\end{tabular}


Table 21. Lithologic Log for ER-12-1. (Logged by S. L. Drellack and L. B. Prothro, RSN, 11/91) (continued).

\begin{tabular}{|c|c|c|}
\hline Depth & Lithologic Description & Stratigraphic Unit \\
\hline $\begin{array}{l}400.2-521.2 \mathrm{~m} \\
(1313-1710 \mathrm{ft})\end{array}$ & $\begin{array}{l}\text { Siltstone and calcareous siltstone } \\
\text { interbeds with minor interbedded shale. } \\
\text { Siltstone is grayish-black to black, well indurated, } \\
\text { argillaceous, pyritic, laminated, and exhibits } \\
\text { a bright sheen on partings. Light-brown } \\
\text { ( } 5 \text { YR } 5 / 6 \text { ) to dark-yellowish-orange staining } \\
\text { common particularly in sample at } 472.4 \mathrm{~m} \\
\text { (1550 ft). Pyrite is finely disseminated but also } \\
\text { occurs as individual striated cubes up to } 1 / 2 \mathrm{~mm} \\
\text { in size. Calcareous siltstone is medium-dark-gray } \\
\text { to dark-gray, well indurated, finely to } \\
\text { cryptocrystalline, silty and argillaceous, slightly } \\
\text { pyritic, laminated in part, and randomly cut by } \\
\text { veinlets of white calcite, many of which } \\
\text { have been stained dark-yellowish-orange. } \\
\text { Cuttings indicate that the } 454.2-463.3 \mathrm{~m} \\
\text { (1490 - } 1520 \mathrm{ft} \text { ) interval is predominately } \\
\text { calcareous siltstone. Shale occurs as interbeds and } \\
\text { laminae and is black, moderately indurated, pyritic, } \\
\text { and weakly fissile. An increase in argillaceous } \\
\text { siltstone with very thin shaley laminae in } \\
487.7-521.2 \mathrm{~m} \text { ( } 1600 \text { - } 1700 \mathrm{ft} \text { ) interval. XRD } \\
\text { data for the } 463.3 \mathrm{~m} \text { ( } 1520 \mathrm{ft} \text { ) sample is given in } \\
\text { Table } 15 . \text { Faulted lower contact. }\end{array}$ & $\begin{array}{l}\text { Eleana Formation } 1 \\
\text { Unit J (?) }\end{array}$ \\
\hline $\begin{array}{l}521.2-550.8 \mathrm{~m} \\
(1710-1807 \mathrm{ft})\end{array}$ & 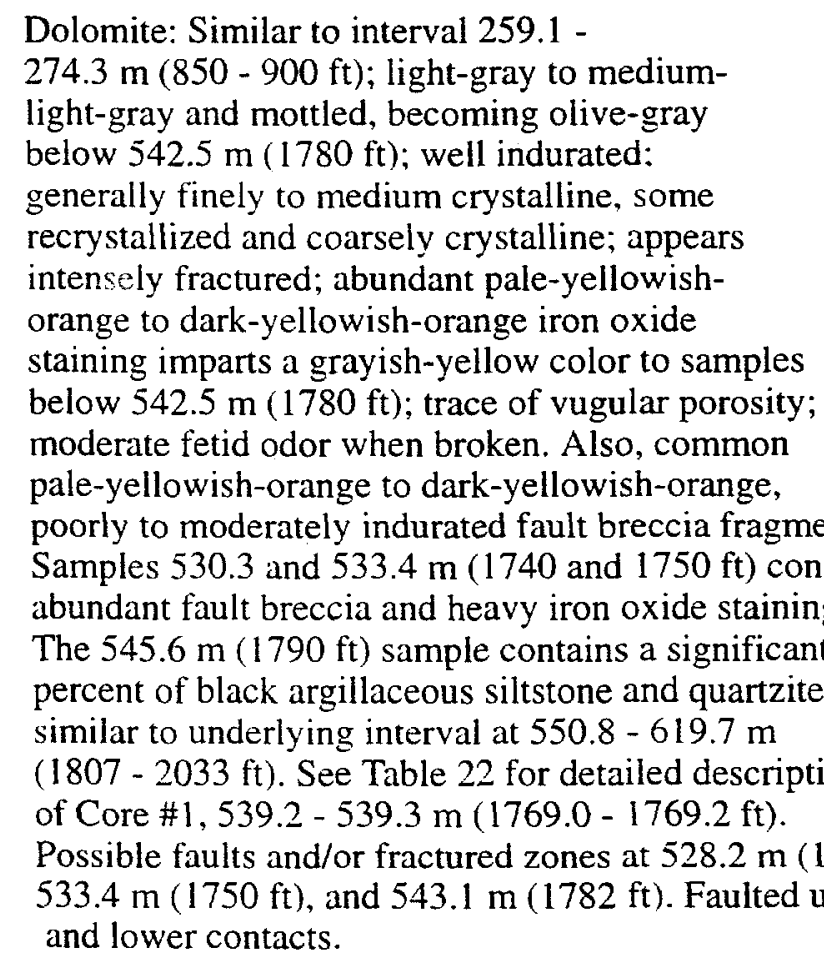 & $\begin{array}{l}. \\
\text { s. } \\
\text { in } \\
33 \mathrm{ft} \text { ), } \\
\end{array}$ \\
\hline
\end{tabular}


Table 21. Lithologic Log for ER-12-1. (Logged by S. L. Drellack and L. B. Prothro, RSN, 11/91) (continued).

\begin{tabular}{ll}
\hline Depth & Lithologic Description \\
\hline
\end{tabular}

$550.8-619.7 \mathrm{~m}$

$(1807-2033 \mathrm{ft})$

$619.7-657.1 \mathrm{~m}$

$(2033-2156 \mathrm{ft})$

$657.1-686.7 \mathrm{~m}$

$(2156-2253 \mathrm{ft})$

$686.7-712.3 \mathrm{~m}$

$(2253-2337 \mathrm{ft})$
Chert sandstone and minor Siltstone.

Chert sandstone is medium-dark-gray to

dark-gray, very well indurated and dense, and consists of fine- to medium-grained, subrounded, and moderately sorted clear quartz grains, scarce feldspar, and medium-light-gray to white chert. Chert fragments are often the largest grains (up to $1 \mathrm{~mm}$ ) in size. The chert sandstone is also argillaceous containing scarce to common clasts of black shale, pyritic, and very weakly calcareous and/or weakly dolomitic. Very scarce very-light-gray silica/chert veins. Siltstone is dark-gray, moderately indurated, argillaceous, pyritic, and occurs mostly as platy fragments with a bright sheen on surfaces of partings. Sample $560.8 \mathrm{~m}(1840 \mathrm{ft})$ is heavily contaminated with tuffaceous fragments. These fragments appear to be zeolitized tuffs typical of southern Yucca Flat and were incorporated into the drilling fluid from the mud system (steel mud pit, shale shaker, and mud pump). Spectralog indicates possible fault and/or fracture(s) at $591.3 \mathrm{~m}(1940 \mathrm{ft})$. Faulted upper contact.

Shale with interbedded chert sandstone and siltstone. Shale is black, moderately indurated, fissile, with a waxy texture on partings. The other lithologies are similar to the overlying interval with the exception of the chert sandstone, which is mostly fine-grained and is randomly cut by veinlets of white silica.

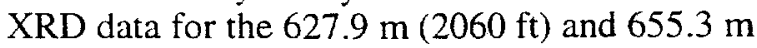
(2150 ft) samples are given in Table 15.

Shale: Lithology similar to shale in above interval. Trace argillaceous siltstone. Scarce fragments containing rare scattered clear quartz grains "floating" in shale matrix.

Interbedded chert sandstone, siltstone, and shale. Lithologies are similar to above intervals.
Eleana Formation, Unit J (?)

Eleana Formation, Unit J (?)

Eleana Formation, Unit J (?)

Eleana Formation, Unit J (?) 
Table 21. Lithologic Log for ER-12-1. (Logged by S. L. Drellack and L. B. Prothro, RSN, 11/91) (continued).

\begin{tabular}{|c|c|c|}
\hline Depth & Lithologic Description & Stratigraphic Unit \\
\hline $\begin{array}{l}712.3-760.2 \mathrm{~m} \\
(2337-2494 \mathrm{ft})\end{array}$ & $\begin{array}{l}\text { Chert sandstone and siltstone. Chert sandstone is } \\
\text { massive from } 714.1 \text { to } 723.6 \mathrm{~m}(2343- \\
2374 \mathrm{ft}) \text { and from } 749.8 \text { to } 760.2 \mathrm{~m}(2460- \\
2494 \mathrm{ft}) \text { and, for the most part, is similar to } \\
\text { above intervals but tends to be slightly } \\
\text { coarser and contains conspicuous white chalky } \\
\text { grains. The siltstone is also similar to the above } \\
\text { intervals. See Table } 23 \text { for detailed description } \\
\text { of Core } \# 3,751.0-757.7 \mathrm{~m}(2464-2486 \mathrm{ft}) \text {. }\end{array}$ & $\begin{array}{l}\text { Eleana Formation, } \\
\text { Unit J (?) }\end{array}$ \\
\hline $\begin{array}{l}760.2-800.1 \mathrm{~m} \\
(2494-2625 \mathrm{ft})\end{array}$ & $\begin{array}{l}\text { Shale with interbedded chert sandstone and } \\
\text { siltstone. Lithologies similar to } \\
\text { above intervals. Minor very-light-gray } \\
\text { chert fragments in the } 777.2 \mathrm{~m}(2550 \mathrm{ft}) \\
\text { sample. The Formation Micro-scanner log } \\
\text { indicates that bedding within the interval } \\
765.0-789.4 \mathrm{~m}(2510-2590 \mathrm{ft}) \text { has a mean } \\
\text { strike of } \mathrm{N} 22^{\circ} \mathrm{E} \text { and a mean dip of } 27^{\circ} \mathrm{NW} \text {. }\end{array}$ & $\begin{array}{l}\text { Eleana Formation, } \\
\text { Unit J (?) }\end{array}$ \\
\hline $\begin{array}{l}800.1-827.8 \mathrm{~m} \\
(2625-2716 \mathrm{ft})\end{array}$ & $\begin{array}{l}\text { Chert sandstone and siltstone. Lithologies } \\
\text { similar to above intervals. Formation } \\
\text { Micro-scanner log indicates that the base of } \\
\text { the quartzite bed at } 816.9 \mathrm{~m}(2680 \mathrm{ft}) \text { strikes } \\
\mathrm{N} 15^{\circ} \mathrm{E} \text { and dips } 28^{\circ} \mathrm{NW} \text {. }\end{array}$ & $\begin{array}{l}\text { Eleana Formation, } \\
\text { Unit J }\end{array}$ \\
\hline $\begin{array}{l}827.8-856.8 \mathrm{~m} \\
(2716-2811 \mathrm{ft})\end{array}$ & $\begin{array}{l}\text { Shale with minor siltstone and } \\
\text { chert sandstone. Lithologies similar to } \\
\text { above intervals. Increase in light-gray chert } \\
\text { fragments in the } 835.2 \mathrm{~m} \text { ( } 2740 \mathrm{ft}) \text { sample. } \\
\text { Faulted lower contact. Formation Micro- } \\
\text { scanner log indicates that this fault strikes } \\
\mathrm{N} 30^{\circ} \mathrm{E} \text { and dips } 78^{\circ} \mathrm{NW} \text {. }\end{array}$ & $\begin{array}{l}\text { Eleana Formation, } \\
\text { Unit J }\end{array}$ \\
\hline $\begin{array}{l}856.8-874.2 \mathrm{~m} \\
(2811-2868 \mathrm{ft})\end{array}$ & $\begin{array}{l}\text { Calcareous siltstone: Grayish-black; moderately } \\
\text { to well indurated; argillaceous; } \\
\text { pyritic; calcareous; common coarsely } \\
\text { crystalline, white calcite as fracture fillings } \\
\text { and free fragments. Rare light-gray chert } \\
\text { fragments. Spectralog indicates interval is } \\
\text { possibly fractured throughout. Faulted upper } \\
\text { and lower contacts. }\end{array}$ & $\begin{array}{l}\text { Eleana Formation }{ }^{1} \text {, } \\
\text { Unit J (?) }\end{array}$ \\
\hline $\begin{array}{l}874.2-932.7 \mathrm{~m} \\
(2868-3060 \mathrm{ft})\end{array}$ & $\begin{array}{l}\text { Dolomite: Grades from light-gray at } \\
\text { top of interval to grayish-black at } \\
\text { base; well indurated; finely to coarsely } \\
\text { crystalline, lighter color tends to be coarse } \\
\text { crystalline; scarce pyrite; no visible porosity; } \\
\text { moderate fetid odor when broken. Thin, } \\
\text { irregular. "tight" dark-gray fracture traces. }\end{array}$ & $\begin{array}{l}\text { Lower Simonson } \\
\text { Dolomite }^{1}\end{array}$ \\
\hline
\end{tabular}


Table 21. Lithologic Log for ER-12-1. (Logged by S. L. Drellack and L. B. Prothro, RSN, 11/91) (continued).

\begin{tabular}{|c|c|c|}
\hline Depth & Lithologic Description & Stratigraphic Unit \\
\hline $\begin{array}{l}874.2-932.7 \mathrm{~m} \\
(2868-3060 \mathrm{ft}) \text { cont. }\end{array}$ & $\begin{array}{l}\text { Macrofossil fragments noted in Core \#4 } \\
\text { are not readily recognizable in cuttings. } \\
\text { Possible fault or fracture zone at } 901.0-910.1 \mathrm{~m} \\
(2956-2986 \mathrm{ft}) \text {. See Table } 24 \text { for description of } \\
\text { Core \#4 from } 895.2-903.0 \mathrm{~m}(2937.0-2962.5 \mathrm{ft}) \text {. } \\
\text { Faulted upper and lower contacts. }\end{array}$ & \\
\hline $\begin{array}{l}932.7-949.4 \mathrm{~m} \\
(3060-3115 \mathrm{ft})\end{array}$ & $\begin{array}{l}\text { Dolomite: Medium-gray at top of } \\
\text { interval to grayish-black at base, } \\
\text { medium crystalline. Possible repeat of } \\
\text { interval } 915.9-932.7 \mathrm{~m}(3005-3060 \mathrm{ft}) \text {. } \\
\text { Faulted upper and lower contacts. }\end{array}$ & $\begin{array}{l}\text { Lower Simonson }{ }^{1} \\
\text { Dolomite }\end{array}$ \\
\hline $\begin{array}{l}949.4-975.4 \mathrm{~m} \\
(3115-3200 \mathrm{ft})\end{array}$ & $\begin{array}{l}\text { Dolomite: Generally light-gray but } \\
\text { varies from very light-gray to medium- } \\
\text { gray; well indurated; medium crystalline, } \\
\text { some coarsely crystalline; very scarce pyrite; } \\
\text { very thin medium-dark-gray fractures and } \\
\text { styolites; no visible porosity; moderate } \\
\text { fetid odor when broken. Samples from } 969.3 \text { - } \\
978.4 \mathrm{~m}(3180-3210 \mathrm{ft}) \text { are contaminated } \\
\text { with dolomite from } 542.5-550.8 \mathrm{~m} \\
(1780-1807 \mathrm{ft}) \text { and quartzite, siltstone, and } \\
\text { shale from } 550.8-856.8 \mathrm{~m}(1807-2811 \mathrm{ft}) \text {. } \\
\text { Probable fault at } 962.6 \mathrm{~m}(3158 \mathrm{ft}) \text {. } \\
\text { Faulted upper contact. }\end{array}$ & $\begin{array}{l}\text { Lower Simonson } \\
\text { Dolomite }^{1}\end{array}$ \\
\hline $\begin{array}{l}975.4-1086.3 \mathrm{~m} \\
(3200-3564 \mathrm{ft})\end{array}$ & $\begin{array}{l}\text { Dolomite: Generally light-gray but varies from } \\
\text { very- light-gray to medium-gray; well indurated; } \\
\text { medium crystalline, some coarsely crystalline; very } \\
\text { scarce pyrite; very thin medium- dark-gray fractures } \\
\text { and styolites; no visible porosity; moderate fetid odor } \\
\text { when broken. Transitional contact with overlaying } \\
\text { Simonson Dolomite. Stratigraphic assignment based } \\
\text { on conodont studies. }\end{array}$ & $\begin{array}{l}\text { Upper Sevy } \\
\text { Dolomite }\end{array}$ \\
\hline $\begin{array}{l}1086.3-1093.6 \mathrm{~m} \\
(3564-3588 \mathrm{ft}) \\
\mathrm{TD}\end{array}$ & $\begin{array}{l}\text { Biotite lamprophyre intrusion: Greenish- } \\
\text { black ( } 5 \mathrm{GY} 2 / 1 \text { ); moderately to well } \\
\text { indurated; coarsely crystalline; very abundant } \\
\text { booklets of mica; common chlorite; abundant } \\
\text { quartz; common feldspar; initially moderately } \\
\text { calcareous, decreasing after a few minutes } \\
\text { to very weakly calcareous: magnetic. } \\
\text { XRD data for the } 1091.2 \mathrm{~m}(3580 \mathrm{ft}) \text { sample } \\
\text { is given in Table } 15 \text {. Formation Micro- } \\
\text { scanner indicates that the upper contact } \\
\text { strikes north and dips } 65^{\circ} \mathrm{W} \text {. }\end{array}$ & Intrusive \\
\hline
\end{tabular}

'Stratigraphic assignment based on paleontological investigations by A.G. Harris, USGS-Reston, VA. 
Table 22. Description of Core \#1 (539.2 to $543.8 \mathrm{~m})$.

\begin{tabular}{lll}
\hline Depth & Lithologic Description & Stratigraphic Unit \\
\hline $539.2-539.3 \mathrm{~m}$ & $\begin{array}{l}\text { Dolomite: Medium-light-gray to } \\
\text { medium-gray and light-olive-gray mottled; } \\
\text { well indurated; finely crystalline; numerous } \\
\text { "tight" hairline fractures with dark-yellowish- } \\
\text { orange stain; succrosic dolomite fracture fill } \\
\text { on some surfaces. Two small, slightly rounded } \\
\text { core fragments recovered. }\end{array}$ & $\begin{array}{l}\text { Upper Simonson } \\
\text { Dolomite or } \\
\text { Lower Guilmette }\end{array}$ \\
& No core recovery. & \\
$539.3-543.8 \mathrm{~m}$ & & \\
$(1769.2-1784.0 \mathrm{ft})$ & & \\
\hline
\end{tabular}

'Stratigraphic assignment based on paleontological investigations by A.G. Harris, USGS, Reston. VA.

that Unit E of Poole and others (1961) is also characterized by fairly uniformly fine-grained sediments and is several hundred feet thick in the northern Eleana Range to the east of ER-12-1, and as much as $2400 \mathrm{ft}$ thick to the northeast in Quartzite Ridge. In the absence of more definitive lithologic or paleontologic information, the correlation of fine-grained siltstone and sandstone in ER-12-1 with Unit $J$ is considered tentative.

An igneous intrusive dike was encountered at $1086.3 \mathrm{~m}$ (3564 ft). Unfortunately, only $7.3 \mathrm{~m}$ $(24 \mathrm{ft})$ were penetrated before total depth was reached within the unit at $1093.6 \mathrm{~m}(3588 \mathrm{ft})$. The Schlumberger Formation Micro-scanner log shows the upper contact dipping $65^{\circ}$ to the west. This orientation is consistent with structural orientations in the region. An age of $102.3 \pm 0.5 \mathrm{Ma}$ was determined by Ar40 - Ar39 analysis of biotite separated from cuttings samples representing this bottom hole unit (M.A. Lanphere, USGS. Menlo Park, CA). This is consistent with emplacement ages for nearby Gold Meadows and Climax granite stocks. Table 26 is a list of the nearest drill holes in the area that penetrated Mesozoic quartz monzonite. Additional discussion regarding this intrusive is given in the following lithology, stratigraphy and paleontology section of this report.

\section{Structural Data}

The structures encountered at ER-12-1 are related to a complex system of faults that are largely the result of Mesozoic Sevier thrust faulting and more recent Basin and Range normal faulting (see preliminary west-east geologic cross section. Figure 4). Tables 27 and 28 provide data on the faults and fracture zones intersected in the ER-12-1 borehole. These faults are probably part of the same system as the Tongue Wash Fault. The Tongue Wash Fault is a west-dipping thrust fault exposed approximately $640.1 \mathrm{~m}$ (2100 ft) southeast of ER-12-1. The fault has placed the Devonian Simonson Dolomite over the Mississippian Eleana Formation. Pre-drill estimates indicated that the Tongue

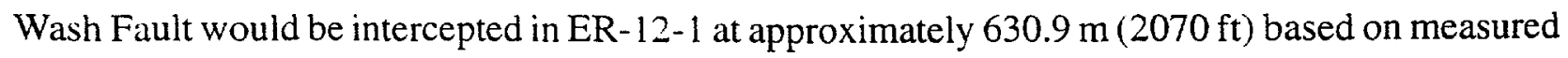


dips of slip surfaces along the fault zone at the surface (Cole, unpublished). Based on the stratigraphy encountered in ER-12-1, the fault intercepted at $312.4 \mathrm{~m}(1025 \mathrm{ft})$ is probably the westward extension of the Tongue Wash Fault. This suggests the Tongue Wash Fault flattens at a shallower depth then previously thought. Since the thrust fault at $550.8 \mathrm{~m}(1807 \mathrm{ft})$ is probably related to the Tongue Wash Fault, it likely has a similar orientation. Significant fracturing appears to be confined

Table 23. Description of Core \#3 (751.0 to $757.7 \mathrm{~m})$.

\begin{tabular}{|c|c|c|}
\hline Depth & Lithologic Description & Stratigraphic Unit \\
\hline $\begin{array}{l}751.0-754.1 \mathrm{~m} \\
(2464-2474 \mathrm{ft})\end{array}$ & $\begin{array}{l}\text { Chert sandstone, black; well indurated } \\
\text { (unconfined compressive strengths up } \\
\text { to } 11,000 \text { psi); dense (bulk density of } \\
2.67 \mathrm{~g} / \mathrm{cc} \text { ); fine-grained, argillaceous (clear, } \\
\text { subrounded, fine-grained quartz in a } \\
\text { black argillaceous matrix); pyritic. } \\
\text { White calcite veins } 0.25 \text { to } 3 \mathrm{~mm} \text { wide } \\
\text { throughout. Distinct calcite veins at: } \\
751.1 \mathrm{~m} \text { ( } 2464.3 \mathrm{ft}), 87^{\circ} \text { dip; } \\
751.7 \mathrm{~m}(2466.3 \mathrm{ft}), 55^{\circ} \text { dip; } \\
751.8 \mathrm{~m}(2466.6 \mathrm{ft}), 73^{\circ} \text { dip; } \\
751.9 \mathrm{~m}(2467.0 \mathrm{ft}), 65^{\circ} \text { dip; } \\
752.1 \mathrm{~m}(2467.6 \mathrm{ft}), 58^{\circ} \text { dip; two at } \\
752.5 \mathrm{~m}(2468.7 \mathrm{ft}), 45^{\circ} \text { and } 65^{\circ} \text { dips; } \\
753.4 \mathrm{~m}(2471.8 \mathrm{ft}), 60^{\circ} \text { dip; and } \\
753.9 \mathrm{~m}(2473.4 \mathrm{ft}), 25^{\circ} \text { dip. } \\
\text { Core parted along fractures with dis- } \\
\text { continous pyrite and calcite coatings } \\
\text { at: } 751.2 \mathrm{~m}(2464.7 \mathrm{ft}), 78^{\circ} \text { dip; } \\
751.5 \mathrm{~m}(2465.4 \mathrm{ft}), 80^{\circ} \text { dip; } \\
752.3 \mathrm{~m}(2468.3 \mathrm{ft}), 57^{\circ} \text { dip; and } \\
753.2 \mathrm{~m}(2471.0 \mathrm{ft}), 88^{\circ} \text { dip. } \\
\text { The recovered core from this run is } \\
\text { well broken. The average core segment } \\
\text { is about } 0.09 \mathrm{~m}(0.3 \mathrm{ft}) \text { long, } \\
\text { with relatively flat ends dipping } \\
3 \text { to } 5^{\circ} . \text { These ends are smoothed, } \\
\text { indicating that the core segments have } \\
\text { rotated against each other during coring. }\end{array}$ & $\begin{array}{l}\text { Eleana Formation, } \\
\text { Unit J (?) }\end{array}$ \\
\hline
\end{tabular}

$754.1-757.7 \mathrm{~m}$

No core recovery. $(2474-2486 \mathrm{ft})$ 
Table 24. Description of Core \#4 (895.2 - $903.1 \mathrm{~m})$.

\begin{tabular}{|c|c|c|}
\hline Depth & Lithologic Description & Stratigraphic Unit \\
\hline $\begin{array}{l}895.2-895.8 \mathrm{~m} \\
(2937.0-2940.3 \mathrm{ft})\end{array}$ & $\begin{array}{l}\text { Dolomite: Medium-light-gray to light- } \\
\text { gray; finely crystalline; dense; } \\
\text { common dark-gray hairline fractures less than } \\
1 \mathrm{~mm} \text { thick. Fracture at } 895.2 \mathrm{~m}(2937.1 \mathrm{ft}) \\
\text { dips } 65^{\circ} \text { with trace of vuggy porosity. } \\
\text { Fractures at } 895.4 \mathrm{~m}(2937 \mathrm{ft}) \text { dip } 35^{\circ} \\
\text { with } 1-\mathrm{mm} \text {-thick dark-gray argillaceous } \\
\text { fracture fill. Two stylolites at } 895.7 \mathrm{~m} \\
\text { ( } 2938.6 \mathrm{ft}) \text {, one is offset by fractures and } \\
\text { has a dip of } 40^{\circ} \text {. At } 855.7-895.9 \mathrm{~m} \\
\text { ( } 2938.8-2939.2 \mathrm{ft}), \text { stylolites dip } 40^{\circ} \text {; } \\
\text { typically filled with similar dark-gray } \\
\text { argillaceous material as mentioned above in the } \\
\text { hairline fractures. }\end{array}$ & $\begin{array}{l}\text { Lower Simonson } \\
\text { Dolomite }^{1}\end{array}$ \\
\hline $\begin{array}{l}895.8-896.2 \mathrm{~m} \\
(2939.0-2940.3 \mathrm{ft})\end{array}$ & $\begin{array}{l}\text { Dolomite: Medium-light-gray to medium- } \\
\text { gray; fine crystalline. Stylolites at } 896 \mathrm{~m} \\
(2939.6 \mathrm{ft}) \text { with dark-gray argillaceous } \\
\text { material plus discontinuous pyrite; dip is } 50^{\circ} \text {, } \\
\text { and separates two shades of gray dolomite. }\end{array}$ & $\begin{array}{l}\text { Lower Simonson } \\
\text { Dolomite }^{1}\end{array}$ \\
\hline $\begin{array}{l}896.2-897.0 \mathrm{~m} \\
(2940.3-2943.0 \mathrm{ft})\end{array}$ & $\begin{array}{l}\text { Dolomite: Medium-dark-gray; finely } \\
\text { crystalline; lower contact dips } 50^{\circ} \text {. At } 896.2 \mathrm{~m} \\
\text { ( } 2940.4 \mathrm{ft} \text { ), a fracture dips } 70^{\circ} \text {; core broke } \\
\text { along with this fracture. At } 896.5 \mathrm{~m} \text { ( } 2941.4 \mathrm{ft} \text { ), } \\
\text { tight fractures dip about } 80^{\circ} \text {; macro-fossil } \\
\text { fragments including crinoid stems, brachiopod } \\
\text { and/or pelecypod. Conspicuous macro-fossils } \\
\text { at } 896.7 \text { - } 897.0 \mathrm{~m} \text { ( } 2942 \text { - } 2943 \mathrm{ft}) \text {. At } 897.0 \mathrm{~m} \\
\text { (2943 ft) a } 1 \text {-cm-thick light-gray band dips } 20^{\circ} \text {. }\end{array}$ & $\begin{array}{l}\text { Lower Simonson } \\
\text { Dolomite }^{1}\end{array}$ \\
\hline $\begin{array}{l}897.0-898.7 \mathrm{~m} \\
(2943.0-2948.4 \mathrm{ft})\end{array}$ & $\begin{array}{l}\text { Dolomite: Mottled in appearance, very-light } \\
\text { to light-gray and medium-gray to medium- } \\
\text { dark-gray; fossiliferous including brachiopods } \\
\text { and crinoids. Discontinuous stylolites at } 898.2 \mathrm{~m} \\
(2947 \mathrm{ft}) \text {. At } 898.4 \mathrm{~m}(2947.5 \mathrm{ft}) \text {, a fracture with } \\
\text { minor displacement dips } 70^{\circ} \text {. At } 898.5 \mathrm{~m}(2947.8 \mathrm{ft}) \text {, } \\
\text { a stylolite dips } 55^{\circ} \text { with several centimeters } \\
\text { of offset, appears tight. }\end{array}$ & $\begin{array}{l}\text { Lower Simonson } \\
\text { Dolomite } \\
\text {, }\end{array}$ \\
\hline $\begin{array}{l}898.7-899.9 \mathrm{~m} \\
(2948.4-2952.5 \mathrm{ft})\end{array}$ & $\begin{array}{l}\text { Dolomite: Light-gray to medium-light-gray; } \\
\text { fine crystalline; common irregular hairline } \\
\text { fractures filled with dark-gray argillaceous } \\
\text { material; highly fractured area at } 899.3 \mathrm{~m} \\
\text { ( } 2950.4 \mathrm{ft} \text { ) on one side of core. A fracture at } \\
899.3 \mathrm{~m}(2950.5 \mathrm{ft}) \text { dips } 45^{\circ} \text {. Possible bedding dips } \\
45^{\circ} \text { and is fractured. Stylolite at } 899.6 \mathrm{~m}(2951.3 \mathrm{ft}) \text {. }\end{array}$ & $\begin{array}{l}\text { Lower Simonson } \\
\text { Dolomite }^{1}\end{array}$ \\
\hline
\end{tabular}


Table 24. Description of Core \#4 (895.2 - $903.1 \mathrm{~m})$ (continued).

\begin{tabular}{|c|c|c|}
\hline Depth & Lithologic Description & Stratigraphic Unit \\
\hline $\begin{array}{l}899.9-900.7 \mathrm{~m} \\
(2952.5-2955.1 \mathrm{ft})\end{array}$ & $\begin{array}{l}\text { Dolomite: Medium-dark-gray to light- } \\
\text { gray; mottled; indistinguishable fossil } \\
\text { fragments including a possible rugose } \\
\text { coral at } 900.2 \mathrm{~m}(2953.3 \mathrm{ft}) \text {. Upper } \\
\text { contact dips } 50^{\circ} \text {. At } 900.4 \mathrm{~m} \\
\text { ( } 2953.9 \mathrm{ft}) \text {, the core is broken along } \\
\text { a fracture that dips } 80^{\circ} \text {, another } \\
\text { fracture healed with } 1-\text { to } 2-\mathrm{mm} \text { thick } \\
\text { recrystallized dolomite dips } 30^{\circ} \text { and } \\
\text { displays 1- to } 4-\mathrm{mm} \text { offset due to two } \\
\text { other perpendicular fractures. } \\
\text { Fracture at } 900.5 \mathrm{~m}(2954.4 \mathrm{ft}) \text { dips } 50^{\circ} \text {. }\end{array}$ & $\begin{array}{l}\text { Lower Simonson } \\
\text { Dolomite }^{1}\end{array}$ \\
\hline $\begin{array}{l}900.7-901.0 \mathrm{~m} \\
(2955.1-2956.0 \mathrm{ft})\end{array}$ & $\begin{array}{l}\text { Dolomite: Dark-gray; finely crystal- } \\
\text { line. At } 900.7 \mathrm{~m}(2954.9 \mathrm{ft}) \text { vuggular } \\
\text { porosity that range from } 2 \mathrm{~mm} \text { to } 1 \mathrm{~cm} \\
\text { in diameter. }\end{array}$ & $\begin{array}{l}\text { Lower Simonson } \\
\text { Dolomite }^{1}\end{array}$ \\
\hline $\begin{array}{l}901.0-902.1 \mathrm{~m} \\
(2956.0-2959.5 \mathrm{ft})\end{array}$ & $\begin{array}{l}\text { Dolomite: Dark-gray, mottled with } \\
\text { very light-gray; increase in brachiopod } \\
\text { and other macro-fossil fragments. } \\
\text { Secondary vuggy porosity at } 901.2 \mathrm{~m} \\
(2956.6 \mathrm{ft}) \text { is about } 1 \mathrm{~cm} \text { long and } \\
0.5 \mathrm{~cm} \text { wide. At } 901.6 \mathrm{~m} \text { ( } 2958 \mathrm{ft}) \text {, } \\
\text { the mottled appearance stops and high- } \\
\text { angle fractures appear. Fracture at } \\
901.9 \mathrm{~m}(2959 \mathrm{ft}) \text { dips } 70^{\circ} \text {. At } 902 \mathrm{~m} \\
(2959.3 \mathrm{ft}) \text {, vuggy porosity along } \\
\text { irregular fracture. }\end{array}$ & $\begin{array}{l}\text { Lower Simonson } \\
\text { Dolomite }^{1}\end{array}$ \\
\hline $\begin{array}{l}902.1-903.0 \mathrm{~m} \\
(2959.5-2962.5 \mathrm{ft})\end{array}$ & $\begin{array}{l}\text { Dolomite: Dark-gray; very finely } \\
\text { crystalline; argillaceous(?); well } \\
\text { fractured. Upper contact is possible } \\
\text { fault dipping } 45^{\circ} \text {. Several fractures } \\
\text { are filled with light-gray recrystal- } \\
\text { lized dolomite less than } 1 \text { to } 3 \mathrm{~mm} \text { in } \\
\text { width. Fractures are generally healed } \\
\text { with no visible porosity. Multiple } \\
\text { fracture sets causing } 1 \text {-mm to } 1 \text {-cm } \\
\text { offset; fracturing increases downward. }\end{array}$ & $\begin{array}{l}\text { Lower Simonson } \\
\text { Dolomite }\end{array}$ \\
\hline $\begin{array}{l}903.0-903.1 \mathrm{~m} \\
(2962.5-2963.0 \mathrm{ft})\end{array}$ & $\begin{array}{l}\text { No core recovery. (Fractured } \\
\text { interval, possible fault) }\end{array}$ & \\
\hline
\end{tabular}

\footnotetext{
${ }^{1}$ Stratigraphic assignment based on paleontological investigations by A.G. Harris, USGS-Reston, VA.
} 
Table 25. Selected Holes that Penetrate the Pre-Tertiary Rocks Surrounding ER-12-1.

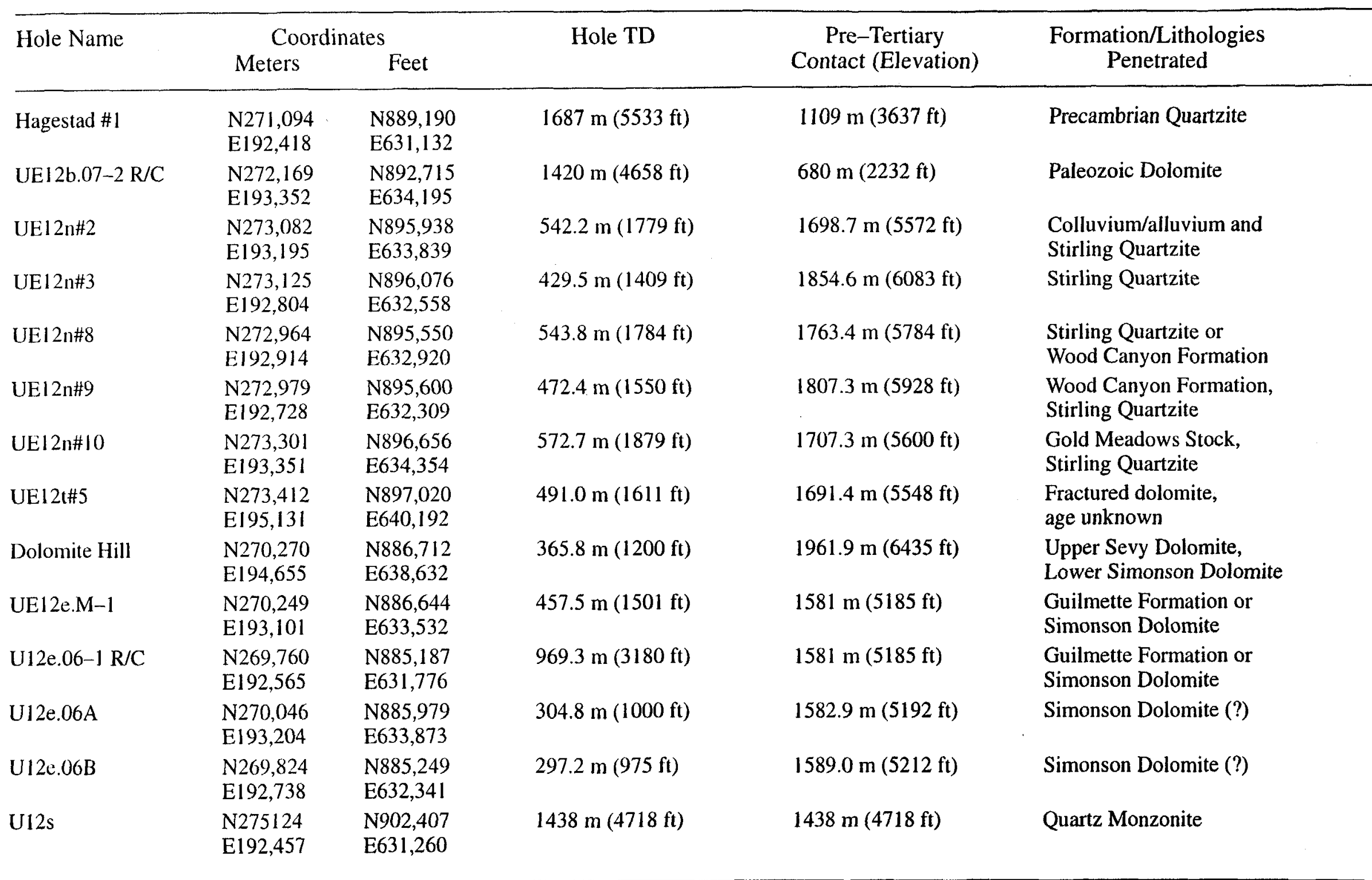

Information from: Schoff and Winograd, 1973; Maldonado et al., 1979; and Emerick and Dickey, 1961; Cole, unpublished data. 
Table 26. Rainier Mesa Drill Holes Penetrating Quartz Monzonite (Gold Meadows Stock).

\begin{tabular}{lccccc}
\hline Drill Hole & $\begin{array}{c}\text { Collar } \\
\text { Elevation } \\
\text { MSL }\end{array}$ & $\begin{array}{c}\text { Depth } \\
\text { BGL }\end{array}$ & $\begin{array}{c}\text { Elevation } \\
\text { MSL }\end{array}$ & Northing & Easting \\
\hline Rainier Mesa & $\begin{array}{l}2245.5 \mathrm{~m} \\
(7367 \mathrm{ft})\end{array}$ & $\begin{array}{l}1108.3 \mathrm{~m} \\
(3636 \mathrm{ft})\end{array}$ & $\begin{array}{c}1137.2 \mathrm{~m} \\
(3731 \mathrm{ft})\end{array}$ & $\begin{array}{l}271,911.2 \mathrm{~m} \\
(892,097 \mathrm{ft})\end{array}$ & $\begin{array}{l}191,842.3 \mathrm{~m} \\
(629,404 \mathrm{ft})\end{array}$ \\
& & & & & \\
UE12p\#1 & $1974.2 \mathrm{~m}$ & $602.3 \mathrm{~m}$ & $1371.9 \mathrm{~m}$ & $276,280.5 \mathrm{~m}$ & $196,543.3 \mathrm{~m}$ \\
& $(6477 \mathrm{ft})$ & $(1976 \mathrm{ft})$ & $(4501 \mathrm{ft})$ & $(906,432 \mathrm{ft})$ & $(644,827 \mathrm{ft})$ \\
& & & & & \\
U12R & $2291.2 \mathrm{~m}$ & $618.7 \mathrm{~m}$ & $1672.4 \mathrm{~m}$ & $272,918.2 \mathrm{~m}$ & $196,543.3 \mathrm{~m}$ \\
& $(7517 \mathrm{ft})$ & $(2030 \mathrm{ft})$ & $(5487 \mathrm{ft})$ & $(895,401 \mathrm{ft})$ & $(628,500 \mathrm{ft})$ \\
UE12n\#10 & $2250.6 \mathrm{~m}$ & $544.1 \mathrm{~m}$ & $1706.6 \mathrm{~m}$ & $273,300.7 \mathrm{~m}$ & $193,351.1 \mathrm{~m}$ \\
& $(7384 \mathrm{ft})$ & $(1785 \mathrm{ft})$ & $(5599 \mathrm{ft})$ & $(896,656 \mathrm{ft})$ & $(634,354 \mathrm{ft})$ \\
UE12n\#15A & $2246.1 \mathrm{~m}$ & $574.9 \mathrm{~m}$ & $1671.2 \mathrm{~m}$ & $272,719.5 \mathrm{~m}$ & $192,535.5 \mathrm{~m}$ \\
& $(7369 \mathrm{ft})$ & $(1886 \mathrm{ft})$ & $(5483 \mathrm{ft})$ & $(894,794 \mathrm{ft})$ & $(631,678 \mathrm{ft})$ \\
\hline
\end{tabular}

MSL - Mean Sea Level

BGL - Below Ground Level

Information from Maldonado et al., 1979; and Townsend, 1991 (verbal communication)

mostly to the dolomite units and the intrusive, except near faults where fracturing usually increases independent of lithology. Bedding in the borehole generally dips in a westerly direction at an angle of $25-30^{\circ}$. This orientation is consistent with that measured at the surface in the vicinity of ER-12-1.

Other structural solutions, for the observations made at ER-12-1, may be acceptable. For example, the Tongue Wash Fault can be projected from the surface to the fault zone at 521 to 552 $\mathrm{m}(1710-1810 \mathrm{ft})$ at a constant dip of about $45^{\circ}$. This interpretation would imply that it is younger than the faults that shuffle the stratigraphy in the upper part of the hole, which is consistent with the surface observation that the most recent movement on the Tongue Wash Fault was dominantly strike-slip (left-lateral, with a component of reverse slip).

\section{STRATIGRAPHY, STRUCTURE, AND THERMAL HISTORY OF ROCKS PENETRATED BY WELL ER-12-1 BASED UPON MICROSCOPIC OBSERVATIONS}

This section describes the results of several related, detailed studies of cuttings from well ER-12-1 and includes certain observations made during field investigations in and around the well site. The cuttings samples that form the basis of these studies were obtained in one-gallon metal cans at each $15.2 \mathrm{~m}(50-\mathrm{ft})$ advance of the drill bit, and were collected in addition to the two sets of cuttings 


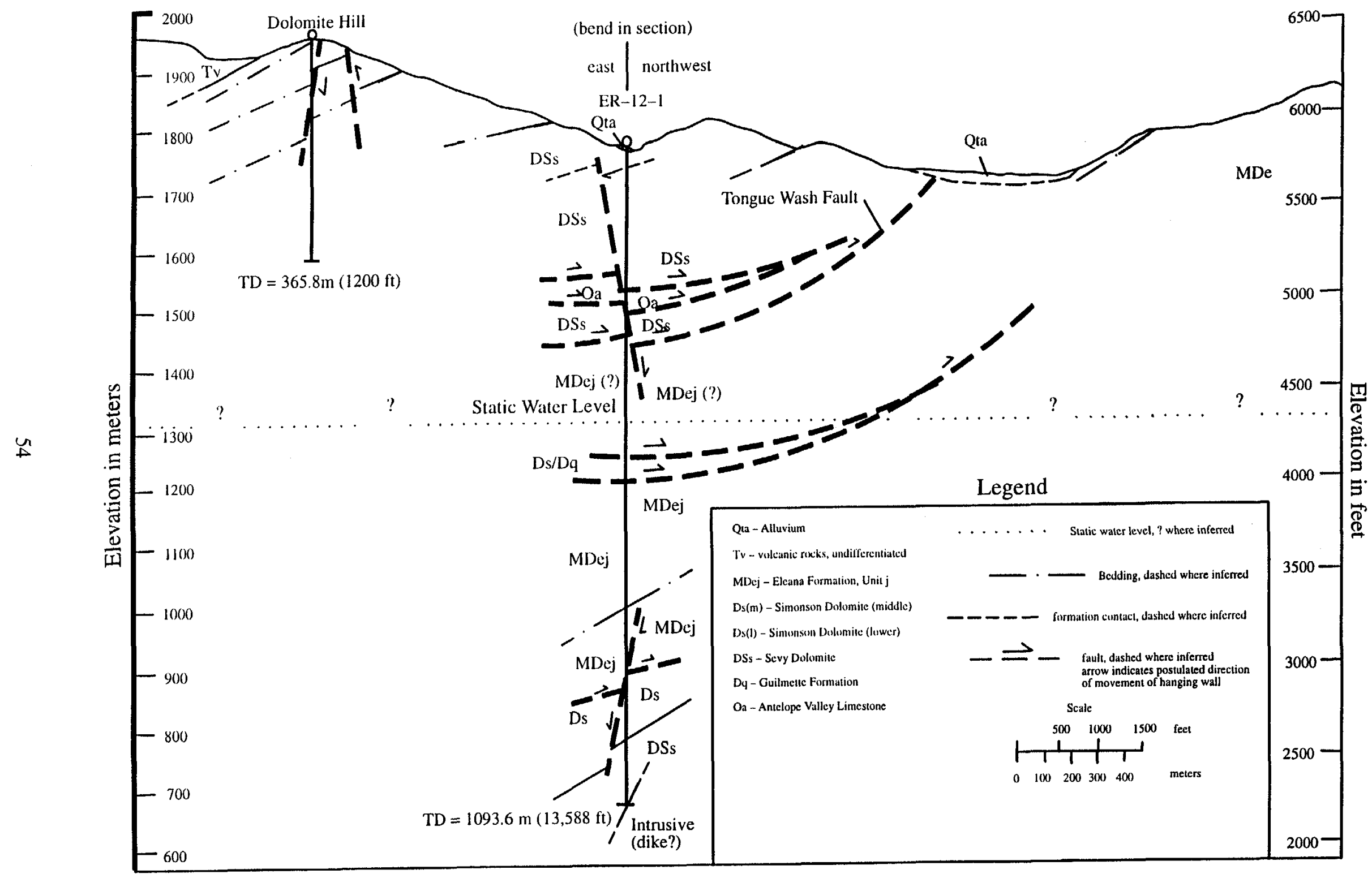

Figure 4. Preliminary west-east geologic cross section for ER-12-1. (Includes recent stratigraphic assignments based on paleontological analysis by A.G. Harris, USGS-Reston, VA.) 
Table 27. Probable Faults Intersected in the ER-12-1 Borehole.

\begin{tabular}{|c|c|c|c|c|c|c|c|c|}
\hline \multicolumn{2}{|c|}{ Depth } & \multicolumn{2}{|c|}{ Formations } & \multicolumn{2}{|c|}{ Lithologies } & \multirow[t]{2}{*}{ Strike } & \multirow[t]{2}{*}{ Dip } & \multirow[t]{2}{*}{ Indicators* } \\
\hline Meters & Feet & Hanging Wall & Footwall & Hanging Wall & Footwall & & & \\
\hline 237.7 & 780 & $\begin{array}{l}\text { Lower Sevy } \\
\text { Dolomite }\end{array}$ & $\begin{array}{l}\text { Antelope Valley } \\
\text { Limestone }\end{array}$ & Dolomite & Dolomite & NA & NA & $a, b$ \\
\hline 259.1 & 850 & $\begin{array}{l}\text { Antelope Valley } \\
\text { Limestone }\end{array}$ & $\begin{array}{l}\text { Antelope Valley } \\
\text { Limestone }\end{array}$ & Dolomite & Dolomite & NA & NA & $a, b, c$ \\
\hline 274.3 & 900 & $\begin{array}{l}\text { Antelope Valley } \\
\text { Limestone }\end{array}$ & $\begin{array}{l}\text { Lower Sevy } \\
\text { Dolomite }\end{array}$ & Dolomite & Dolomite & NA & NA & $\mathrm{a}, \mathrm{b}$ \\
\hline 312.4 & 1025 & $\begin{array}{l}\text { Sevy } \\
\text { Dolomite }\end{array}$ & $\begin{array}{l}\text { Eleana, } \\
\text { Unit } J\end{array}$ & Dolomite & $\begin{array}{l}\text { Calcareous } \\
\text { Siltstone }\end{array}$ & NA & NA & a \\
\hline 521.2 & 1710 & $\begin{array}{l}\text { Eleana, } \\
\text { Unit } \mathbf{J}(?)\end{array}$ & $\begin{array}{l}\text { Upper Simonson } \\
\text { Dolomite-Lower } \\
\text { Guilmette Form. }\end{array}$ & Siltstone & Dolomite & NA & NA & $a, b, c$ \\
\hline 533.4 & 1750 & $\begin{array}{l}\text { Simonson } \\
\text { Dolomite }\end{array}$ & $\begin{array}{l}\text { Simonson } \\
\text { Dolomite }\end{array}$ & Dolomite & Dolomite & NA & NA & b, c \\
\hline 543.1 & 1782 & $\begin{array}{l}\text { Simonson } \\
\text { Dolomite }\end{array}$ & $\begin{array}{l}\text { Simonson } \\
\text { Dolomite }\end{array}$ & Dolomite & Dolomite & NA & NA & $b, c$ \\
\hline 550.8 & 1807 & $\begin{array}{l}\text { Simonson } \\
\text { Dolomite }\end{array}$ & $\begin{array}{l}\text { Eleana, } \\
\text { Unit J }\end{array}$ & Dolomite & Chert Sandstone & NA & NA & $a, b, c$ \\
\hline 856.8 & 2811 & $\begin{array}{l}\text { Eleana, } \\
\text { Unit J }\end{array}$ & $\begin{array}{l}\text { Eleana, } \\
\text { Unit } J(?)\end{array}$ & Shale & $\begin{array}{l}\text { Calcareous } \\
\text { Siltstone }\end{array}$ & $\mathrm{N} 30^{\circ} \mathrm{E}$ & $78^{\circ} \mathrm{NW}$ & $a, c$ \\
\hline 874.2 & 2868 & $\begin{array}{l}\text { Eleana, } \\
\text { Unit J(?) }\end{array}$ & $\begin{array}{l}\text { Lower Simonson } \\
\text { Dolomite }\end{array}$ & Siltstone & Dolomite & NA & NA & $\mathrm{a}, \mathrm{c}$ \\
\hline 932.7 & 3060 & $\begin{array}{l}\text { Lower Simonson } \\
\text { Dolomite }\end{array}$ & $\begin{array}{l}\text { Lower Simonson } \\
\text { Dolomite }\end{array}$ & Dolomite & Dolomite & NA & NA & $a, c, d$ \\
\hline 949.4 & 3115 & $\begin{array}{l}\text { Lower Simonson } \\
\text { Dolomite }\end{array}$ & $\begin{array}{l}\text { Lower Simonson } \\
\text { Dolomite }\end{array}$ & Dolomite & Dolomite & NA & NA & b, c \\
\hline 926.6 & 3158 & $\begin{array}{l}\text { Lower Simonson } \\
\text { Dolomite }\end{array}$ & $\begin{array}{l}\text { Lower Simonson } \\
\text { Dolomite }\end{array}$ & Dolomite & Dolomite & NA & NA & $c, d$ \\
\hline $\begin{array}{l}a-\text { strati } \\
b-\text { lithol }\end{array}$ & & $\begin{array}{l}\mathrm{c} \text { - geophysical lo } \\
\mathrm{d} \text { - drilling data }\end{array}$ & & $\mathrm{NA}-\mathrm{Not}$ & & & & \\
\hline
\end{tabular}


Table 28. Possible Faults and/or Fractured Intervals Intersected in the ER-12-1 Borehole.

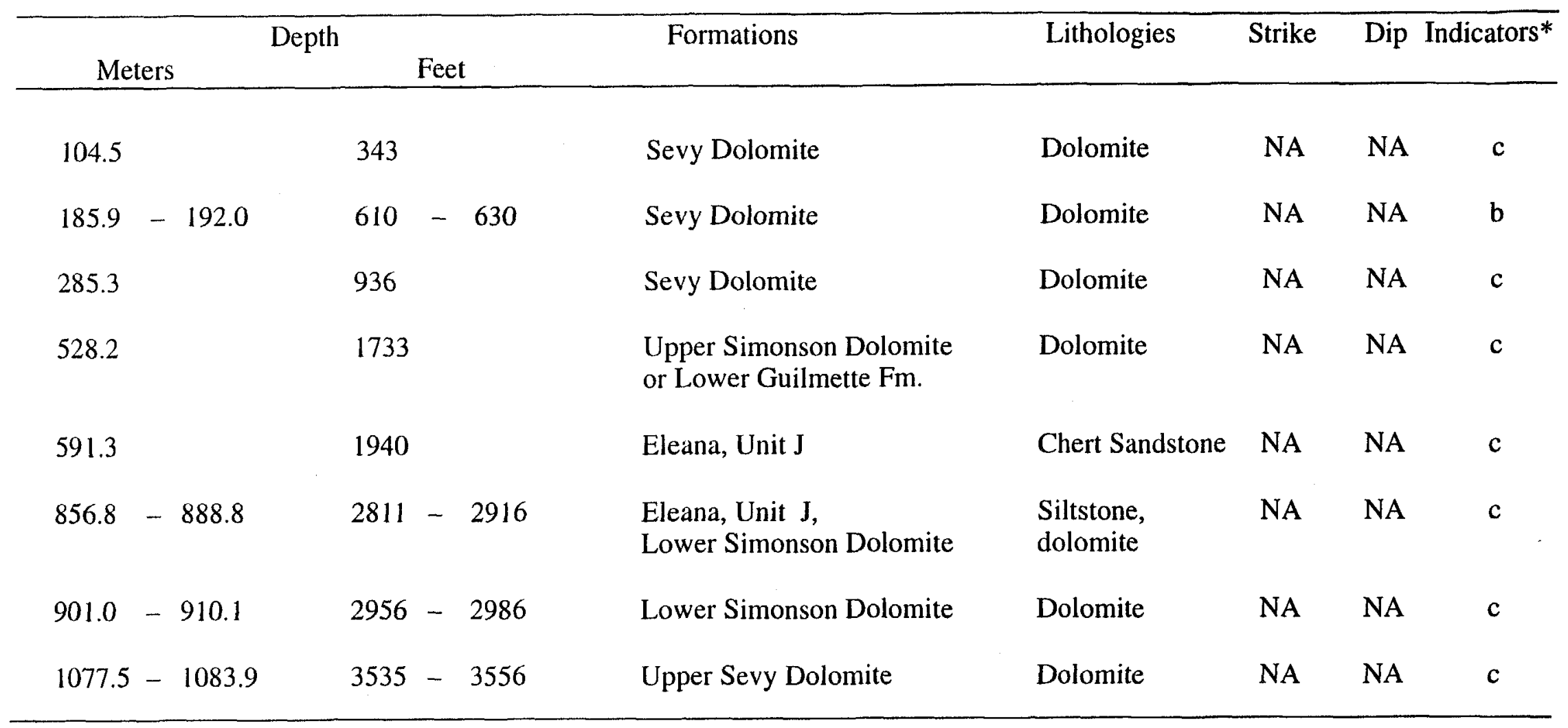

* a - stratigraphy

NA - Not available

b - lithology

c - geophysical logs

d - drilling data 
(archived and washed samples) obtained for each $3 \mathrm{~m}$ (10 ft) advance (Drellack et al., 1991). These cuttings were washed over a 10-mesh sieve to remove drilling mud and excess fines, repackaged, and forwarded to the U.S. Geological Survey offices in Denver, Colorado.

At the USGS in Denver, cuttings (about $20 \mathrm{gm}$ ) were selected from each of the sample containers, bagged, and sent to a private contractor to prepare standard glass-covered petrographic microscope thin sections. All of the remaining sample material was shipped to the Reston, Virginia, laboratories of the USGS for evaluation and processing for non-calcareous microfossils. Each container of carbonate rock cuttings (as well as several containers of calcareous siltstone) was digested in warm acetic acid, and conodonts were hand-picked from the insoluble residue for more detailed identification and examination. The results of those analyses are summarized in Table 29 and are discussed in a following section.

\section{LITHOLOGY, STRATIGRAPHY, AND PALEONTOLOGY}

The rocks penetrated by the ER-12-1 drillhole are a complex assemblage of Ordovician, Silurian, Devonian, and Mississippian sedimentary rocks that are bounded by numerous faults that show substantial stratigraphic offset. The final $7.3 \mathrm{~m}(24 \mathrm{ft})$ of this hole penetrated an unusual intrusive rock of Cretaceous age, as described below. All of these units have been well described in terms of their macroscopic characteristics in the lithologic log prepared by Raytheon Services Nevada (Drellack et al., 1991; Table 21), and so this section of the report only covers additional observations acquired from the prepared thin sections.

The Sevy Dolomite in the upper $237.7 \mathrm{~m}$ ( $780 \mathrm{ft}$ ) of the hole consists of generally mediumto coarse-grained recrystallized dolostone with local beds or zones that contain rounded detrital quartz and secondary pyrite. The term "dolostone" is used here due to the pervasive recrystallization textures, the common occurrence of rhombic crystals of dolomite, and prior studies of the Sevy tidal-flat depositional environment (Osmond, 1954). Original shell fragments are only indicated by sparse dolomitized pseudomorphs. Primary porosity (following post-depositional replacement by dolomite) was probably low, but numerous veins of coarse calcite, chalcedony, and quartz traverse these fragments and attest to prior secondary permeability and groundwater activity.

Cuttings collected at $237.7 \mathrm{~m} \mathrm{(780} \mathrm{ft)} \mathrm{and} 240.8 \mathrm{~m}(790 \mathrm{ft})$ contain numerous fragments of ocher and red-brown dolomite breccia that indicate the presence of a significant fault. Some fragments show slickenside lineations on their broken surfaces. Microscopic examination shows that the breccia formed by brittle shattering and comminution of dolostone with relatively little milling and shearing; recrystallization of the carbonate minerals is also minimal. Veins and replacement patches of quartz are present, and the breccia matrix is irregularly stained with secondary iron-oxide minerals (probably derived from alteration of pyrite).

Between about $240.8 \mathrm{~m}$ and $271.3 \mathrm{~m}$ (790 ft and $890 \mathrm{ft}$ ), cuttings recovered from ER-12-1 consist of heterogeneous, varicolored dolostone and dolomitic limestone and brecciated varieties of both; fragments of quartz-calcite-chalcedony veins are also common. These lithologic 
Table 29. Stratigraphic Log for Groundwater Characterization Project Well ER-12-1 based on Paleontologic and Petrographic Examinations of Cuttings Samples Collected at $15.4 \mathrm{~m}(50-\mathrm{ft})$ Intervals ${ }^{1}$.

\begin{tabular}{|c|c|c|c|}
\hline Sample Depth & Formation & Age & $\begin{array}{l}\text { Conodon } \\
\mathrm{CAI}^{(2)}\end{array}$ \\
\hline $\begin{array}{l}15-137 \mathrm{~m} \\
(50-450 \mathrm{ft})\end{array}$ & $\begin{array}{l}\text { Lower Sevy } \\
\text { Dolomite }\end{array}$ & $\begin{array}{l}\text { Early } \\
\text { Devonian }\end{array}$ & 4 to 5.5 \\
\hline $\begin{array}{l}152-244 \mathrm{~m} \\
(500-800 \mathrm{ft})\end{array}$ & $\begin{array}{l}\text { lower Sevy } \\
\text { Dolomite }\end{array}$ & Late Silurian & 5 to 5.5 \\
\hline
\end{tabular}

\section{Medium-gray to gray-brown, fine- to medium-grained dolostone with variable quartz content in silty layers, sparse fossil forms largely replaced by recrystallization; local stylolites; primary voids locally filled with chalcedony and coarse calcite.}

Equivalent to Unit $F$ and the uppermost part of Unit $E$ of Dolomite of the Spotted Range; late Lockhovian at 15 and $91 \mathrm{~m}$ (50 and $300 \mathrm{ft})$; early Lockhovian at 106-137 m (350-450 ft.) Relatively shallow water, partly restricted marine environment.

Mostly medium-brown-gray, medium-grained, variably sandy dolostone (some fragments contain as much as $15 \%$ rounded quartz silt in fine layers); brittle breccia fabrics increasingly common downward in this interval, and dolostone breccia is traversed by coarse-grained calcite-quartz veins (veins generally post-date brecciation, but locally show slight strain).

Probably equivalent to Unit E of Dolomite of the Spotted Range; relatively shallow water, normal marine environment; sample at $244 \mathrm{~m}$ $(800 \mathrm{ft}$ ) only produced one conodont element of Silurian aspect (may be downhole contaminant). 
Table 29. Stratigraphic Log for Groundwater Characterization Project Well ER-12-1 based on Paleontologic and Petrographic Examinations of Cuttings Samples Collected at $15.4 \mathrm{~m}(50-\mathrm{ft})$ Intervals $^{1}$ (continued).

\begin{tabular}{lllll}
\hline Sample Depth & Formation & Age & $\begin{array}{l}\text { Conodont } \\
\text { CAI }\end{array}$ & \multicolumn{1}{c}{ Lithologic, Stratigraphic, Structural Notes } \\
$\begin{array}{l}259.1-274.3 \mathrm{~m} \\
(850-900 \mathrm{ft})\end{array}$ & $\begin{array}{l}\text { Antelope Valley } \\
\text { Limestone } \\
\text { (Aysees Peak } \\
\text { member) }\end{array}$ & $\begin{array}{l}\text { Middle } \\
\text { Ordovician }\end{array}$ & 5 to 5.5 & $\begin{array}{l}\text { Brown, ocher and red-brown, strongly brecciated dolomitic limestone(?); } \\
\text { some fragments show slickensided surfaces; abundant } \\
\text { quartz-chalcedony-calcite veins traverse breccia fragments } \\
\text { Late early Whiterockian age; conodonts indicate cool water, open-marine } \\
\text { depositional environment; sample at 274 m (900 ft) contains some middle } \\
\text { Devonian to early Mississippian(?) forms interpreted to have come from } \\
\text { tectonically mixed Simonson(?) Dolomite within this fault zone }\end{array}$ \\
& & & &
\end{tabular}

FAULT CONTACT noted in $30 \mathrm{~m}$ (10-ft) interval cuttings between 271 and $274 \mathrm{~m}(890 \mathrm{ft}$ and $900 \mathrm{ft}$ )

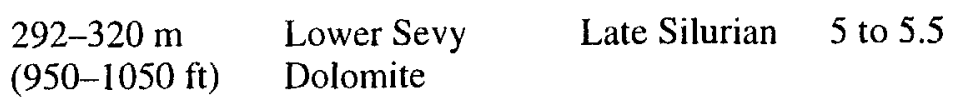

Dark gray to gray brown, medium- to fine-grained dolostone; calcite veins common in upper part but rocks generally not much deformed; minor shell forms preserved.
\end{abstract}

Probably equivalent to Unit $E$ of Dolomite of the Spotted Range; warm, relatively shallow water, normal marine depositional environment. Sample at $320 \mathrm{~m}(1050 \mathrm{ft})$ contains a few indeterminate conodont fragments but abundant siliceous siltstone in the insoluble residue (thus this sample was collected in part below the next major fault contact). 
Table 29. Stratigraphic Log for Groundwater Characterization Project Well ER-12-1 based on Paleontologic and Petrographic Examinations of Cuttings Samples Collected at $15.4 \mathrm{~m}$ (50-ft) Intervals ${ }^{1}$ (continued).

\begin{tabular}{llll}
\hline Sample Depth & Formation & Age & $\begin{array}{l}\text { Conodont } \\
\mathrm{CAI}^{(2)}\end{array}$ \\
\hline $320-518 \mathrm{~m}$ & Upper Eleana & Late & No Data \\
$(1050-1700 \mathrm{ft})$ & Formation & Mississippian &
\end{tabular}

Lithologic, Stratigraphic, Structural Notes

\author{
Dark gray to black, finely laminated carbonaceous, pyritic, fine-grained \\ siltstone with common irregular patches of calcite cement in matrix; local \\ rhombic grains of secondary brown carbonate mineral (ankerite-siderite \\ solid-solution?). Siltstone shows compaction foliation, disruptive silty \\ calcite veins that probably formed during dewatering and lithification, and \\ some conversion of matrix clay to disordered white mica (illite?).
}

Local deformation fabrics include minor crinkling of the compaction foliation and bedding laminations, fine-grained calcite veins parallel to crinkle planes that show synkinematic fibrous crystal growth, small-scale folds with axial-plane shears, and cross-cutting veins of coarsely crystalline calcite and quartz. The shear fabrics and transposition of bedding seem more common between 503 and $518 \mathrm{~m}(1650$ and $1700 \mathrm{ft})$, and may indicate proximity to a compressional (thrust) fault.

Probably equivalent (in part) to Unit $J$ of Eleana Formation. All samples were barren of conodonts with the exception of the collection at $488 \mathrm{~m}$ $(1600 \mathrm{ft})$ that produced one identifiable Silurian-earliest Devonian element. Thin section from $488 \mathrm{~m}(1600 \mathrm{ft})$ shows several fragments of strongly brecciated, veined dolostone in addition to the more common Eleana calcareous siltstone. Possibility of downhole contamination cannot be ruled out; otherwise, a thin tectonic sliver of dolostone is suggested.

Complex FAULT ZONE noted in $3 \mathrm{~m}(10-\mathrm{ft})$ interval cuttings between 521 and $558 \mathrm{~m}$ (1710 and $1830 \mathrm{ft})$

\author{
533-549 m Upper Simonson Late Middle 5.5 to $6.5 \quad$ Mixed cuttings of black, pyritic siliceous siltstone (probably from \\ $\begin{array}{lll}(1750-1800 \mathrm{ft}) & \begin{array}{l}\text { Dolomite or } \\ \text { lower Guilmette }\end{array} & \text { to early Late } \\ & \text { Devonian }\end{array}$ \\ up-hole) with medium gray-brown, coarse-grained dolostone that shows \\ Formation \\ conspicuous breccia fabrics and orange-pink iron-oxide stain; fragments \\ of calcite-quartz vein material common.
}

Abundant diagnostic conodonts recovered from $549 \mathrm{~m}(1800 \mathrm{ft})$ sample indicate Givetian-Frasnian age; normal marine depositional environment.

FAULT ZONE noted in $30 \mathrm{~m}(10-\mathrm{ft})$ interval cuttings at about 558 or $561 \mathrm{~m}(1830 \mathrm{ft}$ or $1840 \mathrm{ft})$

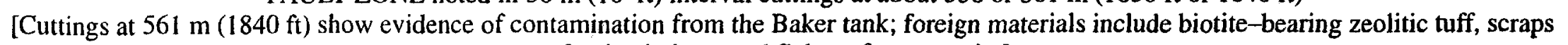
of polyethylene, and flakes of epoxy paint] 
Table 29. Stratigraphic Log for Groundwater Characterization Project Well ER-12-1 based on Paleontologic and Petrographic Examinations of Cuttings Samples Collected at $15.4 \mathrm{~m}$ (50-ft) Intervals ${ }^{1}$ (continued).

\begin{tabular}{llll}
\hline Sample Depth & Formation & Age & $\begin{array}{l}\text { Conodont } \\
\text { CAI }^{(2)}\end{array}$ \\
\hline $564-854 \mathrm{~m}$ & Upper Eleana & Late & No Data \\
$(1850-2800 \mathrm{ft})$ & Formation & Mississippian &
\end{tabular}

Black to dark-gray to variegated gray, fine-grained, siliceous siltstone with irregular carbonate cement and common fresh pyrite; clasts are mostly subrounded and consist of quartz and less common chert and quartz-grain aggregates, and sparse mafic volcanic rock fragments and plagioclase; bedding is inconspicuous and unit is quite uniform over this interval.

Cuttings only show compaction foliation and locally common quartz veins and quartz-calcite veins; no fabrics indicative of significant penetrative deformation.

Probably equivalent (in part) to Unit J of Eleana Formation. Differs notably from Eleana Unit $J$ at $326-518.3 \mathrm{~m}(1070-1700 \mathrm{ft})$ as this lower section is uniformly coarser grained, thicker bedded, more heterolithic, and apparently deposited in much deeper water.

$869-970 \mathrm{~m}$ $(2850-3180 \mathrm{ft})$ Dolomite

(4)
Late Middle to early Late Devonian
5 to 5.5 with some 6.5 to 7

Light-gray to white medium-grained dolostone with common pyrite; gradation to darker gray colors with depth; distinct zone of dark gray, quartz-bearing dolostone at about $927-951 \mathrm{~m}(3040-3120 \mathrm{ft})$.

No conspicuous deformation fabrics, but some cuttings locally show breccia textures; cannot confirm that breccia cuttings belong to this interval due to obvious downhole contamination with Eleana material and with foreign Tertiary volcanic rock.

Latest Emsian to earliest Eifelian age; relatively shallow water, normal marine depositional environment. Variable CAI values and pitted surfaces on conodonts indicate hydrothermal alteration. 
Table 29. Stratigraphic Log for Groundwater Characterization Project Well ER-12-1 based on Paleontologic and Petrographic Examinations of Cuttings Samples Collected at $15.4 \mathrm{~m}(50-\mathrm{ft})$ Intervals $^{1}$ (continued).

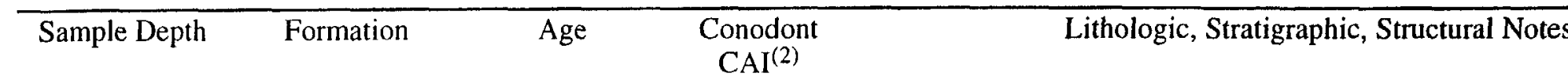

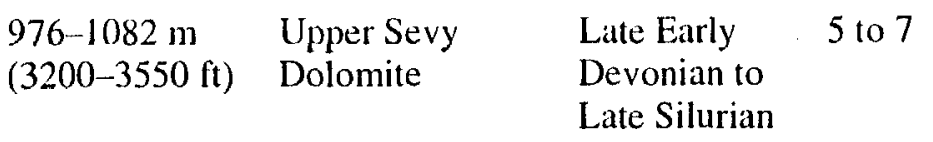

Uniform medium-gray and brownish-gray, coarse-grained dolostone with some pyrite; few veins, fractures, or breccia textures.

Conodonts indicate Emsian and older ages (late Silurian), with no indication of a break or repetition in the sequence; normal marine depositional environment. Variable CAI values and pitted surfaces on conodonts indicate hydrothermal alteration.

Apparent INTRUSIVE CONTACT noted in $3 \mathrm{~m}$ (10-ft) interval cuttings at $1086 \mathrm{~m}$ (3564 ft)(5)

$\begin{array}{llll}1086-1094 \mathrm{~m} & \text { Biotite } & \text { Cretaceous } & \text { No Data } \\ \text { (3564-3588 ft) } & \text { Lamprophyre } & 102.3 \pm 0.5 & \\ \text { (TD) } & & \mathrm{Ma} & \end{array}$

Dark-greenish-gray, altered microporphyritic igneous rock with abundant phenocrysts of plagioclase and biotite; matrix consists of assemblage of secondary chlorite, calcite, white mica, albite, and rutile along with very common accessory magnetite, apatite, and sphene. Overall composition and texture suggest affinity with lamprophyric clan.

Age determined by $\mathrm{Ar}^{40}-\mathrm{Ar}^{39}$ analysis of biotite; see text for details and explanation. 
Table 29. Stratigraphic Log for Groundwater Characterization Project Well ER-12-1 based on Paleontologic and Petrographic Examinations of Cuttings Samples Collected at $15.4 \mathrm{~m}(50-\mathrm{ft})$ Intervals ${ }^{1}$ (continued).

Notes:

(1) Paleontologic investigations supervised by A.G. Harris (USGS Report CRG-92-2, June 4, 1992); petrographic studies by J.C. Cole (USGS, Denver, CO),

R.G. Warren (LANL, Los Alamos, NM);

geochronologic investigation by M.A. Lamphere (USGS, Menlo Park, CA).

(2) $\mathrm{CAI}=$ Color Alteration Index (Epstein et al., 1977) that indicates the maximum temperature to which the conodont sample has been exposed over geologically significant time. CAI values between 4 and 7 indicate exposure temperatures of at least $220^{\circ} \mathrm{C}$ and locally as much as $450^{\circ} \mathrm{C}$.

(3) Identification of faults is based on study of detailed $3 \mathrm{~m}(10-\mathrm{ft})$ interval cuttings by J.C. Cole; faults are indicated by major changes in stratigraphic units, by presence of fragments of fault breccia, by oxidation and alteration, and(or) by the presence of cuttings with slickensided surfaces. Depths may differ slightly from those listed in the RSN lithologic log (Drellack et al., 1991), and Table 21, which was also based in part on geophysical logs.

(4) An additional sample was collected between $960-975 \mathrm{~m}$ (3150 ft and $3200 \mathrm{ft})$.

(5) Lithologic contact picked by RSN (Drellack et al., 1991). 
characteristics alone are not especially distinctive, but the paleontologic analysis clearly indicates these cuttings belong to the middle Ordovician Antelope Valley Limestone (Aysees Peak member; Table 29). The dolomitic composition of this interval, in contrast to the primary limestone typical of this formation, is attributed to recrystallization and replacement of calcite during early stages of brecciation.

Beginning at $274.3 \mathrm{~m}(900 \mathrm{ft})$ and continuing to $312.4 \mathrm{~m}$ (1025 ft), ER-12-1 penetrated fairly uniform, medium- to fine-grained dolostone of the lower Sevy Dolomite. Fragments of fault breccia with common quartz-calcite veins are more typical in the upper part of this interval. Otherwise, the dolostone through this interval is rather unremarkable and only contains sparse recrystallized relics of bivalve shells.

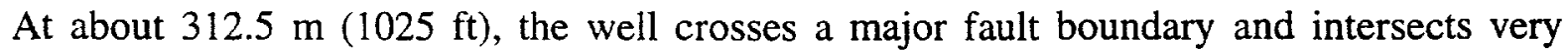
fine-grained, calcareous, carbonaceous, pyritic, laminated siltstones of the Mississippian Eleana Formation. These rocks are characterized by abundant angular to subrounded grains of detrital quartz and lesser chert, by a non-micaceous matrix that consists of compacted clay minerals (montmorillonite?), and by widespread fine-grained, micritic calcite cement. Irregular veinlets of similar micritic calcite crosscut and disrupt the bedding laminations and probably represent diagenetic expulsion of formation water prior to lithification. Some carbonate veinlets of this same apparent period show an internal fibrous structure perpendicular to vein walls that suggests aragonite was growing during vein dilation. Bedding laminations are marked by size variations of detrital grains and by opaque (carbonaceous?) granules and are generally parallel to the compaction foliation defined by crystallographic alignment of matrix clay minerals.

The high degree of carbonate cement and the widespread occurrence of calcite-bearing veins in the upper part of the Eleana Formation are notable. Previously published lithologic log of ER-12-1 (Drellack et al., 1991) noted that, although the lithologic break between Sevy Dolomite above and Eleana Formation below was abrupt at about $312.4 \mathrm{~m}(1025 \mathrm{ft})$, the natural gamma-ray geophysical - log showed transitional characteristics between dolostone and siltstone from about 308 to $323 \mathrm{~m}$ ( $1010 \mathrm{ft}$ to $1060 \mathrm{ft}$ ) (Pawloski and Carison, 1992). Microscopic examination of the cuttings through this interval indicates that the gamma log was most likely dampened by the extensive (non-radioactive) carbonate in the upper Eleana siltstones. The high carbonate content in these rocks accounts for their positive reaction to dilute hydrochloric acid and explains the use of the term "limestone" in the earlier lithologic log (Drellack et al., 1991) for this interval, but there is no doubt that these were deposited as clastic sediments.

The primary depositional and diagenetic fabrics are overprinted in some locations by weak to moderate shearing that forms small-scale crenulation folds with axial-planar cleavage. This type of deformation is observed in cuttings fragments from throughout the depth interval from $320 \mathrm{~m}$ ( 1050 $\mathrm{ft}$ ) to $518 \mathrm{~m}$ ( $1700 \mathrm{ft}$ ), although it appears to be especially widespread below $503 \mathrm{~m}(1650 \mathrm{ft})$. All deformational fabrics are locally crosscut by variably oriented brittle veins filled with coarse-grained, well-crystallized quartz, calcite, and brown, pleochroic carbonate rhombs (siderite-ankerite?). These youngest veins appear to post-date all significant deformation in the rock. 
The depth interval between about $521 \mathrm{~m}$ and $558 \mathrm{~m}$ (1710 ft and $1830 \mathrm{ft})$ produced cuttings that are a complex mixture of fine-grained Eleana Formation siltstone and light- to medium-gray dolomite, in part due to hole stability problems encountered at this depth (Drellack et al., 1991). The gamma-ray geophysical log indicates that dolomite was present between about 521 and $549 \mathrm{~m}$ (1710 $\mathrm{ft}$ and $1800 \mathrm{ft}$ ) (Pawloski and Carlson, 1992), and the conodonts recovered from the samples taken at $534 \mathrm{~m}$ and $549 \mathrm{~m}(1750 \mathrm{ft}$ and $1800 \mathrm{ft})$ contained distinctive fauna from the upper Simonson Dolomite or lower Guilmette Formation (Givetian-Frasnian stages; Table 29). Microscopic study of the dolostone cuttings at $534 \mathrm{~m}$ and $549 \mathrm{~m}(1750$ and $1800 \mathrm{ft})$ show intensely shattered fabrics that are cross-cut by quartz veins. On the basis of all observations, it appears that the 521-558 $\mathrm{m}$ (1710-1830 ft) zone represents a shattered slab of Middle to Late Devonian dolostone that is bounded by major faults above and below.

Beginning at about $558 \mathrm{~m}(1830 \mathrm{ft})$ and continuing to about $875 \mathrm{~m}(2870 \mathrm{ft})$, the ER-12-1 drillhole returned to fine grained-siliceous siltstone of the Mississippian upper Eleana Formation. These siltstones differ considerably from the finer-grained, laminated calcareous siltstones in the higher interval 320 to $521 \mathrm{~m}$ ( $1050 \mathrm{ft}$ to $1710 \mathrm{ft})$ ) in that they contain a higher proportion of detrital framework grains (less matrix), less calcite cement, more clasts of chert and sparse clasts of quartzite and mafic volcanic rock, and some detrital plagioclase and tourmaline. Bedding is generally thicker than the dimension of the cuttings, and the Schlumberger Formation Micro-scanner log suggests that depositional bedsets are on the order of one to three $m$ (several feet to ten feet) thick in this part of the Eleana. The composition, bedding, and textural character of these siltstones indicate they were deposited in deeper water than the fine, calcareous silts above and were transported by more turbulent submarine flow. All features are consistent with the upper Unit "J" of the Eleana Formation, but probably at a lower stratigraphic level than the Eleana encountered higher in the hole.

Deformation in this lower Eleana Formation zone is far less common than higher in the hole. These coarser siltstones show less prominent compaction foliation (because they have less matrix clay), and crosscutting shears and veins are far less common.

At about $875.0 \mathrm{~m}$ ( $2870 \mathrm{ft})$ [ $(874.7 \mathrm{~m}$ ( $2869 \mathrm{ft}$;) Schlumberger Formation Micro-scanner log)], the well penetrated a faulted contact between the Eleana and light-gray dolostone of the Middle Devonian lower Simonson Dolomite (Table 28). This dolostone is fairly uniform down to $1086 \mathrm{~m}$ (3564 ft), and shows slight variations in color, grain size, and extent of secondary silica. Breccia fabrics, veins, and other indications of deformation are sparse throughout the interval. Conodonts recovered from this zone indicate an orderly progression to older beds downward, with the top of

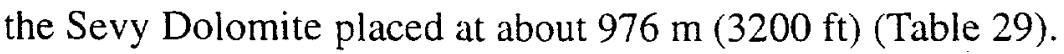

The bottom $7.3 \mathrm{~m}$ ( $24 \mathrm{ft}$ ) of well ER-12-1 [1086 to $1094 \mathrm{~m}$ (3564 to $3588 \mathrm{ft})$ ] penetrated an altered biotite-rich microporphyritic igneous rock that is interpreted to intrude the Sevy Dolomite. Detailed microscopic study of this rock by R.G. Warren (Los Alamos National Laboratory; written communication, January 1993) indicates that the primary minerals consisted of plagioclase (about 22 percent), biotite (about 20 percent), and accessory magnetite and apatite (about 1 percent each) in a fine-grained matrix. All of the matrix is replaced by chlorite, albite, sphene, and calcite, original 
calcic plagioclase has all been replaced by albite and calcite, and secondary chlorite, rutile, and sphene occur within the primary biotite. The relict mineralogy and the apparent bulk composition suggest that the original rock was probably a lamprophyre (a class of small volume intrusive igneous rocks characterized by mafic-alkalic compositions, sparse to absent quartz, and abundant mafic minerals).

Generally similar, altered, biotite-rich intrusive rocks have been observed in outcrop in several locations within the Eleana Range and in Banded Mountain in the form of thin, subvertical dikes. In three of five occurrences, the dikes were emplaced in roughly east-west orientations. If the lamprophyric intrusion encountered at the bottom of ER-12-1 is related to these mafic dikes that crop out in the area, then it would not be expected to be voluminous, and that conclusion is supported by the absence of a detectable aeromagnetic anomaly near the drill site.

Biotite was separated from cuttings obtained at the bottom of ER-12-1 and sent to the USGS Geochronology Laboratory in Menlo Park, California, for analysis by M.A. Lanphere. Despite the altered character of this mineral, the incremental-heating ${ }^{40} \mathrm{Ar}-{ }^{39} \mathrm{Ar}$ analysis showed that more than 84 percent of the argon produced between $650^{\circ} \mathrm{C}$ and $1090^{\circ} \mathrm{C}$ had a very uniform composition that indicates a weighted mean plateau age of $102.3 \pm 0.5 \mathrm{Ma}$. This age (middle Cretaceous) reflects the time of intrusion of the lamprophyre into the Sevy Dolomite (Cole et al., 1993), and is statistically equivalent to the emplacement ages previously determined for the Gold Meadows and Climax granitic stocks (Naeser and Maldonado, 1981).

\section{Thermal History of Rocks at the ER-12-1 Well}

Conodonts that were recovered from the carbonate rocks in ER-12-1 were also examined to determine the thermal history of the site. Conodonts systematically change color as they are exposed to increasing temperature, either as a result of sedimentary burial, metamorphism, or intrusion. The Color Alteration Index (CAI) (Epstein et al., 1977) has been calibrated to an absolute temperature scale and can provide a measure of the maximum temperature imposed on the sample over its total history. In addition, the presence of mixed-CAI-value conodonts in an individual sample, indicating exposure to non-equilibrium thermal conditions, has been shown to correlate with hydrothermal alteration and contact metamorphic geologic settings (Epstein et al., 1977).

The CAI values recorded by conodonts at various depths in ER-12-1 are listed in Table 30. The values of 4-4.5 to 5.5 are typical of the Devonian and lower Paleozoic rocks in this part of southern Nevada and probably reflect the combined effects of sedimentary burial, thrusting during the Antler and Sevier orogenies, and regional heating during Cenozoic extension (Harris et al., 1980). Superimposed on this background, however, is the overprint of a non-uniform heating event whose effect is progressively greater toward the bottom of the hole. Numerous samples produced variable conodont CAI values that indicate a local or short-lived heat source, and many conodonts in ER-12-1 show etched and pitted surfaces due to the corrosive effect of hydrothermal fluids (A. Harris, written communication, 1992).

Six samples of the Eleana siltstone from ER-12-1 were also analyzed for hydrocarbon content

by C. Barker in the Branch of Petroleum Geology, USGS, Denver. Progressive heating in the 
Table 30. Conodont Color Alteration Index (CAI) Values.

\begin{tabular}{|c|c|c|c|}
\hline Depth $\mathrm{m}(\mathrm{ft})$ & CAI Range & $\begin{array}{l}\text { Maximum Rock } \\
\text { Temperature }\end{array}$ & Comment \\
\hline $\begin{array}{l}0-137 \mathrm{~m} \\
(0-450 \mathrm{ft})\end{array}$ & $\begin{array}{l}4-4.5 \\
\text { some } 5.5\end{array}$ & $\begin{array}{l}>220^{\circ} \mathrm{C} \\
\text { up to } 320\end{array}$ & $\begin{array}{l}\text { Hydrothermally altered; Sevy } \\
\text { Dolomite, early Early Devonian }\end{array}$ \\
\hline $\begin{array}{l}152-244 \mathrm{~m} \\
(500-800 \mathrm{ft})\end{array}$ & $5-5.5$ & $>320^{\circ} \mathrm{C}$ & Sevy Dolomite; Late Silurian \\
\hline $\begin{array}{l}259-274 \mathrm{~m} \\
(850-900 \mathrm{ft})\end{array}$ & $5-5.5$ & $>320^{\circ} \mathrm{C}$ & $\begin{array}{l}\text { Antelope Valley Limestone; } \\
\text { Middle Ordovician }\end{array}$ \\
\hline $\begin{array}{l}289-320 \mathrm{~m} \\
(950-1050 \mathrm{ft})\end{array}$ & $5-5.5$ & $>320^{\circ} \mathrm{C}$ & Sevy Dolomite; Late Silurian \\
\hline $\begin{array}{l}533-549 \mathrm{~m} \\
(1750-1800 \mathrm{ft})\end{array}$ & $5.5-6.5$ & $>350-400{ }^{\circ} \mathrm{C}$ & $\begin{array}{l}\text { Upper Simonson Dolomite or } \\
\text { lower Guilmette Formation; late } \\
\text { Middle Devonian; maximum } \\
\text { temperatures probably } \\
\text { somewhat higher than } 400{ }^{\circ} \mathrm{C}\end{array}$ \\
\hline $\begin{array}{l}869-945 \mathrm{~m} \\
(2850-3100 \mathrm{ft})\end{array}$ & $\begin{array}{l}\text { some } 5 ; 5.5-6.5 \\
\text { some } 7\end{array}$ & $\begin{array}{l}>300^{\circ} \mathrm{C} \\
\text { up to } 450^{\circ} \mathrm{C}\end{array}$ & $\begin{array}{l}\text { Hydrothermally altered; } \\
\text { uppermost Sevy Dolomite or } \\
\text { lowest Simonson Dolomite; late } \\
\text { Early Devonian and Middle } \\
\text { Devonian }\end{array}$ \\
\hline $\begin{array}{l}970-1006 \mathrm{~m} \\
(3180-3300 \mathrm{ft})\end{array}$ & $\begin{array}{l}\text { some } 5 ; 5.5-6.5 \\
\text { some } 7\end{array}$ & $\begin{array}{l}>300^{\circ} \mathrm{C} \\
\text { up to } 450^{\circ} \mathrm{C}\end{array}$ & $\begin{array}{l}\text { Hydrothermally altered; Sevy } \\
\text { Dolomite; Early Devonian }\end{array}$ \\
\hline $\begin{array}{l}1021-1082 \mathrm{~m} \\
(3350-3550 \mathrm{ft})\end{array}$ & mixed 5 through 7 & $\begin{array}{l}>300^{\circ} \mathrm{C} \\
\text { up to } 4500^{\circ} \mathrm{C}\end{array}$ & $\begin{array}{l}\text { Hydrothermally altered; Sevy } \\
\text { Dolomite; Late Silurian to Early } \\
\text { Devonian }\end{array}$ \\
\hline
\end{tabular}

(Data from A. G. Harris, USGS, Reston, VA; written communication, 1992) 
pyrolysis technique produced no volatile hydrocarbons and confirmed that the Eleana has been subjected to maximum temperatures on the same order as those indicated by the CAI values (greater than $350^{\circ} \mathrm{C}$ ).

The combined results of the conodont CAI analyses and the rock pyrolysis analyses indicate that the carbonate rocks in ER-12-1, as well as the intervening sheets of Eleana siltstone, have been thermally overprinted following movement on the faults that separate them. The probable source of heat for this thermal disturbance is the microporphyritic intrusion encountered at the bottom of the hole, and its age establishes that the major fault activity must have occurred prior to $102.3+0.5$ Ma (middle Cretaceous).

\section{GEOPHYSICAL LOGS}

The logging suite for ER-12-1 was chosen by a committee, consisting of representatives of the Department of Energy, Desert Research Institute, Lawrence Livermore National Laboratory (LLNL), Los Alamos National Laboratory (LANL), Raytheon Services Nevada, and the United States Geological Survey, during the planning stages of the hole. A broad range of disciplines and interests were represented, e.g., drillers, logging engineers, geophysicists, geologists, hydrologists, and administrators. As a result, the range of logging techniques requested was quite broad.

Many of the logs run in ER-12-1 were experimental to the logging community on the NTS. On-site logging capability (at the time ER-12-1 was drilled) was optimized for logging in very large ( 2.18 to $3.05 \mathrm{~m}$ or 86 to 120 in diameter) holes, in predominantly very porous, yet unsaturated rocks, with the holes filled with either air or water/mud. By comparison, well ER-12-1 had a small radius, so dry-hole logging tools, typically used on the NTS, were too large to fit into the well. Industry-standard logging capabilities, driven by the needs of oil and gas exploration, are optimized for holes slightly smaller ( 0.10 to $0.30 \mathrm{~m}$ or 4 to 12 in diameter) than ER-12-1. However, the response of many of these logs in air-filled holes is undocumented.

Standard logging technology was chosen for ER-12-1 as the best fit for the environment of the $0.31 \mathrm{~m}$ ( $12.25 \mathrm{in}$ ) diameter, water-filled portion of the borehole. In addition, many of these logs were available through the former on-site logging subcontractor (Atlas Wireline Services). The following section describes each log, the processing history, special comments on each data type, and provides preliminary correlation with geological and hydrological data.

\section{Geophysical Logging}

Downhole geophysical logs were obtained by Atlas Wireline Services, Schlumberger, Westech Engineering (video camera), and LLNL NTS Geotechnical Engineering Group. Table 31 lists all logs acquired in ER-12-1 to date. The LLNL gravimeter was run in the ER-12-1 borehole following the installation of the $0.14 \mathrm{~m}$ (5.5 in) completion string, once the drilling rig was no longer present at the surface.

The subcontract under which Atlas Wireline conducted logging at the NTS referred to a manual

of logging procedures that described in detail how the work was to be conducted (Atlas, 1988). This 
Table 31. List of Geophysical Logs Acquired in ER-12-1.

\begin{tabular}{|c|c|c|c|c|c|c|c|c|c|}
\hline Hole & Log Type & Run \# & Date & Logger & Drldep & Logde & $\begin{array}{l}\text { Toplog } \\
\text { Meters }\end{array}$ & 3otlog Wa & \\
\hline ER-12-1 & $3-D^{1}$ & 1 & 19-Oct-1991 & ATLAS WS & 1093.6 & 1091.5 & 460.2 & 1083.3 & - \\
\hline ER-12-1 & CALIPER & 1 & 20-Aug-1991 & ATLAS WS & 520.9 & 517.6 & 1.2 & 515.1 & - \\
\hline ER-12-1 & CALIPER & 3 & 26-Oct-1991 & ATLAS WS & 1093.6 & 1065.0 & 433.4 & 1062.5 & - \\
\hline ER-12-1 & CALIPER & 4 & 29-Oct-1991 & ATLAS WS & 1093.6 & 633.1 & 430.7 & 630.3 & - \\
\hline ER-12-1 & CALIPER & 5 & 12-Nov-1991 & ATLAS WS & 1093.6 & 549.2 & 432.8 & 546.5 & . \\
\hline ER-12-1 & CALIPER+ & 2 & 18-Oct-1991 & ATLAS WS & 1093.6 & 1091.5 & 435.6 & 1088.4 & 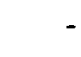 \\
\hline ER-12-1 & $\mathrm{CVL}^{2}$ & 1 & 19-Oct-1991 & ATLAS WS & 1093.6 & 1091.5 & 460.2 & 1085.7 & 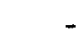 \\
\hline ER-12-1 & DENSITYBC 3 & 1 & 20-Aug-1991 & ATLAS WS & 520.9 & 516.0 & 7.0 & 515.1 & . \\
\hline ER-12-1 & DENSITYBC & +2 & 19-Oct-1991 & ATLAS WS & 1093.6 & 1091.5 & 435.9 & 1090.6 & \\
\hline ER-12-1 & DIP MICRO 4 & 1 & 22-Oct-1991 & SCLUMBRGR & 1093.6 & 1092.1 & 449.3 & 1091.8 & - \\
\hline ER-12-1 & DIR SEEK $^{5}$ & 1 & 17-Jan-1992 & EASTMAN & 1093.6 & - & 7.6 & 1073.5 & 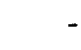 \\
\hline ER-12-1 & ELOG IND 6 & 1 & 21-Aug-1991 & ATLAS WS & 520.9 & 516.3 & 10.7 & 515.4 & - \\
\hline ER-12-1 & ELOG MINI $^{7}$ & 1 & 22-Oct-1991 & SCLUMBRGR & 1093.6 & 1091.2 & 449.3 & 1087.5 & - \\
\hline ER-12-1 & ELOGFOCUS ${ }^{8}{ }^{8}$ & 1 & 20-Oct-1991 & ATLAS WS & 1093.6 & 1091.5 & 442.0 & 1088.1 & 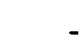 \\
\hline ER-12-1 & GAMMA SPEC $^{9}$ & 1 & 20-Aug-1991 & ATLAS WS & 520.9 & 516.3 & 0.6 & 515.7 & \\
\hline ER-12-1 & GAMMA SPEC & 2 & 18-Oct-1991 & ATLAS WS & 1093.6 & 1091.5 & 436.5 & 1091.2 & 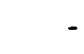 \\
\hline ER-12-1 & GAMMA+ $+^{10}$ & 1 & 20-Aug-1991 & ATLAS WS & 520.9 & 516.3 & 9.1 & 511.5 & . \\
\hline ER-12-1 & GAMMA+ & 2 & 18-Oct-1991 & ATLAS WS & 1093.6 & 1091.5 & 435.6 & 1088.4 & - \\
\hline ER-12-1 & GAMMA+ & 3 & 19-Oct-1991 & ATLAS WS & 1093.6 & 1091.5 & 431.0 & 1090.0 & - \\
\hline ER-12-1 & GAMMA+ & 4 & 19-Oct-1991 & ATLAS WS & 1093.6 & 1091.5 & 435.9 & 1090.6 & - \\
\hline ER-12-1 & GAMMA+ & 5 & 20-Oct-1991 & ATLAS WS & 1093.6 & 1091.5 & 442.0 & 1088.1 & . \\
\hline ER-12-1 & MAGNET BH ${ }^{l l}$ & 1 & 21-Aug-1991 & LLNL-N & 520.9 & 517.2 & 21.3 & 517.2 & - \\
\hline ER-12-1 & MAGNET BH & 2 & 21-Oct-1991 & LLNL-N & 1093.6 & 1092.4 & 452.0 & 1092.4 & - \\
\hline ER-12-1 & $\mathrm{NCTL}^{12}$ & 1 & 23-Aug-1991 & ATLAS WS & 520.9 & 448.7 & 0.6 & 448.4 & - \\
\hline ER-12-1 & NEUTRONEP +13 & ${ }^{3} 1$ & 20-Aug-1991 & ATLAS WS & 520.9 & 516.3 & 9.1 & 515.1 & - \\
\hline ER-12-1 & NEUTRONEP+ & 2 & 19-Oct-1991 & ATLAS WS & 1093.6 & 1091.5 & 431.0 & 1090.0 & - \\
\hline ER-12-1 & SEISMIC DN ${ }^{14}$ & 1 & 19-Oct-1991 & ATLAS WS & 1093.6 & 1088.4 & 91.4 & 1088.4 & - \\
\hline ER-12-1 & VIDEO & 1 & 21-Aug-1991 & WESTECH & 520.9 & - & 0.0 & 467.0 & - \\
\hline ER-12-1 & WL FLD ${ }^{15}$ & 1 & 20-Aug-1991 & ATLAS WS & 520.9 & 520.6 & 455.4 & 490.7 & 468.8 \\
\hline ER-12-1 & WL FLD & 2 & 21-Aug-1991 & ATLAS WS & 520.9 & - & 459.3 & 479.1 & 468.8 \\
\hline ER-12-1 & WL FLD & 3 & 22-Aug-1991 & ATLAS WS & 520.9 & 515.7 & 442.0 & 475.5 & 449.9 \\
\hline \multirow[t]{2}{*}{ ER-12-1 } & WL FLD & 4 & 18-Oct-1991 & ATLAS WS & 1093.6 & - & 455.1 & 476.7 & 470.9 \\
\hline & TOTAL: & & & & & & & & \\
\hline
\end{tabular}

${ }^{1}$ Acoustic fracture log

${ }^{2}$ Compensated velocity

${ }^{3}$ Borehole compensated density

${ }^{4}$ Formation Micro-scanner

${ }^{5}$ Gyroscopic survey
${ }^{6}$ Induction $\log$

${ }^{7}$ Micro-resistivity log

${ }^{8}$ Focused resistivity

${ }^{9}$ Spectral gamma ray

${ }^{10}$ Gamma ray
"'Borehole magnetometer

${ }^{12}$ Nuclear cement toplog

${ }^{13}$ Epithermal neutron (ENP) or (Neutron EP)

${ }^{14}$ Downhole seismic

${ }^{15}$ Water level 
manual was written principally by LLNL, published by RSN, and agreed to by LLNL, LANL, RSN, and Atlas Wireline. Services not expressly described in this manual were run under logging company standard procedures, and supplemented by specific instructions at the well site. This operating procedure applied to the Schlumberger and Westech logging performed at ER-12-1. Specific NTS procedures existed for the epithermal neutron, gamma ray, and temperature (ENP, GR, and TL) logs. The LLNL logging procedures were defined in a separate document (Millet and Felske, 1989), which had been reviewed and accepted by the LLNL Containment Program.

Connection of signal processing elements to the logging cable, the selection of what data was to be presented on film and tape, and the format of that data was controlled by the logging truck computer via utilization of a service table. A service table is defined as a small file of information set up for each log and called up by the computer when that particular log was to be run. Much of the previously discussed NTS procedure consisted of descriptions of the specific service table to be used for each log.

An attempt was made to decrease the time spent logging at ER-12-1 by stacking logging tools. Stacked logging tools consists of connecting several mutually compatible tools end-to-end and recording all data during one run in the borehole. This is a common practice in the logging industry, and Atlas Wireline had copies of the requisite service tables for Gamma Ray (GR)/Compensated Density Log (CDL)/Dual Induction Focused Log (DIFL); Six Arm Caliper Log (CA6)/GR; and ENP/GR stacks. All of the stacks were tried, but most would not work, though much time and effort were applied in the attempt. Sondes and equipment, brought to the location by Atlas Wireline, were apparently different than those used in typical oil field applications. In the dry hole, only the ENP/GR stack worked. Additional work by Atlas Wireline, conducted between August 21, 1991 (when the dry-hole logs were run) and October 18,1991 (when the wet-hole logs were run) resulted in successful logging of the wet-hole with the ENP/GR, CA6/GR, CDL/GR and Dual Laterolog (DLL)/GR stacks. The stacking of these logs did not result in saved time as all of the logs were also run separately. The stacked logs did, however, provide valuable depth correlation between the runs owing to the multiple gamma ray logs. It should be noted that the GR trace was strongly affected by the CDL source, and to a lesser extent by the ENP source, in the dry hole runs. The gamma ray data from these stacked configurations should be used qualitatively, but not quantitatively.

LLNL personnel and RSN logging engineers were present for the Atlas dry-and wet-hole logging suites conducted at ER-12-1. Their presence provided a technical resource for decisions regarding data quality and initial data interpretation.

Logging tools were required to pass quality control standards prior to being run in well ER-12-1. However, some of the tools did not have calibrations or gap corrections for processing data from the $0.44 \mathrm{~m}(17-1 / 2 \mathrm{in})$ diameter, air-filled environment of the upper portion of ER-12-1. In response, LLNL developed new processing procedures for these logs, and has worked with the DOE in an attempt to correct these deficiencies for future drill holes.

One item needs to be noted; casing depths on all logs appeared to be approximately $2 \mathrm{~m}(6 \mathrm{ft})$ shallower than the drilling report. Investigations indicated the logging data were consistent; it is 
suspected that the pipe count was wrong. Additionally, the video log shows the surface casing base at $15.33 \mathrm{~m}$ (50.3 ft), which was consistent with neither logging information (14.02 $\mathrm{m}$ or $46 \mathrm{ft})$ nor drilling information $(15.85 \mathrm{~m}$ or $52 \mathrm{ft})$.

\section{Presentation of Data}

Figures 5 and 6 summarize data types that were common to the dry and wet logging suites. Processed caliper, bulk density, water content, resistivity, natural gamma, and total magnetic intensity are shown, with stratigraphy supplied by RSN geologists and modified on the basis of paleontological investigations. At the bottom of each log trace is the log name and the date it was run. Water content is displayed as percent volume, which readily equates with porosity.

Table 32 lists processed ER-12-1 data archived by the LLNL Containment Program. The processed data have also been transferred to DRI. The data given to DRI have been smoothed on $0.61 \mathrm{~m}(2 \mathrm{ft})$ intervals, and then tabulated on $0.30 \mathrm{~m}(1 \mathrm{ft})$ increments. This slightly reduced the large number of data points in each file, while making it easier to display and manipulate the information with CAD software. Data at this sampling rate should be sufficient for other uses, such as modeling.

Strict quality control was employed throughout the processing of the data at LLNL. All codes were documented, and history files were constructed which include information from the actual processing procedure. These history files are part of the QA record, and are archived at LLNL.

\section{Caliper Logs}

Five caliper logs were run in ER-12-1. A 6-arm caliper tool was utilized, where each arm represented a radius, and diameters were identified by paired arms, i.e., diameters 1-4, 2-5, and 3-6. Caliper Run 2 was stacked with a gamma tool, and both tools acquired information as they were pulled together up the drill hole. Caliper Run 1 (shown in Figure 5) was run to the intermediate depth of $520.90 \mathrm{~m}(1709 \mathrm{ft})$ and obtained information predominantly in the dry portion of the hole $(52.12$ $\mathrm{m}$ or $171 \mathrm{ft}$ of data were obtained below the static water level from this run). Runs 2 through 5 were

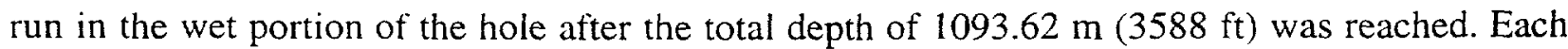
caliper log started as close to the bottom of the hole as possible.

Caliper processing included determining the minimum and maximum diameters from the three pairs of arms at all locations, the ratio of the maximum to minimum diameters (called elongation index), and the average of the three diameters at each depth. Tolerances on acquiring data were 6 $\mathrm{mm}$ (1/4 in) on each diameter. The accuracy of the average diameter was $4 \mathrm{~mm}$ (just under $1 / 4 \mathrm{in}$ ). Average diameter and the individual three measured diameters were archived. The change in bit size at $520.90 \mathrm{~m}$ ( $1709 \mathrm{ft}$ ) is visible in the data from Runs 2 through 5 (Figure 7). In general, the hole was relatively in gauge, with exceptions being from $73.15-129.54 \mathrm{~m}(240-425 \mathrm{ft})$ and $521.21-550.77$ $\mathrm{m}(1710-1810 \mathrm{ft})$.

Casing locations indicated by the log trace on all five runs appeared to be about $2 \mathrm{~m}(6 \mathrm{ft})$ shallower than the drilling report. The surface casing was reported to be at $15.85 \mathrm{~m}(52 \mathrm{ft})$, but

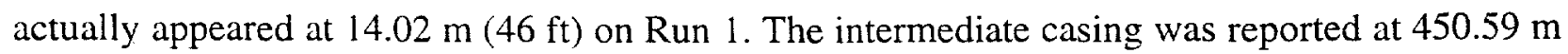
(1478 ft), but appeared at $448.66 \mathrm{~m}(1472 \mathrm{ft})$ on Runs 2-5. 


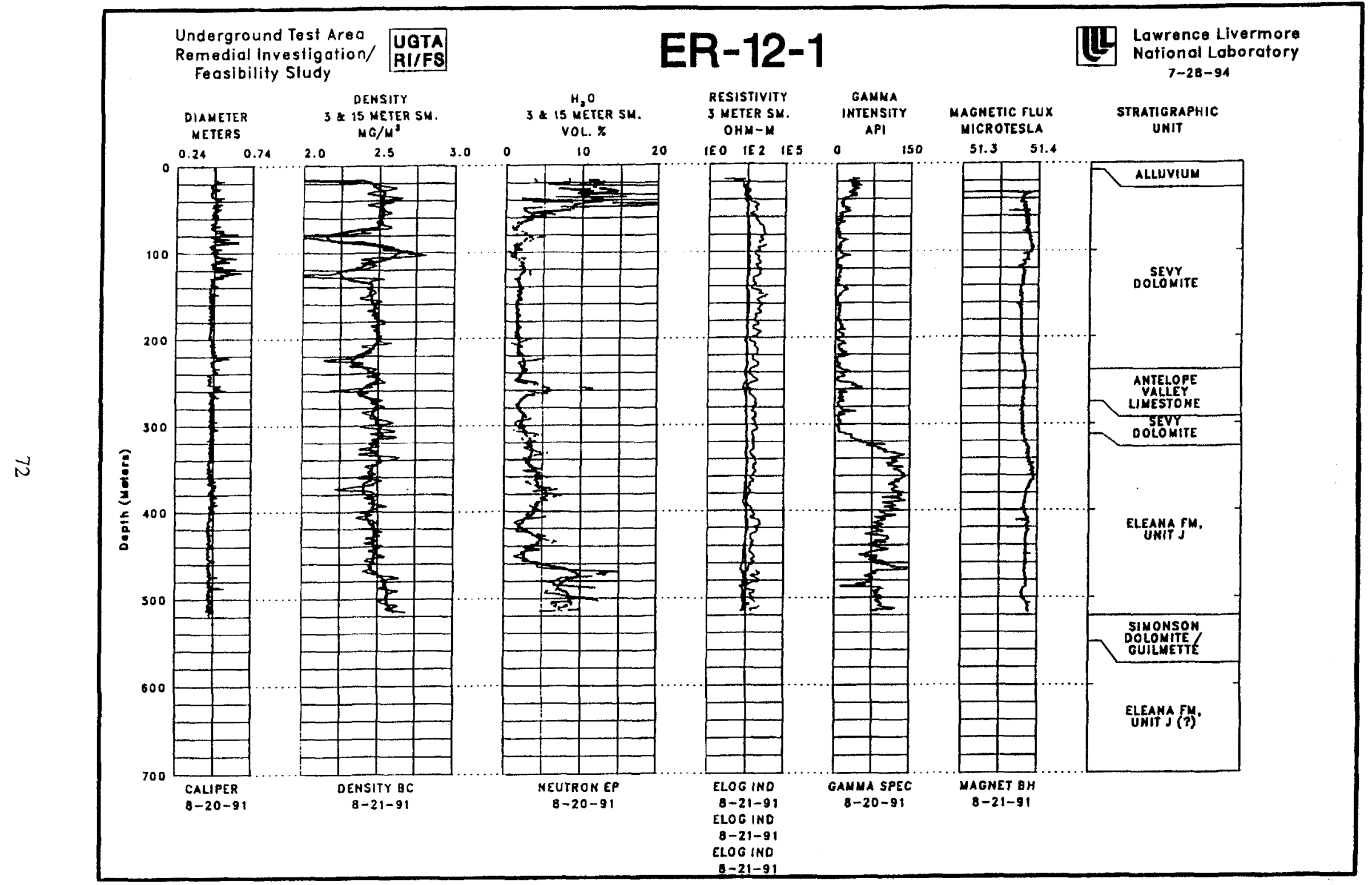

Figure 5. Summary of selected logs from the dry-hole logging suite. 


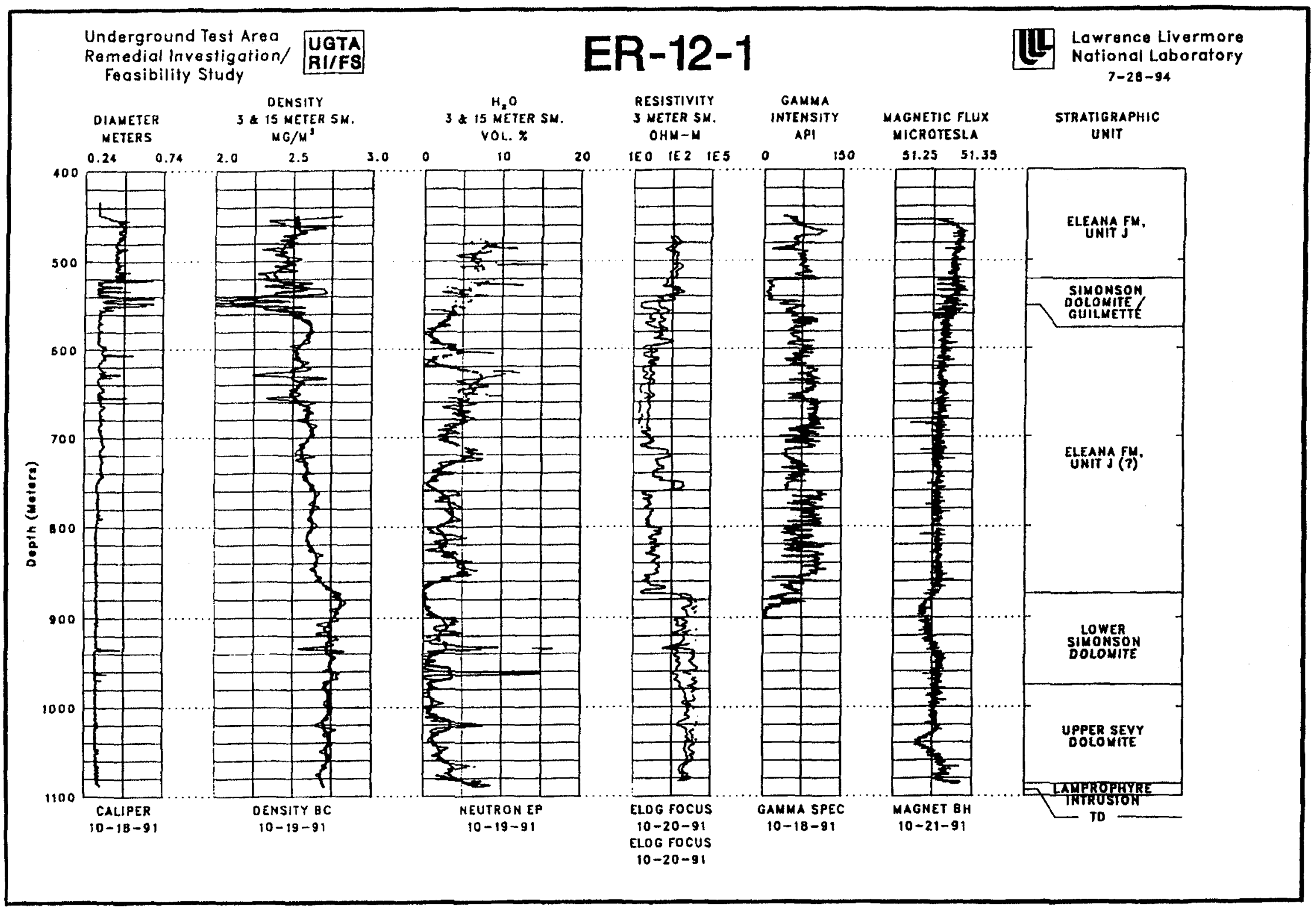

Figure 6. Summary of selected logs from the wet-hole logging suite. 
Table 32. Processed Data from ER-12-1 Archived at LLNL.

\begin{tabular}{|c|c|c|c|c|c|c|c|c|c|c|c|}
\hline $\begin{array}{l}\text { Hole } \\
\text { Name } \\
\end{array}$ & $\begin{array}{c}\text { Log } \\
\text { Name } \\
\end{array}$ & $\begin{array}{c}\text { Run } \\
\#\end{array}$ & $\begin{array}{l}\text { Log } \\
\text { Date } \\
\end{array}$ & $\begin{array}{l}\text { Data } \\
\text { Type }\end{array}$ & Units & $\begin{array}{l}\text { Number } \\
\text { of Points }\end{array}$ & $\begin{array}{l}\text { Min. } \\
\text { Value }\end{array}$ & $\begin{array}{l}\text { Max. } \\
\text { Value }\end{array}$ & $\begin{array}{l}\text { Min. } \\
\text { Depth }\end{array}$ & $\begin{array}{l}\text { Max. } \\
\text { Depth }\end{array}$ & $\begin{array}{l}\text { Archived } \\
\text { File Name } \\
\end{array}$ \\
\hline ER-12-1 & CALIPER & 1 & 20-aug-1991 & DIAMETER & INCHES & 6816 & 17.41 & 26.58 & -3.8 & 1700.0 & VO5D10F71 \\
\hline$E R-12-1$ & CALIPER & 1 & 20-aug-199] & DIAMETER I & INCHES & 6816 & 16.53 & 29.80 & -3.8 & 1700.0 & Vo5D10F74 \\
\hline$E R-12-1$ & CALIPER & 1 & 20-aug-199! & DIAMETER 2 & INCHES & 6816 & 16.89 & 28.88 & -3.8 & 1700.0 & VO5DI0F75 \\
\hline$E R-12-1$ & CALIPER & 1 & 20-aug-1991 & DIAMETER 3 & INCHES & 6816 & 16.74 & 33.01 & -3.8 & 1700.0 & VO5D10F76 \\
\hline ER-12-1 & CALIPER & 2 & 18-oct-1991 & DIAMETER & INCHES & 8601 & 11.90 & 26.94 & 1421.0 & 3571.0 & VO5D11F13 \\
\hline ER-12-1 & CALIPER & 2 & $18-$ oct-1991 & DIAMETER 1 & INCHES & 8601 & 11.29 & 33.08 & 1421.0 & 3571.0 & VO5D11F14 \\
\hline ER-12-1 & CALIPER & 2 & 18-oct-1991 & DIAMETER 2 & INCHES & 8601 & 11.19 & 28.29 & 1421.0 & 3571.0 & VO5DIJF15 \\
\hline ER-12-1 & CALIPER & 2 & $18-o c t-1991$ & DIAMETER 3 & INCHES & 8601 & 11.66 & 31.16 & 1421.0 & 3571.0 & VOSD1IF16 \\
\hline ER-12-1 & CALIPER & 3 & $26-$ oct-1991 & DIAMETER & INCHES & 8253 & 12.18 & 28.76 & 1422.0 & 3485.0 & VOSD1IF17 \\
\hline ER-12-1 & CALIPER & 3 & $26-0 c t-1991$ & DIAMETER I & INCHES & 8253 & 11.95 & 33.94 & 1422.0 & 3485.0 & VO5DIIF18 \\
\hline$E R-12-1$ & CALIPER & 3 & 26-oct-1991 & DIAMETER 2 & INCHES & 8253 & 11.50 & 28.67 & 1422.0 & 3485.0 & VO5DIIF19 \\
\hline ER-12-1 & CALIPER & 3 & $26-\mathrm{oct}-1991$ & DIAMETER 3 & INCHES & 8253 & 11.87 & 30.45 & 1422.0 & 3485.0 & VOSDIIF20 \\
\hline ER-12-1 & CALIPER & 4 & $29-$ oct-199! & DIAMETER & INCHES & 2593 & 12.63 & 30.67 & 1420.0 & 2068.0 & VO5D11F31 \\
\hline ER-12-1 & CALIPER & 4 & $29-$ oct-1991 & DIAMETER 1 & INCHES & 2593 & 12.63 & 33.71 & 1420.0 & 2068.0 & VOSD11F32 \\
\hline ER-12-1 & CALIPER & 4 & 29-oct-1991 & DIAMETER 2 & INCHES & 2593 & 12.42 & 28.97 & 1420.0 & 2068.0 & VOSD11F33 \\
\hline ER-12-1 & CALIPER & 4 & $29-0 \mathrm{cl}-1991$ & DIAMETER 3 & INCHES & 2593 & 12.63 & 30.63 & 1420.0 & 2068.0 & VO5D1IF34 \\
\hline ER-12-1 & CALIPER & 5 & 12-nov--1991 & DIAMETER & INCHES & 1525 & 12.62 & 28.21 & 1412.0 & 1793.0 & VOSD11F35 \\
\hline ER-12-1 & CALIPER & 5 & 12-nov-1991 & DIAMETER I & INCHES & 1525 & 12.74 & 31.86 & 1412.0 & 1793.0 & V05D11F36 \\
\hline$E R-12-1$ & CALIPER & 5 & 12-nov-1991 & DIAMETER 2 & INCHES & 1525 & 12.46 & 30.21 & 1412.0 & 1793.0 & V05D11F37 \\
\hline$E R-12-1$ & CALIPER & 5 & 12-nov-1991 & DIAMETER 3 & INCHES & 1525 & 12.37 & 29.72 & 1412.0 & 1793.0 & VO5D11F38 \\
\hline ER-12-1 & CVL & 1 & 19-oct-1991 & VELOCITY & FT/SEC & 8041 & 12504.50 & 24966.19 & 1550.0 & 3560.0 & VO5D1IF39 \\
\hline ER-12-1 & DENSITY BC & 1 & $21-a u g-1991$ & DENSITY & $\mathrm{GM} / \mathrm{CC}$ & 6561 & 1.37 & 3.02 & 52.0 & 1692.0 & VOSD11F45 \\
\hline ER-12-1 & DENSITY BC & 2 & 19-oct-1991 & DENSITY & $\mathrm{GM} / \mathrm{CC}$ & 8405 & 1.29 & 3.04 & 1475.0 & 3576.0 & VOSD1IF46 \\
\hline ER-12-1 & ELOG FOCUS & 1 & 20-oct-1991 & RESISTIVITY & OHM-M & 8025 & 2.09 & 19933.46 & 1550.0 & 3556.0 & VO5D11F52 \\
\hline$E R-12-1$ & ELOG FOCUS & 1 & $20-0 \mathrm{ct}-1991$ & RESISTIVITY & OHM-M & 8025 & 2.00 & 19647.56 & 1550.0 & 3556.0 & V05D11F53 \\
\hline ER-12-1 & ELOG FOCUS & 1 & 20-oct-1991 & SELF-POTENTIAL & MILLIVOLTS & 7885 & -249.71 & 194.00 & 1550.0 & 3521.0 & V05D11F54 \\
\hline$E R-12-1$ & ELOG IND & 1 & $21-$ aug-1991 & RESISTIVIVITY & OHM-M & 6720 & 172.80 & 5904.77 & 11.3 & 1691.0 & VO5D10F68 \\
\hline$E R-12-1$ & ELOG IND & 1 & 21 -aug-1991 & RESISTIVIVITY & $\mathrm{OHM}-\mathrm{M}$ & 6720 & 2.57 & 9809.16 & 11.3 & 1691.0 & VO5D10F69 \\
\hline ER-12-1 & ELOG IND & 1 & 21-aug-1991 & RESISTIVIVITY & OHM-M & 6720 & 5.21 & 514.54 & 11.3 & 1691.0 & V05D10F70 \\
\hline ER-12-I & GAMMA SPEC & 1 & 20-aug-1991 & GAMMA & API UNITS & 6557 & 1.02 & 147.74 & 52.0 & 1691.0 & VO5D11F21 \\
\hline ER-12-1 & GAMMA SPEC & 1 & 20-aug-1991 & POTASSIUM & VOL-PERCNT & 6557 & 0.00 & 4.98 & 52.0 & 1691.0 & VOSD11F22 \\
\hline $\mathrm{ER}-12-1$ & GAMMA SPEC & 1 & 20-aug-199! & URANIUM & PPM & 6557 & 0.41 & 5.82 & 52.0 & 1691.0 & VO5D11F23 \\
\hline$E R-12-1$ & GAMMA SPEC & 1 & 20-aug-1991 & THORIUM & PPM & 6557 & 0.15 & 12.94 & 52.0 & 1691.0 & VO5D11F24 \\
\hline ER-12-1 & GAMMA SPEC & 2 & $18-o c t-1991$ & GAMMA & API UNITS & 5929 & 1.52 & 122.52 & 1478.0 & 2960.0 & VO5D11F40 \\
\hline ER-12-1 & GAMMA SPEC & 2 & 18-oct-1991 & POTASSIUM & VOL-PERCNT & 5929 & -0.01 & 3.64 & 1478.0 & 2960.0 & VOSDIIF41. \\
\hline ER-12-1 & GAMMA SPEC & 2 & 18 -oct-1991 & URANIUM & PPM & 5929 & 0.25 & 7.99 & 1478.0 & 2960.0 & VO5D11F42 \\
\hline ER-12-1 & GAMMA SPEC & 2 & 18-oct-1991 & THORIUM & PPM & 5929 & 0.66 & 12.71 & 1478.0 & 2960.0 & VOSD11F43 \\
\hline$E R-12-1$ & MAGNET BH & 1 & 21-aug-1991 & MAGNETIC FLUX DENS & MICROTESLA & 1700 & 51.35 & 52.69 & 70.8 & 1696.3 & VO5D11F49 \\
\hline ER-12-1 & MAGNET BH & 2 & $21-$ oct-1991 & MAGNETIC FLUX DENS & MICROTESLA & 2693 & 51.25 & 51.35 & 1483.0 & 3584.6 & VO5DIIF58 \\
\hline ER-12-1 & NEUTRON EP & 1 & 20-aug-1991 & WATER CONTENT & WT-PERCENT & 3276 & -0.13 & 17.69 & 52.0 & 1689.5 & VO5D11F47 \\
\hline$E R-12-1$ & NEUTRON EP & 1 & 20-aug-1991 & WATER CONTENT & VOL-PERCENT & 3276 & -0.38 & 45.03 & 52.0 & 1689.5 & VO5D11F48 \\
\hline ER-12-1 & NEUTRON EP & 2 & 19-oct-1991 & WATER CONTENT & WT-PERCENT & 4063 & -0.39 & 19.80 & 1544.0 & 3575.0 & VO5D11F29 \\
\hline ER-12-1 & NEUTRON EP & 2 & 19-oct-1991 & WATER CONTENT & VOL-PERCNT & 4063 & -1.04 & 38.90 & 1544.0 & 3575.0 & VOSD11F30 \\
\hline ER-12-1 & SEISMIC DN & 1 & 19-oct-1991 & VELOCITY & FT/SEC & 47 & 8285.71 & 15687.22 & 0.0 & 3571.0 & V05D11F25 \\
\hline ER-12-1 & SEISMIC DN & 1 & $19-o c t-1991$ & TRAVEL TIME & SEC & 47 & 0.04 & 0.23 & 0.0 & 3571.0 & VO5D11F26 \\
\hline
\end{tabular}




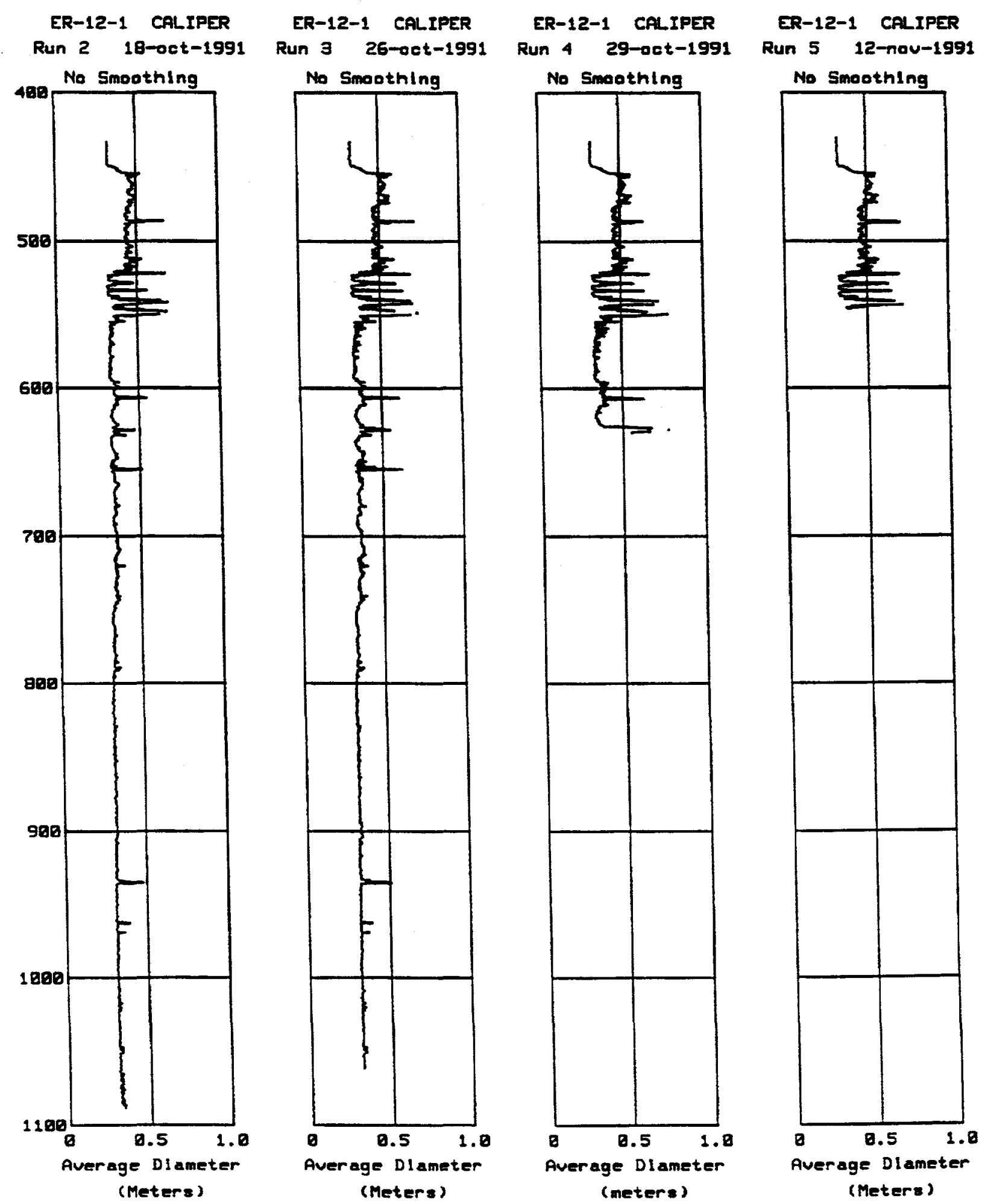

Figure 7. ER-12-1 caliper logs, 400 to $1100 \mathrm{~m}$ (1312 to $3608 \mathrm{ft})$. 
Data from Runs 2 and 3 were very repeatable, with both showing the hole being in-gauge for most of the depth, with minor washouts. The repeatability indicated that the hole was fairly stable at this point. Both runs showed a ragged interval between 521.21 to $550.77 \mathrm{~m}$ (1710 to $1807 \mathrm{ft}$ ) in the Simonson Dolomite. There were five enlarged zones within this interval. Four zones correlated to faults identified in the hole, and the remaining zone correlated to a fractured zone. Total depth of the hole changed between Run 2 (1091.49 $\mathrm{m}$ or $3581 \mathrm{ft}$ ) and Run 3 ( $1064.97 \mathrm{~m}$ or $3494 \mathrm{ft}$ ). Other logs run within days of Caliper Run 2 showed a tagged depth of near $1090 \mathrm{~m}$ ( $3576 \mathrm{ft}$ ), indicating sloughing occurred after October 21, 1991 (Magnetometer Run 2) but before October 26, 1991 (Caliper Run 3). Since the data obtained by Caliper Runs 2 and 3 are so similar, the sloughing was probably restricted to the bottom of the hole. Initial well development began sometime after Caliper Run 3, when bentonite drilling fluid was removed from the well and replaced with fresh water. This caused hole instability. Caliper Run 4 on October 29, 1991 tagged fill high in the hole at $633.07 \mathrm{~m}$ $(2077 \mathrm{ft})$, indicating that the hole had probably bridged. The rocks at this location were argillaceous bedded units within the Eleana Formation (Unit J). Run 5, on November 12, 1991, showed an even shallower total depth ( $549.25 \mathrm{~m}$ or $1802 \mathrm{ft}$ ), indicating hole stability still had not been reestablished.

Faults and fractures identified by RSN geologists using stratigraphy, lithology, geophysical logs, and drilling data were, in general, quite visible on the caliper logs above $603 \mathrm{~m}$ (1978 ft). Several sharp breaks in the caliper logs were indicated between 603 to $840 \mathrm{~m}$ (1978 to $2756 \mathrm{ft}$ ), but none of these were identified by the geologists as faults or fractures. The caliper logs were relatively smooth between 850 to $915 \mathrm{~m}$ ( 2790 to $3000 \mathrm{ft}$ ) where faults and fracture have been identified. Between 930 to $965 \mathrm{~m}$ (3050-3170 ft) faults and fractures correlated with peaks on the caliper logs.

\section{Density Logs}

Two compensated density logs (CDLs), which are two-detector tools, were run in ER-12-1; Run 1 was part of the dry hole suite, and Run 2 was part of the wet suite. Run 2 was stacked with a gamma log. Data from compensated density logs were used to: determine the bulk density of the rock media, assist in lithologic correlation, and calculate physical properties of saturation and porosity.

No calibration data existed for a two-detector tool run in a $0.44 \mathrm{~m}$ (17.5 in) diameter air-filled hole. Under water, the tool responded properly to the environment, and data processing was guided by standard procedures. A review of the Atlas Wireline corrected bulk density and the correction factor utilized revealed that the corrected density values appeared to be approximately $0.1-0.2$ $\mathrm{Mg} / \mathrm{m}^{3}$ lower than expected for the rock types encountered. In addition, the short-spaced density was higher than the long-spaced density, which is physically impossible for a properly calibrated tool run in a borehole caked with mud having a density less than the formation density. In addition, the automatic correction was negative, which was also not physically possible under these conditions. Atlas Wireline routinely made their compensation correction to the density curve utilizing constants determined in wet environments at their Houston test pits. This configuration was not appropriate for air-filled drill holes at the NTS. LLNL recognized this inconsistency, and subsequently developed methods to resolve the problem. 
Below the water level, the tool responded appropriately to the environment (water-filled gaps rather than air-filled gaps), and processing of the data was straightforward. Negative correction was not observed and the short-spaced count was always less than or equal to the long-spaced count. Previous experience in these environments encouraged confidence that the tool was operating correctly. The measured densities were as expected for the given rock type and matched laboratory-measured gravimetric density data. Water levels used in processing data from both runs

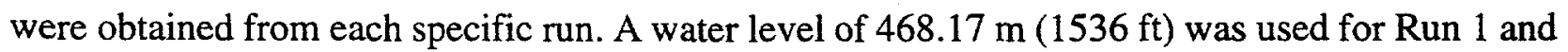
$470.61 \mathrm{~m}$ (1544 ft) was used for processing Run 2.

The density data were processed using the long- and short-spaced count data. First the count rates from the two detectors were converted to density by applying the calibration densities to the counts on the log, which converted the counts from saturated limestone bulk density to electron density in the process. A least squares technique was used in a running window on the two log densities to determine the standoff correction to be applied to the long-spaced density. In the wet hole, this process gave densities that agreed within $0.02 \mathrm{Mg} / \mathrm{m}^{3}$ with electron densities calculated from the field density curve. In the dry hole, the long-spaced density values were increased and the short-spaced values decreased until the two curves agreed at their high density excursions, and the step in density at the static water level was minimized. The final calibration density values used were $1.756 \mathrm{Mg} / \mathrm{m}^{3}$ and $2.795 \mathrm{Mg} / \mathrm{m}^{3}$ for the long-spaced values, and $1.696 \mathrm{Mg} / \mathrm{m}^{3}$ and $2.625 \mathrm{Mg} / \mathrm{m}^{3}$ for the short. The unshifted electron density values were $1.628 \mathrm{Mg} / \mathrm{m}^{3}$ and $2.685 \mathrm{Mg} / \mathrm{m}^{3}$ for both long and short, so the long-spaced density was shifted by about $0.1 \mathrm{Mg} / \mathrm{m}^{3}$ and the short-spaced density by about $0.06 \mathrm{Mg} / \mathrm{m}^{3}$. The step at the SWL was reduced from about $0.2 \mathrm{Mg} / \mathrm{m}^{3}$ to about $0.05 \mathrm{Mg} / \mathrm{m}^{3}$.

Two bulk density measurements were made on core samples taken at $754.38 \mathrm{~m} \mathrm{(2475} \mathrm{ft)}$ (Drellack et al., 1991). The measured values of 2.68 and $2.67 \mathrm{Mg} / \mathrm{m}^{3}$ compared favorably to the $\mathrm{log}$ data from Run 2.

Density data from ER-12-1 are shown in Figures 8 and 9 along with water content and velocity data. The accuracy of the processed data was different (for reasons explained above) for the dry and wet portions of the hole. There was a high level of confidence that the reported density data below the water level were accurate to $0.063 \mathrm{Mg} / \mathrm{m}^{3}$. A lower level of confidence existed in the data from the dry portion of the hole, and therefore, an accuracy of $0.1 \mathrm{Mg} / \mathrm{m}^{3}$ was reported for data from 15.85 to $468.26 \mathrm{~m}$ ( 52 to $1536 \mathrm{ft}$ ) from Run 1 .

\section{Epithermal Neutron Logs}

Two epithermal neutron logs (ENP) were run in ER-12-1, both in a stacked configuration with gamma ray tools. Run 1 was part of the dry suite of logs, Run 2 part of the wet suite. Water content was the main data type determined from the measurements, however, the data can also be used with other data to determine porosity, saturation, and lithology. Hydrogen Contact Test Unit (HCTU) calibration data were used for processing data from both runs. The calibration data applied to 0.31 $\mathrm{m}$ (12.25 in) diameter holes in both wet and dry conditions. Calibration data for $0.44 \mathrm{~m}$ (17.5 in) diameter holes were not available. 
Calibrations to determine the multiplier to convert count data (CPS) to API units on Run 1 were not conducted according to standard practice, however, the calibration procedure that was used resulted in a difference of only 0.16 percent, thus the log data were considered acceptable. The repeat section was used as it had better quality data than that in the main run. A fluid level of 467.87 $\mathrm{m}(1535 \mathrm{ft})$ was used for processing, taken directly from this log run rather than from a fluid density measurement of $468.78 \mathrm{~m}(1538 \mathrm{ft}$ ) made earlier in the day. Density data from compensated density $\log$ Run 1 were used to apply the density correction to the water data. There was a significant increase in water content below the water level, as was expected.

Run 2 was processed using density data from compensated density $\log$ Run 2 , and a water level of $470.61 \mathrm{~m}$ ( $1544 \mathrm{ft}$ ), which was obtained from the trace of the log.

Plots of water content from ER-12-1 are displayed in Figures 8 and 9, along with bulk density and velocity data. The accuracy of the volumetric water content was estimated at \pm 8 percent of the value. Since the density correction was small for calculating volumetric water content, the error was assumed to be the same for both the dry and wet sections.

\section{Electric Logs}

Four different electric logs were run to obtain lithologic information (altered zones) and physical property information (porosity and saturation). Electric logs do not perform properly in casing, so data from all runs were collected below the cased interval. A dual induction focused log (DIFL) was run as part of the dry hole suite. A Dual Laterolog (DLL), Schlumberger Microlog, and Schlumberger Formation Micro-scanner (FMS) were included as part of the wet hole suite. Each of these logs are discussed below.

\section{Dual Induction Focused Log (Elog Ind)}

The dual induction focused $\log$, which was run as part of the dry suite of logs, recorded three types of resistivity information - deep, medium, and focused. The medium and deep data were measured as conductivity, by virtue of the physics of the method. The accuracy of the log was 2 mmho-m. However, at a resistivity of $200 \mathrm{ohm}-\mathrm{m}$ (the realm of a fair amount of the data from ER-12-1, after it is converted from conductivity to resistivity), this value resulted in an accuracy of approximately $40 \%$ of the value. There were no tool geometry factors available in the logging industry for correcting data run in a $0.44 \mathrm{~m}$ (12.5 in) diameter hole, so corrections were not made to these data. Resistivity data from the DIFL were off-scale much of the time, due in part to a small error in conductivity zero. This was corrected during processing by adding a constant to each data trace to prevent conductivity from crossing zero. The medium conductivity trace was corrected by $1.0 \mathrm{mmho} / \mathrm{m}$, deep conductivity was corrected by $-0.676 \mathrm{mmho} / \mathrm{m}$, and the focused conductivity trace was corrected by $-0.880 \mathrm{mmho} / \mathrm{m}$. Conductivity was then converted to resistivity, and anomalous spikes were stripped out.

Resistivity data from this log are shown in Figure 5, but they are displayed in a clearer form in Figure 10. The deep resistivity trace was lower than the medium resistivity trace as the medium 


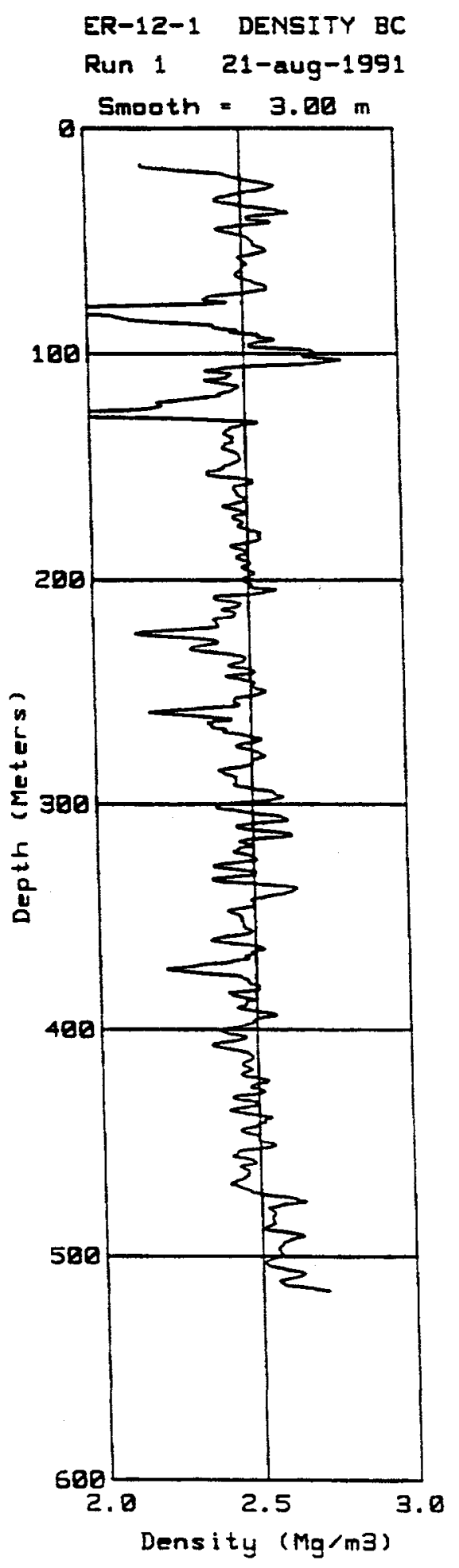

ER-12-1 NEUTRON EP
Run 1 2Q-aug-1991

Smooth $=3.00 \mathrm{~m}$

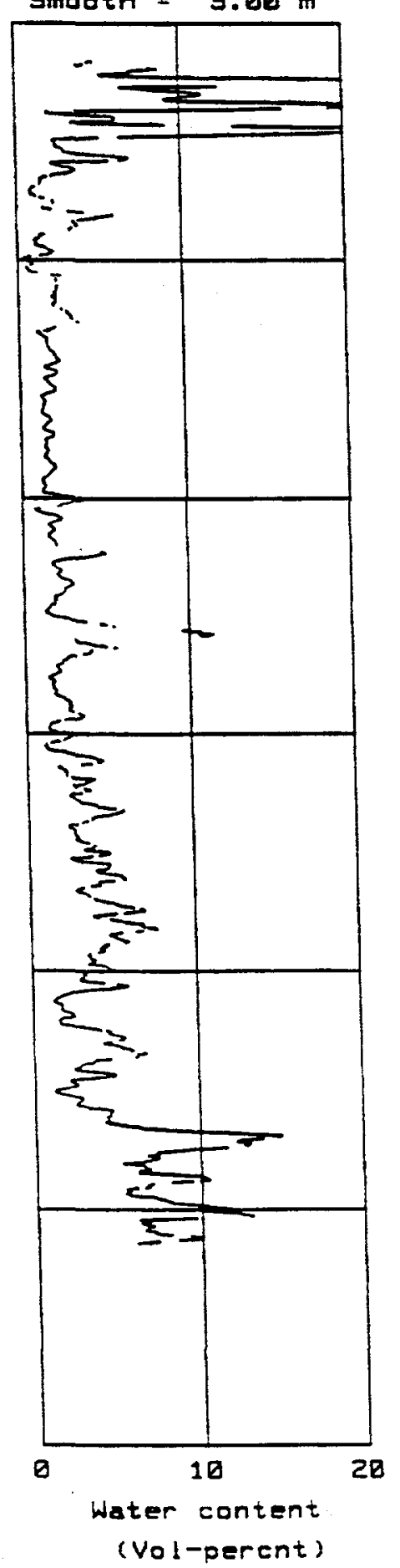

ER-12-1 SEISMIC DN

Run 1 19-act-1991

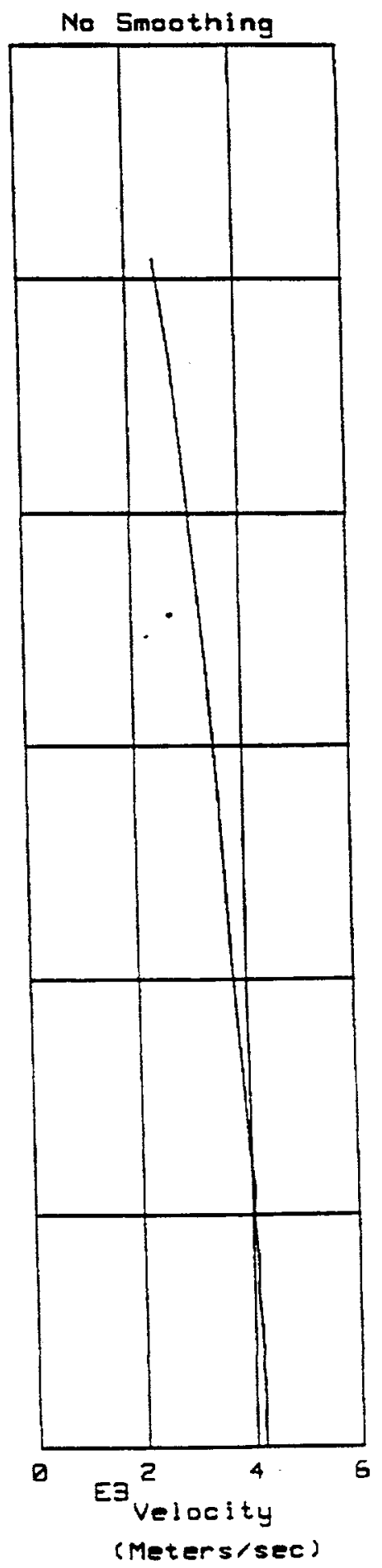

Figure 8. Density, epithermal neutron, and sonic logs from ER-12-1,0 to $600 \mathrm{~m}$ (0 to $1968 \mathrm{ft}$ ). 

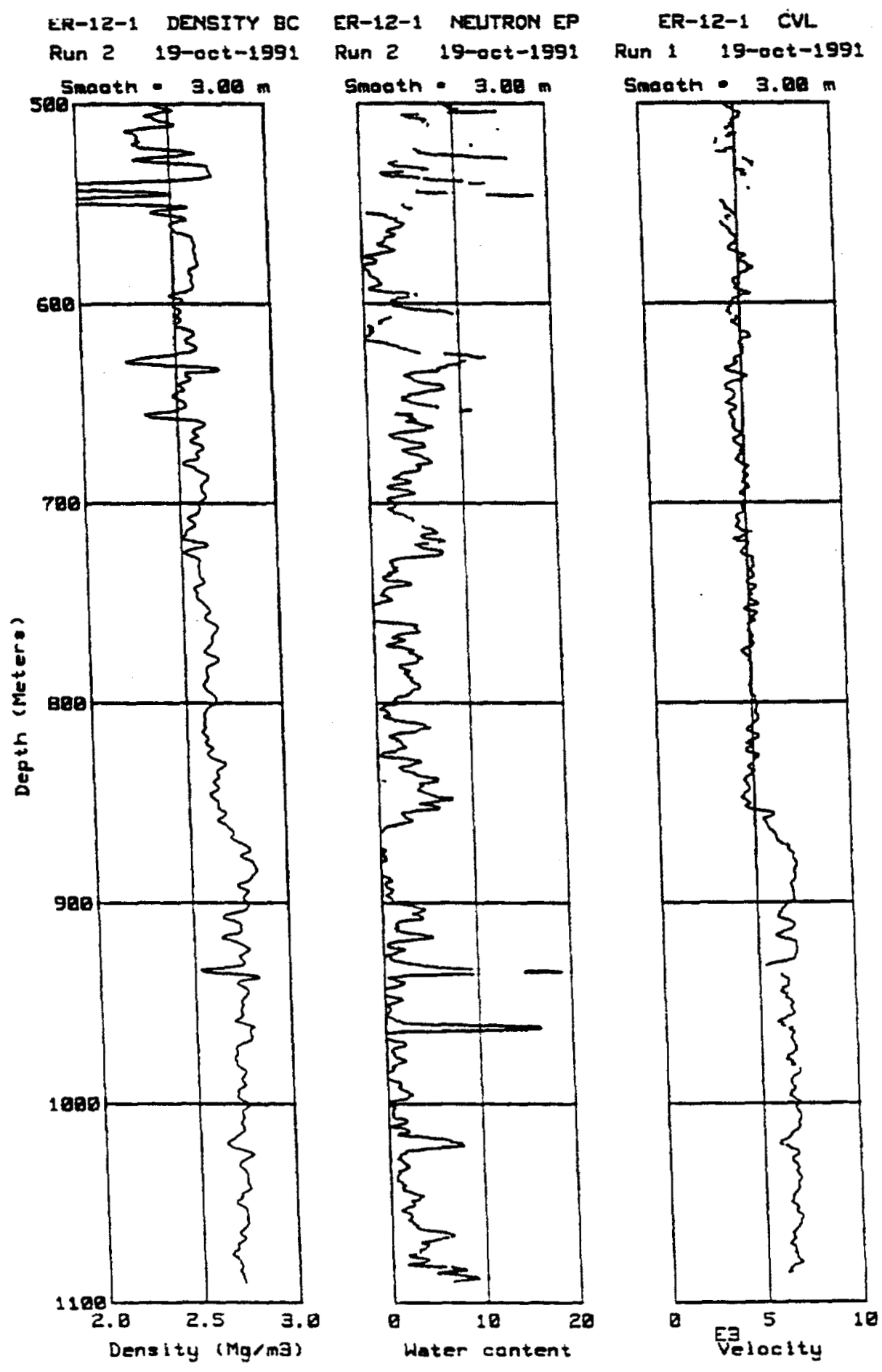

ER-12-1 SEISMIC DN

Smooth - $3.08 \mathrm{~m}$

Smath - $3.09 \mathrm{~m}$

un 1 19-0et-1991
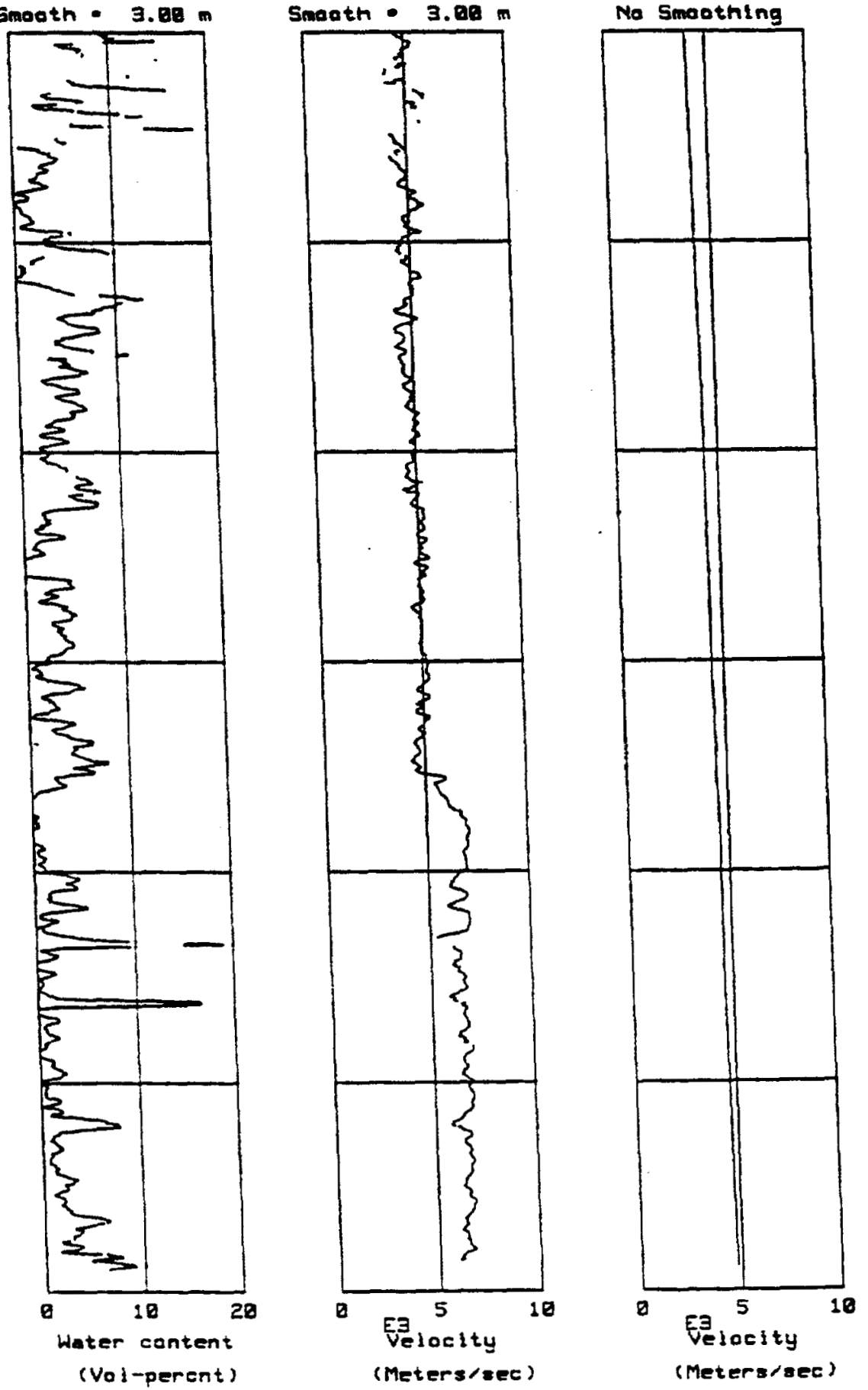

Figure 9. Density, epithermal neutron, continuous velocity and sonic $\log , 500$ to $1100 \mathrm{~m}$ (1640 to $3608 \mathrm{ft}$ ). 


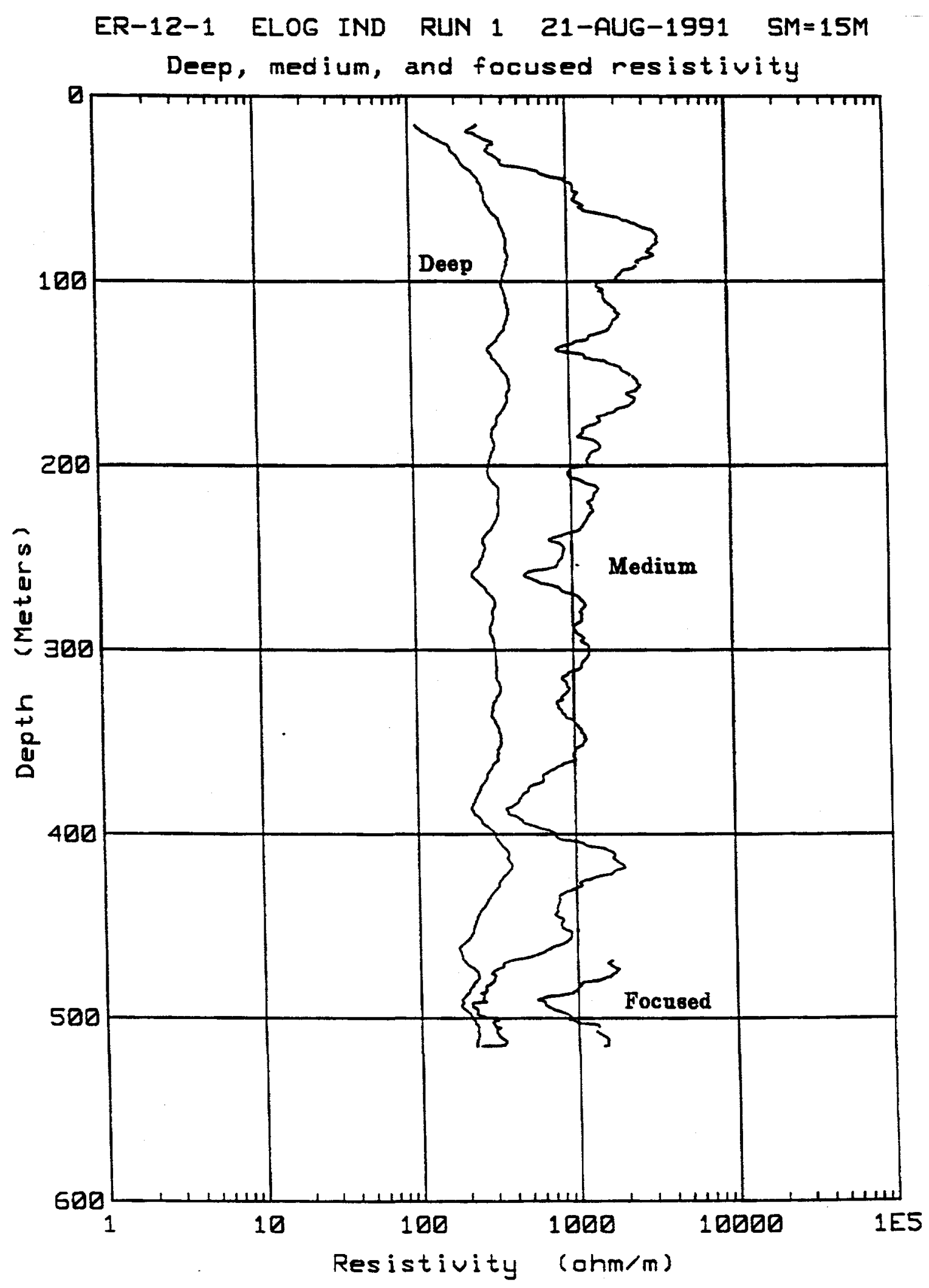

Figure 10. Deep, medium and focused resistivity, 0 to $600 \mathrm{~m}(0$ to $1968 \mathrm{ft}$ ). 
trace was affected by the air-filled borehole. The two traces were in better agreement below the SWL. The focused data were collected only below the SWL. Values from this trace were higher, falling between the shallow and deep curves of the DLL.

\section{Dual Laterolog (Elog Focus)}

The dual laterolog is a wet hole tool that measures deep and shallow resistivity and spontaneous potential (SP). It was run in a stacked configuration with Gamma Run 5. Since the electric log data were acquired in a water-filled hole, tool geometry was not an influential factor. Atlas Wireline Services noted under remarks for this log that spikes on the resistivity curves in high resistivity zones were due to software problems. Processing included removing anomalous spikes from both resistivity files by stripping at limits determined by flat-topped data. Resistivity data are shown in Figures 6 and 11, and spontaneous potential data are presented in Figure 12. It is clear in Figures 6 and 11 that the changes in deep and shallow resistivity with depth were not identical; at times they were far apart, and at times they were coincidental. Separation of the traces usually indicated a zone of lower resistivity near the borehole that has experienced shallow invasion by drilling fluids.

\section{Microlog (Elog Mini)}

A Microlog is a wet-hole tool that records diameter, natural gamma, and resistivity, and can be useful in determining mudcake or invaded zone resistivity. Digital data from this log was not obtained from Schlumberger, so no data were processed by LLNL.

\section{Formation Micro-Scanner Log (Dip Micro)}

This $\log$ is an enhanced dip meter that measures resistivity, natural gamma, hole direction, and two diameters that are displayed as differences. The blueline log trace/printout obtained from Schlumberger displayed eight individual resistivity traces. All of these data were useful in determining geologic structure in the hole, i.e., dips of bedding, fractures, and faults. Large volumes of data were collected. Special processing by the logger and geologic input from the geologist were required to maximize the value of the $\log$. The results of this $\log$ were presented in Schlumberger (1992), which is archived at DRI and RSN.

\section{Gamma Logs}

\section{Spectralog}

This log measures the full spectrum of natural radiation in the drill hole. Data from the log were useful in determining lithology and stratigraphy. The data were recorded digitally as count spectra, and then reprocessed by Atlas in Houston for gain corrections. Typically, four windows of data from the total spectrum were utilized: gamma, K (potassium), U (uranium), and Th (thorium). Gamma data were the total count of the entire spectrum, converted to API units by LLNL using a conversion factor determined in support of the Weapons Program via testing the tool in a calibration facility. $\mathrm{K}, \mathrm{U}$, and Th data were converted from counts to the presented units (vol percent, ppm, and ppm, respectively) by Atlas from their standard oil field calibrations. The accuracy of these values were 


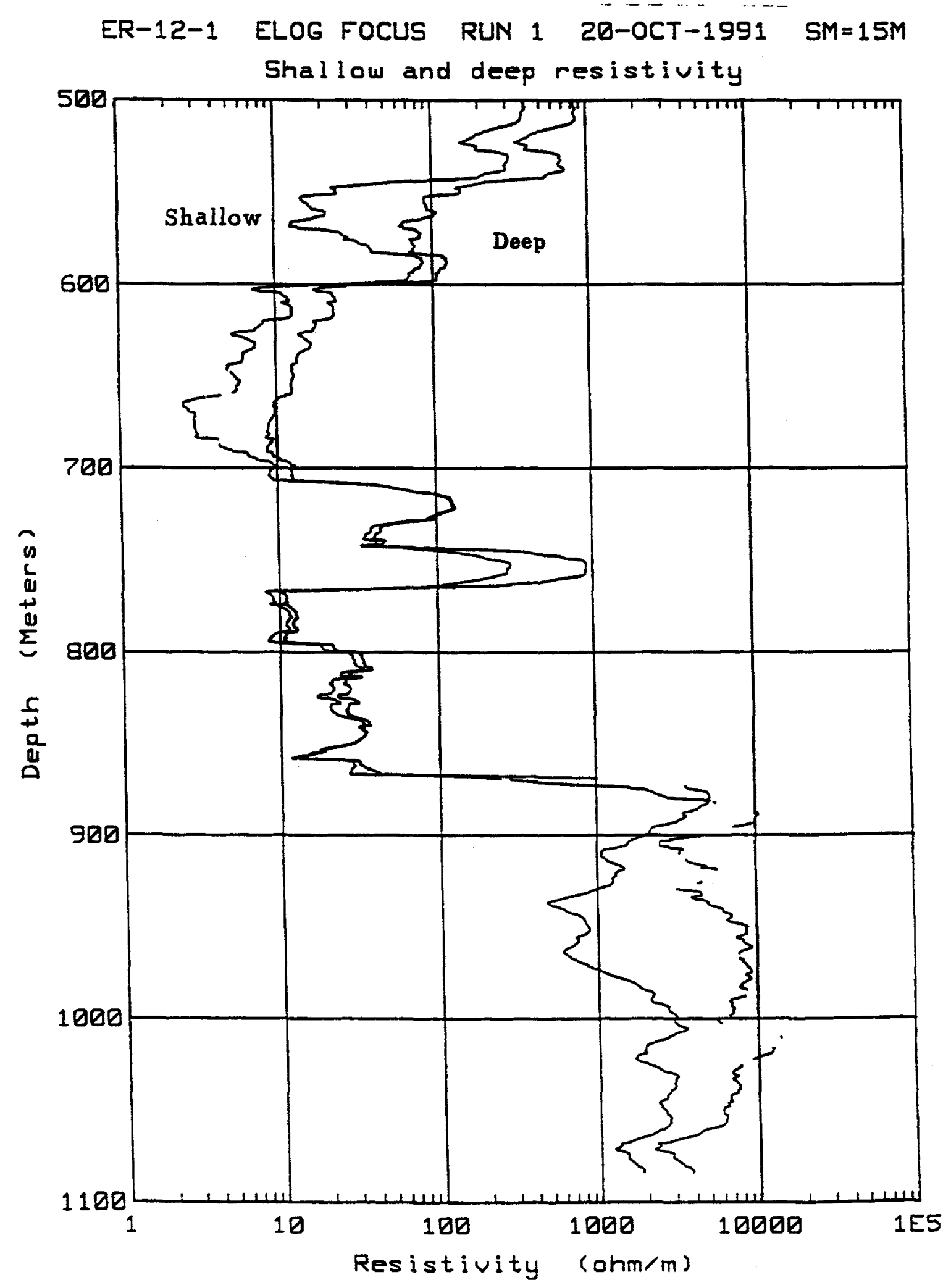

Figure 11. Shallow and deep resistivity, 500 to $1100 \mathrm{~m}$ (1640 to $3608 \mathrm{ft}$ ). 


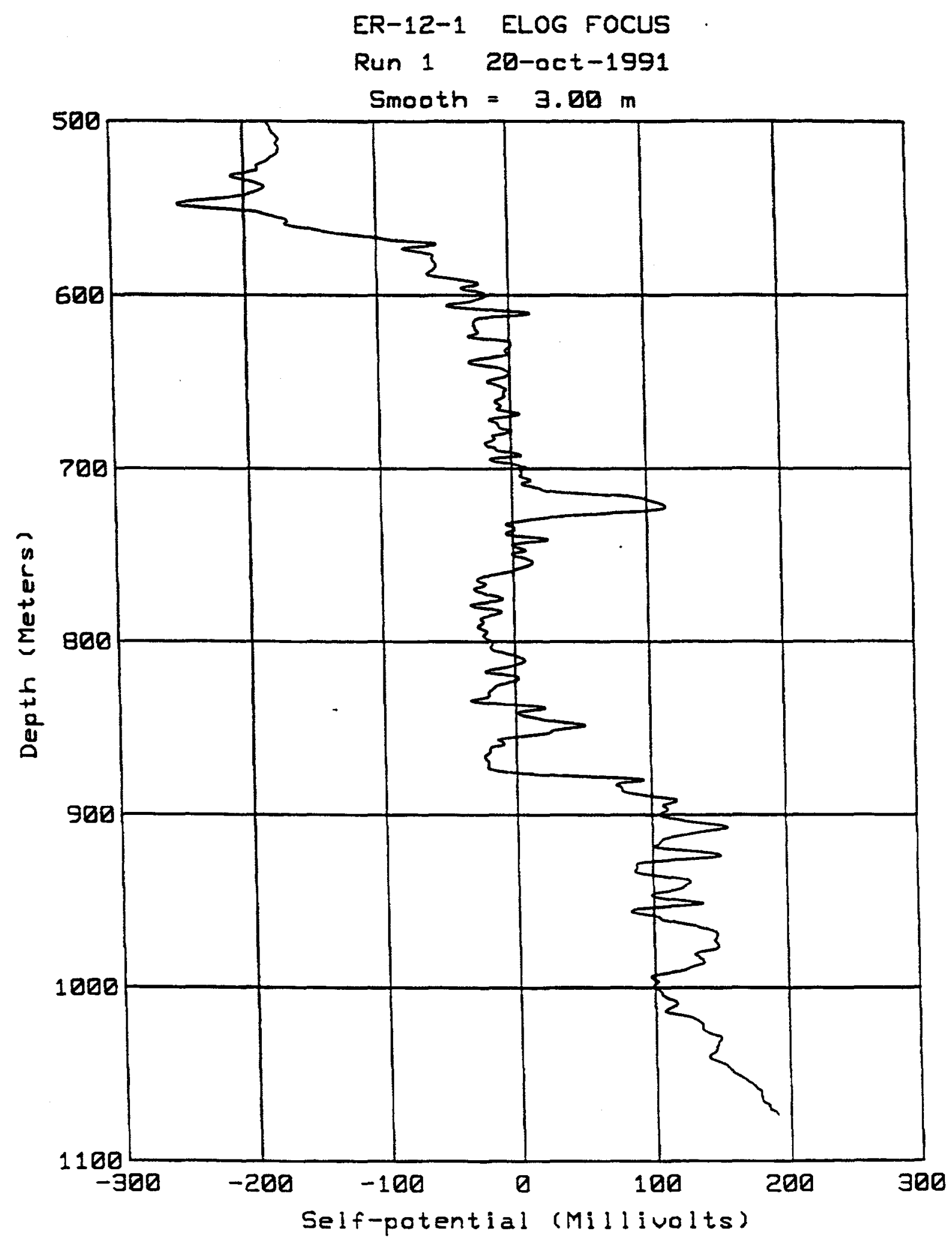

Figure 12. Self potential, 500 to $1100 \mathrm{~m}$ (1640 to $3608 \mathrm{ft})$. 
unknown. Frequently, these traces, or combinations of these traces, are used to look for potential zones of swelling clays. RSN geologists did not find correlations between the log and geologic cuttings data. Stratigraphic and lithologic contacts were shown quite well by the gamma traces in Figures 5 and 6. Figures 13 and 14 display gamma, K, U, and Th traces for both spectral gamma runs.

This log does have some deficiencies. The crystal must be warmed before going downhole. Normally, this would occur during preparation for the run, and not impinge on actual logging time. However, if something goes wrong during the warm up procedure, several hours can be added to the waiting time as the crystal correctly comes up to temperature. To obtain statistically accurate, well defined data, the tool is pulled slowly up the hole, at a much slower rate than other logging tools ( $1 \mathrm{vs} 10 \mathrm{~m} /$ minute or $3 \mathrm{vs} 30 \mathrm{ft} /$ minute). It took eight hours to gather data on Run 1 , and nine hours for the second run. However, a significant amount of geologic information was obtained from this $\log$, so it is a valuable tool to run.

\section{Gamma}

Five gamma logs were run in ER-12-1. This tool uses a much smaller crystal, and is pulled at a faster rate than the spectral gamma log discussed above. All five gamma logs were stacked with other logs; epithermal neutron run 1 , caliper run 2 , epithermal neutron run 2 , compensated density run 2 , and dual laterolog run 1 . While the data from the epithermal neutron and compensated density logs cannot be utilized quantitatively because of the interfering radiation sources, the data from all runs can be utilized qualitatively. This provided useful depth and lithology correlation. LLNL relied on the spectral gamma log for natural gamma information from ER-12-1.

\section{Total Intensity Magnetometer Logs}

A proton precession magnetometer was run by the LLNL NTS Geotechnical Engineering Group in both the dry and wet portions of the drill hole. Tool response depends on magnetic properties of the rock, e.g., susceptibility, and in the case of volcanic rocks, remnant magnetism frozen in the rock at the time of deposition or cooling. There were some concerns that data obtained from this $\log$, run in the sedimentary rocks at ER-12-1, would not offer much information, since sedimentary rocks usually are not composed of significant amount of magnetic minerals. Thus, this $\log$ was run as a test case as part of both dry and wet logging suites.

Processing of the data from magnetometer logs is straightforward as no hole size or environmental (wet/dry) corrections are necessary. For ER-12-1, however, the dry hole was affected in the upper $70 \mathrm{~m}$ (about $200 \mathrm{ft}$ ) by the drill rig at the surface. The metal in the rig was enough to perturb the down hole signal. Under normal conditions these data would have been discarded. An algorithm was developed to remove the effect of the rig from the upper portion of the data, thus saving about $70 \mathrm{~m}$ of data. The same thing happened to data from Run 2 due to the presence of the 450 - $\mathrm{m}$-long intermediate casing. The algorithm used to correct data from Run 1 was modified slightly to fit the conditions of Run 2, and the casing signal was also removed. Figures 15 and 16 show the corrected and uncorrected magnetometer data. At depths where the runs overlap, data from Run 2 reads approximately 0.05 microTeslas lower than Run 1 . This may be due to the use of different sondes in the different runs. 


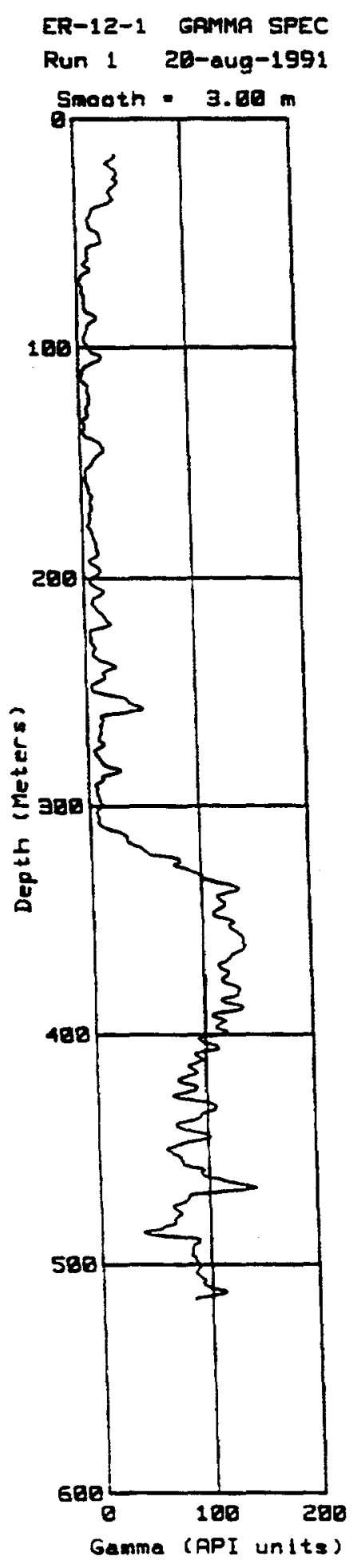

ER-12-1 GAMMA SPEC Run 1 28-aug-1991

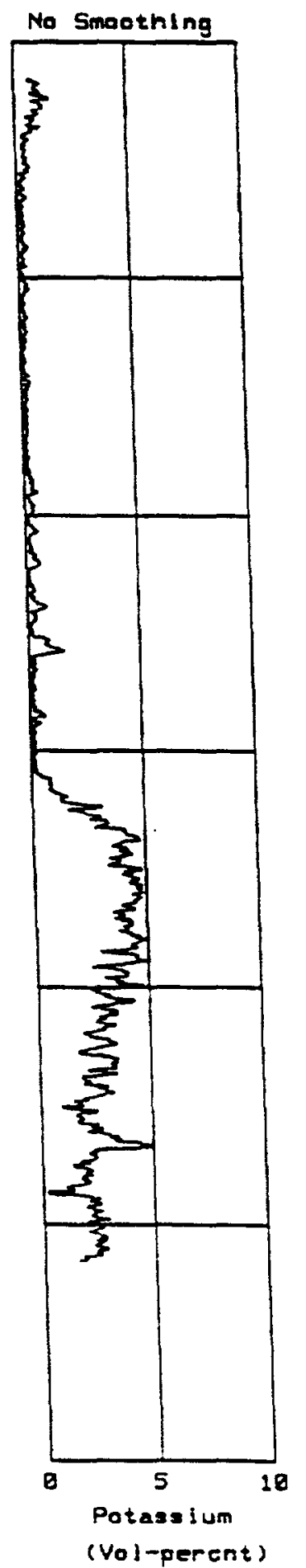

ER-12-1 GAMMA SPEC

ER-12-1 GAMA SPEC Run 1 2Q-aug-1991

Run 1 20-aug-1991

Snooth - $3.80 \mathrm{~m}$

Smooth - $3.00 \mathrm{~m}$
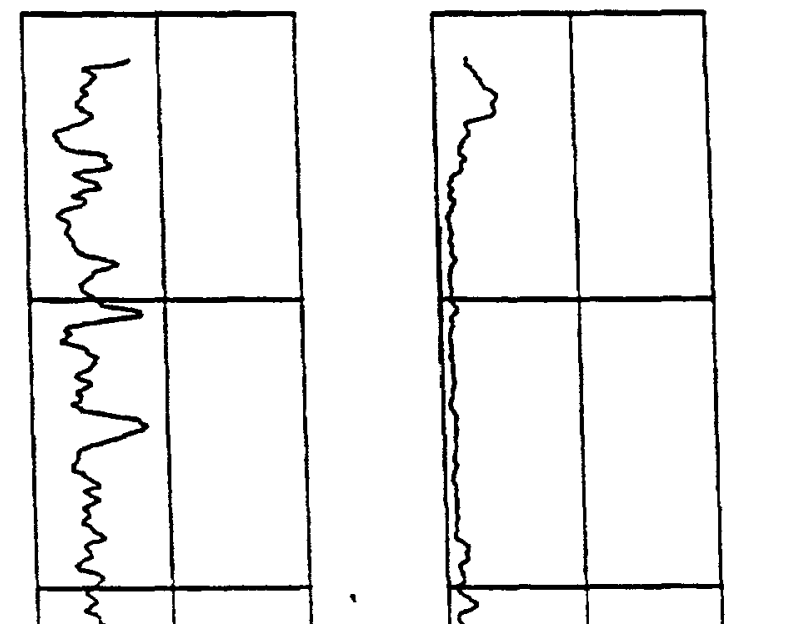


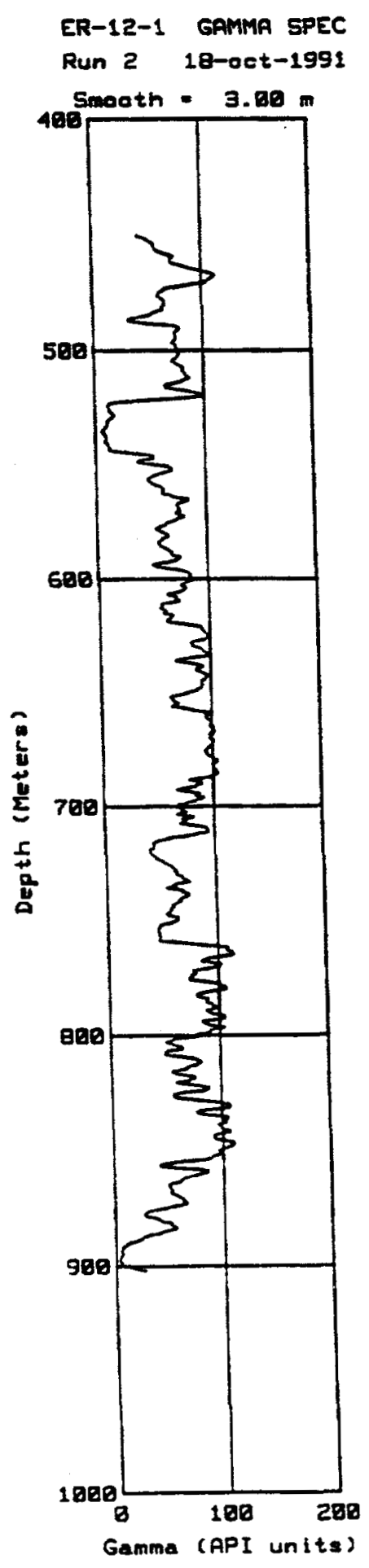

ER-12-1 GAMMA SPEC Run 2 18-oct-1991

Smooth - $3.68 \mathrm{~m}$

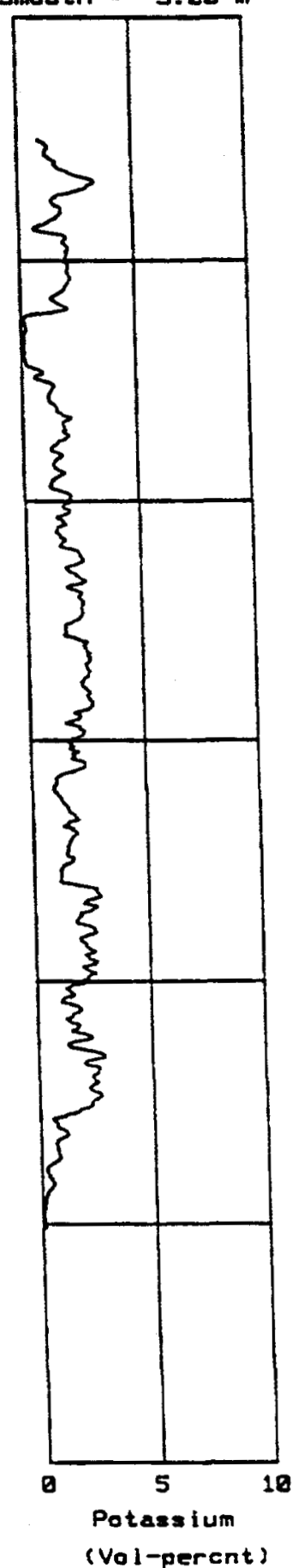

ER-12-1 GAMMA SPEC Run 2 18-oct-1991

Smoath = $3.80 \mathrm{~m}$

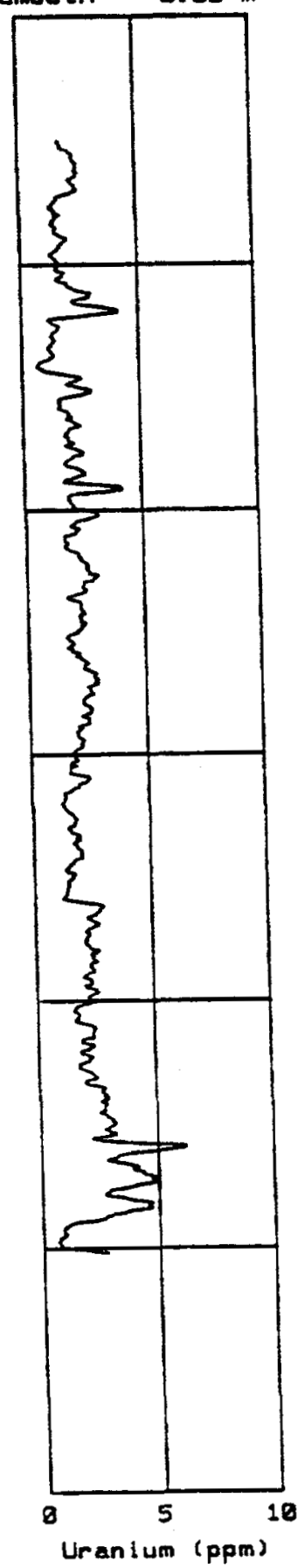

ER-12-1 GAMMA BPEC

Run 2 18-oet-1991

Smooth - $3.88 \mathrm{~m}$

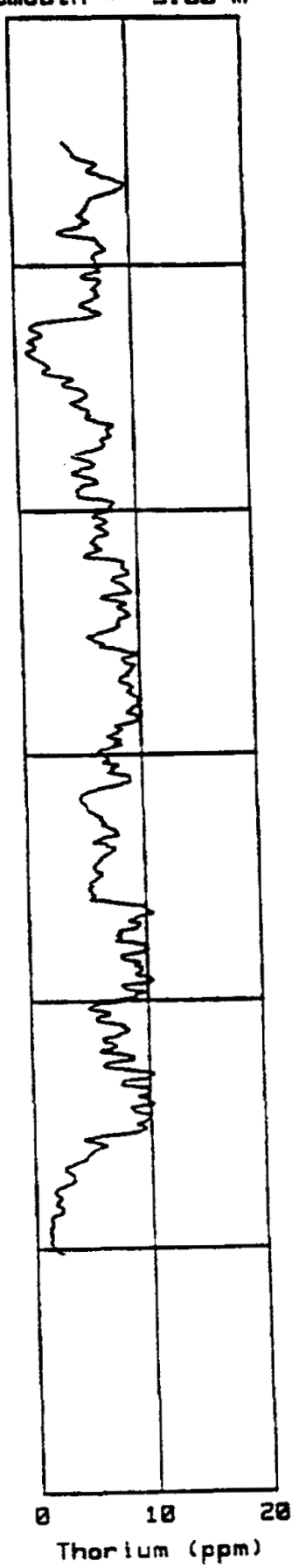

Figure 14. Spectral gamma logs, 400 to $1000 \mathrm{~m}(1312$ to $3280 \mathrm{ft})$. 

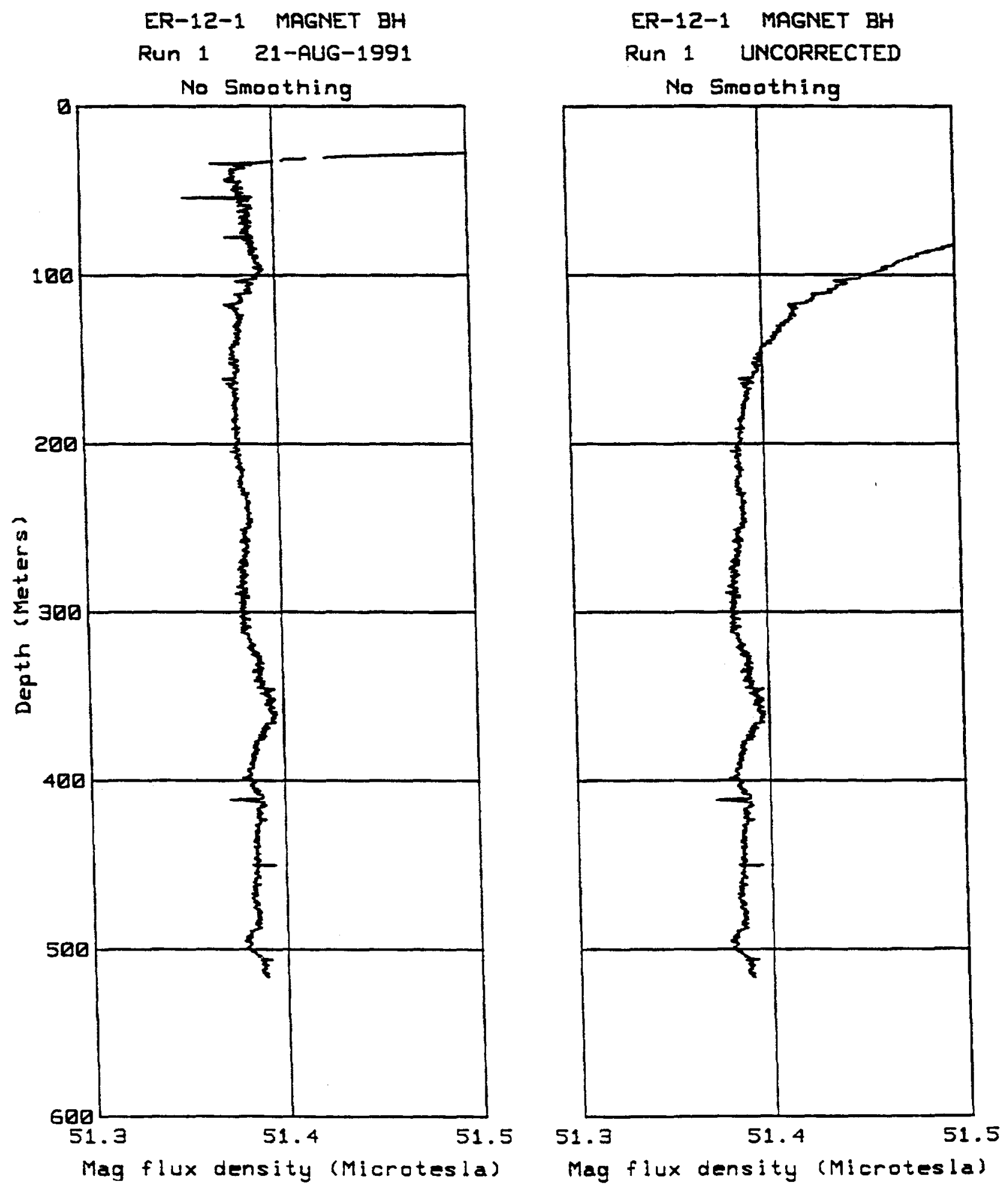

Figure 15. Borehole magnetometer logs, 0 to $600 \mathrm{~m}(0$ to $1968 \mathrm{ft}$ ). 

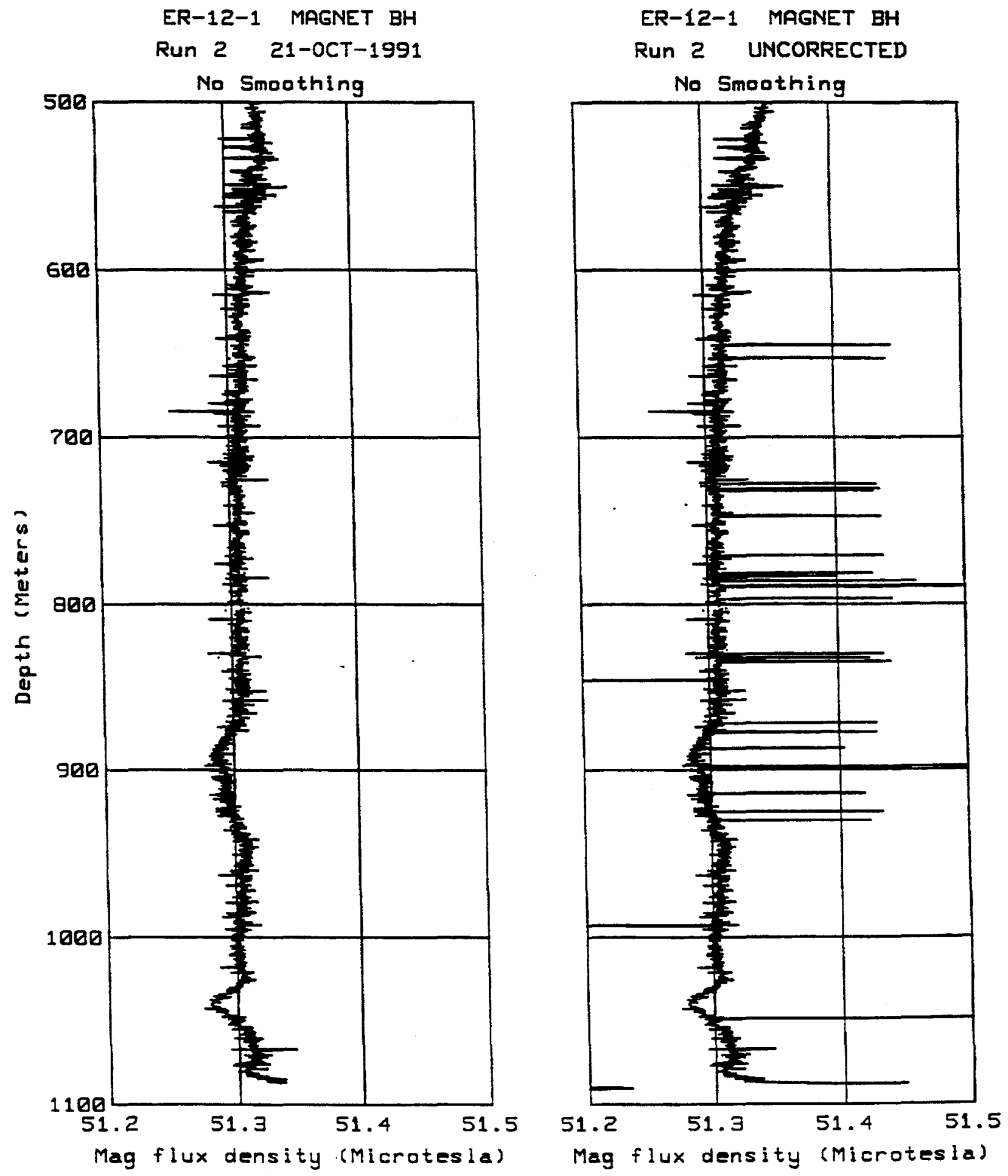

Figure 16. Borehole magnetometer logs, 500 to $1100 \mathrm{~m}$ (1640 to $3608 \mathrm{ft})$. 
The magnetic signature from the sedimentary rocks in ER-12-1 were quite subtle. When plotted on a typical scale range for NTS volcanic rock sequences, the data from Run 1 appeared as a straight line. Very little variance in the absolute values were visible. Data from Run 2 were slightly noisier. Some of the noise was due to presence of metal scraps left in the hole during the drilling process.

The accuracy of these data were on the order of 0.05 microTeslas. This level of accuracy was acceptable as these logs are typically used in a qualitative manner to recognize "signatures" of various layers. Studies have not been conducted, as far as is known, to develop characteristic signatures from magnetic logs run in sedimentary rock.

In general, it was difficult to distinguish stratigraphic contacts in hole ER-12-1 based on this $\log$ (Figures 5 and 6). A review of the data resulted in the conclusion that the magnetometer $\log$ was not an accurate indicator of the stratigraphic or lithologic units at ER-12-1. Useful information will not be gained in future boreholes, drilled into sedimentary rocks, from the total intensity magnetometer $\log$, unless they contain more magnetic material, e.g., magnetite.

\section{Sonic and Seismic Logs}

Two sonic logs were run in ER-12-1; a continuous velocity log (commonly called a CVL or $A B C)$ and a full signature sonic $\log (F R A C \log$ ) were run in the wet portion of the hole, while a

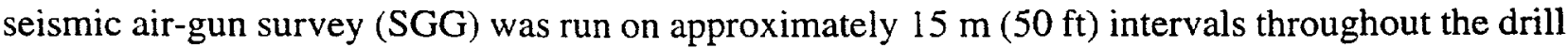
hole.

During recording of the main run of the CVL, the Atlas logging engineer determined he could improve the triggering stability by manually tracking the signal arrivals. This was done from about $899 \mathrm{~m}$ (2950 ft) to the SWL. Since the improvement was substantial, a repeat run was made with manual tracking from total depth to $899 \mathrm{~m}(2950 \mathrm{ft})$. This repeat run was combined with the upper part of the main run for data processing and archival. Processing consisted of reading the AC trace in microseconds/foot, reciprocating the data, and changing the units to $\mathrm{m} / \mathrm{sec}$. Data accuracy, as indicated by Atlas Wireline references, should be within 6 microseconds/m ( 2 microseconds/ft) for data with good signal amplitudes. The most obvious feature in the data was the difference between the high velocity Sevy Dolomite and the rest of the hole.

The FRAC log was run with the same sonde as the CVL, however, the entire signal from the two receivers were recorded over a selected time interval at each depth. LLNL instructed Atlas to record data at $4 \mu$ s intervals, collecting 1,920 samples per signal at each receiver. This resulted in adequate sampling density, preventing aliasing, and allowed for accurate arrival time picks. The $1.22 \mathrm{~m}(4 \mathrm{ft})$ and $1.83 \mathrm{~m}(6 \mathrm{ft})$ spaced receivers were recorded. Various playback options were used. To obtain arrival times of $\mathrm{p}, \mathrm{s}$, and fluid waves, and to document fractures intersecting the borehole, LLNL chose a scale of 300 to 1800 microseconds for the $1.83 \mathrm{~m}(6 \mathrm{ft})$, and 200 to 1200 microseconds

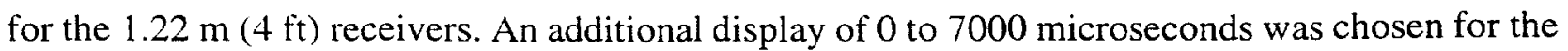
$1.22 \mathrm{~m}(4 \mathrm{ft})$ receiver to document fractures farther from the borehole. 
The first arrivals from the seismic air-gun survey appeared to be fairly good, and were modified only slightly to process the data to obtain travel time and velocity data. The accuracy of these data was $0.1 \mathrm{~ms}$.

Velocity data from the CVL and air-gun survey are presented in Figures 8 and 9 with bulk density and water content data. Travel time data from the air-gun survey are presented in Figure 17.

\section{Presentation of Data From Specific Intervals}

\section{$\underline{\text { Screened Intervals }}$}

The completion plan specified five screened and gravel-packed intervals within the carbon steel completion string for testing and sampling of the drill hole. These intervals were initially located at the following depths: 518.16 to $554.74 \mathrm{~m}$ (1700 to $1820 \mathrm{ft}$ ), 585.22 to $597.08 \mathrm{~m} \mathrm{(1920}$ to $1960 \mathrm{ft}$ ), 765.05 to $789.43 \mathrm{~m}$ ( 2510 to $2590 \mathrm{ft}$ ), 914.40 to $963.17 \mathrm{~m}$ ( 3000 to $3160 \mathrm{ft}$ ), and 1024.13 to $1048.51 \mathrm{~m}$ (3360 to $3400 \mathrm{ft}$ ). Figures $18-22$ present plots of selected log and stratigraphic data for these intervals.

\section{Cored Intervals}

Four conventional cores were cut from ER-12-1, with differing amounts of success. Core 1 was attempted through the depths of 539.19 to $543.76 \mathrm{~m}$ (1769 to $1784 \mathrm{ft})$, and recovered $0.06 \mathrm{~m}(0.2$ $\mathrm{ft}$ ) of core (1.3\%). Core 2 was attempted through the depths of 548.03 to $556.26 \mathrm{~m}$ (1798 to 1825 $\mathrm{ft}$ ), and there was no recovery. Core 3 was attempted through the depths of 751.03 to $757.73 \mathrm{~m}$ ( 2464 to $2486 \mathrm{ft}$ ), and recovered $3.0 \mathrm{~m}$ ( $10 \mathrm{ft})$ of core $(45.5 \%)$. Core 4 was attempted through the depths of 895.20 to $903.12 \mathrm{~m} \mathrm{(2937} \mathrm{to} 2963 \mathrm{ft}$ ), and recovered $7.77 \mathrm{~m}(25.5 \mathrm{ft})$ of core $(98 \%)$. Figures 23-25 present plots of selected logs and stratigraphic data for these intervals.

The scales of Figures 18-25, have been expanded in an attempt to better display the character of the data. Some data at the extreme range may have been clipped on these plots. Referring to Figures 5 and 6 , which show the entire data ranges, should provide clarifications.

\section{Lessons Learned}

Two things were learned during the conduct of logging and processing the data from ER-12-1: better ways of collecting the data, and better ways to process the data.

Data collection in the field was slower than normal as service tables were not in place for the stacked-tool runs. By the second logging run, several new service tables were developed, however, the tables included a gamma $\log$, which in reality did not speed up the logging process. This deficiency could be corrected by determining which stacked-tool configurations would be necessary to run in future drill holes, and then develop the necessary service tables (if not currently available from the oil and gas industry). The development of standard operating procedures would facilitate the use of these logs in future boreholes. 


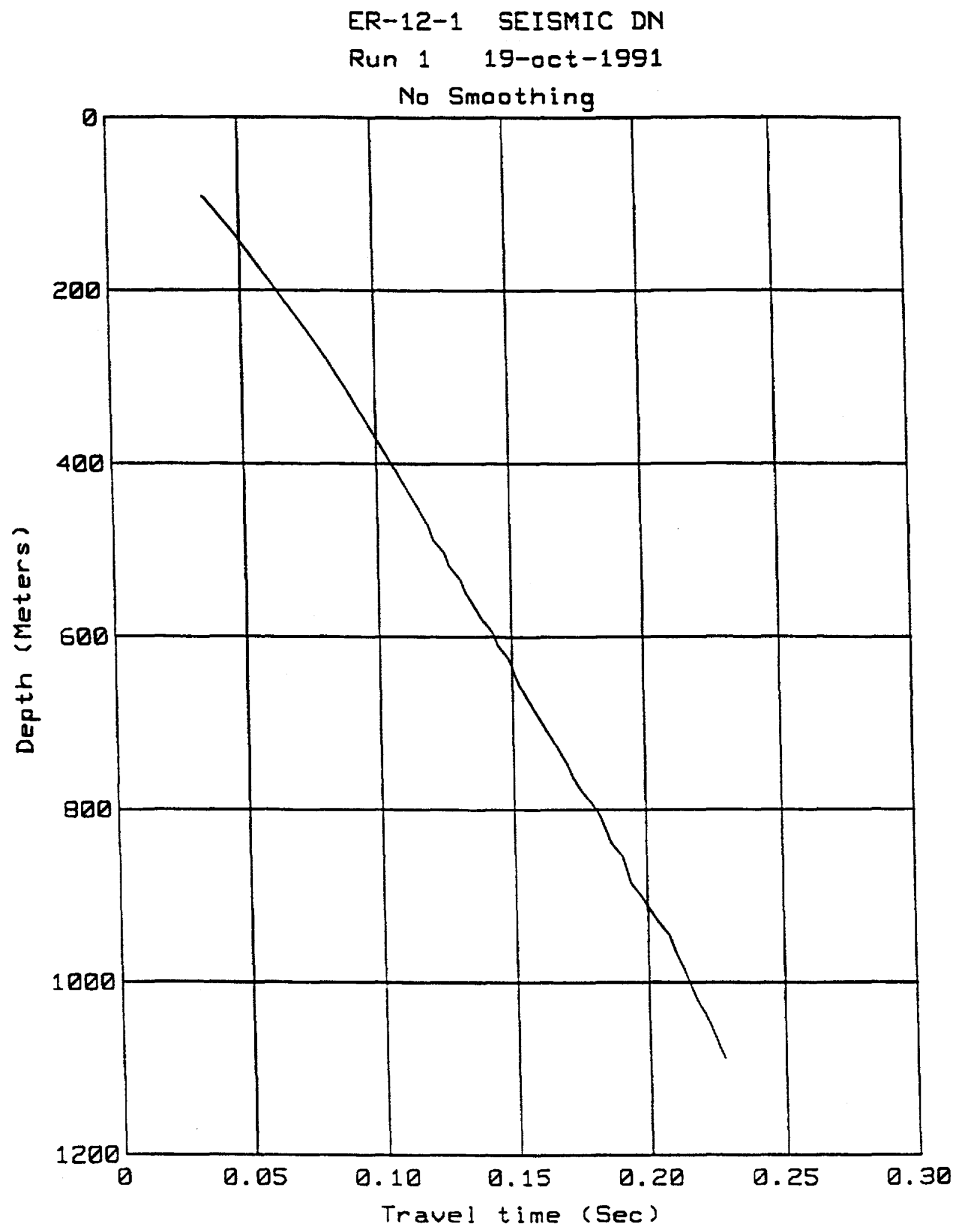

Figure 17. Travel time for the seismic air-gun survey. 


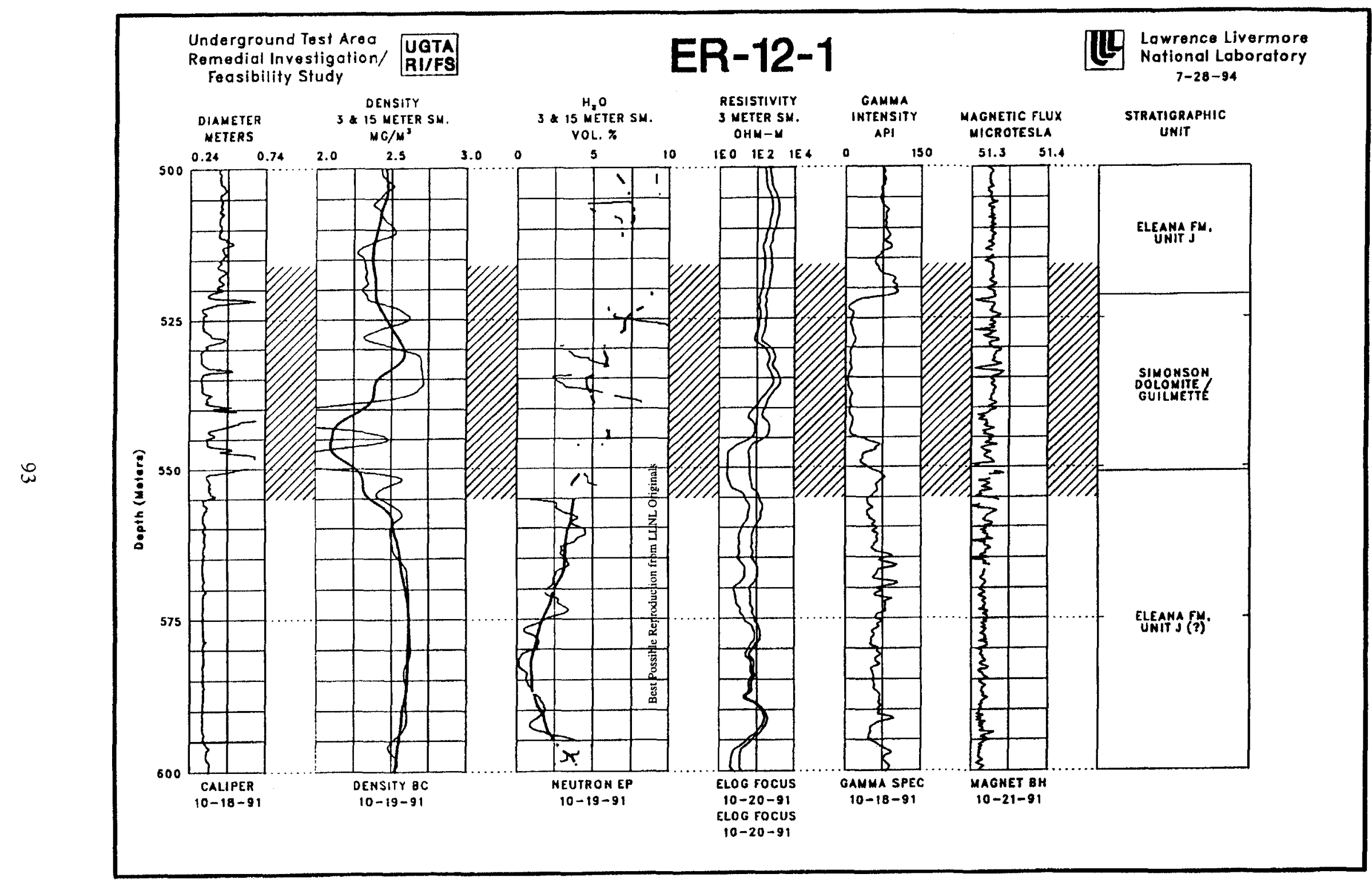

Figure 18. Selected logs for the screened interval of 518 to $554 \mathrm{~m}$ (1700 to $1820 \mathrm{ft}$ ). 


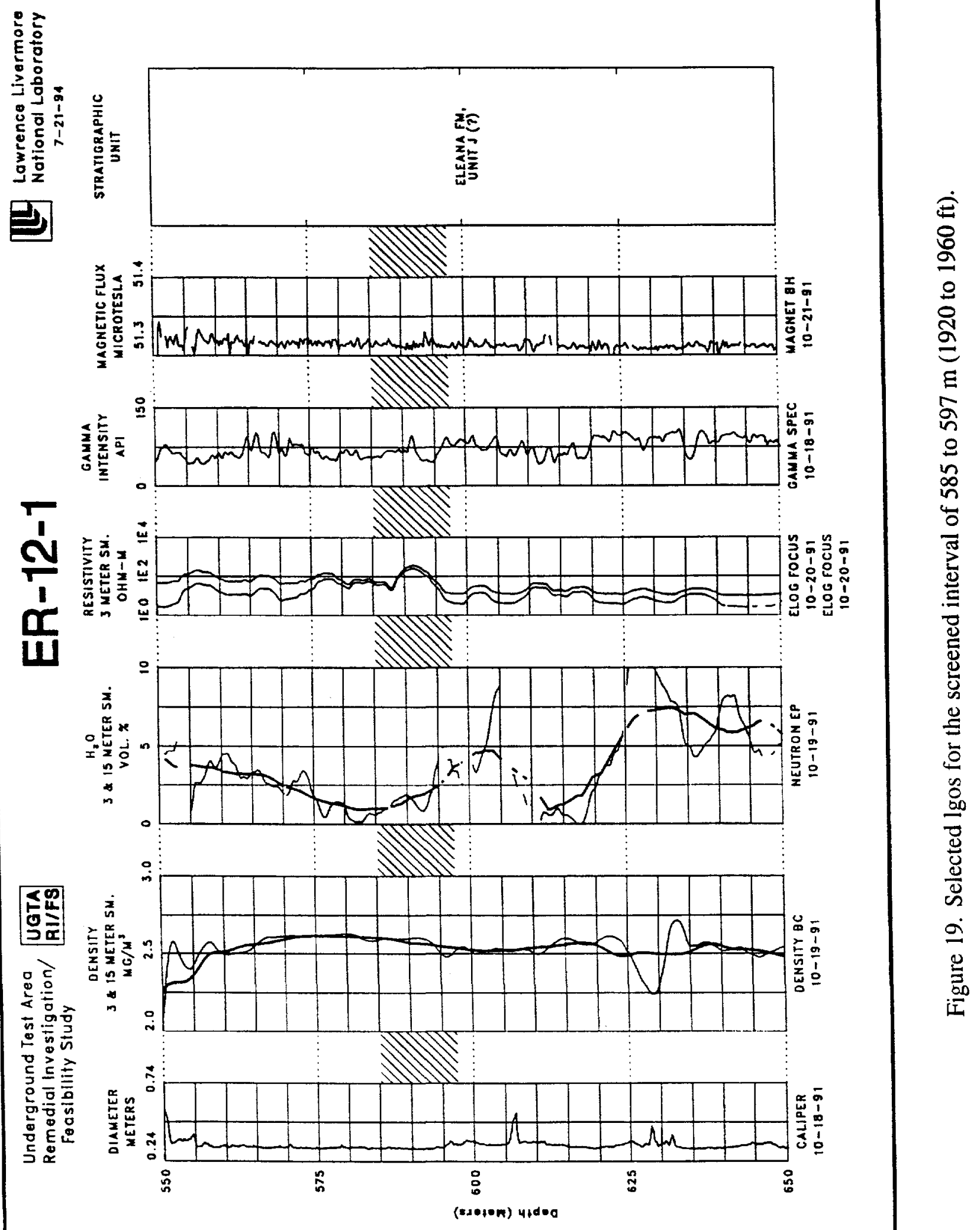




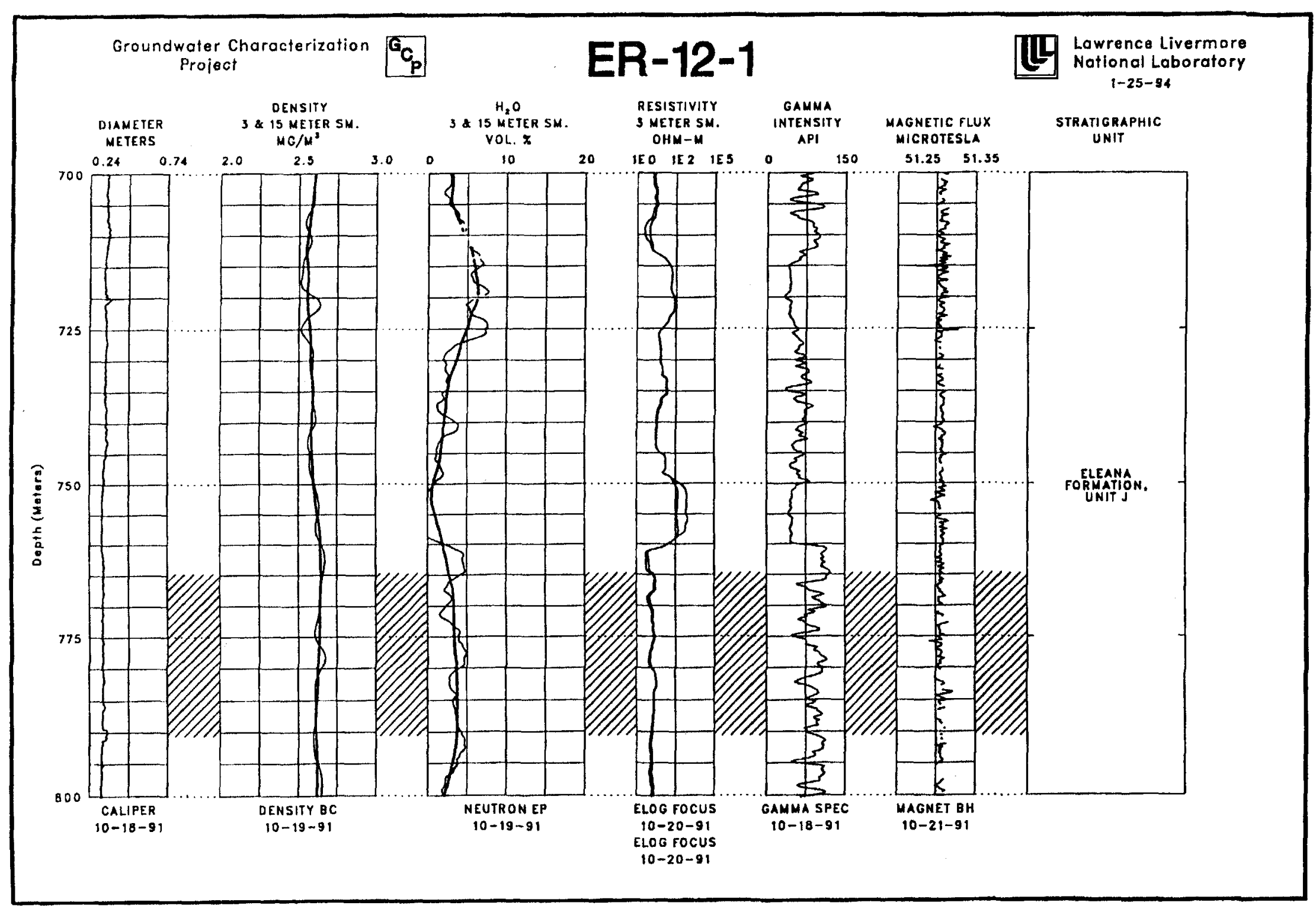

Figure 20. Selected logs for the screened interval of 765 to $789 \mathrm{~m}$ (2510 to $2590 \mathrm{ft}$ ). 

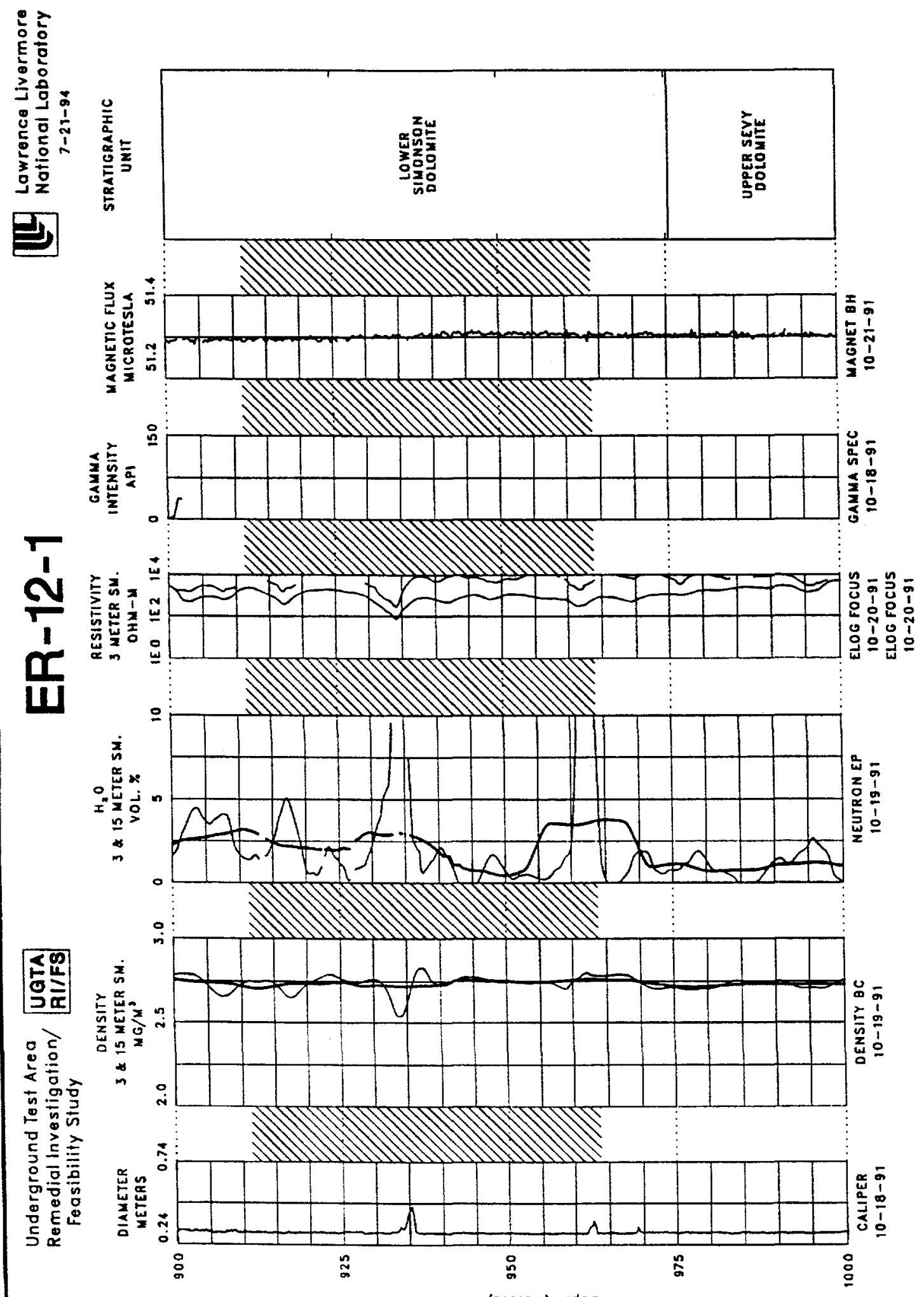

(2) 


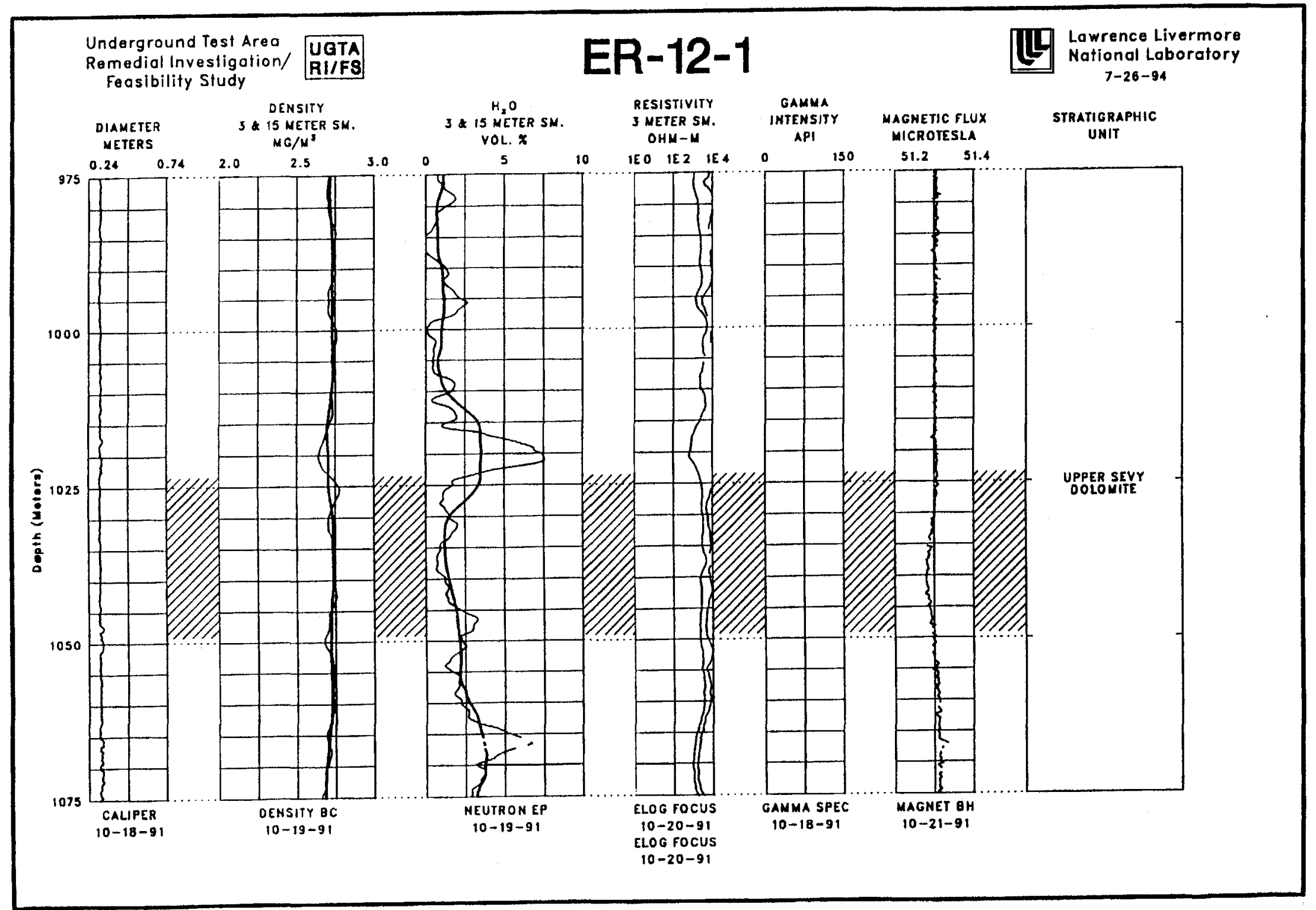

Figure 22. Selected logs for the screened interval of 1024 to $1048 \mathrm{~m}$ ( $3360 \mathrm{to} 3440 \mathrm{ft}$ ). 


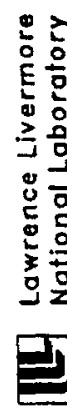
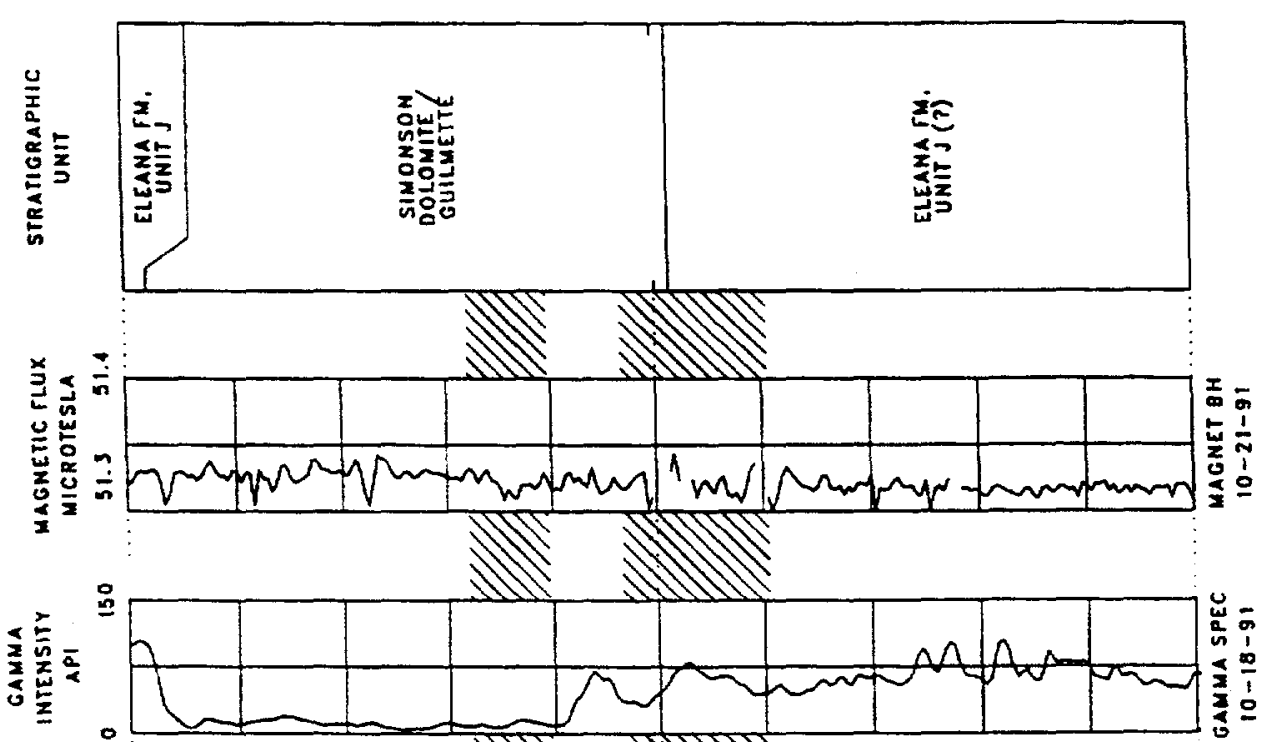

$\frac{1}{1}$
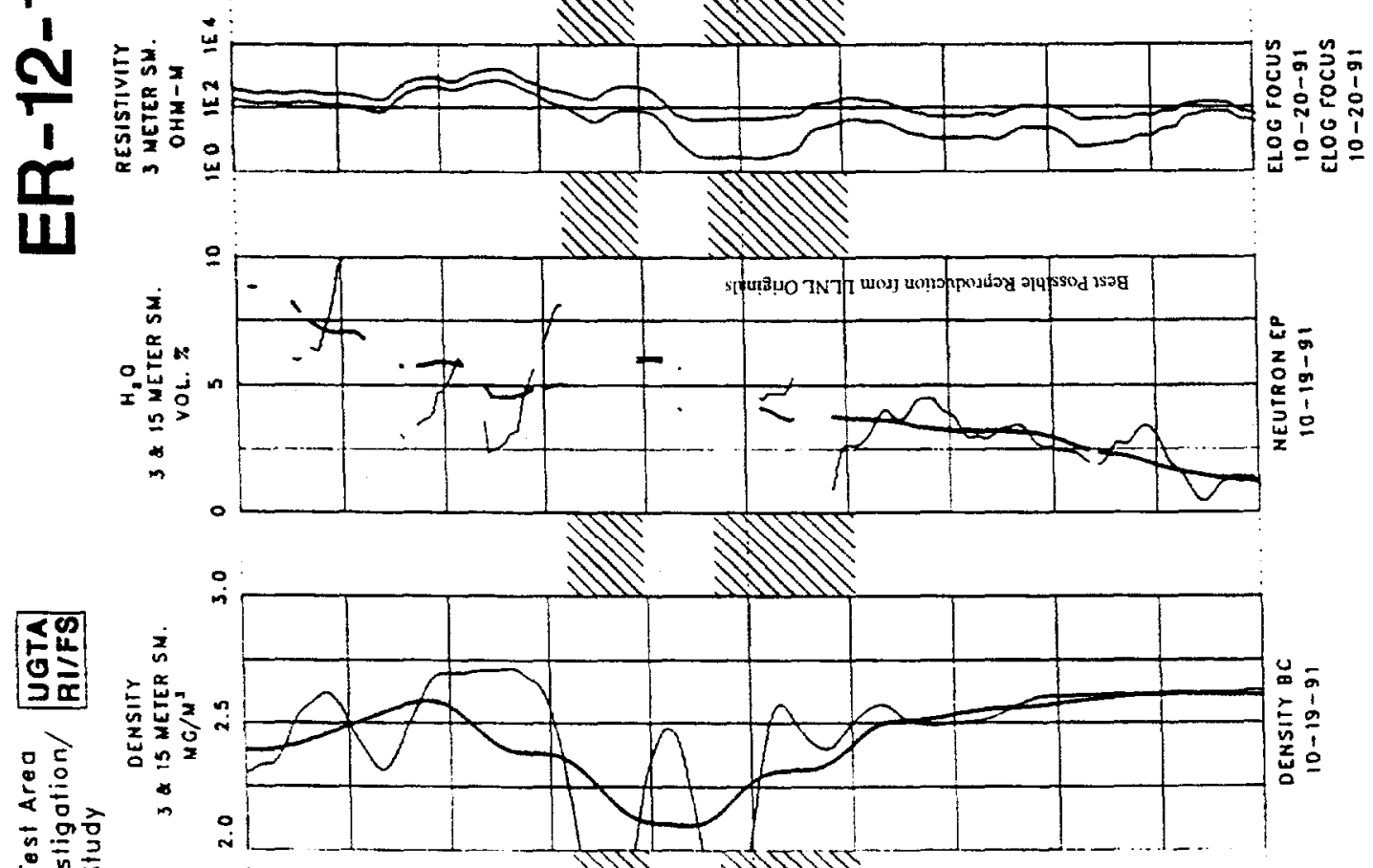

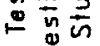

옹르를

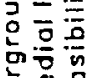

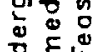

可

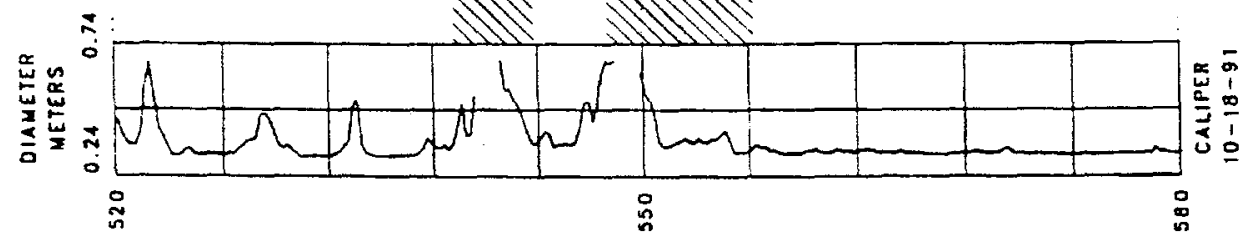

(2sectem) 41000 


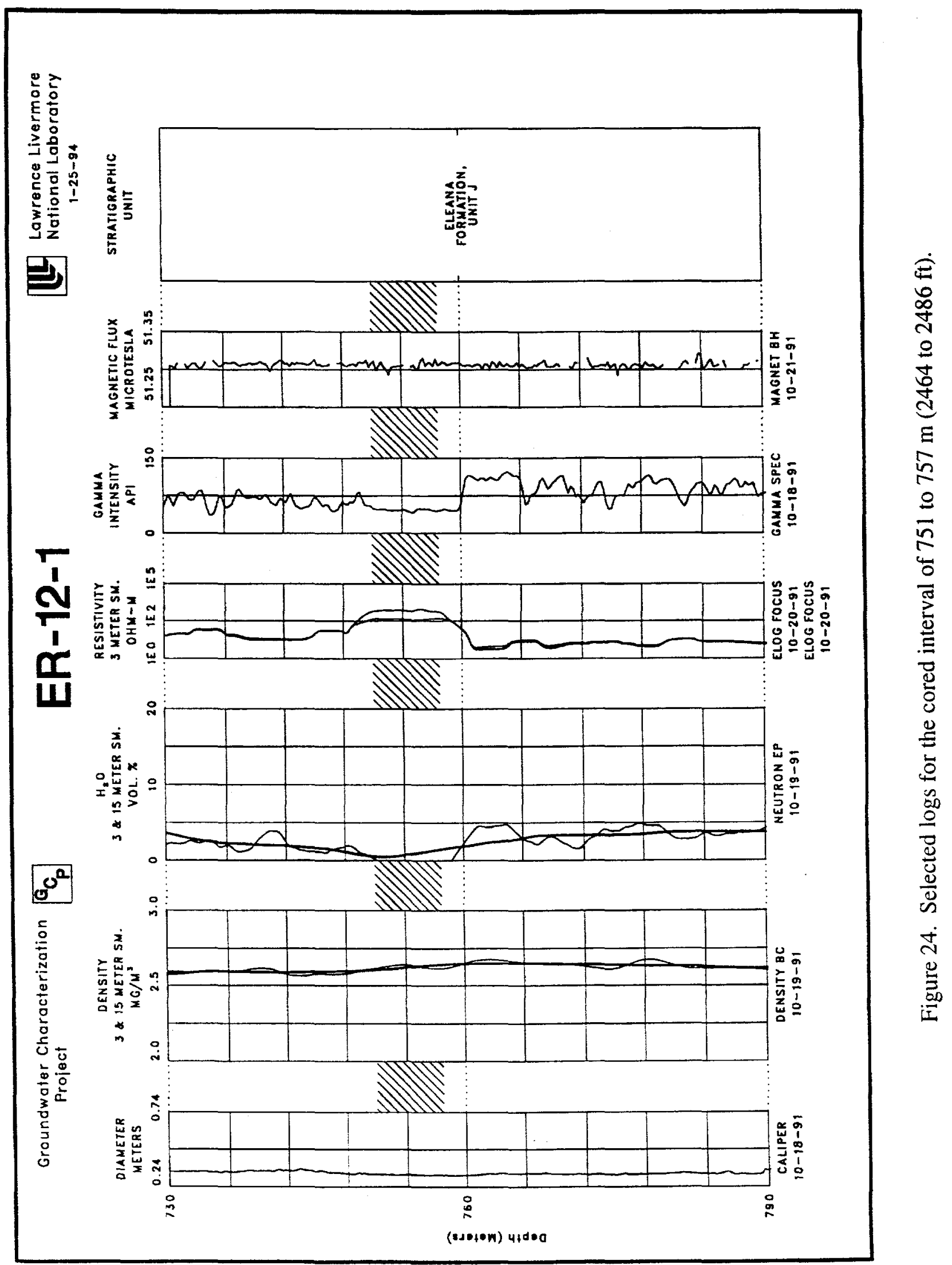




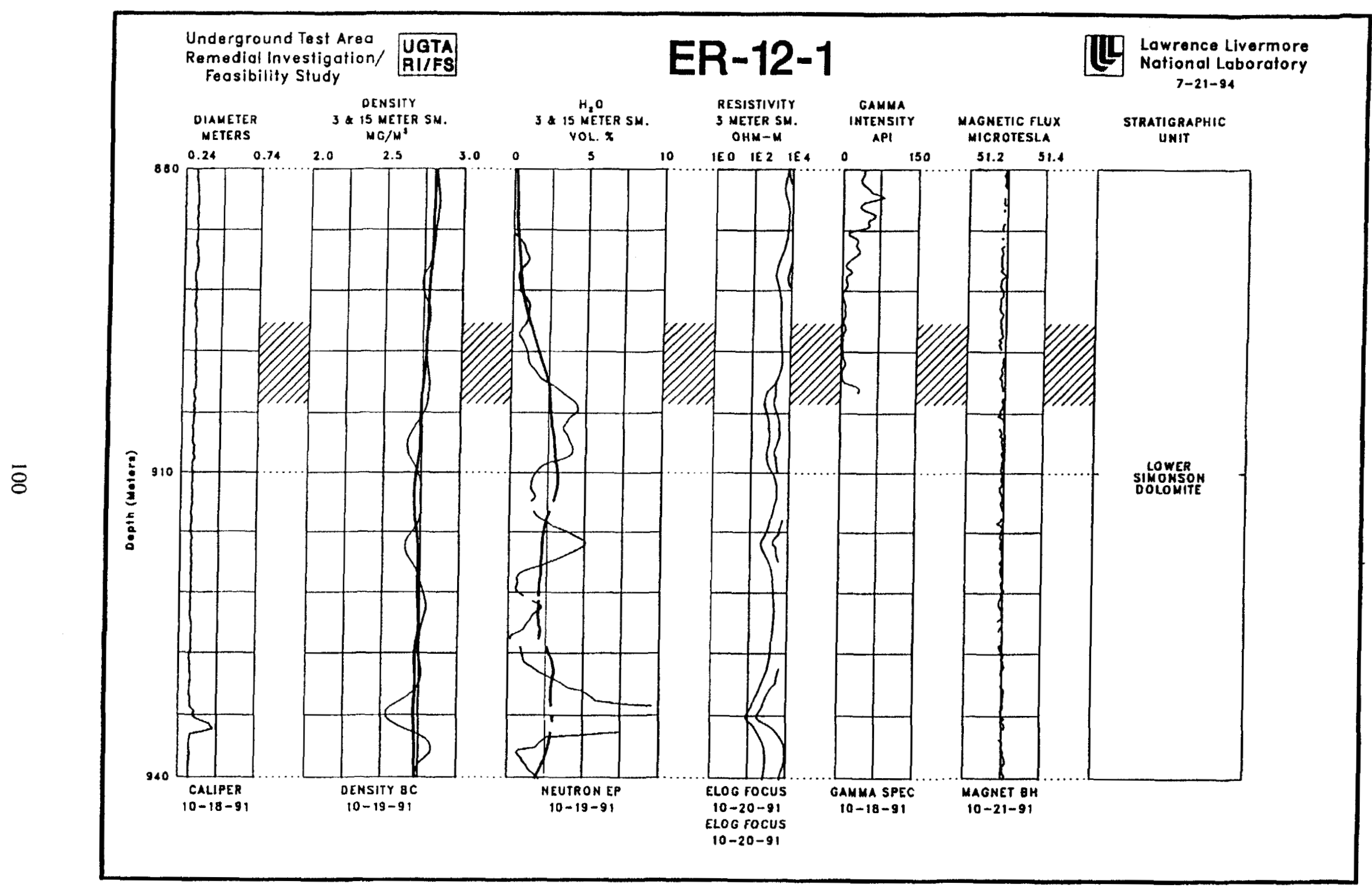

Figure 25. Selected logs for the cored interval of 895 to $903 \mathrm{~m}$ ( $2937 \mathrm{to} 2963 \mathrm{ft}$ ). 


\section{Hydrologic Testing}

\section{Water-Level Monitoring}

Static water levels at ER-12-1 were measured during drilling, development, aquifer testing, and completion (Table 33). Fluids were initially detected while drilling at a depth of $511.9 \mathrm{~m}$ (1679 ft) on August 19, 1991. Subsequent measurements indicated the static water level was approximately $470 \mathrm{~m}$ (1540 ft). This measurement represents a composite water level for all of the formations contributing water to the borehole.

Fluid levels were also determined for each of the five screened intervals during drill-stem tests conducted September 25 through October 2, 1992 (Table 34). Fluid levels measured during drill-stem test show similar hydraulic heads for the upper three screened intervals and for the lower two screened intervals. A vertical potentiometric gradient of approximately $3.3 \mathrm{~m} / \mathrm{m}$ exists through the Eleana Formation and Simonson Dolomite between the depths of $790 \mathrm{~m}(2592 \mathrm{ft})$ and $915 \mathrm{~m}(3000 \mathrm{ft})$.

\section{Flow Logs}

Two flow logs were run in ER-12-1. These logs were originally intended to be run while the borehole was uncased. Borehole instability prevented this until the hole had been cased and screened in five zones. The purpose of the logs was to identify zones within the borehole contributing

Table 33. Depth to Fluid Level and Total Depth Measurements for ER-12-1. (Modified from Drellack et al., 1991)

\begin{tabular}{|c|c|c|c|}
\hline Date & Depth to Fluid Level & Total Depth & Remarks \\
\hline $08-19-91$ & "Possible water" & $511.8 \mathrm{~m}(1679 \mathrm{ft})$ & Drilling \\
\hline $08-19-91$ & Positive indication & $520.9 \mathrm{~m}(1709 \mathrm{ft})$ & Drilling \\
\hline $08-20-91$ & $469.4 \mathrm{~m}(1540 \mathrm{ft})$ & $520.9 \mathrm{~m}(1709 \mathrm{ft})$ & USGS Iron Horse \\
\hline 09-04-91 & $472.1 \mathrm{~m}(1549 \mathrm{ft})$ & $557 \mathrm{~m}(1827 \mathrm{ft})$ & DRI bailer \\
\hline $10-18-91$ & $470.9 \mathrm{~m}(1545 \mathrm{ft})$ & $1094 \mathrm{~m}(3588 \mathrm{ft})$ & AWS DF \#4 \\
\hline $10-19-91$ & $470.3 \mathrm{~m}(1543 \mathrm{ft})$ & $1094 \mathrm{~m}(3588 \mathrm{ft})$ & AWS WNP \#2 \\
\hline $10-22-91$ & $470.9 \mathrm{~m}(1545 \mathrm{ft})$ & $1094 \mathrm{~m}(3588 \mathrm{ft})$ & $\begin{array}{l}\text { Schlumberger } \\
\text { Micro log }\end{array}$ \\
\hline $10-26-91$ & $471.2 \mathrm{~m}(1546 \mathrm{ft})$ & $1094 \mathrm{~m}(3588 \mathrm{ft})$ & CA6 \#2 \\
\hline $11-05-91$ & $468.8 \mathrm{~m}(1538 \mathrm{ft})$ & $627.7 \mathrm{~m}(1059 \mathrm{ft})$ & Static hole, hole bridged \\
\hline $11-12-91$ & $470.9 \mathrm{~m}(1545 \mathrm{ft})$ & $549.2 \mathrm{~m}(1802 \mathrm{ft})$ & CA6 \#5, hole bridged \\
\hline $04-16-92$ & $469.8 \mathrm{~m}(1541 \mathrm{ft})$ & $1094 \mathrm{~m}(3588 \mathrm{ft})$ & $\begin{array}{l}\text { Preparing for } \\
\text { thermal flow log }\end{array}$ \\
\hline 09-19-92 & $471.3 \mathrm{~m}(1546 \mathrm{ft})$ & $1044 \mathrm{~m}(3588 \mathrm{ft})$ & Developing hole \\
\hline $10-19-92$ & $468.3 \mathrm{~m}(1536 \mathrm{ft})$ & $1044 \mathrm{~m}(3588 \mathrm{ft})$ & $\begin{array}{l}\text { Preparing to install } \\
\text { sliding sleeves }\end{array}$ \\
\hline
\end{tabular}


Table 34. Static Pressures and Heads of Screened Intervals in Well ER-12-1.

\begin{tabular}{lccccc}
\hline $\begin{array}{c}\text { Screened Interval } \\
\text { Meters (ft) }\end{array}$ & $\begin{array}{c}\text { Static } \\
\text { Pressure } \\
(\mathrm{psi})\end{array}$ & $\begin{array}{c}\text { Static } \\
\text { Pressure } \\
\mathrm{m}(\mathrm{ft})\end{array}$ & $\begin{array}{c}\text { Height of } \\
\text { Pressure Gauge } \\
\text { Above Measuring } \\
\text { Point } \\
\mathrm{m}(\mathrm{ft})\end{array}$ & $\begin{array}{c}\text { Depth of } \\
\text { Pressure Gauge } \\
\text { Below Land } \\
\text { Surface } \\
\mathrm{m}(\mathrm{ft})\end{array}$ & $\begin{array}{c}\text { Static Fluid } \\
\text { Level Above } \\
\text { Mean Sea } \\
\text { Level } \\
\mathrm{m}(\mathrm{ft})\end{array}$ \\
\hline $518-555(1692-1820)$ & 131.1 & $92.1(302.0)$ & $13.2(43.4)$ & $531(1743)$ & $1331 \pm 7(4367 \pm 23)$ \\
$585-597(1920-1960)$ & 118.3 & $83.1(272.6)$ & $47.4(155.5)$ & $531(1743)$ & $1322 \pm 7(4337 \pm 23)$ \\
$765-790(2508-2592)$ & 387.1 & $271.9(891.9)$ & $47.4(155.5)$ & $706(2316)$ & $1336 \pm 7(4383 \pm 23)$ \\
$915-963(3000-3160)$ & 37.7 & $26.5(86.9)$ & $47.4(155.5)$ & $871(2957)$ & $926 \pm 7(3038 \pm 23)$ \\
$1024-1049(3360-3440)$ & 45.1 & $31.7(103.9)$ & $76.8(251.9)$ & $871(2857)$ & $931 \pm 7(3055 \pm 23)$ \\
\hline
\end{tabular}

\footnotetext{
Notes

$\pm 10 \mathrm{psi}$

$1 \mathrm{psi}=2.304 \mathrm{ft}$ of water

Static fluid level $=1540 \mathrm{ft}$ below land surface

Well-head elevation is $5819 \mathrm{ft}$
}

groundwater, and quantify the amount, from each zone. The flow logs were also used to delineate ambient circulation patterns within the borehole during equilibrium conditions. The first $\log$, Schlumberger's Oxygen Activation Flow Log, was run on April 6, 1992. This log relies upon a small neutron accelerator (minitron) to activate oxygen within the water molecule to ${ }^{16} \mathrm{~N}$, an unstable isotope of nitrogen that has a half-life of 7.10 seconds. Radioactive decay of the nitrogen isotope is monitored by gamma counters spaced along the length of the tool. The intensity of gamma radiation measured at any given counter is a function of water velocity. The results of the oxygen activation flow log are given in Table 35 and graphically presented in Figure 26. The results from the furthest detector (GR detector), agreed closely with the metered discharge at land surface. The GR detector results indicate all production from this borehole (at $1631 / \mathrm{min}$ or $43 \mathrm{gpm}$ ) emanates from the bottom $20 \mathrm{~m}(64 \mathrm{ft})$ of the 518 to $555 \mathrm{~m}$ (1700 to $1820 \mathrm{ft}$ ) screened interval. Minor fluctuations in production below this zone may indicate loss of water to under-pressurized zones, however, variance in production with depth remained within the margin of error of the technique $(0.46 \mathrm{~m} / \mathrm{min}$ or $1.5 \mathrm{ft} / \mathrm{min})$.

The thermal flow log was run on April 24, 1992. This log typically is run in conjunction with a temperature and electrical conductivity $\log$, however, on this run it was not. A thermal flow log operates by emitting a thermal pulse via a grid of heated wires in the center of the tool. The transit time of the thermal pulse up or down the borehole is monitored via fixed thermistors. The transit time is used to calculate a groundwater velocity. The thermal flow log is typically run with a packer to ensure all groundwater passes through the center of the tool. This was not the case at ER-12-1 as 
Table 35. Log Depth and Water Velocity from the Oxygen Activation Log.

\begin{tabular}{|c|c|c|c|c|}
\hline \multirow[b]{2}{*}{ Minitron Depth } & \multirow[b]{2}{*}{ Date - Time } & \multirow[b]{2}{*}{ Gamma Ray } & \multicolumn{2}{|c|}{$\begin{array}{l}\text { Velocity } \mathrm{m} / \mathrm{min}(\mathrm{ft} / \mathrm{min}) \\
\text { for each Detector }\end{array}$} \\
\hline & & & Far Detector & Near Detector \\
\hline $504.7(1655.4)$ & $4 / 6 / 92-15: 16$ & $0.0(0.0)$ & $0.9(3.1)$ & NQ (NQ) \\
\hline $511.6(1678)$ & $4 / 6 / 92-15: 18$ & $0.0(0.0)$ & $2.3(7.7)$ & $0.0(0.0)$ \\
\hline $514.6(1688)$ & $4 / 6 / 92-20: 35$ & $0.0(0.0)$ & NQ (NQ) & NQ (NQ) \\
\hline $535.4(1756)$ & $4 / 6 / 92$ & $0.0(0.0)$ & $2.5(8.2)$ & $2.6(8.9)$ \\
\hline $545.1(1788)$ & $4 / 6 / 92-20: 54$ & $6.6(21.7)$ & $5.2(17.0)$ & NQ (NQ) \\
\hline $582.3(1910)$ & $4 / 6 / 92$ & $10.8(35.3)$ & $7.7(25.4)$ & NQ (NQ) \\
\hline $591.5(1940.0)$ & $4 / 6 / 92$ & $10.5(34.5)$ & $7.7(25.4)$ & NQ (NQ) \\
\hline $759(2490)$ & $4 / 6 / 92-21: 14$ & $9.6(31.6)$ & $7.4(24.3)$ & NQ (NQ) \\
\hline $762(2500.0)$ & $4 / 6 / 92$ & $9.2(30.1)$ & $6.6(21.8)$ & NQ (NQ) \\
\hline $777(2550.0)$ & $4 / 6 / 92$ & $9.8(32.1)$ & $7.0(23.1)$ & NQ (NQ) \\
\hline $780.5(2560)$ & $4 / 6 / 92-21: 34$ & $9.4(30.8)$ & $7.0(23.1)$ & NQ (NQ) \\
\hline $908.5(2980.0)$ & $4 / 6 / 92$ & $9.7(31.7)$ & $7.8(25.8)$ & NQ (NQ) \\
\hline $929.9(3050.0)$ & $4 / 6 / 92$ & $9.8(32.0)$ & $7.8(23.6)$ & NQ (NQ) \\
\hline $948.2(3110.0)$ & $4 / 6 / 92-19: 15$ & $9.8(32.3)$ & $8.1(26.6)$ & NQ (NQ) \\
\hline 1001.8 & $4 / 6 / 92-19: 40$ & $9.9(32.6)$ & $7.8(25.7)$ & NQ (NQ) \\
\hline
\end{tabular}

Notes

Effective radius $=0.1894 \mathrm{ft}^{2}$

Pump depth $=3333.35 \mathrm{ft}$

NQ is not quantifiable

the vendor (Desert Research Institute) did not have appropriately sized packers for the $0.29 \mathrm{~m}$ (7-3/8 in) diameter borehole. Flow velocities determined by this tool should be qualitative estimates only. The results of the thermal flow $\log$ are presented in Table 36 and in Figure 27. The results indicate inflow was occurring at the bottom $12.2 \mathrm{~m}(40 \mathrm{ft}$ ) of the top screen ( 518 to $555 \mathrm{~m} ; 1700$ to $1820 \mathrm{ft}$ ) and from the third screen ( 765 to $790 \mathrm{~m} ; 2510$ to $2590 \mathrm{ft}$ ) and outflow was occurring through a 6 $\mathrm{m}(20 \mathrm{ft}$ ) section of the fourth screen between the depths of 933 and $939 \mathrm{~m}$ (3060 and $3080 \mathrm{ft}$ ).

The results of the oxygen activation flow log and the thermal flow log indicate the screened interval between $518-555 \mathrm{~m}$ ( 1700 and $1820 \mathrm{ft}$ ) has the highest production within the borehole. The thermal flow log indicates that the screened intervals between 585 to $597 \mathrm{~m} \mathrm{(1920} \mathrm{to} 1960 \mathrm{ft})$ and 765 to $790 \mathrm{~m}$ ( 2510 to $2590 \mathrm{ft}$ ) appear to have a similar pressure head as the upper screened zone, while the oxygen activation log indicates these screened intervals have relatively low production 


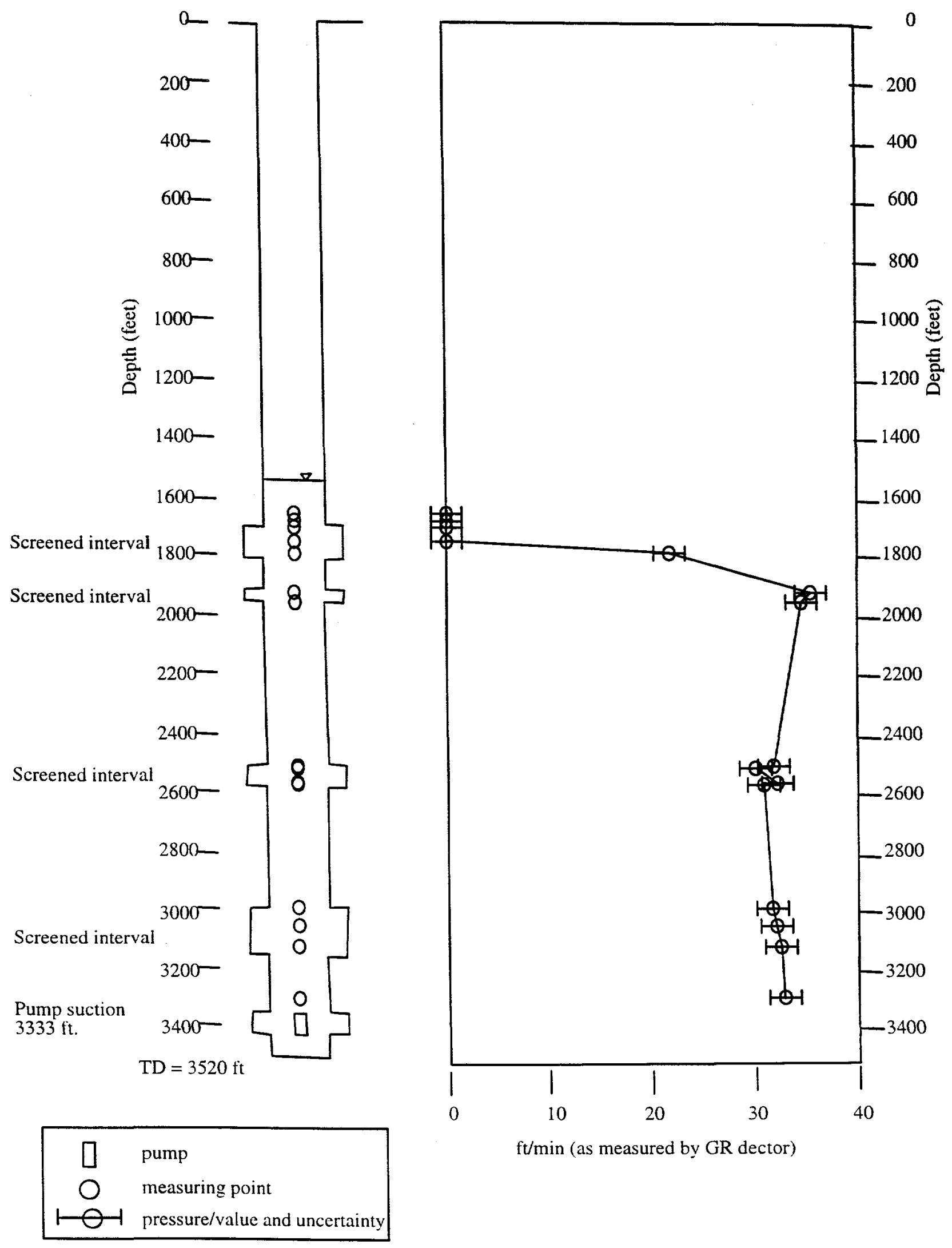

Figure 26. Results of the oxygen activation flow $\log$. 
Table 36. Ambient Flow Versus Depth, as Determined by Thermal Flow Log.

\begin{tabular}{cl}
\hline Depth m (ft) & Flow $1 / \min (\mathrm{gpm})$ \\
\hline $473(1552)$ & $<0.37(<0.1)$ \\
$516(1691)$ & $<0.37(<0.1)$ \\
$533(1750)$ & $<0.37(<0.1)$ \\
$541(1775)$ & $<0.37(<0.1)$ \\
$555(1822)$ & $-3.0(-0.8)$ \\
$582(1910)$ & $-2.27(-0.6)$ \\
$600(1970)$ & $-2.6(-0.7)$ \\
$793(2600)$ & $-4.2(-1.1)$ \\
$927(3170)$ & $-4.2(<0.1)$ \\
$933(3080)$ & $-4.2(<0.1)$ \\
$939(3040)$ & $<0.37(-1.1)$ \\
$966(3060)$ & $<0.37(-1.1)$ \\
\hline
\end{tabular}

Negative values indicate downward flow

rate. The thermal flow log also indicates markedly less formation pressure for the lower two screened intervals relative to the upper three intervals.

\section{Multiple Rate Test}

A multiple rate aquifer test was conducted at ER-12-1 on April 4, 1992. The borehole had been drilled, cased, gravel packed and screened at the following depths; 518 to $554 \mathrm{~m}$ (1700 to $1820 \mathrm{ft}$ ), 585 to $597 \mathrm{~m}$ ( 1920 to $1960 \mathrm{ft}$ ), 765 to $790 \mathrm{~m}$ ( 2510 to $2590 \mathrm{ft}$ ), 914 to $963 \mathrm{~m}$ ( 3000 to $3160 \mathrm{ft}$ ), and 1024 to $1049 \mathrm{~m}$ ( 3360 to $3440 \mathrm{ft}$ ). The borehole had been drilled using Davis Mix, bentonite mud, and polymers. Well development was attempted using sodium tetraphosphate and sodium acid pyrophosphate to remove bentonite and break down polymers. A total of 643,000 l (170,000 gal) of water were removed from ER-12-1 during well development, prior to the multiple rate test. Water was removed by air-lifting, swabbing, and pumping; details of well development are presented in Table 10.

Several attempts were made to conduct the multiple rate and step-drawdown aquifer tests on all the intervals in ER-12-1 during March 1992. The first multiple rate aquifer test was conducted using a REDA 205-hp submersible pump, at a depth of $615 \mathrm{~m}$ (2020 ft; suction) on $7.3 \mathrm{~cm}$ (2-7/8 in) O.D. 8 round tubing with $6.0 \mathrm{~cm}$ (2-3/8 in) O.D. hydril tubing strapped to the side to act as an access tube for transducers. The pump size was estimated based on drawdown and production rates during swabbing, as estimated by REECo drillers. The REDA pump was connected to a Varitech Varidrive, which is used to alter the frequency of the power being sent to the pump, controlling the pumping rate. Various pumping rates, ranging from 681 to $113 \mathrm{l} / \mathrm{min}$ ( 180 to $30 \mathrm{gpm}$ ), were tried during well 


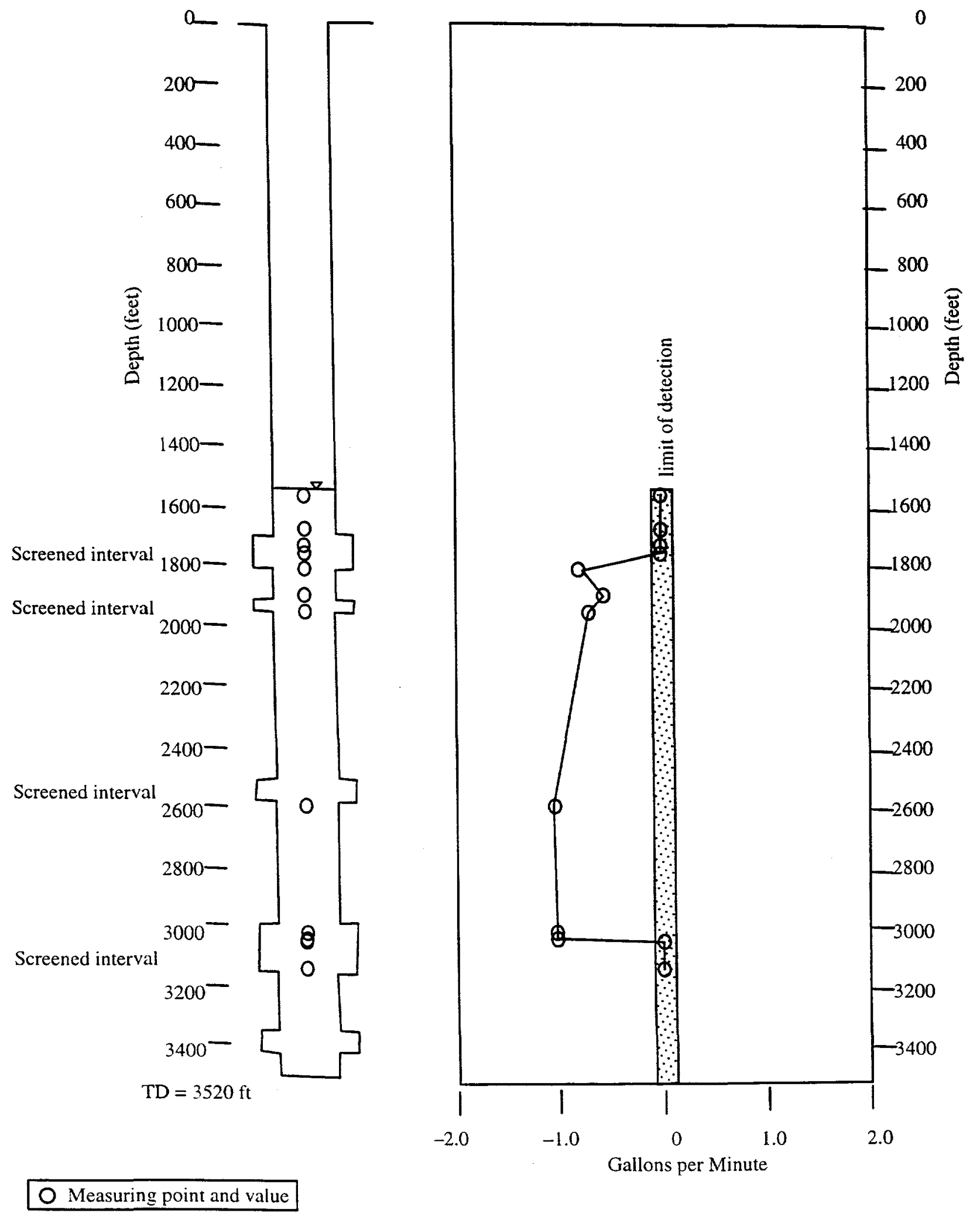

Figure 27. Results of the thermal flow log. 
development, testing the electrical system, and initial attempts to start the multiple rate aquifer test. These pumping rates were determined to exceed the production capacity of the well. Development was discontinued on March 6, 1992, until a smaller pump could be installed. A REDA 75-hp pump was installed at a depth of $764 \mathrm{~m}$ (2505 ft; suction) on March 12, 1992. The pump was run on 7.3 $\mathrm{cm}(2-7 / 8 \mathrm{in})$ O.D. 8 round tubing with $6.0 \mathrm{~cm}$ (2-3/8 in) O.D. hydril tubing attached to the side as a monitoring line. Development continued at approximately $1001 / \mathrm{min}$ (27 gpm) until March 16, 1992 , when a 500 psi geokon transducer and wireline were installed in the monitoring tube to a depth of $653 \mathrm{~m}(2144 \mathrm{ft})$.

Several problems were encountered with various electronic systems during the operation of the REDA 75-hp pump. The scintillation counter used for tritium monitoring and the geokon transducer were severely affected by signal noise. Tritium analyses and downhole pressure readings were impossible to obtain during operation of the pump, even though the two systems were on entirely separate power supplies. The first multiple rate aquifer test was conducted on March 16, 1992. Transducer signal noise problems caused the test to be aborted. The following two weeks were spent trouble-shooting the cause of the electrical problems. The varidrive was identified as a potential reason for noise in the signal, with proximity of the power cable to the transducer wireline and harmonics within the power supply creating sympathetic voltage fluctuations in the wireline. It was also thought that the wireline may have had a short in it. Atlas Wireline Service was called in and field equipment was modified to use their wireline to conduct the aquifer tests. Resultant pressure data appeared stable, however, temperature data appeared to continue to fluctuate. Examination of the pump power cable, following the multiple rate and long-term aquifer test on April 12,1992, revealed that it had been sliced open, most likely during installation, and the power cables exposed. The exposed cable was the likeliest reason for signal noise problems during the aquifer tests.

The multiple rate test was conducted to determine the optimal rate for the long-term aquifer test. The multiple rate test was conducted on April 4, 1992. Seven pumping rates, averaging 87, 121, $144,166,193,220$ and $242 \mathrm{l} / \mathrm{min}(23,32,38,44,51,58$, and $64 \mathrm{gpm})$ were used, with the duration of each step being 30 minutes long. The test was aborted at 1400 hrs on April 4, 1992, due to excessive drawdown. Recovery was monitored from $1400 \mathrm{hrs}$ until $1730 \mathrm{hrs}$, at which time the long-term aquifer test was initiated. Logistical constraints prevented a longer recovery period. The downhole pressure data obtained during the multiple rate and long term aquifer tests are given in Figure 28. Total drawdown during the multiple rate test was $78.8 \mathrm{~m}(258.6 \mathrm{ft})$ Barometric efficiencies were not calculated due to noise in the downhole pressure signal large enough to mask perturbations due to barometric changes. Barometric pressures recorded during the multiple rate and step drawdown test are presented in Figure 29. Barometric changes during the test were $0.09 \mathrm{~m}(0.3$ $\mathrm{ft}$ ) or less.

Data collected during the multiple rate aquifer test included time (fractional Julian days), downhole and atmospheric pressure (psi and feet of head), rate of discharge (gpm) and downhole temperature $\left({ }^{\circ} \mathrm{C}\right.$ ). Data were collected and stored in Campbell CR10 dataloggers and periodically backed up on the hard drive of a field computer. Data conversions in the field include converting 


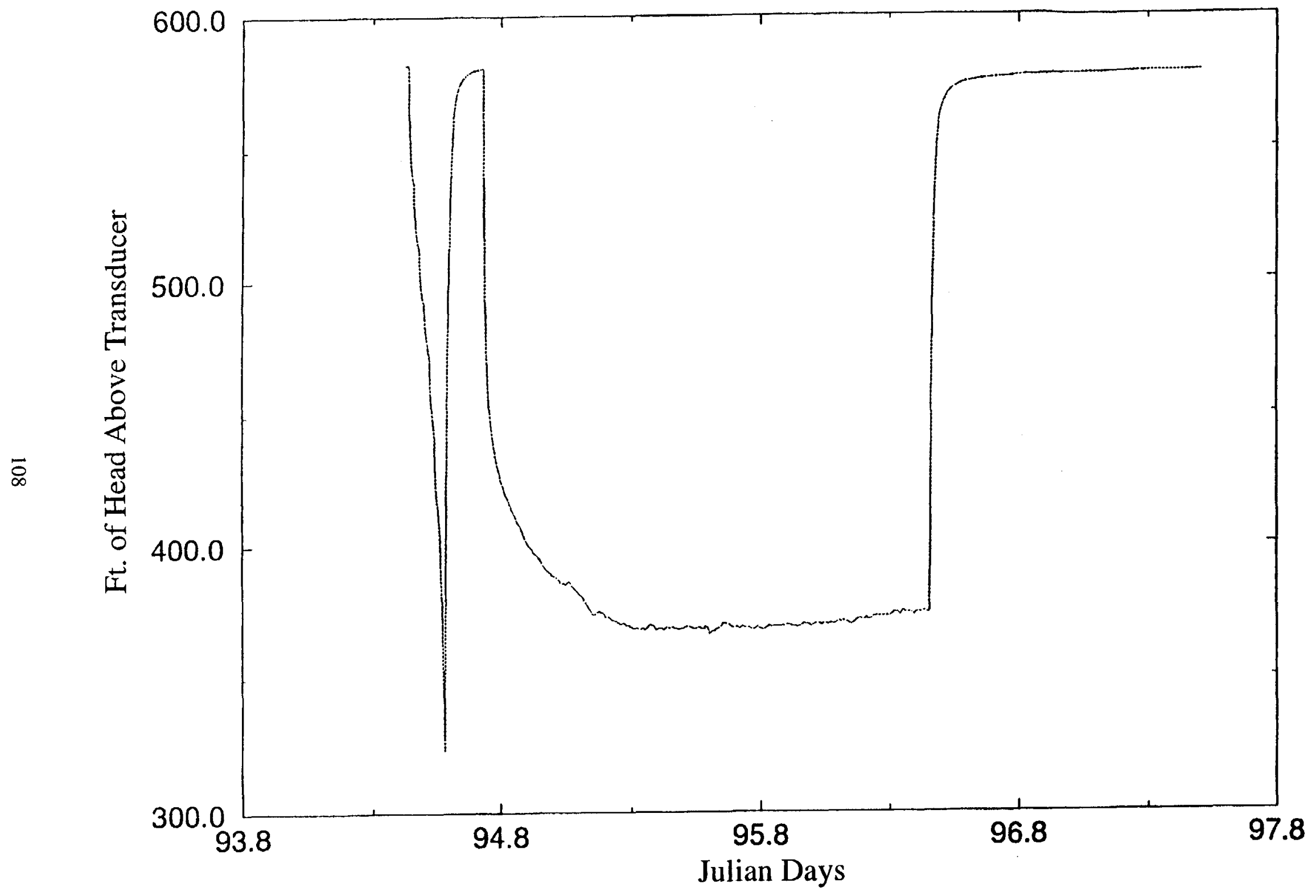

Figure 28. Downhole pressure response during multiple-rate and long-term aquifer test. 


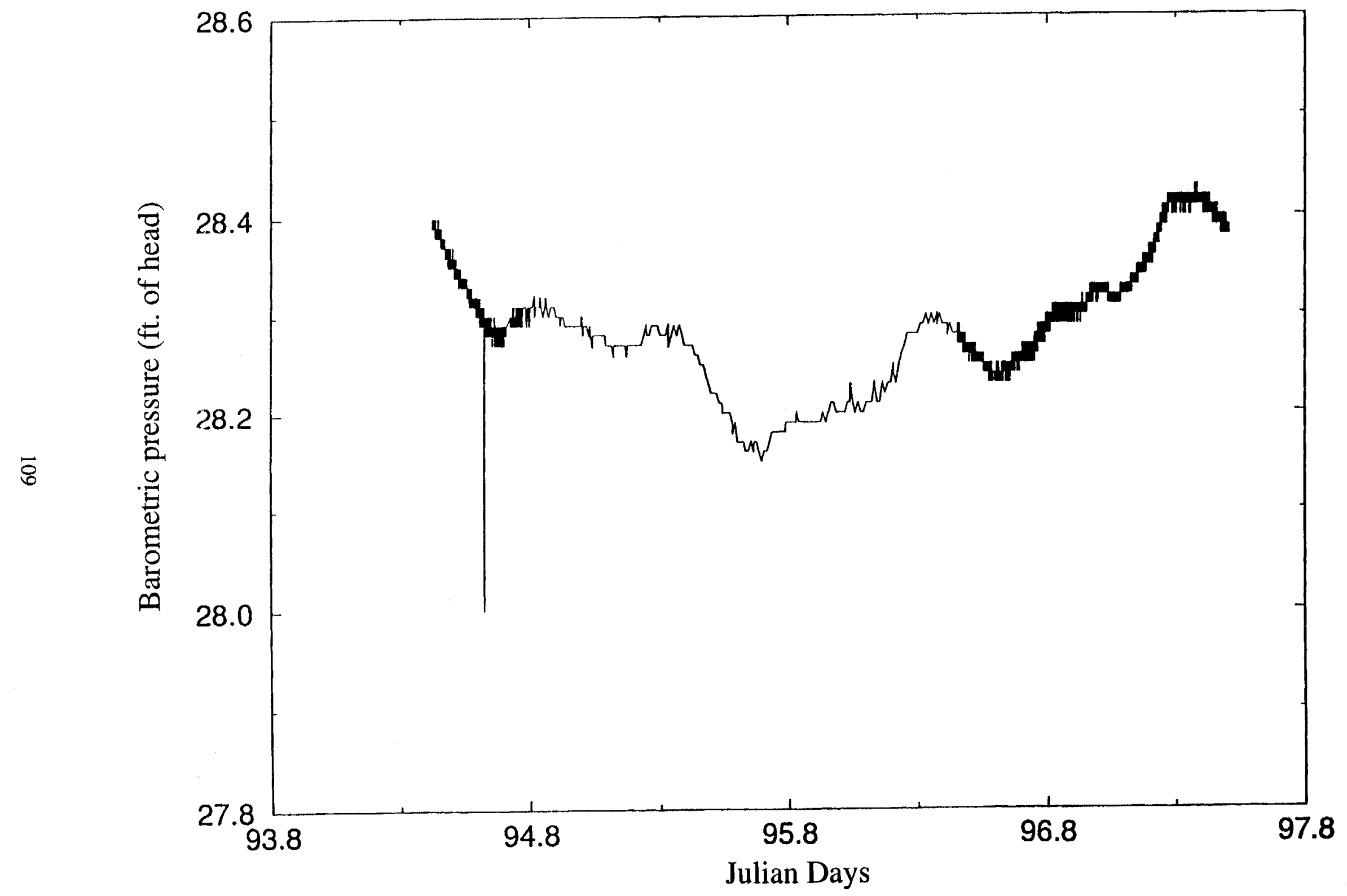

Figure 29. Barometric pressure changes during multiple-rate and the long-term aquifer test. 
frequency data collected from vibrating wire transducers into temperature corrected psi (Equation 1) and feet of head (Equation 2) and mv data for temperature into ${ }^{\circ} \mathrm{C}$ (Equation 3 ).

$$
\mathrm{P}_{(\mathrm{PSI})}\left(\left(\mathrm{M} \cdot \mathrm{kH}_{2}{ }^{2}\right)+0\right) \cdot \mathrm{T} \cdot \mathrm{C}
$$

where

$\mathrm{P}_{(\mathrm{PSI})}=$ pressure in PSI - temperature corrected

$\mathrm{M}=$ multiplier supplied with transducer from factory

$\mathrm{O} \quad=$ offset supplied with transducer from factory

$\mathrm{kH}_{2}=$ frequency response of downhole vibrating wire transducer

$\mathrm{T}=$ downhole temperature in ${ }^{\circ} \mathrm{C}$

$\mathrm{C} \quad=$ factory supplied temperature calibration factor

$$
\mathrm{P}_{(\mathrm{ft})}=\mathrm{P}_{(\mathrm{PSI})} \cdot 2.308
$$

where

$\mathrm{P}_{(\mathrm{ft})} \quad=$ pressure in feet - temperature corrected

$$
T=43.089 m_{v}^{5}-240.91 m_{v}^{4}+544.27 m_{v}^{3}-611.59 m_{v}^{2}+378.11 m_{v}-104.78
$$

where

$\mathrm{T} \quad=$ temperature in ${ }^{\circ} \mathrm{C}$

$\mathrm{m}_{\mathrm{y}} \quad=$ millivolt reading from downhole

polynomial is empirically derived and used for all thermistors

The multiple rate aquifer test suggested that a pumping rate of approximately $151 \mathrm{lpm}(40 \mathrm{gpm})$ would be sustainable for long periods of time at ER-12-1.

\section{Radially Converging Aquifer Test}

Well ER-12-1 was allowed to equilibrate for a period of 3.5 hours following the end of the multiple rate aquifer test. The long-term radially converging aquifer test was initiated at $1730 \mathrm{hrs}$ on April 4, 1992, at a rate of $155 \mathrm{l} / \mathrm{min}(41 \mathrm{gpm})$ and was continued until $1100 \mathrm{hrs}$. on April 5, 1992. Recovery was monitored until 1200 hrs on April 6, 1992.

The data collected during the aquifer test are presented in Figure 30. The data were analyzed using interpretive software entitled Well Hydraulics Interpretation Program (WHIP) version 3.22 


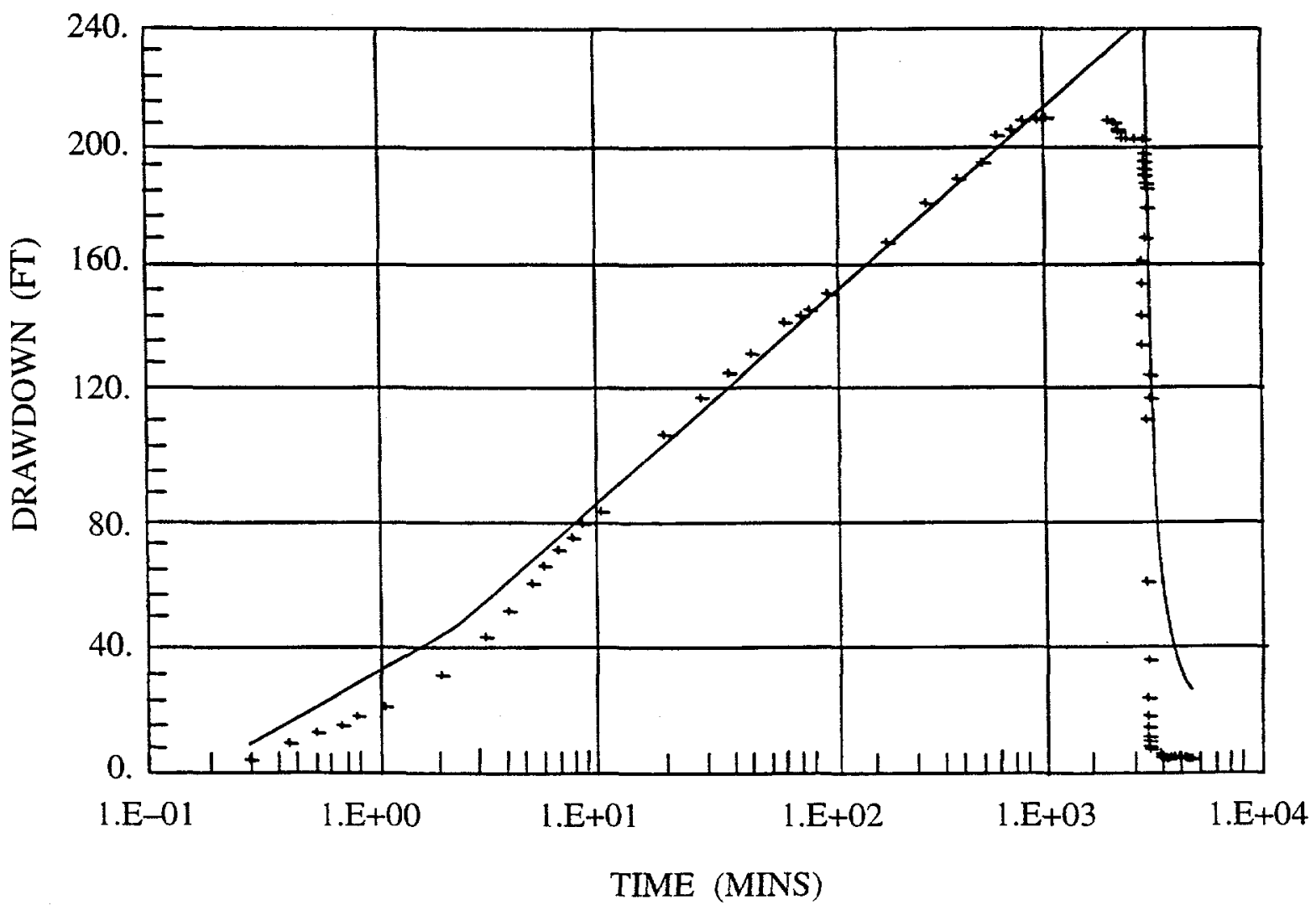

Figure 30. Cooper-Jacob analysis of drawdown data from long-term aquifer test at ER-12-1, April 4, 1992.

by Hydro GeoChem Inc., 1988. The initial analysis entailed plotting the drawdown and recovery data in terms of drawdown below initial fluid level versus the log of total elapsed time and plotting the best fitting straight line through the data according to Cooper and Jacob (1946) while accounting for deviations in early drawdown due to borehole storage. The best fitting line was determined by least squares best fit through the data points obtained during the period of 10 to 800 minutes following the start of the test. The data collected during this time period appeared to be characterized by a straight line with positive slope, indicating pseudo-steady-state radial flow (Kruseman and de Ridder, 1992). The transmissivity and storativity determined by this method were also used to calculate a type curve for the recovery data (Theis, 1935). A comparison of the measured drawdown and recovery to the theoretical drawdown indicates that the early drawdown data from the aquifer test was affected by well bore storage. The latter drawdown data as well as all of the recovery data were affected by either continuing well development or the presence of a zone within the well, with 
a lower head, that began to contribute additional groundwater only after head within the well declined to a certain point. The Cooper-Jacob (1946) analysis resulted in an estimated transmissivity of $2.4 \times 10^{-5} \mathrm{~m}^{2} / \mathrm{s}$ and a storativity of 0.15 . Storativity estimates derived from drawdown data taken from the pumping well are subject to several types of errors including uncertainties in effective radius of the well. It is likely that this storativity estimate is in error by several orders of magnitude. Alternatively, Moench (1984) reported a storativity value of 0.15 derived from drawdown data taken from UE-25b\#1, a well completed in fractured Tertiary volcanics. Moench (1984) attributed the large storativity value to the presence of highly compressible microfissures in the rock matrix. These conditions may exist at ER-12-1 (Table 28).

Theoretical drawdowns were generated using analytical solutions in the laplace transform space to the differential equations representing the response of groundwater flow systems to hydraulic stress tests (Hydro GeoChem, 1988). The solutions are of the general form

$$
H(d, z, p)=Q(z) * I(d, z, p)
$$

where:

$\mathrm{H}()=$ laplace transform of the drawdown

QO = laplace transform of the pumping history

$\mathrm{I}()=$ laplace transform of the aquifer impulse response function

$\mathrm{d}=$ dimensionless distance from the center of the pumped well

$\mathrm{p} \quad=$ hydraulic parameters of the system

$\mathrm{z} \quad=$ laplace transform variable corresponding to dimensionless time.

The impulse response function is based upon Lai and $\mathrm{Su}$ (1974). The pumping rate function is based upon a solution developed by Gupta (1985). The drawdowns in the real domain are obtained by numerical laplace inversion using Stehfest (1970).

The analytical solutions were used to generate several theoretical drawdowns that matched the observed data set. Input variables were iteratively altered with the purpose of estimating key hydrologic parameters. The results are presented in Figure 31. Theoretical drawdowns that utilized a transmissivity of $2.4 \times 10^{-5} \mathrm{~m}^{2} / \mathrm{s}$ and a storativity of 0.11 appeared to create the best fit to the observed data. The theoretical effective radius is smaller than the effective radius $(0.229)$ calculated from the casing minus the pump column, transducer column, and power cable. The discrepency may be due to fluid loss from the upper three zones to the lower three. The fluid loss would decrease effective borehole storage.

The transmissivity and storativity values estimated by this technique are very close to those determined by the Cooper and Jacob (1946) method. Poor fits to the early and late portions of the drawdown curve and the late portion of the recovery curve remained. The early variation of the drawdown curve from the analytical solution may have been due to variations in pumping. Variance 


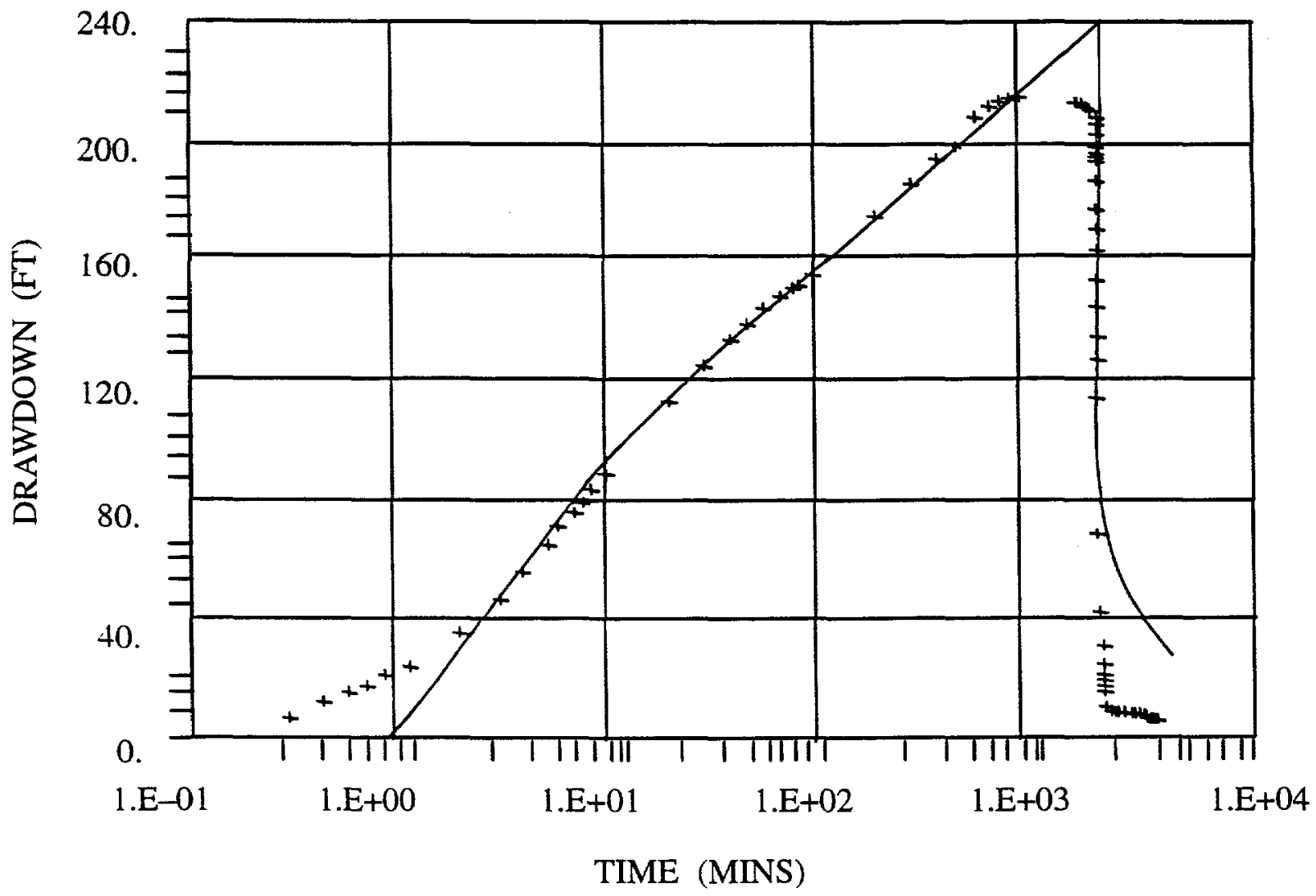

Figure 31. Hydrologic parameter estimation of ER-12-1 aquifer test, April 4, 1992.

from the analytical curve during the latter portion of drawdown may have been due to well development occurring during pumping or to the development of one of the lower zones that are under-pressured (see results of drill-stem tests). Development of one of the lower pressure zones would only occur when fluid pressures within the pumped borehole dropped below the static pressure in the under-pressured zones, stopping potential fluid loss into that zone and allowing fluid production to begin. This process would create a situation where the rate of fluid decline would slow or even possibly reverse. This process would affect recovery by causing a more rapid recovery than expected until pressure in the borehole exceeded that of the under-pressured formation, at which time the rate of recovery would be reduced. The data contained in Figures 30 and 31 appear to fit the proposed explanation, however, this does not rule out other possibilities.

\section{Drill-Stem Tests}

Drill-stem tests were conducted on three of the five screened intervals during the period of September 25 through October 2, 1992. These tests were conducted using a Schlumberger drill-stem test tool configured with thermistors and 0 to 15,000 psi pressure transducers above, below, and 
within the packer interval and an MSRT 104 pressure gauge with real time data collection on the surface and downhole.

The transducers have an accuracy of $10 \mathrm{psi}$ and a repeatability of $0.1 \mathrm{psi}$. Three drill-stem tests were conducted: the first was conducted on the screened interval of 915 to $963 \mathrm{~m}$ (3000 to $3160 \mathrm{ft}$; lower Simonson Dolomite), the second from 765 to $790 \mathrm{~m}$ (2510 to $2590 \mathrm{ft}$; Upper Eleana Formation), and the third from 585 to $597 \mathrm{~m}$ (1920 to $1960 \mathrm{ft}$; Upper Eleana Formation). The pressure response for each of the three tests are presented in Figures 32 to 34 . The first drill-stem test was an injection test where fluid was introduced into the borehole. The two other drill-stem tests were withdrawal tests where fluid was withdrawn from the borehole into an evacuated space within the drill-stem test tool and drill-pipe. Formation pressures were allowed to approach equilibrium prior to starting each drill-stem test. Ambient formation pressures of each of the three screened intervals are given in Table 34. These pressures indicate the two lowermost zones are under-pressured by approximately $396 \mathrm{~m}(1300 \mathrm{ft})$, relative to the three uppermost zones; a conclusion in complete agreement with the results of the thermal flow logs and the interpretation of the composite long-term radially converging aquifer test.

Pressures were monitored above and below the packed-off interval during the drill-stem tests. The purpose for collecting these data was to determine if the packers, well annulus, and geologic formations were allowing a detectable quantity of fluid to vertically migrate, from one screened interval to the other, during the test. Table 37 presents the maximum, minimum and duration of hydraulic stresses during the various drill-stem tests. At no time, during any of the drill-stem tests, was a pressure response observed above or below the packed-off interval. Small pressure changes were observed that temporally coincided with the raising or lowering of the drill-stem tool to initiate or end the test. The packers, annular seals, and geologic formations appear to have adequate seals and/or low enough permeability to retard detectable fluid flow at the pressures and test durations recorded in Table 37.

The pressure responses within the packed-off interval were analyzed using Cooper et al., (1967) and Papadopulous et al., (1973). The general form of the solution is :

$$
\frac{\mathrm{H}}{\mathrm{H}_{\mathrm{o}}}=\mathrm{F}(\beta, \alpha)
$$

where

$\beta=T_{t} / r_{0}^{2}$

$\alpha \quad=r_{s}^{2} S / r_{o}^{2}$

$\mathrm{r}_{\mathrm{S}} \quad=$ effective radius of the well

$\mathrm{r}_{\mathrm{c}} \quad=$ radius of the well casing in the interval over which the head change takes place

$\mathrm{T} \quad=$ transmissivity of the well 


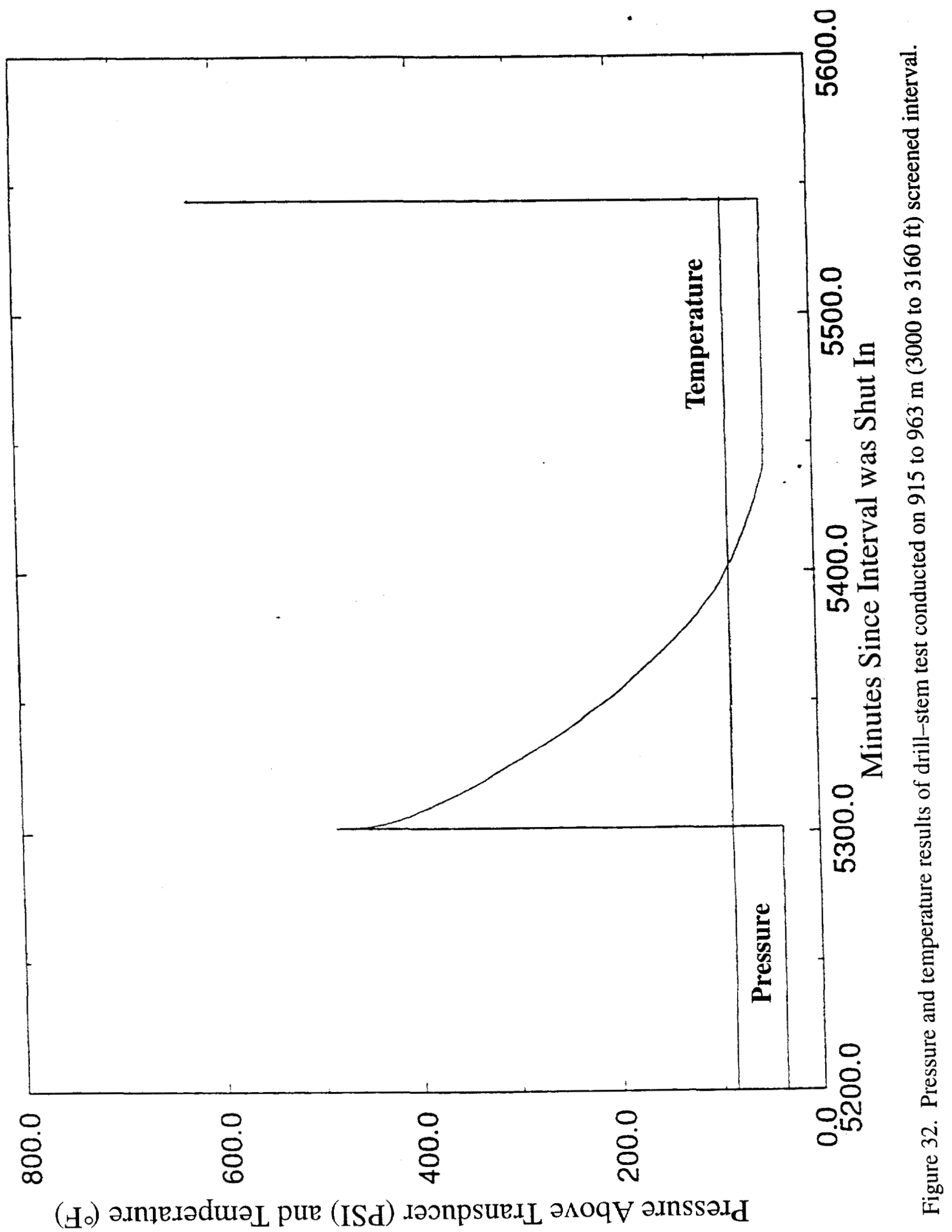




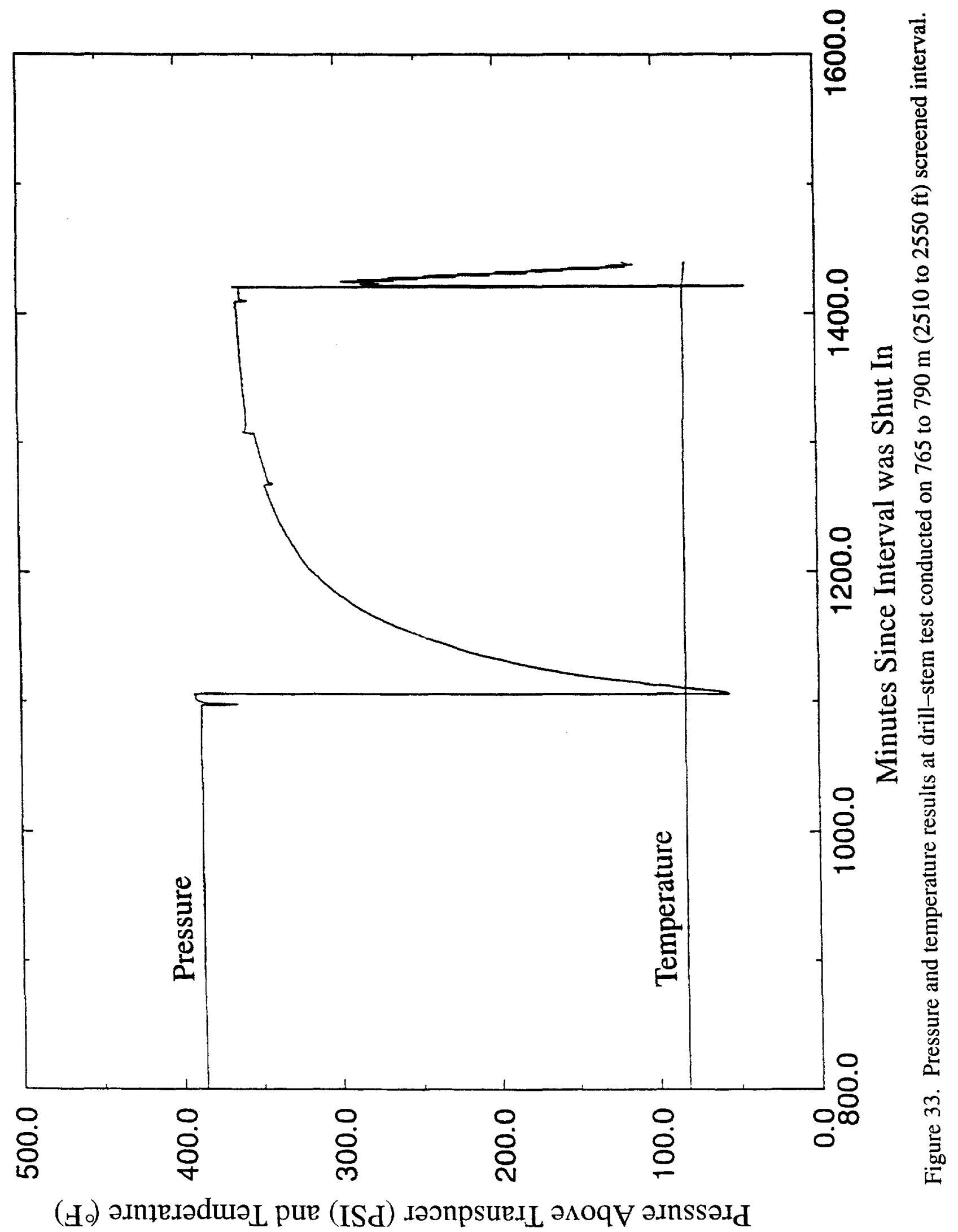




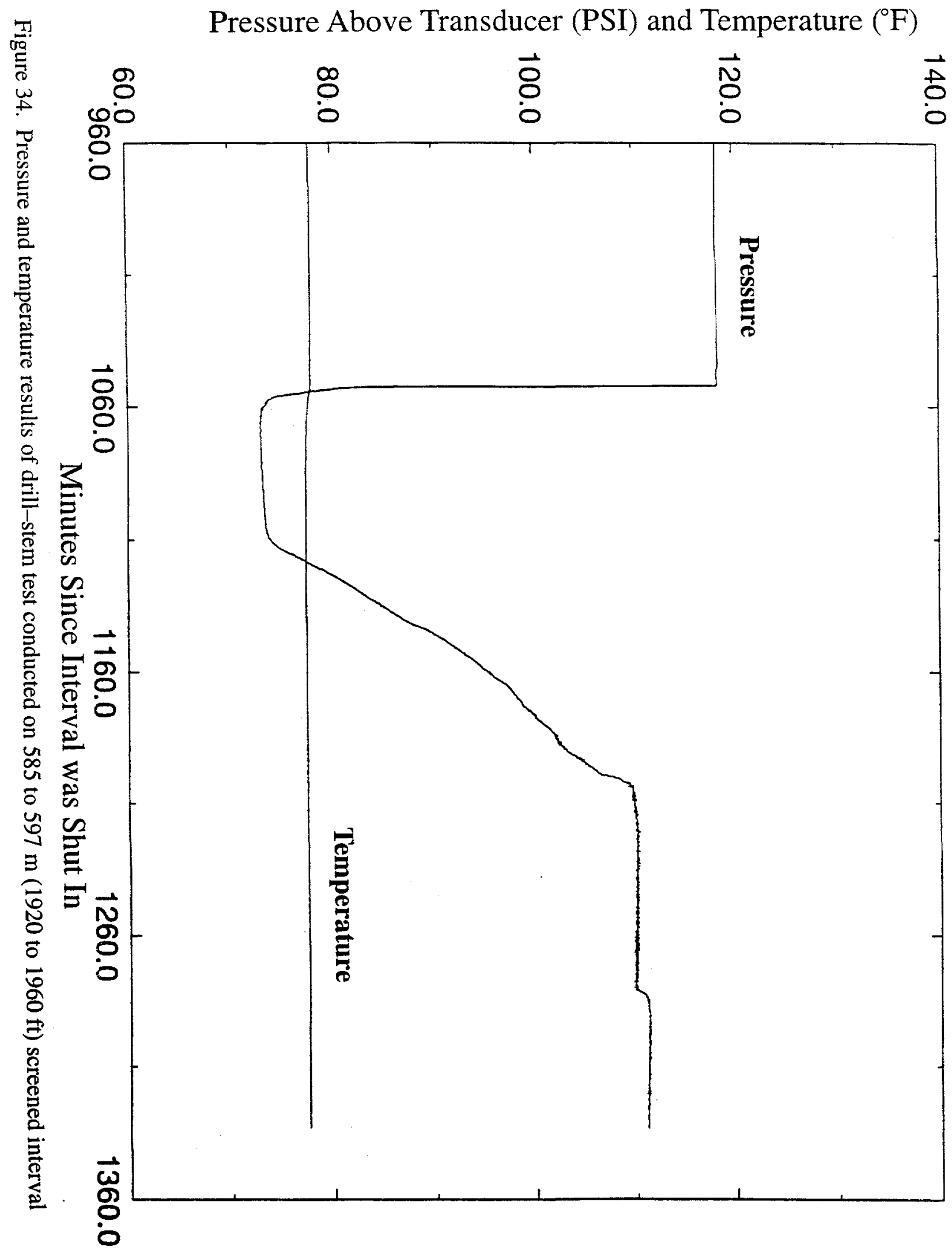


$\mathrm{t}=$ time since the instantaneous head change

$\mathrm{H}=$ head in the well at time $>0$

$\mathrm{H}_{\mathrm{O}} \quad=$ instantaneous head change in the well

$F(\beta, \alpha)=a$ function of $b$ and a whose tables and graphs are present in Cooper, et al., (1967).

The data and type curves for the drill-stem test conducted on the 915 to $963 \mathrm{~m}$ ( 3000 to 3160 $\mathrm{ft}$; lower Simonson Dolomite) screened interval are presented in Figures 35 and 36 . Results of the analysis indicate an estimated transmissivity for this zone ranging from $1 \times 10^{-5}$ to $2 \times 10^{-5} \mathrm{~m}^{2} / \mathrm{s}$. Two analyses were conducted on the data owing to the poor fit of the data to the type curves. If the value of $\alpha$ for the relevant type curve is within two orders of magnitude of the actual value, the error in the determined $\mathrm{T}$ is less than about $30 \%$ (Papadopulos et al., 1973). The data and type curves for the drill-stem test conducted on the 765 to $790 \mathrm{~m}$ ( 2510 to $2590 \mathrm{ft}$; Upper Eleana Formation) screened interval are presented in Figure 37 . Results of the analysis indicate a transmissivity of $7.5 \times 10^{-6} \mathrm{~m}^{2} / \mathrm{s}$ for this zone.

The transmissivities determined from all of the drill-stem tests are only representative of materials close to the borehole. Materials potentially impacting the test include the screen, gravel pack, and geologic formations that may have been negatively impacted through the introduction of mud during drilling or positively impacted during subsequent development efforts. In addition, subsurface conditions at ER-12-1 violate several assumptions inherent in Cooper et al., (1967): for example, the conditions of infinite areal extent, homogeneity, isotropy, uniform thickness, full aquifer penetration, and the lack of well-bore storage are not met. The transmissivities estimated by this analysis should be treated, at best, as an order of magnitude estimates. It is interesting to note that the Lower Simonson Dolomite in the screened interval of 915 to $963 \mathrm{~m}$ (3000 to $3160 \mathrm{ft}$ ) has a transmissivity equal to that determined for the composite borehole and is also under-pressured by

Table 37. Maximum, Minimum, and Duration of Stress during Drill-stem Tests Conducted at ER-12-1.

\begin{tabular}{cllc}
\hline Zone Tested & Max Stress $\mathrm{m}(\mathrm{ft})$ & Min Stress $\mathrm{m}(\mathrm{ft})$ & $\begin{array}{c}\text { Duration of } \\
\text { Stress }\end{array}$ \\
\hline $585-598 \mathrm{~m}(1920-1960 \mathrm{ft})$ & $121 \mathrm{~m}(397 \mathrm{ft})$ & $6.0 \mathrm{~m}(19.6 \mathrm{ft})$ & $149 \mathrm{~min}$ \\
$765-790 \mathrm{~m}(2510-2590 \mathrm{ft})$ & $310 \mathrm{~m}(1016 \mathrm{ft})$ & $25.3 \mathrm{~m}(82.9 \mathrm{ft})$ & $212 \mathrm{~min}$ \\
$915-963 \mathrm{~m}(3000-3160 \mathrm{ft})$ & $381 \mathrm{~m}(1250 \mathrm{ft})$ & $8.7 \mathrm{~m}(28.5 \mathrm{ft})$ & $138 \mathrm{~min}$ \\
\hline
\end{tabular}




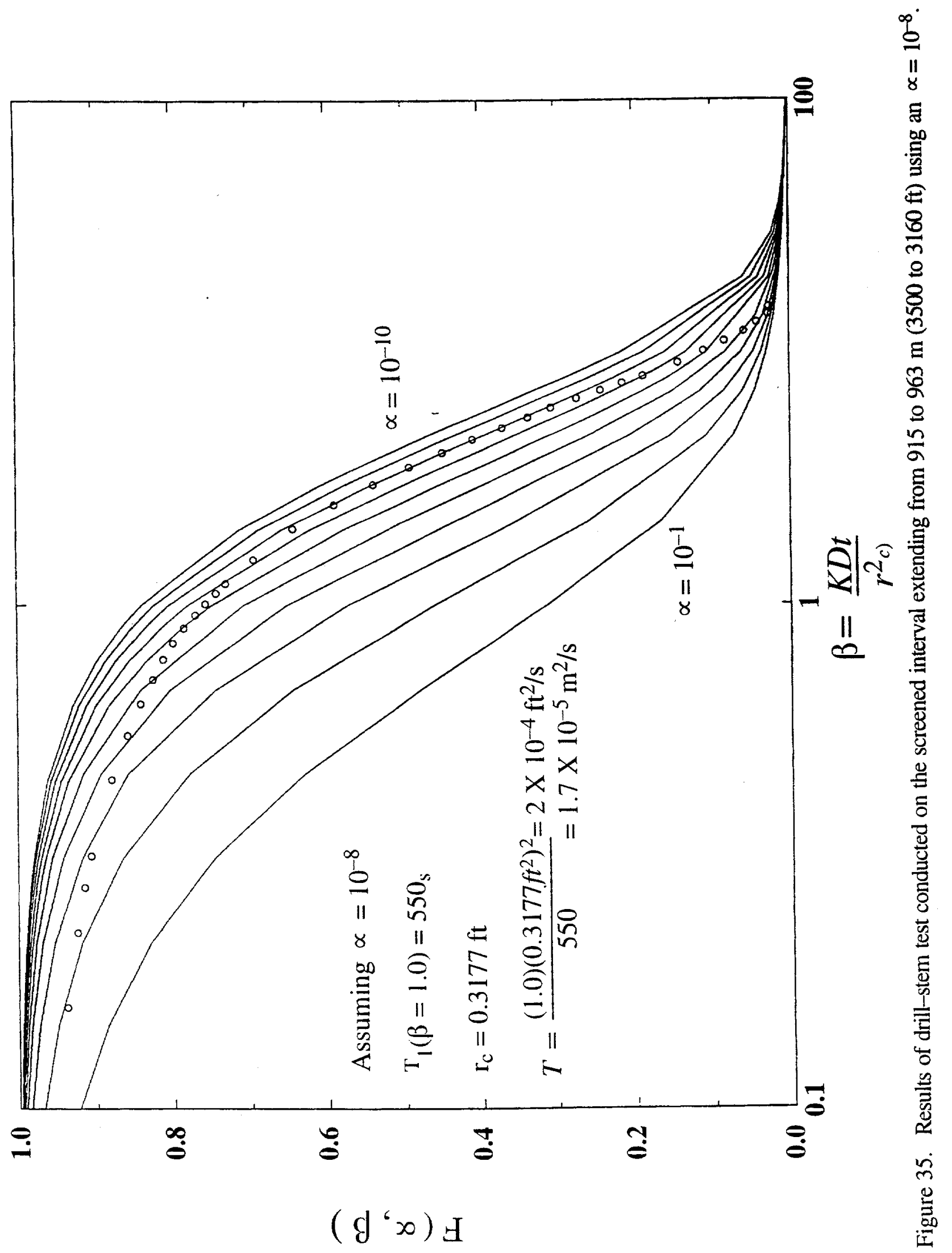




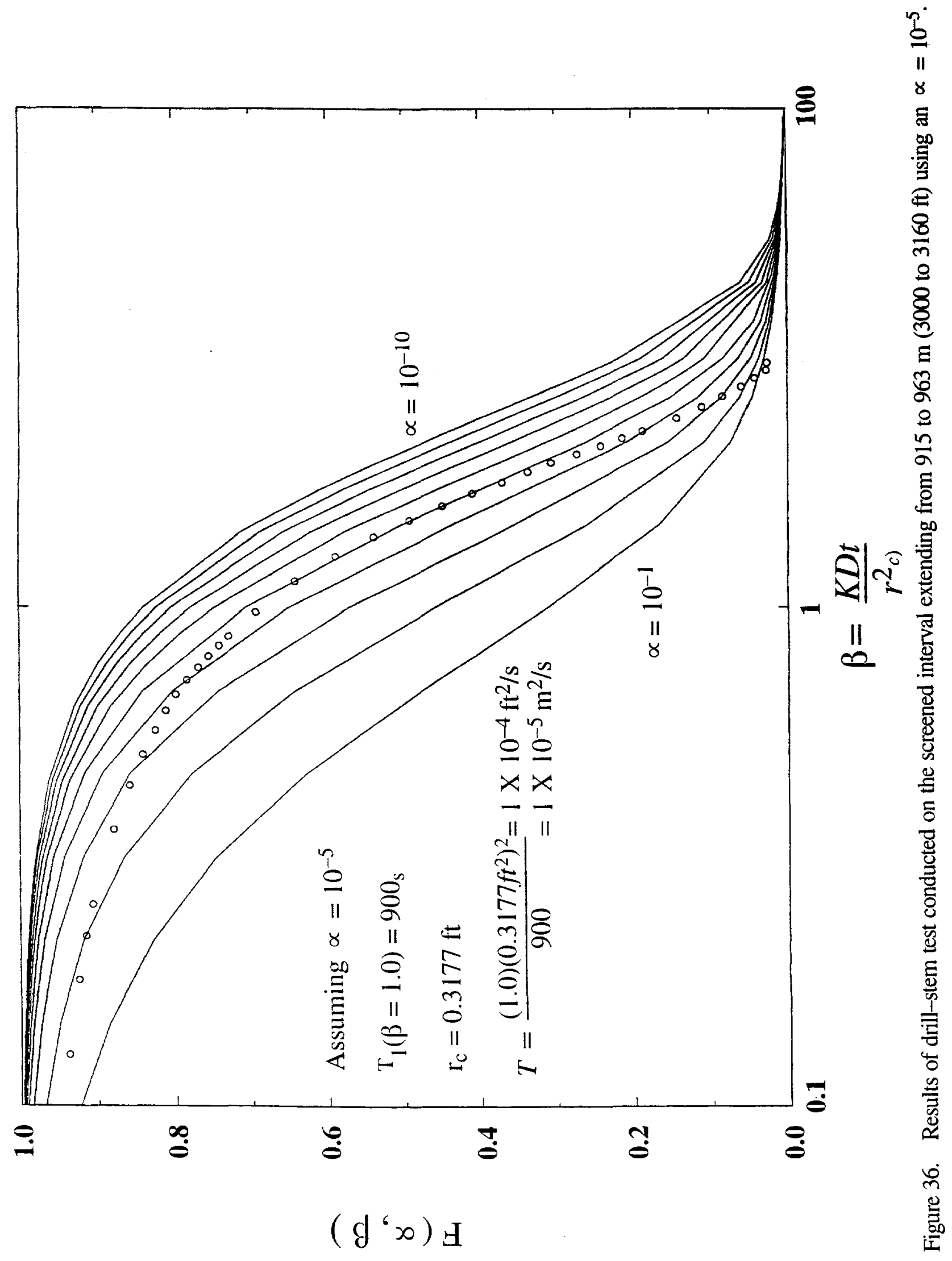




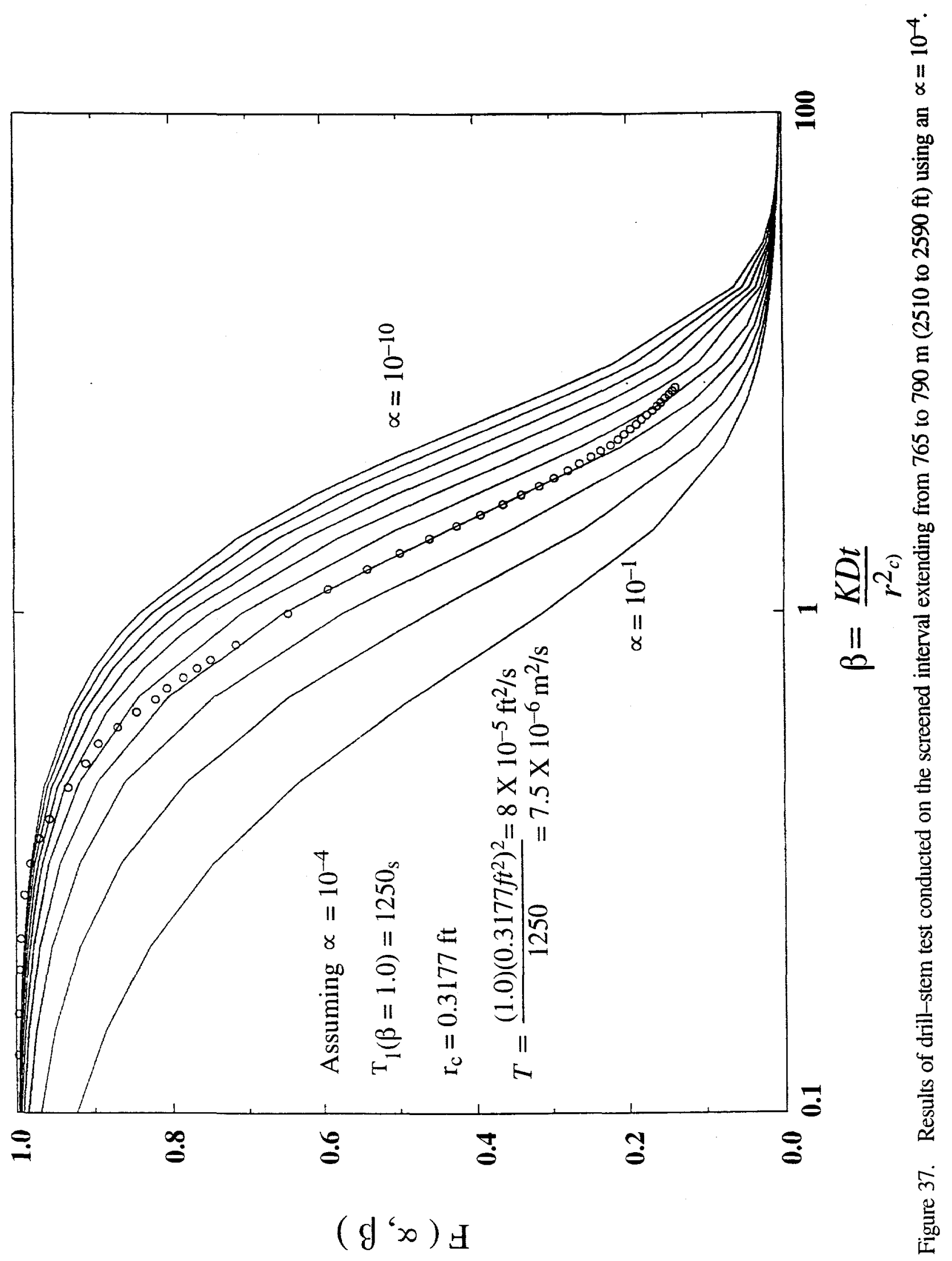


approximately $396 \mathrm{~m}(1300 \mathrm{ft})$ relative to the upper zones within the borehole. This evidence supports the interpretation of the long-term aquifer test.

The pressure response following the test conducted on 585 to $597 \mathrm{~m}$ (1920 to $1960 \mathrm{ft}$; Figure 34) was characterized by a rapid drop during depressurization once the drill-stem test tool was opened, followed by a relatively static period of approximately 45 minutes where the pressure response rose approximately $0.3 \mathrm{~m}$ (one $\mathrm{ft}$ ) of head. The relatively static period was followed by a rapid increase in pressure. The static period masked the early pressure response and prevented analysis of these data. Two potential explanations for the period of static pressures are offered; the screened zone between 585 to $597 \mathrm{~m}$ (1920 and $1960 \mathrm{ft}$ ) was subjected to various types of drilling fluids during well construction, including mud, polymers and air foam, and the drilling fluid may have temporarily plugged fractures during the initial portion of the drill-stem test, causing a slow initial rise in pressure. The sustained $14 \mathrm{~m}(45 \mathrm{ft})$ head differential may have eventually forced a channel through the filtrate, allowing a much more rapid response. An alternative explanation is the zone rapidly dewatered during the withdrawal test, exposing the transducer to air. The pressure did not rise until the zone refilled with water.

\section{Radially Converging Aquifer Test of the Upper Zone}

A 113 to $227 \mathrm{l} / \mathrm{min}$ (30 to $60 \mathrm{gpm}$ ) pump was installed in ER-12-1 on January 4, 1993, on a string of $6 \mathrm{~cm}(2-3 / 8 \mathrm{in})$ hydril tubing to a depth of $521 \mathrm{~m}(1710 \mathrm{ft})$ in preparation for sampling the 518 to $554 \mathrm{~m}$ ( 1700 to $1820 \mathrm{ft}$ ) interval. A transducer was strapped to the pump column at approximately $496 \mathrm{~m}$ (1628 feet) below ground surface to monitor pressure during pumping. A 13.94-cm string, with sliding sleeves opposite each screened interval, was emplaced in the borehole. The only sliding sleeve open in the well was the uppermost sleeve. Static water levels were at 470.2 $\mathrm{m}(1542.5 \mathrm{ft})$ just prior to the aquifer test. Pumping was initiated on January 5, 1993, at $1028 \mathrm{hrs}$ at a rate of $189 \mathrm{l} / \mathrm{min}(50 \mathrm{gpm})$. Pumping was discontinued 13 minutes later at $1041 \mathrm{hrs}$ due to a leaking union in the discharge line. The aquifer test was reinitiated at $1057 \mathrm{hrs}$. The well was not allowed to re-establish equilibrium conditions as the aquifer test was not the primary purpose for pumping the well. Pumping was discontinued at 1500 hrs on January 5, 1993.

The data collected during the aquifer test are presented in Figure 38. The data were analyzed using interpretive software (WHIP) version 3.22 by Hydro GeoChem Inc. (1988). The initial analysis entailed plotting the drawdown and recovery data in terms of drawdown below initial fluid level versus the log of total elapsed time and plotting the best fitting line through the data according to Cooper and Jacob (1946). The best fitting line was determined by least squares best fit through the data points obtained during the period of 40 to 700 minutes following the start of the test. The data collected during this time period appeared to be characterized by a straight line with positive slope. A comparison of the measured drawdown and recovery to the best fitting line indicates the early drawdown data from the aquifer test were affected by well bore storage, the latter drawdown data may have been affected by a change in aquifer thickness or a decrease in aquifer permeability at a distance, or the presence of a no-flow barrier. The comparison also indicates that the latter portion of the recovery data was affected by residual recovery due to relict drawdown created prior 


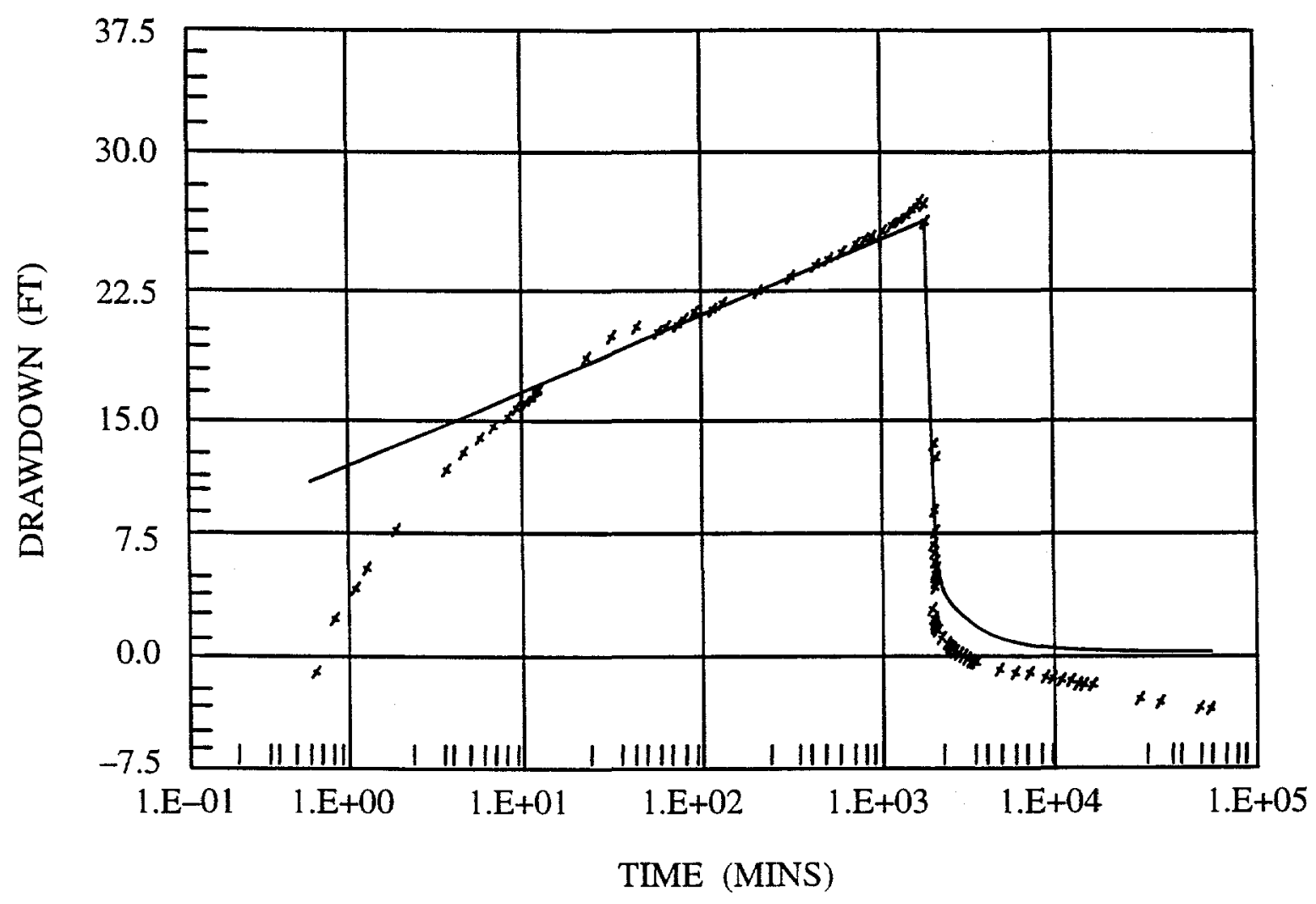

Figure 38. Reduction of ER-12-1 upper zone aquifer test, March 17, 1993.

to the start of the aquifer test. The Cooper and Jacob (1946) analysis resulted in an estimated transmissivity of $4 \times 10^{-4} \mathrm{~m}^{2} / \mathrm{s}$ and a storativity of $5 \times 10^{-3}$.

Analytical solutions were used to generate type curves that matched the observed data set. Input variables were iteratively altered with the purpose of estimating key hydrologic parameters. The results are presented in Figure 39. Type curves that utilized a transmissivity of $4.4 \times 10^{-4} \pm 2.2 \mathrm{x}$ $10^{-4} \mathrm{~m}^{2} / \mathrm{s}$, a storativity of $4.0 \times 10^{-3} \pm 0.05$, an effective casing radius (borehole storage) of 0.106 $\pm 0.03 \mathrm{~m}(0.35 \pm 0.1 \mathrm{ft})$ and a no-flow hydrologic barrier approximately $58 \pm 305 \mathrm{~m}(190 \pm 1000$ $\mathrm{ft}$ ) distant appeared to create the best fit to the observed data. The results of this analysis agree with the results of the oxygen activation $\log$ in that the screened interval between 518 to $554 \mathrm{~m}$ ( 1700 to $1820 \mathrm{ft}$ ) was the most transmissive zone within the well.

The transmissivity and storativity values estimated by this technique are very close to those determined by the Cooper and Jacob (1946) method. Washout zones exceeding a diameter of 0.76 $\mathrm{m}(30 \mathrm{in})$ in the interval of 518 to $554 \mathrm{~m}$ (1700 to $1820 \mathrm{ft}$ ), as determined by caliper runs (Figure 18), support an effective casing diameter of $0.21 \mathrm{~m}(0.70 \mathrm{ft})$ versus a calculated casing diameter of $0.12 \mathrm{~m}(0.40 \mathrm{ft})$, which takes into account the volume of the pump column and monitoring string within the well. The geologic cross section of ER-12-1 (Figure 4 from RSN geologic report) shows 


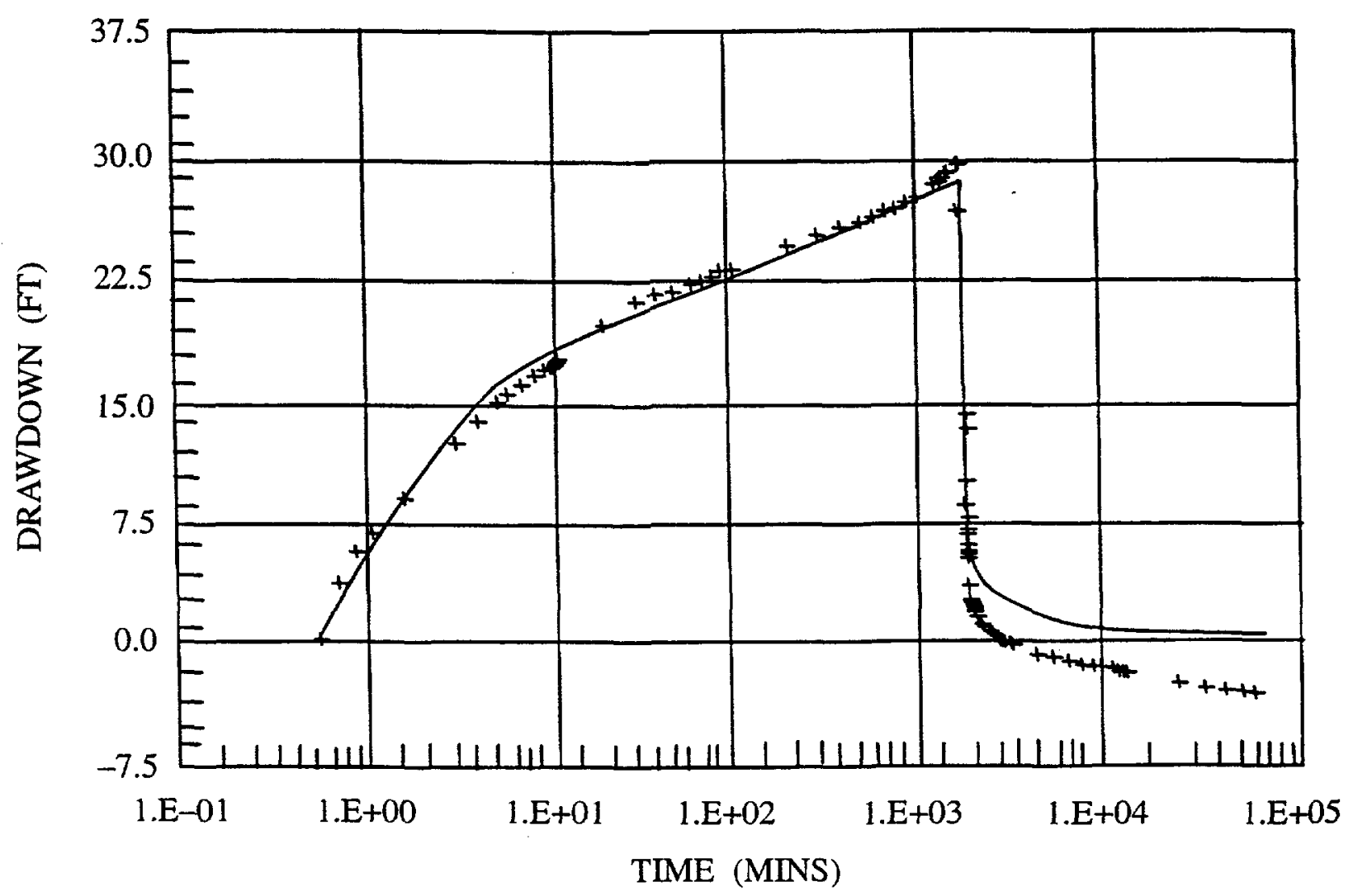

Figure 39. Hydrologic parameter estimation of ER-12-1, upper zone aquifer test, March 17, 1993.

a thin wedge of upper Simonson Dolomite or lower Guilmette Formation occurring in the test interval of 518 to $554 \mathrm{~m}$ (1700 to $1820 \mathrm{ft}$ ) and pinching out to the southeast. The decrease in thickness may be the reason for the the increase in drawdown during the latter part of the test and may also be a justification for the use of a no-flow barrier in the analytical solution.

The parameters estimated through this technique are to be treated as estimates of values associated with one potential solution to the observed data. Other solutions may also be equally valid.

\section{Hydrochemistry}

Routinely monitored samples, collected during drilling, completion, development and testing activities at ER-12-1, consisted of field measurements of geochemical parameters and geochemical samples obtained for laboratory analysis. Field measurements were obtained for tritium, bromine, turbidity, $\mathrm{pH}$, and temperature. Geochemical samples were routinely obtained during drilling and well development for metals and hydrocarbon analysis to asses the quality of fluids introduced to, and removed from, the well. Geochemical characterization samples were obtained at the conclusion of the upper zone aquifer test. 


\section{Field Measurements During Drilling}

Field measurements for tritium were conducted whenever fluids were introduced into, or removed from, the well. Field tritium measurements prior to September 1992, were made using a 1206 BetaMan liquid scintillation counter. After September 1992, a Packard liquid scintillation counter was used for tritium field measurements. All drilling fluids brought onto location were measured for background tritium levels. Returns from the well during drilling were monitored hourly. Fluids recovered from the well during development and testing activities were also monitored hourly for tritium content. All tritium field measurements obtained at ER-12-1 indicated tritium contents well below the $20,000 \mathrm{pCi} /$ State of Nevada Safe Drinking Water (SNDW) standard. No detectable trends in tritium concentration were observed (Figure 40).

A concentration of approximately $21 \mathrm{mg} / \mathrm{l}$ of lithium bromide was added to all fluids introduced to ER-12-1 during drilling as a hydrologic tracer. Bromine concentrations were monitored in fluids produced from the well to determine the degree to which development had occurred. Bromine measurements were obtained in the field using an Orion 250A meter with an Orion bromine probe and reference cell.

During drilling, bromine field measurements were obtained for drilling fluids held in Baker-tanks prior to use, and for returns from the well (Figure 41). While using air-foam as the circulation medium, there appeared to be a definite dilution in the bromine concentration of returned fluids following penetration of the static water level within the well between 450 and 520 meters (1500 and $1700 \mathrm{ft}$ ). However, after converting to mud as the circulation medium, the bromine concentrations in the Baker-tank and in the returns from the well became more consistent. This is expected as the weight of the drilling mud does not allow formation fluids to enter the wellbore. Bromine concentrations within the Baker-tanks and in returns from the well were consistently less than the target concentration of $21 \mathrm{mg} / \mathrm{l}$. Laboratory experiments at DRI determined that bentonite adsorbed bromine until mixed with the detergent component of the air-foam mixture. Depressed concentrations of bromine in the baker tanks were most likely due to inadequate mixing of bentonite and detergent or adsorption to bentonite if drilling mud was being used.

\section{Field Measurements During Well Development and Testing}

Tritium field measurements obtained from fluids produced from the well were monitored by DRI personnel. Tritium field measurement results were compared with tritium measurements for UE-16d (essentially tritium-free water) $10,000,20,000$ and 40,000 pCi/l standards (Figure 42). All samples were found to be well below the $20,000 \mathrm{pCi} / \mathrm{l}$ SNDW. Temporal trends in the tritium activity of the fluids from ER-12-1 were virtually identical (with one exception) to temporal changes observed in the baseline (UE-16d water) and $20,000 \mathrm{pCi} / \mathrm{L}$ standards. The only relative difference in Figure 42 were early tritium measurements (derived from water produced during initial well development) invariably plotted below the UE-16d (dead) water. Once aquifer testing was initiated $(625,000 \mathrm{~L}$ or 165,000 gallons had been discharged from the well), fluids produced from well ER-12-1 were coincident with UE-16d (dead) water activities. The change in ER-12-1 tritium activity relative to $U E-16 \mathrm{~d}$ water activity may have been due to minor contamination of the UE-16d 


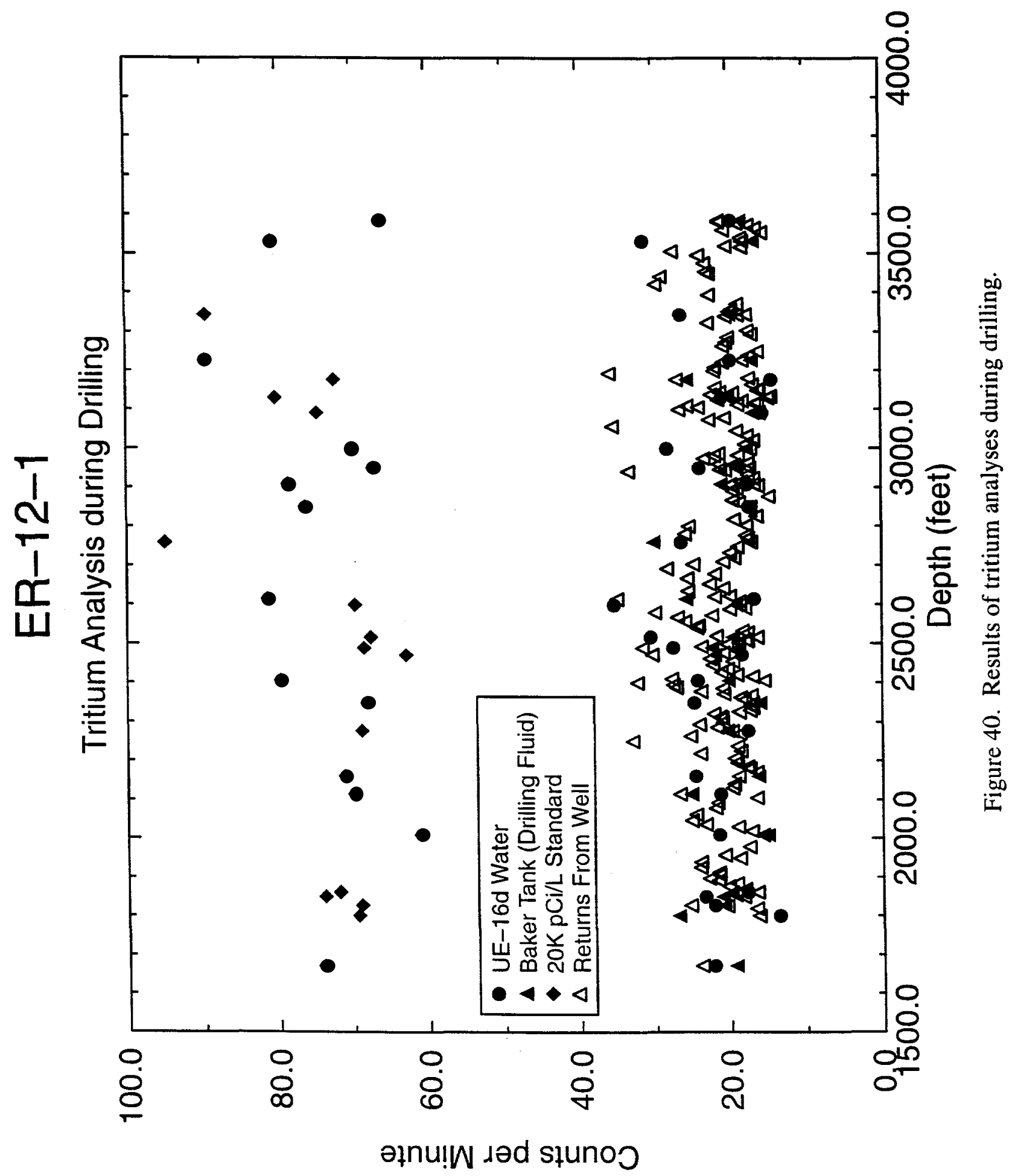




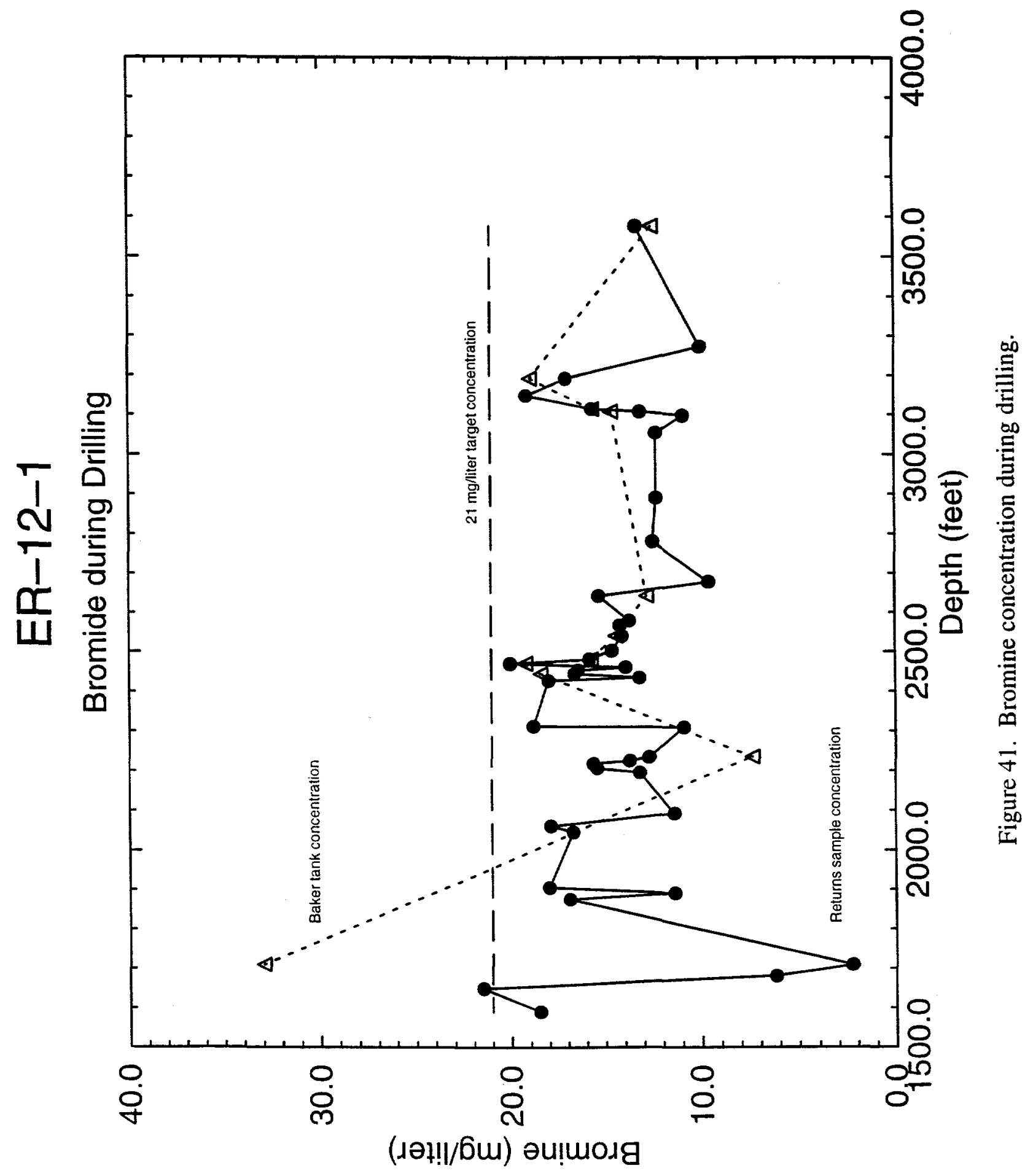




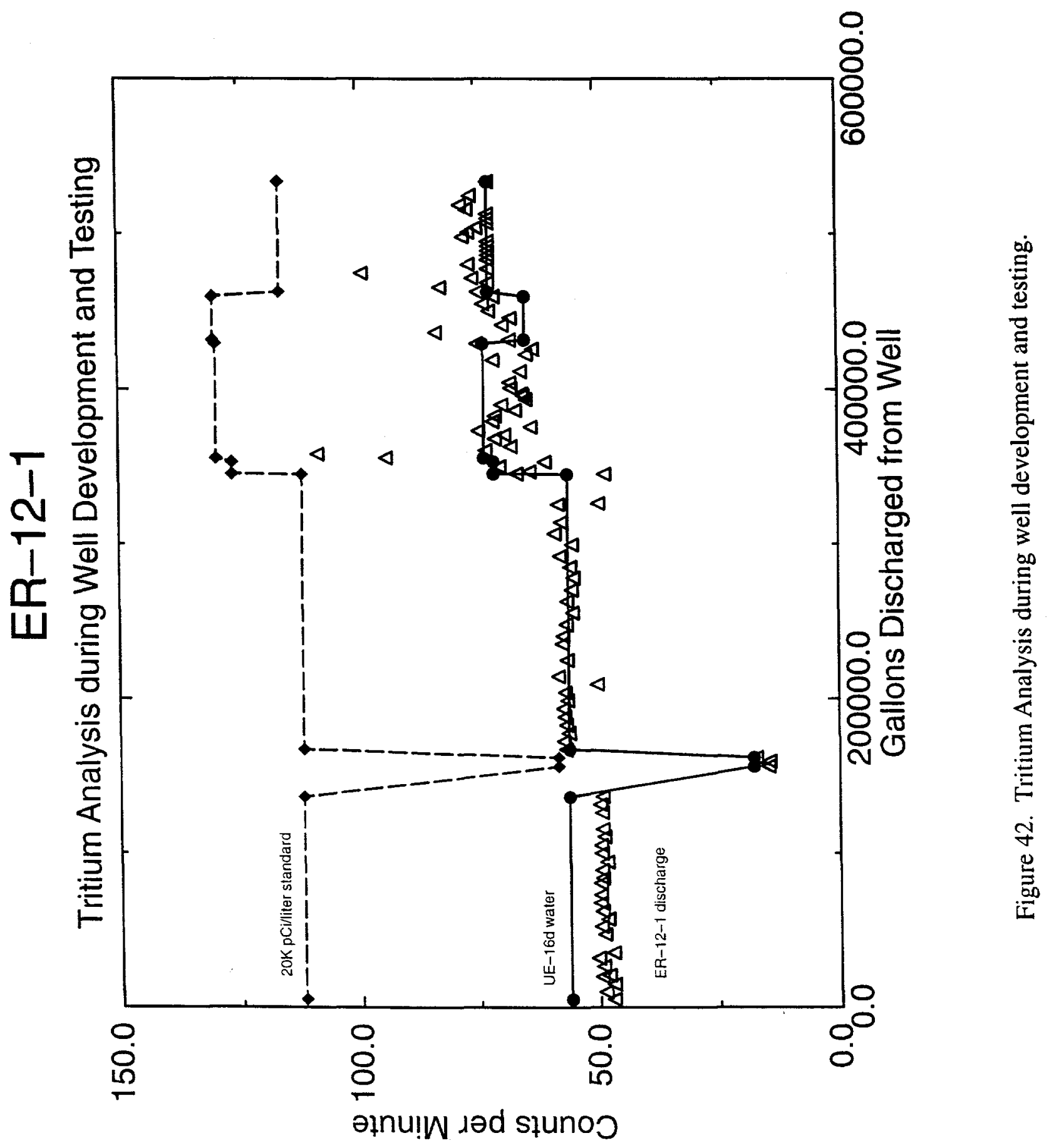


sample during initial well development, a decrease in the production of drilling fluids injected into the well that may have had a relatively less tritium activity than groundwater surrounding well ER-12-1, or a minor increase in tritium concentrations in the ER-12-1 water during initial aquifer testing due to contaminant migration from tritiated fluids that may have recharged through the nearby U12e tunnel pond sumps (located $450 \mathrm{~m}$ to the east of well ER-12-1). Relative temporal variations observed in the baseline (UE-16d water) and the $20,000 \mathrm{pCi} / 1$ standard displayed in Figure 42 were due to the use of different scintillation counters, scintillation solutions, and possibly erroneously high sample counts due to failure to sufficiently dark-adapt samples prior to counting. An example is the sharp increase in all standards and samples at $1.26 \times 10^{6} \mathrm{~L}$ discharged $(335,000$ gallons). This increase is due to the change in field scintillation counters from the 1206 Betaman to the Packard liquid scintillation counter.

Bromine concentrations during well development and testing are displayed in Figure 43. During initial well development, bromine concentrations in recovered fluids displayed a logarithmic decrease from approximately $18 \mathrm{mg} / \mathrm{l}$ to between 2.5 and $3 \mathrm{mg} / \mathrm{l}$. Spiking in the bromine concentration of the discharge occurred during the open-hole aquifer test. An abrupt increase, to approximately $20 \mathrm{mg} / \mathrm{l}$, resulted from the introduction of additional fluids to the well during the second phase of well development. Subsequent removal of fluids by air lifting resulted in a reduction of bromide concentration in recovered fluids to approximately $9 \mathrm{mg} / 1$.

Prior to placement of the $0.14 \mathrm{~m}$ (5-1/2 in) casing and sliding sleeves, all five completed zones were capable of contributing to the production of fluids removed from the well. Following placement of the $0.14 \mathrm{~m}(5-1 / 2 \mathrm{in})$ casing the lower four sliding sleeves were closed, isolating those zones from the well. The abrupt drop in bromine concentrations from approximately $9 \mathrm{mg} / \mathrm{l}$ to about $3 \mathrm{mg} / \mathrm{l}$ was due to the change from recovering fluids from all five zones to only the uppermost zone producing the fluids recovered from the well. The upper zone was the most transmissive and had one of the highest heads in the well. A greater amount of water had been produced from this zone than any other; thus, the fluids from this well would tend to have the lowest concentration of bromine.

Field measurements for the $\mathrm{pH}$ of the fluids recovered from the well were obtained during pumping of the well using an Orion $250 \mathrm{~A}$ meter and Orion Triode ( $\mathrm{pH} /$ temperature/reference cell) probe. $\mathrm{pH}$ measurements were not obtained during development of the well by air-lifting due to the potential alteration of the $\mathrm{pH}$ by aeration.

$\mathrm{pH}$ measurements during initial well development displayed a logarithmic decrease from about 9.2 units to an average of 7.55 units (Figure 44). The $\mathrm{pH}$ of recovered fluids again stabilized, during initial aquifer testing, at approximately 7.5 units. Near the end of both stages of pumping, some increased $\mathrm{pH}$ values were obtained. These higher $\mathrm{pH}$ values may be the result of measurement errors, or possibly result from drawdown in the well allowing contributions from lower pressure zones which would not normally contribute fluid to the well. The $\mathrm{pH}$ of recovered fluids averaged approximately 7.3 units during aquifer testing of the upper zone only. 


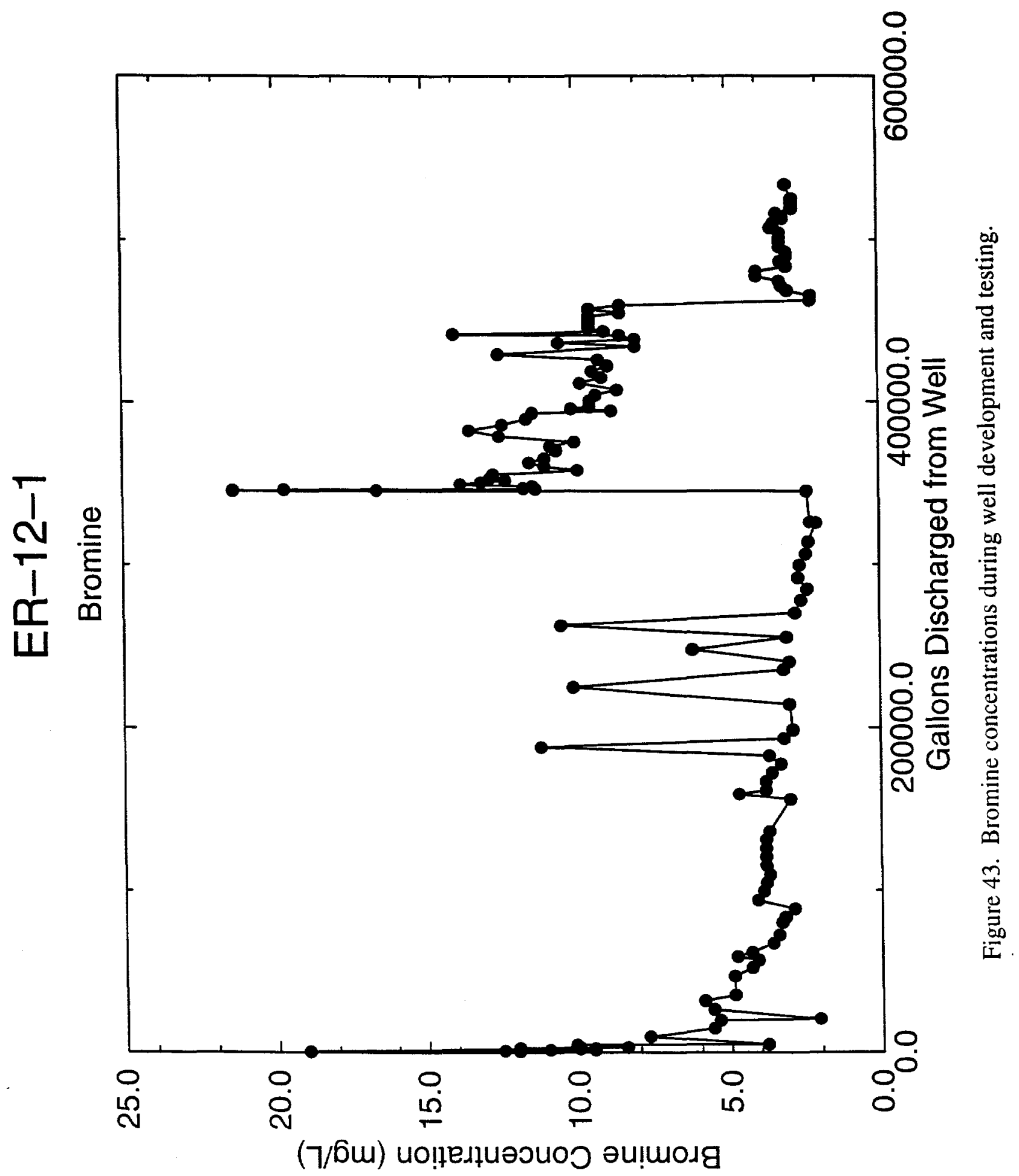


Temperature measurements, although obtained when $\mathrm{pH}$ measurements were taken using the temperature probe included in the Orion $250 \mathrm{~A}$ meter, should not be considered valid except during the aquifer test of the upper interval. Representative water temperature measurements were difficult to obtain due to the well head configuration during initial well development and aquifer testing. During the aquifer test of the upper interval, a valve was installed in the pump tubing to permit the acquisition of samples for temperature measurements. However, even with this improved system at least four measurements are obviously lower than the actual discharge water temperature (Figure 45).

Turbidity measurements were obtained for fluids produced from the well during development by air-lifting and during aquifer testing of the upper interval (Figure 46). The initial turbidity of fluids recovered during air-lifting exceeded the 200 NTU (Nephelometric Turbidity Unit) capacity of the HF Scientific Turbidity Meter used to obtain turbidity measurements. However, by the completion of development activities, turbidity values had fallen to approximately 40 NTU. During aquifer testing of the upper interval, turbidity values displayed a logarithmic decrease from 17.5 NTU after the first hour of pumping to approximately 3 NTU at the end of the test.

Electrical conductivity measurements were obtained for fluids produced from ER-12-1 only during aquifer testing of the upper interval. A YSI Model-33 conductivity meter was used to obtain fluid conductivity measurements. A marked increase in fluid conductivity was observed for the first five measurements obtained during the aquifer test. Following the initial increase from approximately $1080 \mu \mathrm{mhos} / \mathrm{cc}$, fluid conductivity averaged about $1160 \mu \mathrm{mhos} / \mathrm{cc}$ for the remainder of the pumping portion of the aquifer test (Figure 47).

\section{Field Measurement Quality Control/Quality Assurance}

All field measurements (tritium, bromide, $\mathrm{pH}$, turbidity, etc.) acquired by DRI personnel during drilling, development or testing activities at ER-12-1 were obtained using DRI Standard Operating Procedures and Prompt Sheets. Standard Operating Procedure, governing a particular method, dictated the method for instrument calibration and data acquisition based on manufacturer specifications. Prompt Sheets containing instrument calibration and measurement data were completed in the field. Results of measurements were also recorded in the ER-12-1 Field Activity Logbook. As soon as possible, following completion of individual field measurements, the Prompt Sheets were archived in files maintained at the DRI office in Las Vegas, Nevada.

\section{Geochemical Water-Quality Samples}

A total of 12 geochemical samples were obtained in association with activities at ER-12-1. These 12 samples are described in Table 38. Samples 10001 through 10009 were obtained to evaluate the hydrochemical characteristics of fluids introduced to, or removed from, the well during well construction. Sample 10010 was obtained to verify compliance with SNDW standards prior to discharging fluids to land surface during aquifer testing. Sample 10011 was collected at the conclusion of the initial aquifer test as a check of fluids discharged to land surface during testing. Sample 10012 was obtained for geochemical characterization at the conclusion of pumping during 


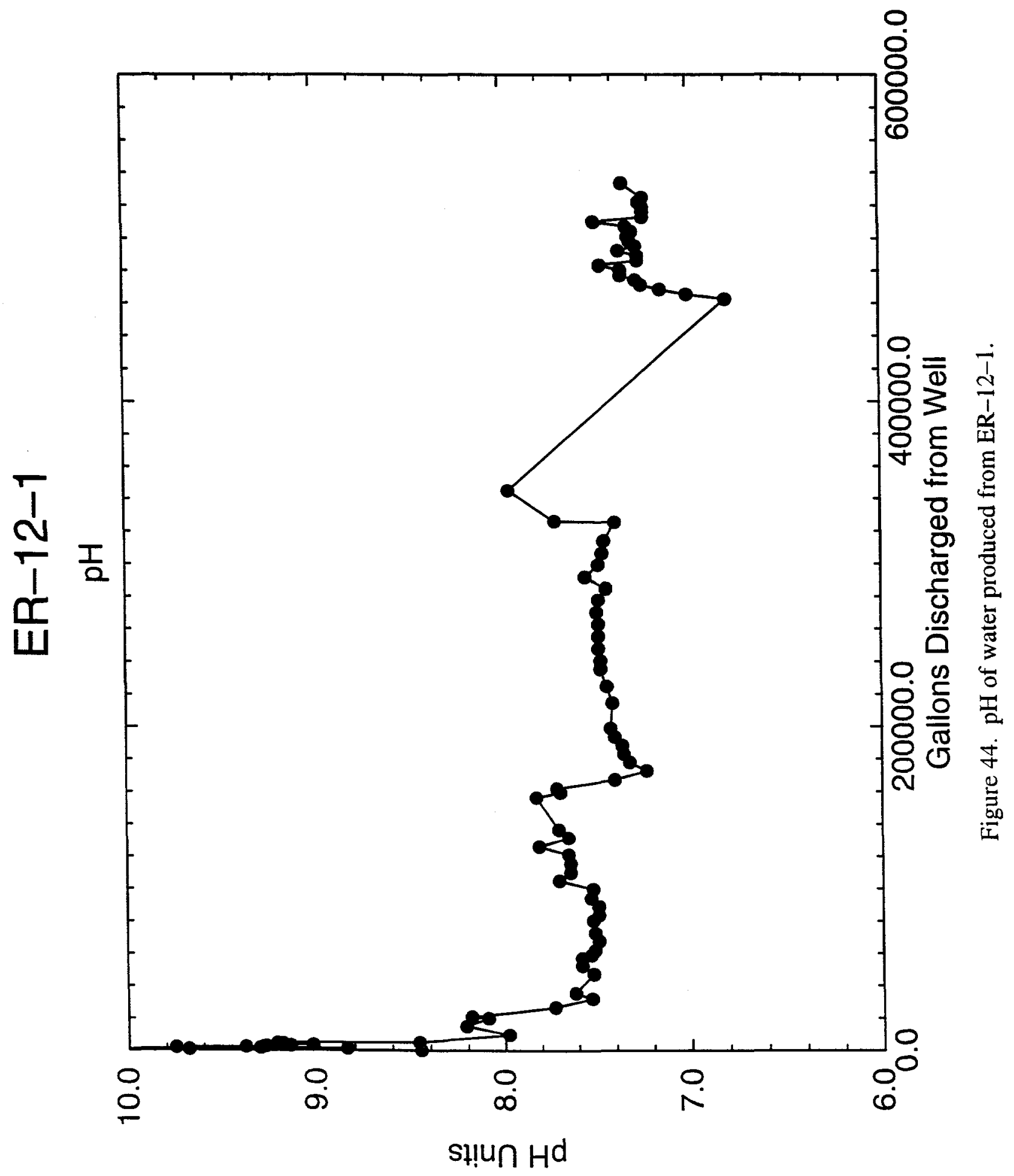




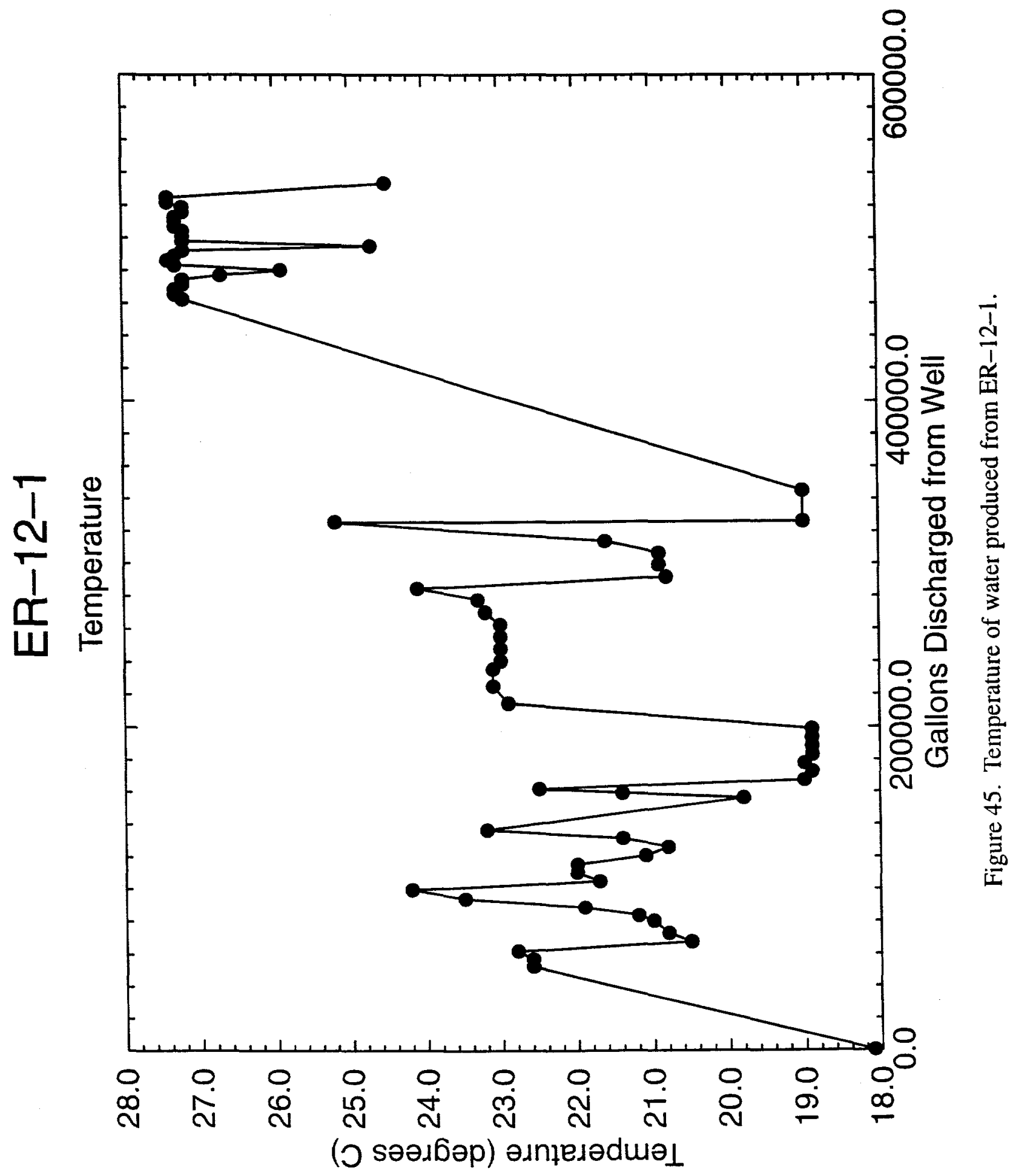




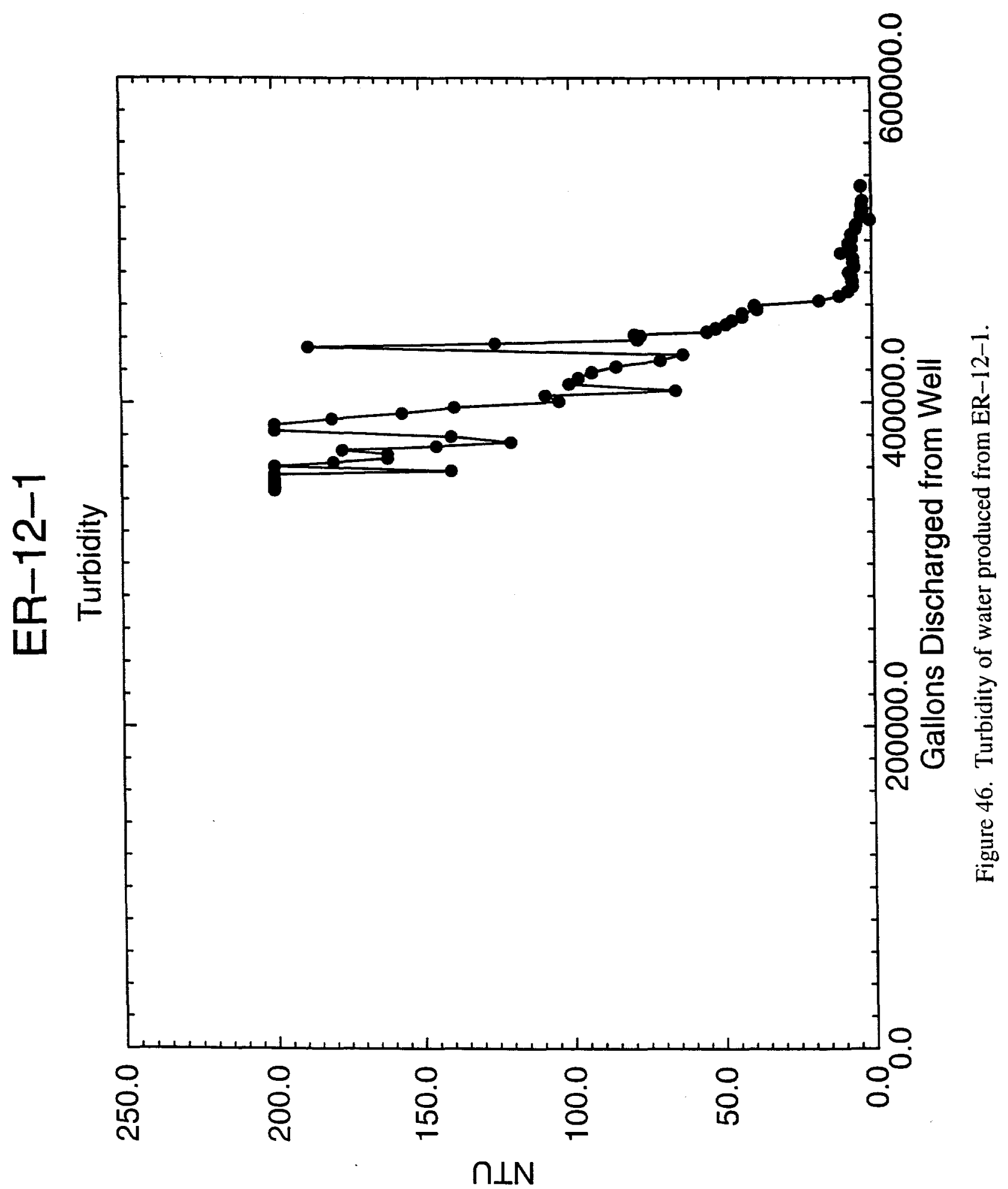




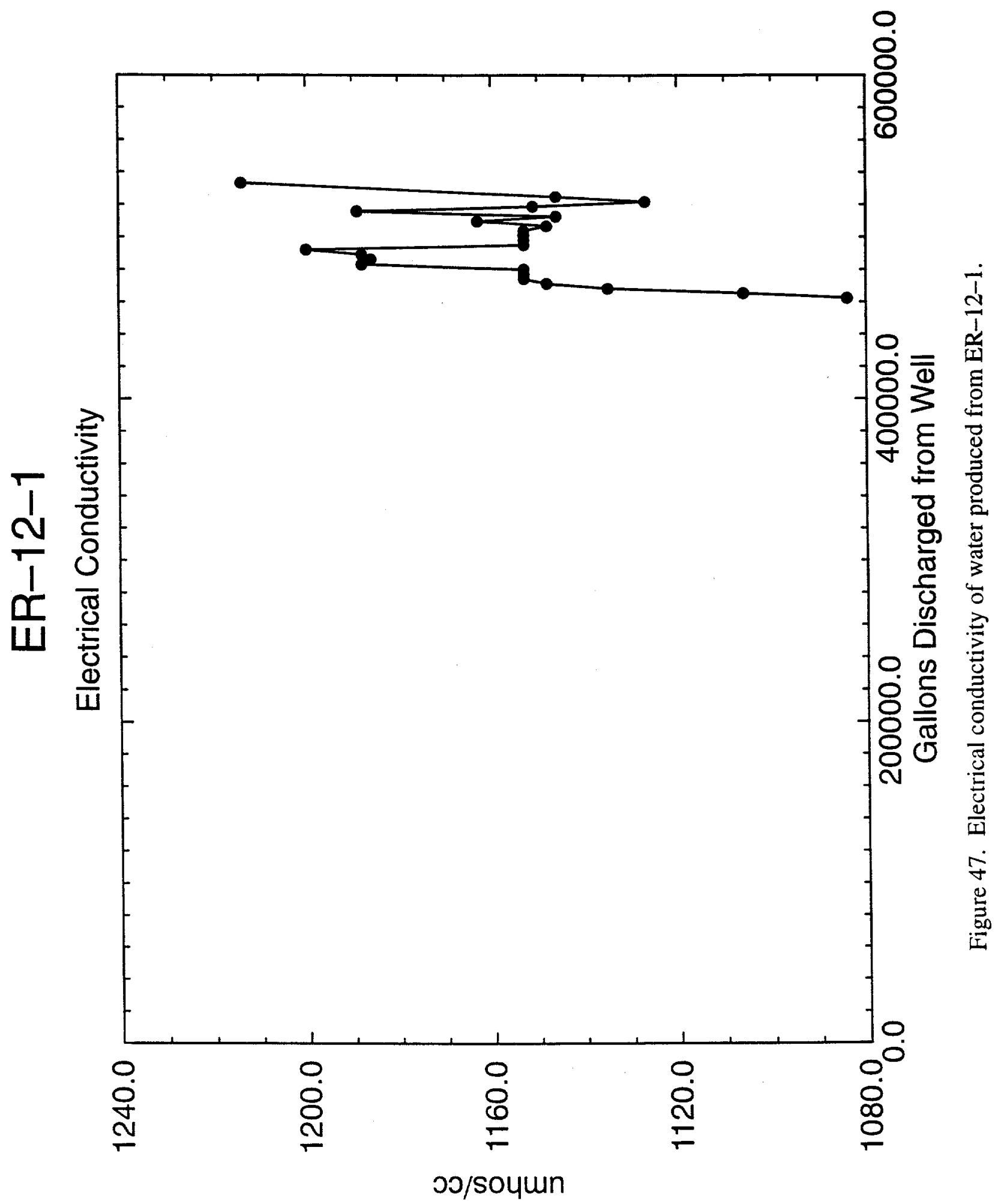


Table 38. Geochemical Samples Acquired from Well ER-12-1.

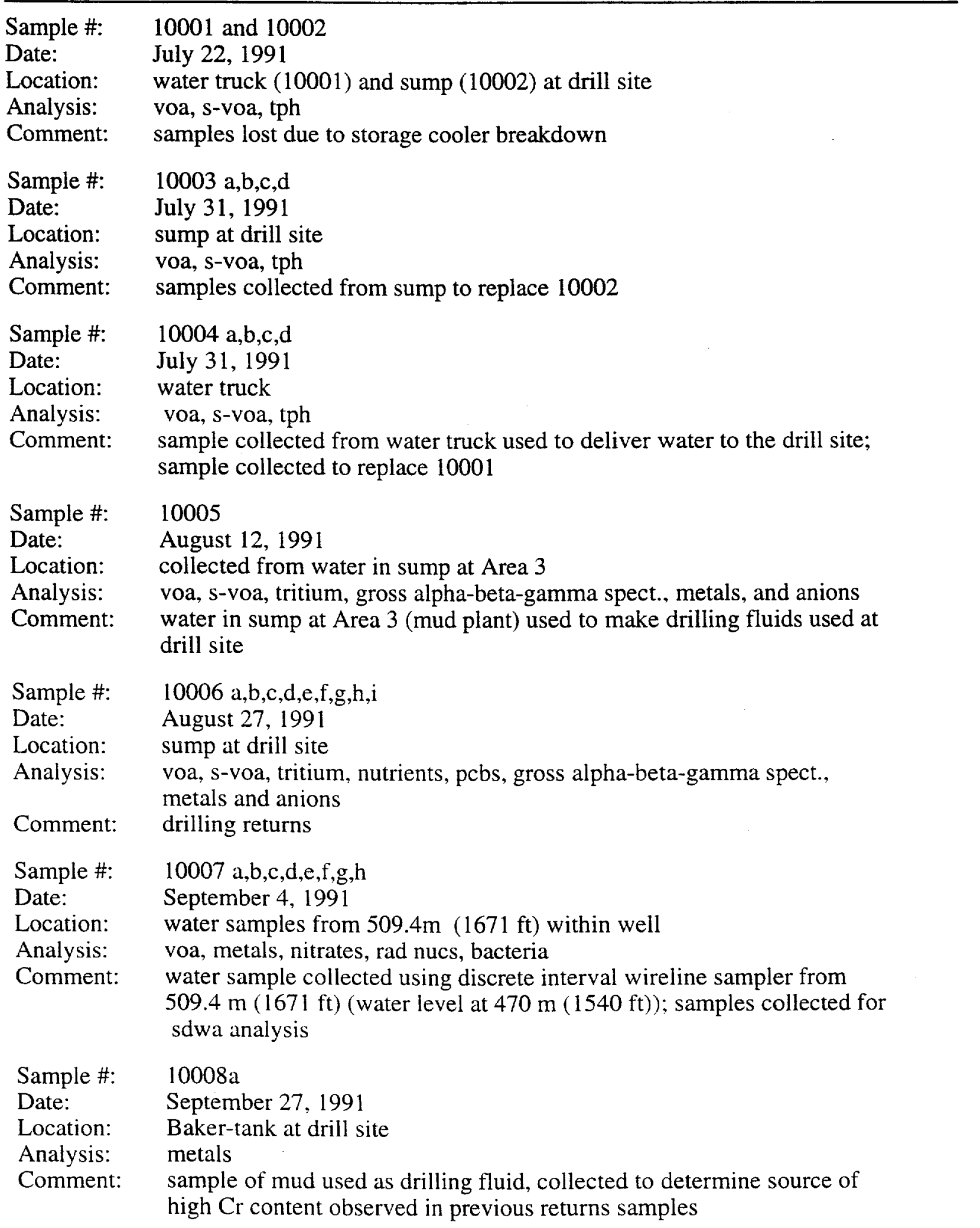


Table 38. Geochemical Samples Acquired from Well ER-12-1 (continued).

\begin{tabular}{|c|c|}
\hline $\begin{array}{l}\text { Sample \#: } \\
\text { Date: } \\
\text { Location: } \\
\text { Analysis: } \\
\text { Comment: }\end{array}$ & $\begin{array}{l}\text { 10008b } \\
\text { September } 27,1991 \\
\text { Area } 3 \text { mud plant } \\
\text { metals } \\
\text { sample of foam used as drilling fluid, collected to determine source of } \\
\text { high Cr content observed in previous returns samples }\end{array}$ \\
\hline $\begin{array}{l}\text { Sample \#: } \\
\text { Date: } \\
\text { Location: } \\
\text { Analysis: } \\
\text { Comment: }\end{array}$ & $\begin{array}{l}10009 \text { a } \\
\text { October } 11,1991 \\
\text { standpipe at drill rig } \\
\text { total petroleum hydrocarbons } \\
\text { sample of drilling mud collected immediately prior to entering well }\end{array}$ \\
\hline $\begin{array}{l}\text { Sample \#: } \\
\text { Date: } \\
\text { Location: } \\
\text { Analysis: } \\
\text { Comment: }\end{array}$ & $\begin{array}{l}10009 \mathrm{~b} \\
\text { October } 11,1991 \\
\text { flowline at drill rig } \\
\text { total petroleum hydrocarbons } \\
\text { sample of drilling fluids collected immediately after return to surface } \\
\text { from well }\end{array}$ \\
\hline $\begin{array}{l}\text { Sample \#: } \\
\text { Date: } \\
\text { Location: } \\
\text { Analysis: } \\
\text { Comment: }\end{array}$ & $\begin{array}{l}10010 a \\
\text { March 15, } 1992 \\
\text { pumped discharge from well } \\
\text { voa, s-voa, nitrates, rad nucs, metals, anions } \\
\text { sdwa samples collected at end of well development by pumping }\end{array}$ \\
\hline $\begin{array}{l}\text { Sample \#: } \\
\text { Date: } \\
\text { Location: } \\
\text { Analysis: } \\
\text { Comment: }\end{array}$ & $\begin{array}{l}10010 \mathrm{~b} \\
\text { March } 15,1992 \\
\text { as above } \\
\text { as above } \\
\text { as above; } 10010 \mathrm{~b} \text { collected as duplicate sample for } 10010 \mathrm{a}\end{array}$ \\
\hline $\begin{array}{l}\text { Sample \#: } \\
\text { Date: } \\
\text { Location: } \\
\text { Analysis: } \\
\text { Comment: }\end{array}$ & $\begin{array}{l}10011 \\
\text { April } 1,1992 \\
\text { pumped discharge from well } \\
\text { cations, anions, nitrate, rad nucs and } \mathrm{Sr} \\
\text { samples collected at end of aquifer testing ( } \mathrm{Sr} \text { collected for USGS) }\end{array}$ \\
\hline $\begin{array}{l}\text { Sample \#: } \\
\text { Date: } \\
\text { Location: } \\
\text { Analysis: } \\
\text { Comment: }\end{array}$ & $\begin{array}{l}10012 \\
\text { January 6, } 1993 \\
\text { pumped discharge from well } \\
\text { complete geochemical sample suite } \\
\text { samples collected at end of aquifer test on upper zone }\end{array}$ \\
\hline
\end{tabular}


the upper-zone aquifer test. All produced fluids were contained in the lined sump at the ER-12-1 well site during aquifer testing of the upper zone.

\section{Samples 10001 and 10002}

Samples 10001 and 10002 were collected at 1400 hours on July 21,1991 to assess the quality of water introduced to, and removed from the well during construction. Sample 10001 was collected from the water truck delivering water to be used in the drilling operation at the drilling location, and taken to the REECo laboratory in Mercury for volatile organic analysis (VOA), semi-volatile organic analysis (SVOA), tritium analysis and gross alpha-beta-gamma spectroscopy. Water Well 8 at the NTS was the source of this water. Sample 10002 was collected from the sump at the drilling location and is comprised of drilling returns from the well. This sample was collected for VOA, SVOA, and total petroleum hydrocarbons (TPH) analysis. Due to the failure of the refrigeration unit at the REECo laboratory, all samples were lost except those collected for tritium and gross alpha-beta-gamma spectroscopy from the water truck (sample 10001).

Tritium concentration in sample 10001 was below the detection limit of the analysis method used, i.e., less than $307 \mathrm{pCi} / \mathrm{l}$. The SNDW standard for tritium is $20,000 \mathrm{pCi} / 1$. Gross alpha radiation was reported to be $2.87 \mathrm{E}-9 \mu \mathrm{Ci} / \mathrm{ml}$, slightly above the detection limit of $2.15 \mathrm{E}-9 \mu \mathrm{Ci} / \mathrm{ml}$. This activity is well within the SNDW standard of $15.0 \mathrm{E}-9 \mu \mathrm{Ci} / \mathrm{l}$. Gross beta activity was below the detection limit of $2.73 \mathrm{E}-9 \mu \mathrm{Ci} / 1$. The state of Nevada does not set a drinking water standard for beta radiation. Gamma activity was not detected in the sample.

\section{Samples 10003 and 10004}

Samples 10003 and 10004 were collected at 1520 hours on July 31, 1991 (replacing samples 10001 and 10002, described above) to determine if organic compounds were entering the circulation system at ER-12-1. Sample 10003 was collected from the sump; sample 10004 was collected from the water truck (construction water from Water Well 8). Volatile organic analyses of samples 10003 and 10004 indicated the presence of methylene chloride at an estimated concentration of $4 \mu \mathrm{g} / \mathrm{l}$. (Estimated concentrations indicate the presence of a compound that meets the identification criteria, but the result is less than the sample quantification limit of the method used.) An unknown volatile organic compound with a retention time of 1.07 minutes in the gas chromatograph was also detected at an estimated concentration of $140 \mu \mathrm{g} / \mathrm{l}$ in sample 10003 and $28 \mu \mathrm{g} / \mathrm{l}$ in sample 10004 (at a retention time of 1.09 minutes). The methylene chloride was detected in the associated laboratory blank at an estimated concentration of $1.7 \mu \mathrm{g} / \mathrm{l}$. The unknown volatile organic was not detected in the associated laboratory blank. The detection of methylene chloride in the associated laboratory blank, although at a lower estimated concentration than in the sample, cast doubt on the validity of its detection in the samples. The source and the nature of the unknown volatile organic is not known; however, due to its occurrence at a low concentration it is not considered cause for significant concern. Possible sources for this compound may be materials used in the well, the water truck, bottles used to transport samples, or laboratory contamination.

Semi-volatile organic analysis of sample 10003 indicated the presence of di-n-butylphthalate,

butylbenzylphthalate, and bis(2-ethylhexy)phthalate, at estimated concentrations of $0.8,3.0$, and 
$1.0 \mu \mathrm{g} / \mathrm{l}$, respectively. Three unknown SVOA compounds with retention times of 8.36 (tentatively identified as 4-methyl-2-pentanon-4-ol), 8.73 and 11.84 minutes and concentrations of 8,15 and 143 $\mu \mathrm{g} / \mathrm{l}$ respectively, were also detected. SVOA analysis of sample 10004 also indicated the presence of di-n-butylphthalate, butylbenzylphthalate, and bis(2-ethylhexy)phthalate at estimated concentrations of $0.7,1.0$, and $2.0 \mu \mathrm{g} / \mathrm{l}$, respectively. Six unknown VOA compounds with retention times of 8.31 (tentatively identified as 4-methyl-2-pentanon-4-ol), 9.59, 13.54, 13.89, 16.77, and 38.83 minutes at concentrations of $8,9,17,10,14$, and $8 \mathrm{ug} / 1$, respectively, were also detected. All three of the known VOA compounds found in both samples 10003 and 10004 were detected in the associated laboratory blank in concentrations exceeding those detected in the samples. One of the seven unknown SVOA compounds, tentatively identified as 4-methyl-2-pentanon-4-ol, was detected in the laboratory blank at an estimated concentration more than double the concentrations detected in the samples. The extremely low concentrations and detection of the compounds, at a higher concentration, in the laboratory blank, cast doubt on the validity of detection of these compounds in the sample. None of the other six unknown semi-volatile organics were detected in the laboratory blank. The source and nature of these compounds are unknown; however, due to their occurrence in extremely low concentrations, they are not considered cause for significant concern.

Total petroleum hydrocarbon (TPH) analysis for sample 10003 indicated the absence of any petroleum hydrocarbons in the sample.

\section{Sample 10005}

In addition to water from Water Well 8 , water from the sumps at the area 3 mud plant were used in the construction of ER-12-1. Water in this sump could have been derived from a number of wells on the NTS (Doug Duncan; DOE, personal communication). The origin of the water in the sump at the time of the construction of well ER-12-1 was unknown. Sample 10005 was collected for VOA, SVOA, tritium, gross alpha-beta-gamma spectroscopy, cations and anions analysis to determine the hydrochemical character of water from the area 3 mud plant sump. This sample was collected from the mud plant sump in area 3 at 0800 hours on August 12, 1991, and taken to the REECo laboratory in Mercury for analysis.

Radiological analysis of sample 10005 indicated an estimated tritium concentration of 237 $\mathrm{pCi} / \mathrm{l}$, which was slightly above the detection limit. Gross alpha radiation was reported to be $1.44 \mathrm{E}-8$ $\mu \mathrm{Ci} / \mathrm{ml}$ with a detection limit of $2.04 \mathrm{E}-9 \mu \mathrm{Ci} / \mathrm{ml}$. Gross beta radiation was reported to be $9.85 \mathrm{E}-9$ $\mu \mathrm{Ci} / \mathrm{ml}$ with a detection limit of $3.03 \mathrm{E}-9 \mu \mathrm{Ci} / \mathrm{ml}$. Gamma radiation was not detected in the sample. Radiological analysis of sample 10005 indicated all measured parameters to be well within the SNDW standards.

Volatile organic analysis of sample 10005 indicated the presence of methylene chloride at an estimated concentration of $1 \mu \mathrm{g} / \mathrm{l}$, and an unknown compound with a retention time of 1.05 minutes at an estimated concentration of $6 \mu \mathrm{g} / \mathrm{l}$. Neither of these compounds were detected in the associated laboratory blank analysis. The source of these compounds and the nature of the unknown compound are unknown, however, their presence at extremely low concentrations are not considered cause for significant concern. 
SVOA analysis of sample 10005 indicated the presence of 4-methyl-2-pentanon-4-ol at a concentration of $31 \mu \mathrm{g} / \mathrm{l}$. However, the detection of 4-methyl-2-pentanon-4-ol at a concentration of $51 \mu \mathrm{g} / \mathrm{l}$ in the laboratory blank cast considerable doubt on the validity of its detection in the sample. No other known semi-volatile organic compounds were detected.

Inorganic (metals and anions) analyses of sample 10005 were obtained by DRI's Water Analysis Laboratory in Reno, Nevada. All analyzed constituents (magnesium, sulfate, chloride, nitrate, arsenic, iron, manganese, copper, zinc, barium cadmium, chromium, lead, mercury, selenium, silver, and fluoride) were below the SNDW standard.

\section{Sample 10006}

Sample 10006 was collected at 2330 hours on August 27, 1991, from the sump at ER-12-1 to determine the hydrochemical characteristics of fluids recovered from the well during the drilling process. The well had not yet penetrated the static water level. Samples were collected for VOA, SVOA, tritium, gross alpha-beta-gamma spectroscopy, metals/anions, and PCB analysis. VOA, SVOA, PCB, and radiological analysis was performed by the REECo laboratory in Mercury. Inorganic analyses (metals/anions) were conducted by DRI's Water Analysis Laboratory in Reno.

Radiological analysis of sample 10006 indicated tritium concentration below background with a detection limit of $285 \mathrm{pCi} / 1$ for the method used. Gross alpha radiation was reported to be $1.58 \mathrm{E}-8$ $\mu \mathrm{Ci} / \mathrm{ml}$, below the detection limit of $1.78 \mathrm{E}-8 \mu \mathrm{Ci} / \mathrm{ml}$. Gross beta radiation was reported to be $3.00 \mathrm{E}-8$ $\mu \mathrm{Ci} / \mathrm{ml}$ with a detection limit of $2.26 \mathrm{E}-8 \mu \mathrm{Ci} / \mathrm{ml}$. Gamma radiation was not detected in the sample. Radiological analysis of sample 10006 indicated all measured parameters to be well within the SNDW standards.

Initial volatile organic analysis of sample 10006 indicated the presence of acetone and an unknown compound tentatively identified as 2-propanol in excess of the calibration range for the method used. The sample was re-run at a dilution factor of 5. A back-up sample (sample 10006b), collected at the same time, was also run at a dilution factor of 5. Sample 10006, run at a dilution factor of 5 , indicated the presence of acetone and methylene chloride at concentrations of 650 and $27 \mu \mathrm{g} / \mathrm{l}$, respectively. Sample $10006 \mathrm{~b}$, also run at a dilution factor of 5 , indicated the presence of acetone, methylene chloride and 4-methylene-2-pentanone at concentrations of 800,11 , and $8 \mu \mathrm{g} / \mathrm{l}$, respectively. Concentration values for methylene chloride and 4-methyl-2-pentanone in sample $10006 \mathrm{~b}$ are estimated. The unknown compound tentatively identified as 2-propanol in the original undiluted analysis of sample 10006 was not detected in either sample run at a dilution factor of 5 . In the associated laboratory blank, run at a dilution factor of 1 , methylene chloride was detected at a estimated concentration of $0.8 \mu \mathrm{g} / \mathrm{l}$. The detection of methylene chloride in the associated laboratory blank, although at a much lower estimated concentration, casts doubt on the validity of its detection in the samples. The acetone detected in both sample 10006 and the back-up sample $10006 \mathrm{~b}$ may be due to bottle contamination or the presence of the compound within fluids obtained from ER-12-1.

Semi-volatile organic analysis (SVOA) of sample 10006 indicated the presence of 2-chlorophenol, benzoic acid, 2-methylnaphthalene, acenaphthylene, di-n-butylphthalate, and bis 
(2-ethylhexyl)phthalate at estimated concentrations of $0.4,7,0.3,6,0.7$, and $2 \mu \mathrm{g} / \mathrm{l}$, respectively. Twenty-one unknown or tentatively identified SVOA compounds were also detected in sample 10006. The 16 unknown compounds had retention times within the gas chromatograph of 5.61,6.31, $14.45,18.26,21.94,24.12,26.66,28.13,29.05,29.45,30.4,31.61,32.8,33.54,33.75$, and 36.62 minutes, and estimated concentrations of $69,17,36,85,16,14,23,41,23,87,550,180,91,330$, 62 , and $36 \mu \mathrm{g} / \mathrm{l}$, respectively. The five tentatively identified SVOA compounds detected in sample 10006 include heptanoic acid, nonanoic acid, decanedioic acid, dodecanamide, and ethanol, with retention times of $14.22,18.14,22.43,23.16$, and 25.64 minutes, at estimated concentrations of 16 , $10,67,10$, and $29 \mu \mathrm{g} / \mathrm{l}$, respectively. The associated laboratory blank contained di-n-butylphthalate at an estimated concentration of $0.7 \mu \mathrm{g} / 1$, and three unknown compounds. The three unknown compounds detected in the laboratory blank had retention times of 5.6, 30.27, and 33.53 minutes, with estimated concentration of 94,17 , and $65 \mu \mathrm{g} / \mathrm{l}$, respectively. The source of these SVOA compounds (and nature of the unknown compounds) is not known.

The origin of the detected VOA and SVOA compounds are not known. It is known, however, that on August 14, 1996, two gallons of oil were put down the hole to lubricate the bit. This oil is a likely source of the VOA and SVOA compounds detected in this sample. This theory is further supported by analysis of levels of waste oil in sample 10009 (fluid being circulated in well ER-12-1) at concentrations of 40 to $47 \mathrm{mg} / \mathrm{Kg}$.

No PCBs were detected in sample 10006. Detection limits for the method used were $2.5 \mu \mathrm{g} / \mathrm{g}$ for oil, $0.866 \mu \mathrm{g} / \mathrm{g}$ for swipe, and $0.05 \mu \mathrm{g} / \mathrm{g}$ for soil.

Inorganic (metals and anions) analyses of sample 10006 were obtained by DRI's Water Analysis Laboratory in Reno, Nevada. All analyzed constituents (magnesium, sulfate, chloride, nitrate, arsenic, iron, manganese, copper, zinc, barium cadmium, chromium, lead, mercury, selenium, silver, and fluoride) were determined to be within SNDW standards.

\section{Sample 10007}

Sample 10007 was collected at 1900 hours on September 4, 1991, from 509.3 (1671 ft) below ground level following penetration of the static water level (swl at approximately $470 \mathrm{~m}(1542 \mathrm{ft}$ ) below ground level). The sample was collected by DRI using a discrete interval wireline sampler for safe drinking water analysis by the state of Nevada.

All analyzed inorganic constituents (magnesium, sulfate, chloride, nitrate, arsenic, iron, manganese, copper, zinc, barium cadmium, chromium, lead, mercury, selenium, silver, and fluoride) were determined to be within SNDW standards with the exception of chromium and lead. The SNDW standard for both chromium and lead is $0.05 \mathrm{mg} / \mathrm{l}$. Sample 10007 was determined to contain lead at a concentration of $0.3 \mathrm{mg} / \mathrm{l}$, and chromium at a concentration of $0.69 \mathrm{mg} / \mathrm{l}$. Subsequent sample analysis results and an inventory of the materials used in well construction indicated the most likely source for these elevated concentrations to be the plating used to protect the pipe threads on the drilling string. Repeated threading and unthreading of the drilling string may have resulted in abrasion and solution of the plating material, producing elevated chromium and lead concentrations in the fluid within the wellbore. 


\section{Sample 10008}

Samples 10008a and 10008b were collected at 1400 hours on September 27, 1991. These samples were collected in an effort to determine the source of the chromium detected in sample 10007. Sample 10008a consisted of drilling foam used in the well construction process at ER-12-1. Sample $10008 \mathrm{~b}$ was composed of drilling mud from the Baker tank at ER-12-1. Analysis by DRI's Water Analysis Laboratory in Reno indicated a chromium concentration in sample $10008 \mathrm{~b}$ of 0.01 $m \mathrm{~g} / \mathrm{l}$, and in sample $10008 \mathrm{a}$ of less than $0.01 \mathrm{mg} / \mathrm{l}$.

\section{Sample 10009}

Samples 10009a and 10009b were collected at 1240 and 1226 hours, respectively, on October 11,1991 . These samples were collected to determine the total petroleum hydrocarbon (TPH) content of the drilling fluid (60 to 120 vis mud; Table 5) prior to entering the well (sample 10009a) and immediately upon its return to the surface (sample 10009b). Sample 10009a was collected at the standpipe at the drilling rig; sample $10009 \mathrm{~b}$ was collected at the flowline where drilling fluids were discharged from the well. TPH analysis was conducted by REECo's Industrial Hygiene Laboratory. Gasoline and diesel fuel were not detected in either sample. Waste oil was detected in sample 10009a at a concentration of $47 \mathrm{mg} / \mathrm{kg}$ with a detection limit of $10 \mathrm{mg} / \mathrm{kg}$ for the method used. Sample $10009 \mathrm{~b}$ contained waste oil at a concentration of $40 \mathrm{mg} / \mathrm{kg}$ with a detection limit of $10 \mathrm{mg} / \mathrm{kg}$.

\section{Sample 10010}

This sample was collected for safe drinking water analysis prior to discharging pumped fluid from the well to land surface. Approximately 578,460 liters (152,630 gallons) of fluid were produced from the well by pumping and developing prior to sampling. Samples were collected for cations, anions, nitrate, gross alpha, radium-226 and -228 , tritium, and strontium- 90 activity. Sample 10010 was collected at 1215 hours on March 15, 1991, and taken to Lockheed Analytical Laboratory in Las Vegas, Nevada, for analysis.

Radiological analysis of sample 10010 indicated a tritium activity of $167 \mathrm{pCi} / \mathrm{l}$ with an estimated uncertainty of $51 \mathrm{pCi} / 1$ and a detection limit of $66 \mathrm{pCi} / 1$ (based upon a counting period of 300 minutes on a Quantulus Liquid scintillation counter; Russ Stimmel, LES Laboratory, formerly Lockheed Analytical Laboratory; personal communication, January 6, 1997). Gross alpha activity was reported to be $21 \mathrm{pCi} / \mathrm{l}$ with an estimated uncertainty of $8 \mathrm{pCi} / 1$ and a detection limit of $8 \mathrm{pCi} / \mathrm{l}$. Strontium- 90 activity was reported to be $0.93 \mathrm{pCi} / \mathrm{l}$ with an estimated uncertainty of $0.62 \mathrm{pCi} / 1$ and a detection limit of $1.1 \mathrm{pCi} / 1$. Radium-226 activity was reported to be $2.0 \mathrm{pCi} / 1$ with an estimated uncertainty of $0.19 \mathrm{pCi} / \mathrm{l}$ and a detection limit of $0.04 \mathrm{pCi} / \mathrm{l}$. Radium-228 activity was reported to be $2.6 \mathrm{pCi} / \mathrm{l}$ with an estimated uncertainty of $0.8 \mathrm{pCi} / \mathrm{l}$ and a detection limit of $1.1 \mathrm{pCi} / 1$.

All analyzed constituents (magnesium, sulfate, chloride, nitrate, arsenic, iron, manganese, copper, zinc, barium cadmium, chromium, lead, mercury, selenium, silver, and fluoride) were determined to be within SNDW standards. 
Sample 10011

Sample 10011 was collected at the completion of initial aquifer testing at ER-12-1. The sample was collected at 1240 hours on April 1, 1992 and taken to Lockheed Analytical Laboratory in Las Vegas, Nevada for analysis. Analysis was requested for compliance with applicable SDWA standards (cations, anions, nitrate, and gross alpha-beta-gamma spectroscopy). At the time the sample was collected, approximately 648,600 liters (171,200 gallons) of fluid had been recovered from the well during development and testing activities.

Radiological analysis of sample 10011 reported gross alpha activity to be $22 \mathrm{pCi} / 1$ with an estimated uncertainty of $9 \mathrm{pCi} / 1$ and a detection limit of $11 \mathrm{pCi} / 1$. Gross beta activity was reported to be $17 \mathrm{pCi} / \mathrm{l}$ with an estimated uncertainty of $9 \mathrm{pCi} / 1$ and a detection limit of $13 \mathrm{pCi} / \mathrm{l}$. Gamma spectrum analysis reported a total activity of $231 \mathrm{pCi} / \mathrm{l}$. The level of gamma activity observed in the sample was due primarily to high concentrations of lead-214 and bismuth-214. Both lead-214 and bismuth-214 were detected in the sample at concentrations slightly in excess of $100 \mathrm{pCi} / \mathrm{l}$. Lockheed Analytical Laboratory, in their analysis summary, indicated the high concentration of lead-214 and bismuth-214 were probably due to the presence of radon-222 and its daughters. Since the sample was counted two days after collection, much of the radon-222 and its daughters would still have been present in the sample.

All analyzed constituents (magnesium, sulfate, chloride, nitrate, arsenic, iron, manganese, copper, zinc, barium cadmium, chromium, lead, mercury, selenium, silver, and fluoride) were determined to be within SNDW standards with the exception of manganese and iron. Manganese and iron concentrations within sample 10011 were reported at 0.21 and $1.1 \mathrm{mg} / \mathrm{l}$, respectively. SNDW standards set limits of 0.1 for manganese and $0.6 \mu \mathrm{g} / \mathrm{l}$ for iron. The source of the elevated manganese and iron concentrations in sample 10011 is unknown. Sample 10011 was collected immediately prior to the conclusion of pumping during the long-term aquifer test portion of initial aquifer testing at ER-12-1. At that time, approximately 648,600 liters (171,200 gallons) of fluid had been recovered from the well. Sample 10010, collected at the end of well development, after approximately 578,460 liters ( 152,630 gallons) of fluid had been recovered from the well, contained manganese and iron concentrations well below the SNDW standards. A possible explanation is that by the end of the long-term aquifer test, draw-down within the well was sufficient to allow intervals in the well, which did not normally contribute to discharge from the well, to begin yielding fluids. These fluids would consist, to a large degree, of drilling and development fluids from zones that may not have been purged until this time.

\section{Geochemical Characterization Sample}

\section{Sample Number 10012}

Sample 10012 was acquired on January 5, 1993, at the end of the long-term aquifer test conducted on the upper zone 518 to $554 \mathrm{~m}$ (1700 to $1820 \mathrm{ft}$ ). The purpose of this sample was to acquire the ionic and isotopic chemistry of the water and use it to determine the source of the fluid within this zone. Samples were acquired for volatile and semivolatile organic analyses, total 
petroleum hydrocarbons, ionic chemistry, trace metals, stable and radiogenic isotopes, and total organic carbon. The results of these analyses are presented in Table 39.

Several volatile organic compounds were detected in sample 10012 above the detection limit and were not detected in associated blanks. These consist of chloroform $(5.0 \mu \mathrm{g} / \mathrm{l})$ bromodichloromethane $(7.0 \mu \mathrm{g} / \mathrm{l})$, dibromochloromethane $(16 \mu \mathrm{g} / 1)$, bromoform $(65 \mu \mathrm{g} / \mathrm{l})$, and an unknown compound with a retention time of 1.54 minutes $(17 \mu \mathrm{g} / 1)$. Two semivoloatile organic compounds were identified in the well water. These consisted of an unknown compound with a retention time of 5.75 minutes with an estimated concentration of $9 \mu \mathrm{g} / \mathrm{l}$ and a second compound, tentatively identified as Phenol, 4,4'-(1-methylethyli), with a concentration of $10 \mu \mathrm{g} / 1$. No petroleum hydrocarbons were detected in the water.

The ionic chemistry of sample 10012, and that of samples previously taken from carbonate wells, volcanic wells, and wells completed in the Eleana, are presented in Figure 48. The results indicate that sample 10012 had anomalous calcium, chloride, magnesium, and sulfate values concentrations relative to similar wells on the NTS. These elevated concentrations were also detected in sample 10007 and 10010. Examination of the bromide concentration in sample 10012 indicates that drilling fluids compose approximately 20 to 25 percent of the fluid in this sample. Elevated concentrations of calcium and chloride may be due to calcium chloride used in the well as a cement accelerator. Elevated levels of sulfate may be due to the use of bentonite mud in the borehole. In either case, it is highly probable that analysis of sample 10012 is not representative of formation water.

Results of the radiochemical analysis from LLNL are also presented in Table 39. Portions of the following discussion are excerpted from LLNL (1993). All radionuclides measured in the ground water from ER-12-1 were found to be at or below detection limits, with the exception of tritium. Tritium was measured at $361 \mathrm{pCi} / \mathrm{L}(113 \mathrm{TU})$ or approximately 50 times less than the drinking water standard $(20,000 \mathrm{pCi} / \mathrm{L})$. The source of the tritium was speculated to have originated from the drilling fluids (LLNL, 1993).

It is estimated that a total of $8.4 \times 10^{6}$ liters $\left(2.2 \times 10^{6}\right.$ gallons $)$ of drilling and development fluid were circulated through well ER-12-1. Approximately $6.2 \times 10^{6}$ liters ( $1.6 \times 10^{6}$ gallons) were trucked away from the well and $0.6 \times 10^{6}$ liters $\left(0.16 \times 10^{6}\right.$ gallons $)$ were discharged to land surface and $0.32 \times 10^{6}$ liters $\left(0.084 \times 10^{6}\right.$ gallons) were left in the sump at ER-12-1. These calculations result in approximately $1.2 \times 10^{6}$ liters $\left(0.32 \times 10^{6}\right.$ gallons $)$ of drilling fluid remaining in well ER-12-1. This quantity of fluid has been partially purged by the approximately $1.2 \times 10^{6}$ liters $(0.32$ $\times 10^{6}$ gallons) that drained from the upper zone into the three lower zones (during the period of April 24 to October 23, 1992) while the borehole remained open. Sample 10005 (fluid from the mud plant sump) had a tritium activity of $237 \mathrm{pCi} / \mathrm{L}$. It is highly probable, assuming there was some dilution of the drilling fluid with the formation water, that some fraction of the drilling fluid remained in the upper zone in well ER-12-1 (as indicated by the elevated levels of bromide in the sample; $4.9 \mathrm{mg} / \mathrm{L}$ ), and potentially contributed to a portion of the tritium activity detected in sample 10012 . The maximum activity, based on the aforementioned data, that could be potentially contributed by 
Table 39. Geochemical Results from the Upper Zone, 518 to $555 \mathrm{~m}$ (1700 to $1820 \mathrm{ft}$ ), of Well ER-12-1

\begin{tabular}{|c|c|c|c|}
\hline \multicolumn{4}{|c|}{ Volatile Organic Analysis } \\
\hline Compound & $\begin{array}{c}\text { Concentration Units } \\
\mu \mathrm{g} / \mathrm{l}\end{array}$ & Qualifier & \\
\hline Chloromethane & 2.0 & $\mathrm{U}$ & \\
\hline Bromomethane & 2.0 & $\mathrm{U}$ & \\
\hline Vinyl Chloride & 2.0 & $\mathrm{U}$ & \\
\hline Chloroethane & 2.0 & $\mathrm{U}$ & \\
\hline Methylene Chloride & 1.0 & $\mathrm{U}$ & \\
\hline Acetone & 1.0 & BJ & \\
\hline Carbon Disulfide & 1.0 & $\mathrm{U}$ & \\
\hline 1,1-Dichloroethene & 1.0 & $\mathrm{U}$ & \\
\hline 1,1-Dichloroethane & 1.0 & $\mathrm{U}$ & \\
\hline 1,2-Dichloroethane (total) & 1.0 & $\mathrm{U}$ & \\
\hline Chloroform & 5.0 & & \\
\hline 1,2-Dichloroethane & 1.0 & U & \\
\hline 2-Butanone & 2.0 & $\mathrm{U}$ & \\
\hline 1,1,1-Trichloroethane & 1.0 & $\mathrm{U}$ & \\
\hline Carbon Tetrachloride & 1.0 & $\mathrm{U}$ & \\
\hline Vinyl Acetate & 2.0 & U & \\
\hline Bromodichloromethane & 7.0 & & \\
\hline 1,2-Dichloropropane & 1.0 & $\mathrm{U}$ & \\
\hline cis-1,3-Dichloropropene & 1.0 & $\mathrm{U}$ & \\
\hline Trichloroethene & 1.0 & $\mathrm{U}$ & \\
\hline Dibromochloromethane & 16. & & \\
\hline 11,1,2-Trichloroethane & 1.0 & $\mathrm{U}$ & \\
\hline Benzene & 1.0 & $\mathrm{U}$ & \\
\hline trans-1,3-Dichloropropene & 1.0 & $\mathrm{U}$ & \\
\hline Bromoform & 65. & $\mathrm{E}$ & \\
\hline 4-Methyl-2-Pentanone & 2.0 & $\mathrm{U}$ & \\
\hline 2-Hexanone & 2.0 & $U$ & \\
\hline Tetrachloroethehe & 1.0 & $\mathrm{U}$ & \\
\hline 1,1,2,2-Tetrachloroethane & 1.0 & $\mathrm{U}$ & \\
\hline Toluene & 1.0 & $\mathrm{U}$ & \\
\hline Chlorobenzene & 1.0 & $\mathrm{U}$ & \\
\hline Ethylbenzene & 1.0 & $\mathrm{U}$ & \\
\hline Styrene & 1.0 & U & \\
\hline Xylene (total) & 1.0 & $\mathrm{U}$ & \\
\hline Compound & Return Time & Est. Conc. & Qualifier \\
\hline Unknown & 1.54 & 17.00 & $\mathrm{~J}$ \\
\hline
\end{tabular}


Table 39. Geochemical Results from the Upper Zone, 518 to $555 \mathrm{~m}$ (1700 to $1820 \mathrm{ft}$ ), of Well ER-12-1 (continued).

Semivolatile Organics Analysis

\begin{tabular}{|c|c|c|}
\hline Compound & $\begin{array}{c}\text { Concentration Units } \\
\mu \mathrm{g} / \mathrm{l}\end{array}$ & Qualifier \\
\hline Phenol & 10 & $\mathrm{U}$ \\
\hline bis (2-Chloroethyl) ether & 10 & $\mathrm{U}$ \\
\hline 2-Chlorophenol & 10 & $\mathrm{U}$ \\
\hline 1,3-Dichlorobenzene & 10 & $\mathrm{U}$ \\
\hline 1,4-Dichlorobenzene & 10 & $\mathrm{U}$ \\
\hline Benzyl alcohol & 10 & $\mathrm{U}$ \\
\hline 1,2-Dichlorobenzene & 10 & $\mathrm{U}$ \\
\hline 2-Methylphenol & 10 & $\mathrm{U}$ \\
\hline bis (2-chlorolisopropyl) ether & 10 & U \\
\hline 4-Methylphenol & 10 & $\mathrm{U}$ \\
\hline N-Nitroso-Di-n-propylamine & 10 & $\mathrm{U}$ \\
\hline Hexachloroethane & 10 & $\mathrm{U}$ \\
\hline Nitrobenzene & 10 & $\mathrm{U}$ \\
\hline Isophorone & 10 & $\mathrm{U}$ \\
\hline 2-Nitrophenol & 10 & $\mathrm{U}$ \\
\hline 2,4-Dimethylphenol & 10 & $\mathrm{U}$ \\
\hline Benzoic acid & 50 & $\mathrm{U}$ \\
\hline bis (2-Chloroethoxy) methane & 10 & $\mathrm{U}$ \\
\hline 2,4-Dichlorophenol & 10 & $\mathrm{U}$ \\
\hline 1,2,4-Trichlorobenzene & 10 & $\mathrm{U}$ \\
\hline Naphthalene & 10 & $\mathrm{U}$ \\
\hline 4-Chloroaniline & 10 & $\mathrm{U}$ \\
\hline Hexachlorobutadiene & 10 & $\mathrm{U}$ \\
\hline 4-Chloro-3-methylphenol & 10 & $\mathrm{U}$ \\
\hline 2-Methylnaphthalene & 10 & $\mathrm{U}$ \\
\hline Hexachlorocyclopentadiene & 10 & $\mathrm{U}$ \\
\hline 2,4,6-Trichlorophenol & 10 & $\mathrm{U}$ \\
\hline $2,4,5$-Trichlorophenol & 50 & $\mathrm{U}$ \\
\hline 2-Chloronaphthalene & 10 & $\mathrm{U}$ \\
\hline 2-Nitroaniline & 50 & $\mathrm{U}$ \\
\hline Dimethylphthalate & 10 & $\mathrm{U}$ \\
\hline Acenaphthylene & 10 & U \\
\hline 2,6-Dinitrotoluene & 10 & $\mathrm{U}$ \\
\hline 3-Nitroaniline & 50 & $\mathrm{U}$ \\
\hline Acenaphthene & 10 & $\mathrm{U}$ \\
\hline 2,4-Dinitrophenol & 50 & $\mathrm{U}$ \\
\hline 4-Nitrophenol & 50 & $\mathrm{U}$ \\
\hline Dibenzofuran & 10 & $\mathrm{U}$ \\
\hline
\end{tabular}


Table 39. Geochemical Results from the Upper Zone, 518 to $555 \mathrm{~m}$ (1700 to $1820 \mathrm{ft}$ ), of Well ER-12-1 (continued).

Semivolatile Organic Analysis

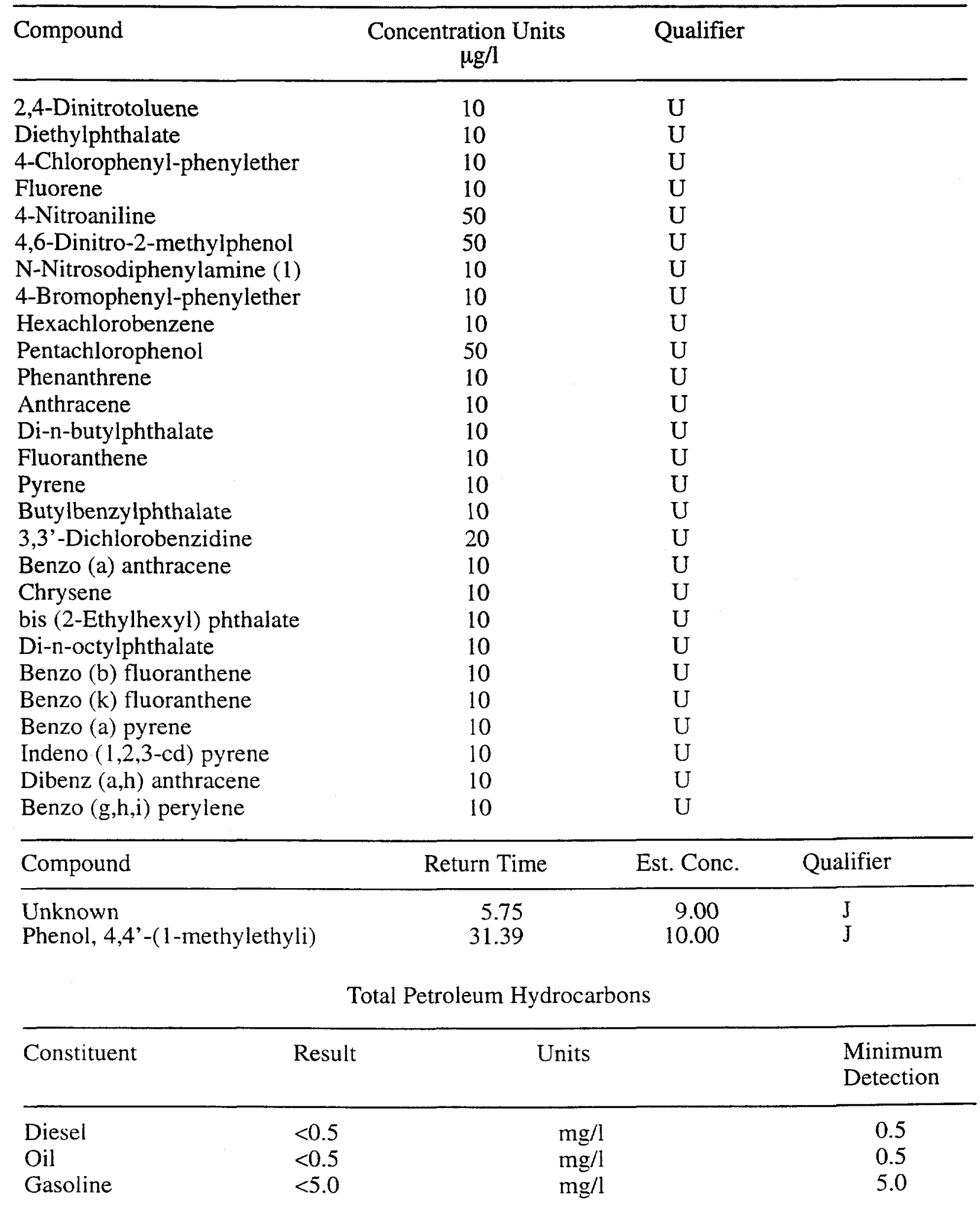


Table 39. Geochemical Results from the Upper Zone, 518 to $555 \mathrm{~m}$ (1700 to $1820 \mathrm{ft}$ ), of Well ER-12-1 (continued).

Resuits of Ionic Analyses

\begin{tabular}{|c|c|}
\hline Constituent & $\mathrm{mg} / \mathrm{l}$ \\
\hline $\mathrm{HCO} 3$ & 319 \\
\hline $\mathrm{Cl}$ & 46.7 \\
\hline SO4 & 357 \\
\hline NO3 & 1.64 \\
\hline $\mathrm{Na}$ & 74.2 \\
\hline $\mathrm{K}$ & 5.10 \\
\hline $\mathrm{Ca}$ & 96.8 \\
\hline $\mathrm{Mg}$ & 64.8 \\
\hline $\mathrm{NO} 3$ as $\mathrm{N}$ & 0.37 \\
\hline TDS & 876 \\
\hline $\mathrm{Br}$ & 4.9 \\
\hline $\mathrm{F}$ & 0.33 \\
\hline TKN & $<0.1$ \\
\hline $\mathrm{NH} 4$ & .01 \\
\hline $\mathrm{NO} 2$ & 0.02 \\
\hline TPO4 & 8.59 \\
\hline $\mathrm{Al}$ & $<0.02$ \\
\hline $\mathrm{Sb}$ & 0.026 \\
\hline As & 0.48 \\
\hline $\mathrm{Ba}$ & 0.011 \\
\hline $\mathrm{Be}$ & $<0.001$ \\
\hline $\mathrm{Cd}$ & $<0.001$ \\
\hline $\mathrm{Cr}$ & $<0.01$ \\
\hline $\mathrm{Co}$ & $<0.01$ \\
\hline $\mathrm{Cu}$ & 0.008 \\
\hline $\mathrm{Fe}$ & 0.51 \\
\hline $\mathrm{Pb}$ & 0.017 \\
\hline $\mathrm{Li}$ & 1.37 \\
\hline $\mathrm{Mn}$ & 0.05 \\
\hline $\mathrm{Hg}$ & $<0.0002$ \\
\hline $\mathrm{Ni}$ & $<0.01$ \\
\hline $\mathrm{Se}$ & 0.006 \\
\hline $\mathrm{Ag}$ & $<0.005$ \\
\hline $\mathrm{TI}$ & $<0.001$ \\
\hline V & $<0.02$ \\
\hline $\mathrm{Zn}$ & $<0.005$ \\
\hline TOC & $<0.1$ \\
\hline $\mathrm{Fe} 2$ & $<0.05$ \\
\hline $\mathrm{Fe} 3$ & 0.51 \\
\hline
\end{tabular}


Table 39. Geochemical Results from the Upper Zone, 518 to $555 \mathrm{~m}$ (1700 to $1820 \mathrm{ft}$ ), of Well ER-12-1 (continued).

Stable Isotopes

\begin{tabular}{lll}
\hline Constituent & Per Mil & Standard \\
\hline$\delta^{18} \mathrm{O}$ & -12.4 & \\
$\delta \mathrm{D}$ & -94 & VSMOW \\
$\delta^{13} \mathrm{C}$ & -10.0 & VSMOW \\
\end{tabular}

\begin{tabular}{|c|c|c|c|c|}
\hline \multicolumn{5}{|c|}{ Radioisotopes } \\
\hline Constituent & Activity & $\begin{array}{l}\text { Estimated } \\
\text { Uncertainty }\end{array}$ & MDA & Units \\
\hline Radon-222 & 403 & 17. & 2.3 & $\mathrm{pCi} / \mathrm{L}$ \\
\hline Constituent & Activity & $\begin{array}{l}\text { Estimated } \\
\text { Uncertainty }\end{array}$ & \multicolumn{2}{|l|}{ Units } \\
\hline Gr-Alpha & 4.95 E-09 & $30.3 \%$ & \multicolumn{2}{|l|}{$\mu \mathrm{Ci} / \mathrm{ml}$} \\
\hline Gr-Beta & $2.40 \mathrm{E}-09$ & $33.3 \%$ & \multicolumn{2}{|l|}{$\mu \mathrm{Ci} / \mathrm{ml}$} \\
\hline Gamma & $\begin{array}{l}\text { no radionuclide } \\
\text { detected }\end{array}$ & N/A & \multicolumn{2}{|c|}{$\mathrm{N} / \mathrm{A}$} \\
\hline Constituent & Activity & $\begin{array}{l}\text { Estimated } \\
\text { Uncertainty }\end{array}$ & \multicolumn{2}{|l|}{ Units } \\
\hline Carbon-14 & 10.9 & 1.0 & \multirow{3}{*}{\multicolumn{2}{|c|}{$\begin{array}{l}\text { Percent activity at } \\
1950 \mathrm{C}-14 \text { activity } \\
\text { Years } \mathrm{BP}\left({ }^{13} \mathrm{C} \text { corrected }\right) \\
\text { Note } \delta^{13} \mathrm{C}_{\mathrm{pDB}}=12.2 \text { per mil } \\
\mathrm{pCi} / \mathrm{L}\end{array}$}} \\
\hline & 17,800 & 665 & & \\
\hline Tritium (DRI) & 311 & 11 & & \\
\hline Constituent & Activity & $\begin{array}{l}\text { Estimated } \\
\text { Uncertainty }\end{array}$ & MDA & Units \\
\hline Radium-226 & 0.28 & 0.37 & 0.62 & $\mathrm{pCi} / 1$ \\
\hline Radium-228 & 1.91 & 0.79 & 1.5 & $\mathrm{pCi} / \mathrm{l}$ \\
\hline Uranium & 2.042 & 0.051 & 0.10 & $\mu \mathrm{g} / \mathrm{l}$ \\
\hline
\end{tabular}


Table 39. Geochemical Results from the Upper Zone, 518 to $555 \mathrm{~m}$ (1700 to $1820 \mathrm{ft}$ ), of Well ER-12-1 (continued).

LLNL Radiochemistry

\begin{tabular}{|c|c|c|c|c|}
\hline \multicolumn{5}{|c|}{ Radioisotopes } \\
\hline Constituent & Activity & $\begin{array}{l}\text { Estimated } \\
\text { Uncertainty }\end{array}$ & MDA & Units \\
\hline${ }^{3} \mathrm{H}-{ }^{3} \mathrm{He}$ & 361 & 34. & 6.4 & $\mathrm{pCi} / \mathrm{L}$ \\
\hline${ }^{60} \mathrm{Co}$ & $\leq 0.27$ & & 0.27 & $\mathrm{pCi} / \mathrm{L}$ \\
\hline${ }^{85} \mathrm{Kr}$ & $\leq 30$ & & 30 & $\mathrm{pCi} / \mathrm{L}$ \\
\hline${ }^{90} \mathrm{Sr}$ & $\leq 0.5$ & & 0.5 & $\mathrm{pCi} / \mathrm{L}$ \\
\hline${ }^{99} \mathrm{Tc}$ & $\leq 4.5$ & & 4.5 & $\mathrm{pCi} / \mathrm{L}$ \\
\hline${ }^{125} \mathrm{Sb}$ & $\leq 0.74$ & & 0.74 & $\mathrm{pCi} / \mathrm{L}$ \\
\hline${ }^{137} \mathrm{Cs}$ & $\leq 0.28$ & & 0.28 & $\mathrm{pCi} / \mathrm{L}$ \\
\hline${ }^{234} \mathrm{U}$ & 7.93 & & & $\mathrm{pCi} / \mathrm{L}$ \\
\hline${ }^{235} \mathrm{U}$ & 0.073 & & & $\mathrm{pCi} / \mathrm{L}$ \\
\hline${ }^{238} \mathrm{U}$ & 1.59 & & & $\mathrm{pCi} / \mathrm{L}$ \\
\hline${ }^{36} \mathrm{Cl}$ & 0.0071 & & & $\mathrm{pCi} / \mathrm{L}$ \\
\hline${ }^{14} \mathrm{C}$ & 0.1069 & & & pmc \\
\hline${ }^{13} \mathrm{C}$ & -9.2 & & & per mil \\
\hline${ }^{36} \mathrm{Cl} / \mathrm{Cl}$ & \multicolumn{3}{|c|}{$5.06 \times 10^{-12} \pm 5.9 \times 10^{-14}$} & \\
\hline${ }^{36} \mathrm{Cl}$ & \multicolumn{2}{|l|}{$360 \times 10^{7}$} & & atoms $/ \mathrm{L}$ \\
\hline${ }^{87} \mathrm{Sr} /{ }^{86} \mathrm{Sr}$ & \multicolumn{2}{|c|}{$0.71175 \pm 0.00002$} & & \\
\hline$\delta^{87} \mathrm{Sr}$ & \multicolumn{2}{|c|}{3.60} & & \\
\hline${ }^{234} \mathrm{U} /{ }^{238} \mathrm{U}$ & \multicolumn{2}{|c|}{$0.000275 \pm 0.0000003$} & & \\
\hline${ }^{234} \mathrm{U} / 235 \mathrm{U}$ & \multicolumn{2}{|c|}{0.03794} & & \\
\hline${ }^{234} \mathrm{U} /{ }^{238} \mathrm{U}$ & \multicolumn{2}{|l|}{4.99} & & activity ratio \\
\hline${ }^{4} \mathrm{He}$ & $4.89 \times 10^{12}$ & $2 \%$ & & atoms $/ \mathrm{ml}$ \\
\hline${ }^{20} \mathrm{Ne}$ & $2.55 \times 10^{13}$ & $2 \%$ & & atoms $/ \mathrm{ml}$ \\
\hline${ }^{36} \mathrm{Ar}$ & $8.78 \times 10^{13}$ & $2 \%$ & & atoms $/ \mathrm{ml}$ \\
\hline${ }^{82} \mathrm{Kr}$ & $5.17 \times 10^{11}$ & $2 \%$ & & atoms $/ \mathrm{ml}$ \\
\hline${ }^{129} \mathrm{Xe}$ & $1.36 \times 10^{11}$ & $2 \%$ & & atoms/ml \\
\hline${ }^{3} \mathrm{He}$ & $7.38 \times 10^{6}$ & $2 \%$ & & atoms $/ \mathrm{ml}$ \\
\hline
\end{tabular}


Milliequivalents/L
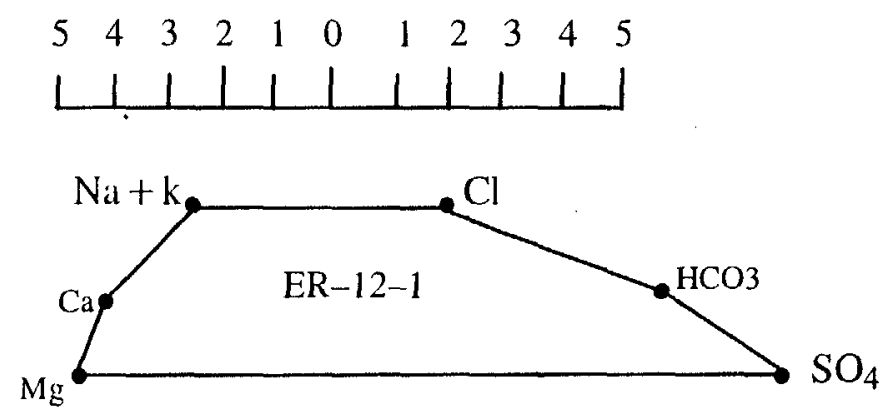

$\bar{s}$
Millequivalents/L
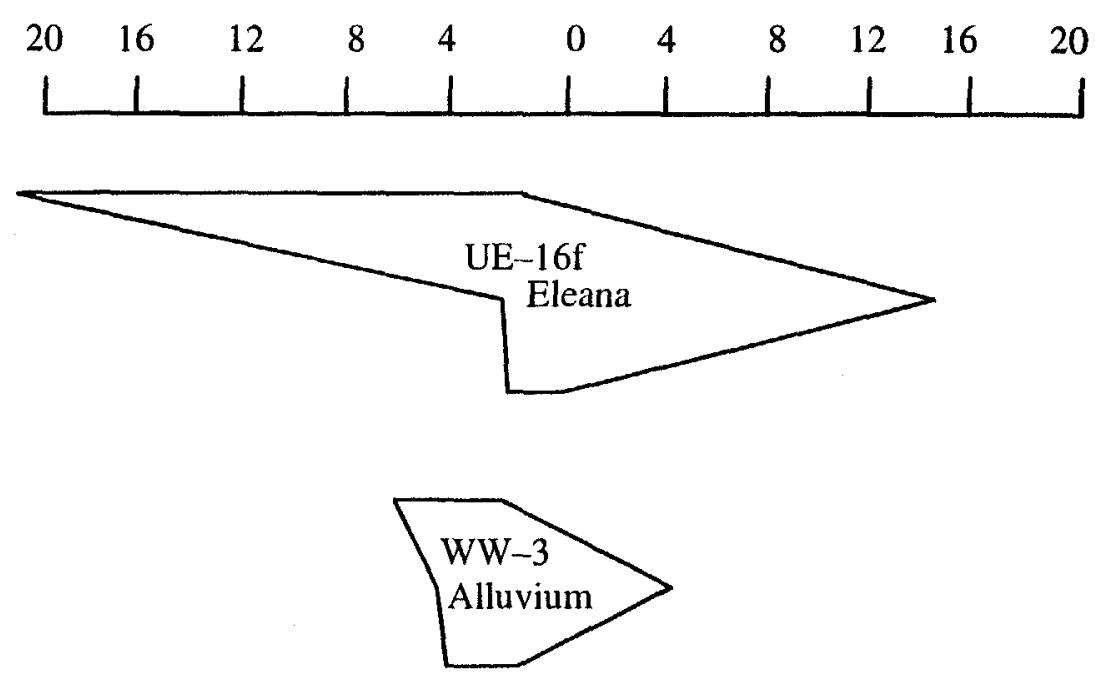

Eleana

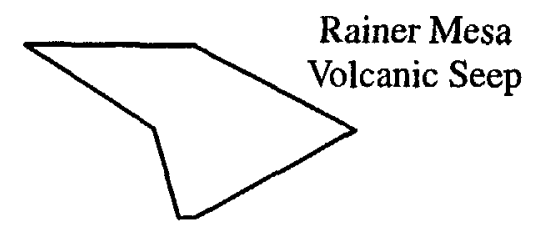

Figure 48. Stiff diagrams for carbonate, Eleana, and volcanic wells. 
drilling fluid to sample 10012 is estimated to be $60 \mathrm{pCi} / \mathrm{L}$. An alternative hypothesis is the source of the tritium found in well ER-12-1 (which is 1.5 times greater than the tritium activity found in sample 10005) was the U12e infiltration ponds located $460 \mathrm{~m}$ (1500 ft) to the east of well ER-12-1. These sumps have been receiving tritiated effluent with activities of $2.4 \times 10^{6} \mathrm{pCi} / \mathrm{L}$ for over two decades (Russell et al., 1993). The sumps are composed of a thin veneer of alluvium overlying fractured dolomite. A third potential source for the tritium found in the upper zone of ER-12-1 is the underground nuclear tests conducted in Rainier Mesa, located approximately $2.1 \mathrm{~km}$ ( 1.3 miles) to the east of the well site. These detonations, in addition to being more distant than the tritium ponds, were conducted in zeolitized tuffs, which have some of the lowest hydraulic conductivities measured on the NTS (Thordarson, 1965). The underground tests are a less likely source of tritium, owing to their greater distance and an expected lower permeability of the surrounding geologic matrix, relative to the tunnel ponds. The existing data, however, do not differentiate or negate any of the aforementioned hypotheses. Time series sampling, at a pump rate of $190 \mathrm{l} / \mathrm{min}(50 \mathrm{gpm})$, in the upper zone is recommended if the source of tritium in ER-12-1 needs to be resolved. If the source of tritium was drilling fluids, the concentrations will dilute with additional pumping and decrease. If the tritium was from the ponds or from underground nuclear tests, then pumping will cause further migration of the tritium to the well, increasing concentrations.

The ${ }^{14} \mathrm{C}$ content of the groundwater from ER-12-1 was much lower than natural atmospheric ${ }^{14} \mathrm{C}$ and indicated that the groundwater had an apparent age of 18,500 years. The $\delta^{13} \mathrm{C}$ signature of the groundwater $(-9.2$ per mil) reflected natural values and was indicative of dissolved carbon derived from the unsaturated zone. This suggested an insignificant amount of Paleozoic carbonate $\left(\delta^{13} \mathrm{C} \sim 0\right.$ per mil) dissolution had occurred. This conclusion was highly uncertain due to the probable influence of the water added down-hole during drilling. Therefore, the low ${ }^{14} \mathrm{C}$ content of the water sampled at ER-12-1 may have been derived from the naturally occurring groundwater in the aquifer, or a mixture of drilling fluids and the aquifer water (LLNL, 1993).

The ${ }^{36} \mathrm{Cl} / \mathrm{Cl}$ ratio measurement for groundwater from ER-12-1 had an environmental level, but was an order of magnitude higher than naturally occurring groundwater at the NTS from similar depths. This suggested that an anthropogenic chloride component had been introduced into the water, which was consistent with the higher than normal chloride concentration $(46.7 \mathrm{mg} / \mathrm{L})$. The drilling fluids, $\mathrm{Li} / \mathrm{Br}$, or the cement accelerators were likely sources (LLNL, 1993).

Dissolved noble gas measurements in the groundwater sampled from ER-12-1 indicated that a component of modern injected air was introduced in the groundwater. This was best seen in the abundance of ${ }^{20} \mathrm{Ne}$, which was a factor of three to six higher than had been found in other UGTA well waters to date. The ${ }^{20} \mathrm{Ne}$ abundance in modern air is high relative to the other dissolved noble gases. It is probable that the noble gas pattern in ER-12-1 was disturbed by atmospheric introduction during well development (LLNL, 1993).

The ${ }^{87} \mathrm{Sr} /{ }^{86} \mathrm{Sr}$ ratio $(0.7118)$ in the groundwater from ER-12-1 was at a natural level. The ${ }^{235} \mathrm{U}$ concentration was also at an environmental level, as was the ${ }^{234} \mathrm{U} /{ }^{238} \mathrm{U}$ ratio, which had an activity ratio greater than one, suggesting some natural disequilibria between the solubility of the two 
isotopes in the crystal lattice structure of the aquifer matrix. Uncertainties in the effects of well development render inconclusive the interpretations based on the strontium and uranium isotopes (LLNL, 1993).

The stable isotopic signature of sample 10012 is plotted in Figure 49. This plot also contains representative isotopic signatures of Rainier Mesa seeps and wells on the NTS completed in various formations. The data for these other wells are presented in Table 40. The plot indicates that the water sampled from the upper zone of well ER-12-1 was intermediate isotopically to the water found in seeps in Rainier Mesa and water from Well 8. If the water from ER-12-1 was contaminated 20 to 25 percent with isotopically depleted water from Water Well 8 and Wells $\mathrm{C}$ and C-1 (which feed the sump at the Area 3 mud plant), then the true signature was even more enriched than that shown in Figure 48. The one conclusion that can be drawn from this plot is formation water in Well ER-12-1 was isotopically similar to water that originated as precipitation on Rainier Mesa.

\section{SUMMARY}

The objective of well ER-12-1, N886,640.26 E640,538.85 Nevada Central Coordinates, was to determine the hydrogeology of Paleozoic carbonate rocks and of the Eleana Formation, a regional aquitard, in an area potentially down-gradient from underground nuclear testing conducted in nearby Rainier Mesa. Drilling of the $1094 \mathrm{~m}$ (3588 ft) well began on July 19, 1991, and reached TD on October 17, 1991. Drilling problems included hole deviation and hole instability that prevented the timely completion of this borehole. Drilling methods used include rotary tri-cone and rotary hammer drilling with conventional and reverse circulation using air/water, air/foam (Davis mix), and bentonite mud.

Geologic cuttings and geophysical logs were obtained from the well. The rocks penetrated by the ER-12-1 drillhole are a complex assemblage of Ordovician, Silurian, Devonian, and Mississippian sedimentary rocks that are bounded by numerous faults that show substantial stratigraphic offset. The final $7.3 \mathrm{~m}(24 \mathrm{ft})$ of this hole penetrated an unusual intrusive rock of Cretaceous age. The geology of this borehole was substantially different from that expected, with the Tongue Wash Fault encountered at a much shallower depth, Paleozoic rocks shuffled out of stratigraphic sequence, and the presence of an altered biotite-rich lamprophyre rock at the bottom of the borehole.

Conodont $\mathrm{CAI}$ analyses and rock pyrolysis analyses indicate that the carbonate rocks in ER-12-1, as well as the intervening sheets of Eleana siltstone, have been thermally overprinted following movement on the faults that separate them. The probable source of heat for this thermal disturbance is the lamprophyre intrusion encountered at the bottom of the hole, and its age establishes that the major fault activity must have occurred prior to $102.3+0.5 \mathrm{Ma}$ (middle Cretaceous).

Geophysical logs run in the saturated and unsaturated sections of the borehole were invaluable for interpretation of stratigraphy and structure. Problems encountered during logging were lack of 


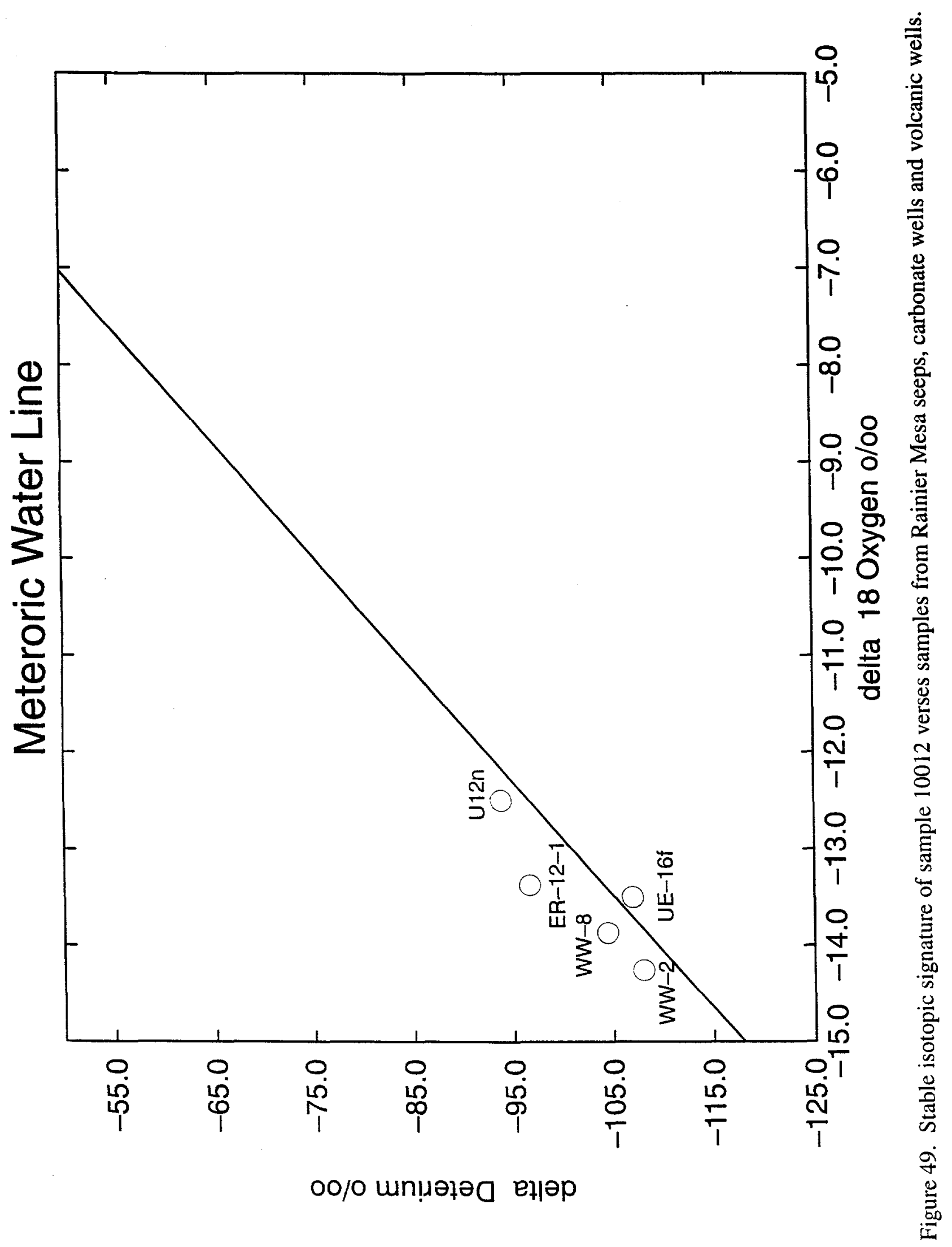


Table 40. Stable Isotopic Signature of Wells and Seeps on the NTS.

\begin{tabular}{|c|c|c|}
\hline Well & $\delta^{18} \mathrm{O}$ & $\delta \mathrm{D}$ \\
\hline \multicolumn{3}{|l|}{ Carbonate Wells } \\
\hline UE1q & -14.6 & -109 \\
\hline UE-1b & -13.8 & -105 \\
\hline Well C-1 & -14.4 & -109 \\
\hline Well C & -14.4 & -109 \\
\hline Test Well-1 & -15.3 & -110 \\
\hline HTH-3 & -14.2 & -104 \\
\hline Well 2 & -14.2 & -103 \\
\hline UE-15d & -14.2 & 108 \\
\hline UElc & -13.9 & -104 \\
\hline UE16f & -13.5 & -107 \\
\hline \multicolumn{3}{|l|}{ Volcanic Wells } \\
\hline U20n & -14.9 & -113 \\
\hline UE-18r & -15.0 & -110 \\
\hline UE-18t & -13.8 & -105 \\
\hline PM3 & -14.8 & -115 \\
\hline Water Well 20 & -15.5 & -111 \\
\hline Well 8 & -14.0 & -104 \\
\hline UE-19c & -15.4 & -111 \\
\hline U19ba & -14.0 & -104 \\
\hline UE-4t & -13.8 & -101 \\
\hline \multicolumn{3}{|l|}{ Well ER-12-1 } \\
\hline ER-12-1 & -12.4 & -94 \\
\hline \multicolumn{3}{|l|}{ Alluvial Wells } \\
\hline Test Well B & -14.1 & -105 \\
\hline \multicolumn{3}{|c|}{$\underline{\text { Rainier Mesa Volcanic Seeps }}$} \\
\hline U12n.03 & -13.3 & -97 \\
\hline U12n.03 & -12.4 & -92 \\
\hline U12n.03 & -13.4 & -99 \\
\hline U12n.03 & -13.5 & -95 \\
\hline U12n.03 & -13.4 & -97 \\
\hline U12n.05 & -12.9 & -94 \\
\hline U12n.05 & -13.0 & -93 \\
\hline U12n.05 & -13.0 & -95 \\
\hline U12n.05 & -13.0 & -94 \\
\hline U12n.05 & -12.8 & -93 \\
\hline
\end{tabular}


service tables for stacked logs, lack of calibration tables for the $0.445 \mathrm{~m}$ (17-1/2in) diameter borehole, and lack of written procedures for running these logs in the field.

Hydrologic investigations consisted of water level monitoring, flow logging, aquifer tests, and drill-stem tests. The results indicated that the static composite fluid level in well ER-12-1 is $469 \mathrm{~m}$ ( $1540 \mathrm{ft}$ ) below land surface. Drill-stem tests and flow logs determined that the lower two intervals in the well were under-pressured relative to the upper zones by approximately $396 \mathrm{~m}(1300 \mathrm{ft})$. Aquifer tests, drill-stem tests, and flow logs determined that the transmissivity of the well ranged from $7 \times 10^{-6}$ to $4 \times 10^{-4} \mathrm{~m}^{2} / \mathrm{s}$, with the most transmissive zone being 518 to $554 \mathrm{~m}$ (1700 to 1820 $\mathrm{ft}$ ) below land surface followed by the 914 to $963 \mathrm{~m}$ (3000 to $3160 \mathrm{ft}$ ) interval. The pressure differential between these zones allowed for substantial cross-flow to occur while the well was open.

Two types of geochemical samples were acquired from this well. Water quality samples taken during drilling and testing indicated very few problems associated with the well. Those identified consisted of elevated concentrations of volatile and semivolatile organics and metals associated with the drilling process. Geochemical characterization samples were taken only from the uppermost zone, 518 to $554 \mathrm{~m}$ ( 1700 to $1820 \mathrm{ft}$ ). The results from this sample indicated anomalous chemistry, which is most likely due to residual drilling fluids remaining in the borehole. Trace levels of tritium detected in the upper borehole may be attributed to contamination by drilling fluid, water recharging via the nearby U12e tunnel ponds, or water flowing from the underground nuclear tests conducted in Rainier Mesa.

\section{REFERENCES}

Atlas Wireline Services, 1985, "Log Interpretation Charts," Atlas Wireline Services report.

Atlas Wireline Services, 1988, "Logging Procedures Manual for NTS" (Per Contract SC-TS-84-197) Atlas Wireline Services internal report.

Cole, J.C., A.G. Harris, M.A. Lanphere, C.E. Barker and R.G.Warren, 1993, The case for pre-middle Cretaceous extensional faulting in northern Yucca Flat, southwestern Nevada, Geological Society of America Abstracts with Programs, Vol. 25, no. 5, p. 22.

Cole, J.C., 1991 unpublished, "Pre-drilling Geologic Summary for Characterization Well E-Tunnel Access' (ER-12-1),"U.S. Geological Survey Unpublished Report, 7 p.

Cooper, H.H, J.D. Bredehoeft and I.S. Papadopulos, 1967, Response of a finite-diameter well to an instantaneous charge of water, Water Resources Res., Vol. 3, p 263-269.

Cooper, H.H. Jr. and C.E. Jacob. 1946, A generalized graphical method for evaluating formation constants and summarizing well-field history, American Geophysical Union Transactions, 27 , No. 4, p 526-534.

Desert Research Institute, 1990, Cultural Resources Reconnaissance Short Report SR081090-1, Quaternary Sciences Center, Desert Research Institute, Las Vegas and Reno, NV. 
Dickey, D.D. and F.A. McKeown, 1959, Geology of Dolomite Hill, Nevada Test Site, Nye County, Nevada, U.S. Geological Survey Trace Elements Investigations Report TEI-755, 64 p.

Drellack, S.L., Jr., L.B. Prothro, P.H. Thompson and R.L. McCall, 1991, "Preliminary Geology and Drill Hole Data Report for Groundwater Characterization Well ER-12-1, Nevada Test Site, Nye County, Nevada," Raytheon Services Nevada report, 77 p.

Epstein, A.G., J.B. Epstein and L.D. Harris, 1977, Conodont color alteration - an index to organic metamorphism, U.S. Geological Survey Professional Paper 995, 27 p.

Gibbons, A.B., E.N. Hinrichs, W.R. Hansen and R.W. Lemke, 1963, Geology of the Rainier Mesa Quadrangle, Nye County, Nevada, U.S. Geological Survey Geological Quadrangle Map GQ-215.

Gupta, V.K. and G.R. Walter, (1985) unpublished, Accounting for time variable pumping in analysis of pumping tests, Hydro Geo Chem Inc., 1430 N. 6th Ave, Tucson, AZ.

Harris, A.G., B.R. Wardlaw, C.C. Rust and G.K. Merrill, 1980, Maps for assessing thermal maturity (conodont color alteration index maps) in Ordovician through Triassic rocks in Nevada and Utah and adjacent parts of Idaho and California, U.S. Geological Survey Miscellaneous Investigations Map I-1249, scale 1:2,500,000.

Lai, R.Y. and C. Su, 1974, Non-steady flow to a large well in a leaky aquifer, Journal of Hydrology, Vol. 22, p 333-345.

Lawrence Livermore National Laboratory, 1993, Isotopic Analysis of Groundwater from Monitoring Well Er-12-1. Letter Report to Steve Lawrence DOE/ERP Technical Program Officer of UGTA RIFS, Dec. 15, 1993.

Kruseman, G.P. and N.A. de Ridder, 1992, Analysis and Evaluation of Pumping Test Data, International Institute for Land Reclamation and Improvement Pub. 47, Wageningen, the Netherlands, $365 \mathrm{p}$.

Maldonado, F., S.G. Steele and D.R. Townsend, Supplementary Lithologic Logs of Selected Vertical Drill Holes in Area 12, Nevada Test Site, U.S. Geological Survey.

Miller, D.R., 1970, Lithologic logs and stratigraphic identification for vertical drill holes in Area 12, Nevada Test Site, U.S. Geological Survey Report Area 12-27, 34 p.

Millet, M.R. and D.J. Felske, 1989, LLNL NTOD Geophysics Logging Procedures, GEO-89-01.

Moench, A.F., 1984, Double-porosity models for a fissured groundwater reservoir with fracture skin, Water Resources Research, Vol. 20, p. 831-846.

Naeser, C.W. and F. Maldonado, 1981, Fission-track dating of the Climax and Gold Meadows stocks, Nye County, Nevada, In Short Contributions to Geochronology, U.S. Geological Survey Professional Paper 1199-E, p. 45-47. 
Osmond, J.C., 1954, Dolomites in Silurian and Devonian of east-central Nevada, Bulletin of the American Association of Petroleum Geologists, Vol. 38, No. 8, p. 1911-1956.

Pawloski, G.A. and R.C. Carlson, 1992, Preliminary geophysical logging data report for Ground Water Characterization well ER-12-1, Nye County, Nevada Test Site, Lawrence Livermore National Laboratory Report, February 1992, 69 p.

Papadopulos, S.S., J.D. Bredehoeft and H.H. Cooper, 1973, On the analysis of slug test data: Water Resources Res., Vol. 9, p. 1087-1089.

Russell, C.E., 1991 unpublished, "Hydrogeologic Summary for Groundwater Characterization Well ER-12-1 (U12e Tunnel Access)", Desert Research Institute unpublished report, Reno and Las Vegas, NV, $14 \mathrm{p}$.

Russell, C.E., L. Gillespie, D. Gillespie, 1993, Geochemical and hydrologic characterization of the effluent draining from U12e, U12n, and U12t tunnels, Area 12, Nevada Test Site. Desert Research Institute Publication 45105, Reno and Las Vegas, NV, p 24.

Schlumberger Well Services, 1992, Formation MicroScanner Report for ER-12-1, Shlumberger Well Services, 5000 Gulf Freeway, P.O. Box 2175, Houston, Texas 77001.

Schoff, S.L. and I.J. Winograd, 1961, Hydrologic significance of six core holes in carbonate rocks of the Nevada Test Site, U.S. Geological Survey Trace Elements Investigations Report TEI-787, $97 \mathrm{p}$.

Stehfest, H., 1970, Numerical inversion of Laplace transform, Communications of ACM, Vol. 13, No. $1, \mathrm{p} 47-49$.

Theis, C.V., 1935, The relation between the lowering of the piezometric surface and the rate and duration of discharge of a well using ground-water storage, Trans. Amer. Geophys. Union, Vol. 16, p $519-524$.

Thordarson, W., 1965, Perched groundwater in zeolitized-bedded tuff, Rainier Mesa and vicinity, Nevada Test Site, Nevada, U.S. Geological Survey Trace Elements Investigation Report TEI-862, $89 \mathrm{p}$.

Waddell, R.K., J.H. Robison and R.K. Blankennagel, 1984, Hydrology of Yucca Mountain and vicinity, Nevada-California: Investigative Results through mid-1983, U.S. Geological Survey Water-Resources Investigations Report 84-4267, 40 p.

Winograd, I.J. and W. Thordarson, 1975, Hydrogeologic and Hydrochemical Framework, South-Central Great Basin, Nevada-California. with Special Reference to the Nevada Test Site, U.S. Geological Survey Professional Paper 712-C, 119 p. 
APPENDIX A

GEOLOGIC PREDICTION REPORT 



\title{
DOE ENVIRONMENTAL RESTORATION PROGRAM GROUND WATER CHARACTERIZATION DRILLING PROGRAM
}

\author{
PRE-DRILLING GEOLOGIC SUMMARY \\ FOR CHARACTERIZATION WELL
}

"E-TUNNEL ACCESS" (ER12-1)

\author{
James C. Cole \\ U.S. Geological Survey \\ Denver, Colorado
}

4 May, 1991

\section{Introduction}

The first ground water characterization well is proposed to be drilled on the east slope of Rainier Mesa in south-central Area 12 of the Nevada Test Site. The site is about 400 feet south of the paved access road to the (inactive) Etunnel complex and adjacent to an unimproved jeep trail in the bottom of a small northeast-trending draw.

\begin{tabular}{|ll|}
\hline Hole Name: & E-Tunnel access \\
\hline Hole Designation: & ER12-1 \\
\hline Site Coordinates: & N. 886,650; E. 640,600 \\
\hline Surface Elevation: & 5810 feet \\
\hline Design Depth: & 3500 feet \\
\hline Water Table Depth: & \pm 1600 feet (est.) \\
\hline Surface Geology: & Simonson Dolomite \\
\hline
\end{tabular}

The coordinates listed above are about 500 feet northeast of those $(N$. 886,400 ; E. 640,200 ) listed in the memorandum of 10 January 1991 ("Well Site Review and Geology Meeting") issued prior to the first meeting of the ground water characterization planning group on 16 January 1991 . These coordinates were scaled from the 1:12,000-scale USGS topographic map (Rainier Mesa SW) based on the location identified by flagged lath stakes that were present when the site was visited on 29 March 1991.

This location was selected by DRI and USGS (various correspondence in 1990) to explore the geologic and hydrologic conditions of Paleozoic carbonate rocks in a down-gradient position from expended underground test sites within the Rainier Mesa complex. The collar position was chosen to allow penetration of dolomite through the unsaturated zone and for several hundred feet below the water table, and to reach the footwall of the Tongue Wash fault (most likely Eleana Formation clastic rocks) within the 3500 foot design depth. 


\section{Background}

Systematic geologic mapping of the area around the E-Tunnel complex east of Rainier Mesa was completed in the late 1950's and published at 1:24,000scale for the Rainier Mesa quadrangle (Gibbons and others, 1963). The hillslope immediately west of the ER12-1 site was more closeiy investigated in conjunction with drilling of the "Dolomite Hill" core hole in 1959. This hole penetrated 1200 feet of unsaturated dolomite; core recovery ranged from fair to poor, and approximately 27 boxes of NX core are cataloged at the USCS Coro-Libraxy facility in Mercury (presently stored in Area 25 at the RMAD building). A detalled geologic map of the area and a detailed lithologic log for that hole were reported by Dickey and McKeown (1960). An interpretation of geophysical logs for the Dolomite Hill hole and the results of limited mineralogical and chemical analyses were presented by Roach and others (1960?).

An exploratory core hole designated U12e.M-1 was also drilled in 1959 from an alcove at elevation 6158 within the E-Tunnel complex. Good to excellent core recovery was obtained by the continuous wireline method, but current records do not indicate where these materials presently reside. No geophysical logs were run. A lithologic log for U12e.M-1 is reported by Schoff and Winograd (1961), along with the resuits of water level measurements, bailing and pumping tests, and related hydrologic investigations for this and the Dolomite Hill hole. Thordarson (1965) summarized the hydrologic observations for these holes that penetrated Paleozoic rocks as part of a comprehensive report on perched ground water in the Rainier Mesa environs.

Drill holes U12e.06 A and U12e.06 B entered Paleozoic dolomite but did not penetrate more than 100 feet. Hole U12e.06-1 R/C, however, penetrated 755 feet of dolomite (McArthur and Skrove, 1962; Miller, 1970); the existence of geophysical logs and core samples is unknown.

\section{Stratigraphy}

Stratigraphic relationships and nomenclature for the Paleozoic rocks exposed along the western margin of the Yucca Flat basin have been the subject of renewed investigation in recent years (Peter Guth, written communication, 1988; Alan Titus, written communication, 1989; Caskey and Schweickert, 1989; Cole and others, 1990 and unpublished mapping). For the Devonian and Mississippian units of interest surrounding the ER12-1 site, the following table summarizes current usage for named stratigraphic units: 


\begin{tabular}{|c|c|}
\hline MDe Eleana Fm (units A-J) & MDe Eleana Fm (units A-J) \\
\hline \multicolumn{2}{|c|}{ [not in stratigraphic contact in this area] } \\
\hline Dg Guilmette Fm & $\begin{array}{l}\text { Did or Dd Devils Gate } \\
\text { Limestone }\end{array}$ \\
\hline Ds Simonson Dolomite & Dnu or Dn Nevada Fm (upper) \\
\hline Dsca "cherty-argll. unit" & Dnl (part) Nevada Fm (lower) \\
\hline DSs Sevy Dolomite & $\begin{array}{l}\text { DSOI Dolomite and limestone: } \\
\text { also. Spotted Ronge dolomite } \\
\text { units D-F }\end{array}$ \\
\hline SI Laketown Dolomite & SI Laketown Dolomite \\
\hline
\end{tabular}

The dolomitic rocks mapped at the surface on the east side of Rainier Mesa by Dickey and McKeown (1960) and by Gibbons and others (1963) correspond to the Simonson Dolomite, the "cherty-argillaceous unit", and (possibly) the Sevy Dolomite. The three subunits of the Nevada Fm shown on the Rainier Mesa quadrangie map (Dda, Ddb, and Ddc) correspond most closely with the middle and upper parts of the regionally defined Simonson Dolomite (Osmond, 1954). Osmond's lowermost unit of "tan, coarse dolomite" and the "brown, cliffforming biostromal dolomite" do not appear to be mappable entities in the NTS area.

Devonian rocks crop out at several widely separated localities at NTS and physical correlation between sections is not possible. No systematic study has been conducted in these rocks to determine internal stratigraphic relations, fossil contents, or depositional environments, and thus the structural and(or) hydrologic connections between the individual outcrop blocks are not determined. In general, however, there is overall similarity in terms of internal lithology, thickness, and fossil content between the exposed sections: 1) North of Oak Canyon; 2) East of Rainier Mesa; 3) Mine Mountain; 4) Shoshone Mountain; and 5) at Mercury Ridge. (NOTE: As pointed out by Rogers and Noble, 1969, two dissimilar Devonian sequences in the Oak Spring Butte quadrangle suggest that a major pre-Tertiary structure is present between the limestone-rich section along Carbonate Wash beneath the basal Eleana Formation and the more typical dolomite-rich section north of Oak Canyon). In summary, the typical Devonian units are:

Guilmette Limestone

about 1000 feet thick; thick, alternating beds of medium-gray limestone and sandy limestone, with local thin beds of brownish quartz sand and limy quartzite; dolomite beds interfinger near base and also dominate in a 200-foot thick zone below the uppermost quartzite beds at Shoshone Mountain; corals, crinoids, and some brachiopods and algal fossils are typical but not common

Simonson Dolomite

thickness appears to range between about 935 feet (Mercury Ridge) and more than 2000 feet (east of Rainier Mesa); chiefly consists of well bedded dolomite of various shades of gray; limestone is untypical and sandy beds are generally sparse except near the base and in 
the middle; dolomitized fossil brachiopods, stromatoperoid algal masses, crinoids, and tubular forms (áescribed as "spaghetti coral") are common and form substantial concentrations in biostromal beds, particularly in the middle and upper parts of the formation; the distinctive and time-restricted Stringocephalus brachiopod is exceptionally abundant in thin beds near the top of the formation at Mercury Ridge, Shoshone Mountain, and in Carbonate Wash. A general three-fold subdivision seems to be recognizable:

- Upper variegated dolomite ( 400 to 600 feet thick, but as thick as 1500 feet at Dolomite Hill); well-bedded, generaily dark-gray in aspect, fossil-rich

- Middle tan-gray dolomite (250 to 300 feet thick); thick-bedded with platy internal laminations, more uniform in overall color than units above and below; somewhat sandy or silty, especially near base; fossils less common

- Lower variegated dolomite (180 to about 600 feet thick); well bedded; light to medium gray with discrete dark-gray dolomite beds; sandy and brownish in lower part

"Cherty-argillaceous unit" about 40 to 120 feet thick; brownish sandy, silty, and chert-bearing thinbedded dolomite; chert nodules are red, brown, or black and irregularly shaped; silty dolomite is olive-drab on fresh surfaces Sevy Dolomite but weathers yellow-gray

about 900 feet thick; typically poorly bedded and homogeneous, light mouse-gray, coarse-grained; commonly brecciated; fossils extremely rare and generally consist of dolomitized ghosts of corals(?) and brachiopods; the middle third of the Sevy tends to be a bit darker and indistinctly banded, but this feature is most apparent from a distance

Presence of the Early Devonian Sevy Dolomite in the E-Tunnel area is uncertain. Previous investigators (Dickey and McKeown, 1960; Gibbons and others, 1963) have tentatively identified the light-gray, indistinctly bedded dolomites in the lower hanging wall of the Tongue Wash fault with similarlooking dolomites around NTS that are approximately Sevy-age or older ("DSOI"). However, examination of these rocks on 29 March 1991 shows that some of the indistinct bedding is the result of fault-induced brecciation, and that dark-gray dolomites and sparse fossil crinoid fragments are present (both are atypical of the Sevy). The brown-weathering silty and cherty beds at the bottom of the exposed section next to the Tongue Wash fault are most like the "cherty-argillaceous unit" Dsca that marks the faint regional unconformity between the Sevy and Simonson dolomites (Osmond, 1954), and so it is likely that the hillslope above is lower Simonson rather than Sevy. This interpretation is also more consistent with structural relations described in the next section.

The Mississippian Eleana Formation exposed in the Eleana Range east of Rainier Mesa is expected to be encountered at depth in ER12-1 in the footwall of the Tongue Wash fault. Because of structural complications described below, it is difficult to predict which subunits of the Eleana will be penetrated with great 
certainty. However, the gross anticlinal structure of the Eleana Range indicates that the middle and lower parts of the formation are most likely (units D and lower), and so laminated fine-grained quartzite, siltstone, and thin chert-pebble conglomerate beds are expected (Poole and others, 1961). In this regard, 1 differ with the interpretation of Gibbons and others (1963) that the west-dipping beds of the Eleana east of Tongue Wash are overturned; examination of conglomerate beds in this area are inconclusive about the sense of stratigraphic facing, but several instances of graded bedding suggest that the section is right-side-up.

\section{Structure}

Most of the structural geology on the east side of Rainier Mesa that is relevant to expected conditions beneath the ER12-1 site pertains to the configuration of the Paleozoic rocks. These are exposed along the Tongue Wash valley, in the Eleana Range, and west of the drill site up to Dolomite Hill, but these outcrops lack lateral continuity to the southwest and northeast. Faulting, bedding, and related features must be inferred at depth because they cannot be confidently projected downward from the surface. Uncertainty about some of the Devonian rocks, and lack of reliable stratigraphic indicators in the Eleana Formation on the west flank of the Eleana Range aiso add to the uncertainty about structural configurations.

The Tertiary volcanic rocks are largely draped over the pre-existing topographic surface formed by the Paleozoic rocks. This area of the Nevada Test Site has been tectonically stable, for the most part, since the onset of major volcanic activity about 15 million years ago (Sawyer and others, 1990). Stability is indicated by the gentle dips of voicanic layering, by the neariy uninterrupted deposition of volcanic ash in the Rainier Mesa area in the period $15 \mathrm{Ma}$ to 11 $\mathrm{Ma}$, and by paleomagnetic investigations by Hudson (1990). Structural warps and high-angle faults do affect the Tertiary section in this area, but the magnitude of deformation is small in comparison to that recorded in the Paleozoic rocks (Minor, 1990).

The west-dipping fault that is exposed on the west side of Tongue Wash and juxtaposes footwall Mississippian Eleana Formation with hangingwall Devonian carbonate rocks has long been interpreted as a thrust (for example, Gibbons and others, 1963) and inferred to have formed during the Mesozoic Sevier orogeny. This "CP thrust" of Carr (1974) and others does not appear to be supported by outcrop evidence. Examination of the fault zone shows numerous grooved slip surfaces in both wallrocks that dip westward between $42^{\circ}$ and $48^{\circ}$. Slickenlines consistently plunge toward the northeast at shallow inclinations (four measured values between $14^{\circ}$ and $20^{\circ}$; fig. 1), and subtle asymmetries on these surfaces suggest the sense of movement was sinistralreverse (M. Hudson, oral commun., 1989). At the outcrops north of the $E$ Tunnel access road just-west of the Stockade Wash Road (fig. 1), the silicified fault zone is notably sinuous as it is exposed to the north in a manner that suggests large-scale, northeast-plunging grooves and swales.

The Eleana quartz sandstones, siltstones, and chert-pebble conglomerates adjacent to the fault are relatively unsheared, but the dolomites of the hangingwall tend to be shattered, veined with silica and calcite, and locally stained with iron oxides. These types of features are inconsistent with observations along well-known thrust faults of the Sevier system (Cole and others, 1990). 
Additional surface reconnaissance east of the ER12-1 site indicates that the northeast-trending fault shown on the Rainier Mesa quadrangle map (Gibbons and others, 1963) is not a large-displacement high-angle structure. As shown on Fig. 1, and in section on Fig. 2, this fault dips moderately east and is marked by a distinctive, 3-foot-thick breccia zone of gray dolomite fragments and yellow-weathering silica veins; talus blocks of this material litter the east-facing slope of the small ridge, which must approximately coincide with the original fault surface. In addition, the argument given above for identification of the hangingwall rocks in this block as lower Simonson Dolomite (rather than Sevy: equivalent; Gibbons and others, 1963) implies that the amount of offset is small (less than about 200 feet, fig. 2). As shown on fig. 1, slip on this fault appears to diminish to the southwest and die out. Similar relations in minor faults within the dolomites were mapped in detail by Dickey and McKeown (1959).

In summary, the Tongue Wash fault is interpreted as a pre-volcanic structure whose last major movement was dominantly strike-slip, although the orientation of the fault surface at the time of slip is not known. The asymmetric anticiinal form of the Eleana in the footwall may have formed simultaneously or later and may preserve a footwall fold related to mid-Tertiary extension (as perhaps with the Eleana anticline in the Mine Mountains; Cole and others, 1990; see also Guth, 1990). Pre-volcanic Tertiary extension is cleariy indicated in this area on the basis of the low-angie fault structures defined at Mine Mountain by Cole and others (1990), by the subsurface detection of stacked structural plates of shattered Devonian(?) dolomite and Eleana siltstone around the Baneberry site (Area 8; U.S. Geological Survey, 1974), and by the presence of out-of-structure fault blocks of Ordovician limestone south of the Area 12 Camp site (Gibbons and others, 1963).

I infer that the Devonian dolomites west of the Tongue Wash fault are dismembered at depth and disconnected from any regional carbonate stratum. Gravity data (Healey and others, 1987) indicate that high-density "basement" rocks are continuous westward beneath Rainier Mesa for about 4 miles where they are probably truncated by the structural wail of the Timber Mountain caldera. These inferred carbonate rocks are probably also truncated toward the south by similar structures at the southwest end of the Eleana Range.

\section{Expected Conditions}

Prior experience with drilling, coring, and hydrologic testing in carbonate rocks at the Nevada Test Site has been mixed. In the particular cases of the Dolomite Hill hole, U12e.06-1 R/C, and U12e.M-1 (all of which were continuously sampled by the wireline method; NX core), recovery was moderate to excellent except where brecciation was widespread. This condition was particularly troublesome at Dolomite Hill, which is substantially closer to the Tongue Wash fault than the E-Tunnel holes, where recovery was less than $30 \%$ for almost $60 \%$ of the hole (Dickey and McKeown, 1959; Schoff and Winograd, 1961). Widespread rock fracturing in U12e.M-1 and Dolomite Hill also led to loss of circulation in several long intervals; large quantities of bentonite was added to the drilling fluid to maintain circulation, and hydrologic test results were compromised (Schoff and Winograd, 1961).

Many breccia zones described from the core had open, vuggy channels and permeable fractures, but other breccias were sealed with calcite cement. In Dolomite Hill, core from the upper and lower thirds of the hole seemed to have 
open fractures and greater inferred permeability than the middle third where calcite fillings were more pervasive, but no interpretation was given. Nonetheless, the various hydrologic tests that were made in Dolomite Hill and U12e.M-1 clearly indicate that rock permeability is controlled by fractures and not by interstitial flow. Even with the uncertain effect of residual bentonite in fractures, fluid levels declined in these holes at rates that imply total formation permeabilities an order of magnitude greater than lab-measured interstitial permeability (Schoff and Winograd, 1961). In order to establish the effect of fracture conditions on ground water behavior, cultings alone are inadequate to establish what kinds of fractures and fracture fillings are present. Adequate samples from continuous core must be obtained.

Drilling in the Eleana Formation should not produce unexpected difficulties, even though it is thin-bedded, closely fractured at the surface, and has exhibited some squeezing behavior. More than $96 \%$ core recovery (NX size) was obtained in UE17e in a 3000 foot hole (Hodson and Hoover, 1979) and UE25a-3 showed similar recovery over 2500 feet at Calico Hills (Maldonado and others, 1979). UE16d and UE16f both experienced problems in some intervals with caving and hole erosion during rotary drilling (3000 feet and 1500 feet, respectively), but hydraulic tests were successfully completed in both holes (Dinwiddie and Weir, 1979).

\section{Expected Geology}

On the basis of surface geology and geometric projections to depth, the following rock units and structures are anticipated to be penetrated during drilling of ER12-1:

\begin{tabular}{|c|c|}
\hline DEPTH, in feet & GEOLOGIC FEATURE \\
\hline $320 \pm 20$ & Base of middle Simonson untt \\
\hline $740 \pm 40$ & Base of Simonson Dolomite \\
\hline $790 \pm 40$ & Bcse of "cherty-argill" unit \\
\hline $1000 \pm 100$ & $\begin{array}{l}\text { Intersection with minor east- } \\
\text { dipping foult }\end{array}$ \\
\hline $1650 \pm 200$ & Potentiometric surface \\
\hline $2070 \pm 180$ & $\begin{array}{l}\text { Foulted base of Sew Dolomite } \\
\text { top of Eleana Formation } \\
\text { Plercement of Tongue Wash } \\
\text { fault }\end{array}$ \\
\hline 3500 & $\begin{array}{l}\text { Bottom of hole: lower Eleano } \\
\mathrm{Fm} \text {. }\end{array}$ \\
\hline
\end{tabular}




\section{References Cited}

Carr, W.J., 1974, Summary of tectonic and structural evidence for stress orientation at the Nevada Test Site: U.S. Geological Survey Open-File Report 74-176, 48 p., map at 1:48,000 scale.

Caskey, S.J., and Schweickert, R.A., Mesozoic west-vergent thrust in the CP Hills, Nevada Test Site, Nye County, Nevada: Geological Society of America Abstracts with Programs, vol. 21, no. 5, p. 64.

Cole, J.C., Wahl, R.R, and Hudson, M.R., 1990, Structural relations within the Paleozoic basement of the Mine Mountain block; Implications for interpretation of gravity data in Yucca Flat, Nevada Test Site: Proceedings of the Fifth Symposium on the Containment of Underground Nuciear Explosions, Santa Barbara; vol. 2, p. $431-456$

Dickey, D.D., and McKeown, F.A., 1959, Geology of Dolomite Hill, Nevada Test Site, Nye County, Nevada: U.S. Geological Survey Trace Elements Investigations Report TEI-755, 64 p.

Dinwiddie, G.A., and Weir, J.E., Jr., 1979, Summary of hydraulic tests and hydrologic data for holes UE16d and UE16f, Syncline Ridge area, Nevada Test Site: U.S. Geological Survey Report USGS-1543-3, 21 p.

Gibbons, A.B., Hinrichs, E.N., Hansen, W.R., and Lemke, R.W., 1963, Geology of the Rainier Mesa Quadrangle, Nye County, Nevada: U.S. Geological Survey Geologic Quadrangle Map GQ-215, scale 1:24,000

Guth, P.L., 1990, Bedrock geologic map of the Indian Springs 1:100,000-scale quadrangle, Nevada: U.S. Geological Survey Open-Ffle Report 90-???, 32 p., scale 1:100,000.

Hodson, J.N., and Hoover, D.L., 1979, Geology of the UE17e drill hole, Area 17. Nevada Test Site: U.S. Geological Survey Report USGS-1543-2, 36 p.

Hudson. M.R, 1990, Paleomagnetic evaluation of the Yucca-Frenchman flexure, southern Nevada: Proceedings of the Fifth Symposium on the Containment of Underground Nuclear Explosions, Santa Barbara; vol. 2, p. $417-430$.

Maldonado, F., Muller, D.C., and Morrison, J.N., 1979, Preliminary geologic data of the UE25a-3 exploratory drill hole, Nevada Test Site, Nevada: U.S. Geological Survey Report USCS-1543-6, 43 p.

McArthur, R.D., and Skrove, J.W., 1962, ...: Lawrence Radiation Laboratory report CN-1-62.

Miller, D.R., 1970, Lithologic logs and stratigraphic identification for vertical drill holes in Area 12, Nevada Test Site: U.S. Geological Survey Report Area $12-27,34 \mathrm{p}$.

Minor, S.A., 1990, Paleostress investigation near Rainier Mesa, Nevada Test Site: Proceedings of the Fifth Symposium on the Containment of Underground Nuclear Explosions, Santa Barbara; vol. 2, p. 457-482. 
Osmond, J.C., 1954, Dolomites in Silurian and Devonian of east-central Nevada: Bulletin of the American Association of Petroleum Geologists, vol. 38, p. 1911-1956.

Poole, F.G., Houser, F.N., and Orkild, P.P., 1961, Eleana Formation of Nevada Test Site and vicinity, Nye County, Nevada: U.S. Geological Survey Professional Paper 424-D, p. D104-D111.

Roach, C.H., Izett, G.A., and Roller, J.C., 1959, Interpretation of geophysical logs of the Granite U-15A, Dolomite Hill No. 1, and Marble No. ME-2 drill holes, Nevada Test Site, Nye County, Nevada: U.S. Geological Survey Trace Elements Investigations Report TEI-???, ? p.

Rogers, C.L., and Noble, D.C., 1969, Geology of the Oak Spring Butte guadrangle, Nye County, Nevada: U.S. Geological Survey Geologic Quadrangle Map GQ-822, scale 1:24,000.

Sawyer, D.A., Fleck, R.J., Lanphere, M.A., Warren, RG., and Broxton, D.E., 1990, Episodic volcanism in the southwest Nevada volcanic field: new ${ }^{40} \mathrm{Ar} /{ }^{39} \mathrm{Ar}$ geochronologic results: Transactions of the American Geophysical Union (EOS), vol. 71, p. 1296.

Schoff, S.L, and Winograd, I.J., 1961, Hydrologic significance of six core holes in carbonate rocks of the Nevada Test Site: U.S. Geological Survey Trace Elements Investigations Report TEI-787, $97 \mathrm{p}$.

Thordarson, W., 1965, Perched ground water in zeolitized-bedded tuff, Rainier Mesa and vicinity, Nevada Test Site, Nevada: U.S. Geological Survey Trace Elements Investigations Report TEI-862, 90 p.

U.S. Geological Survey, 1974, Results of exploration of Baneberry site, early 1971: U.S. Geological Survey Report 474-145, 94 p.

Weir, J.E., Jr., and Hodson, I.N., 1979, Geohydrology of hole UE17a, Syncine Ridge area, Nevada Test Site: U.S. Geological Survey Report USCS-1543-4, $21 \mathrm{p}$. 


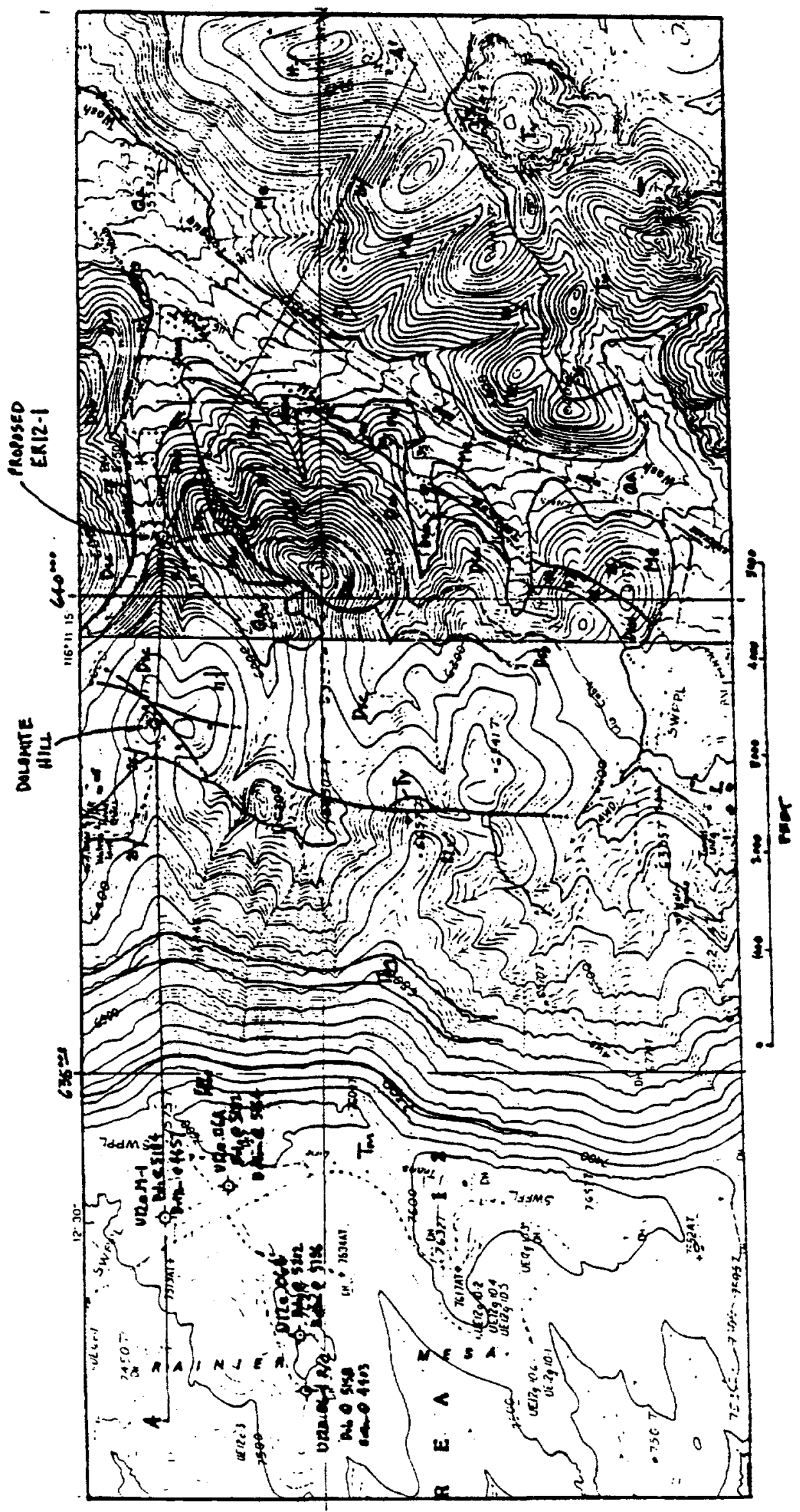




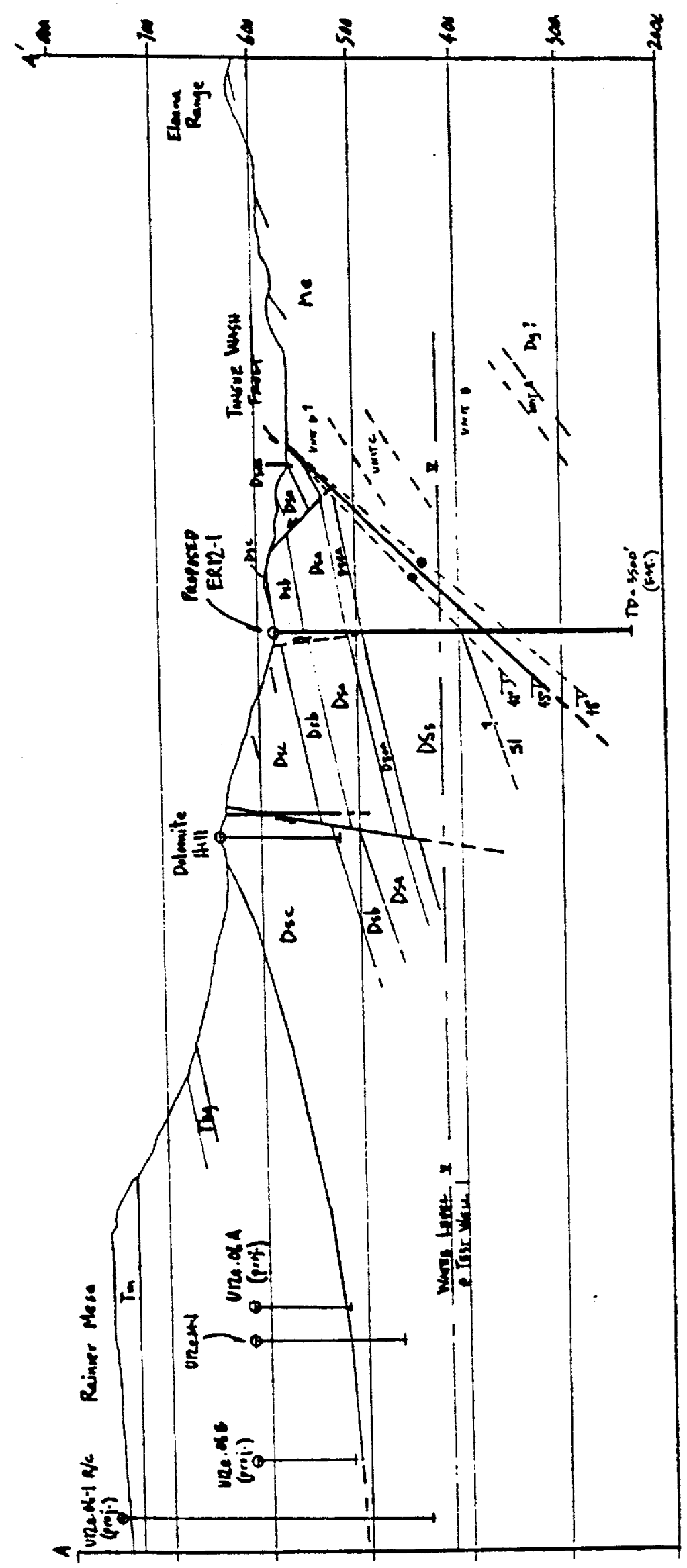



APPENDIX B

HYDROLOGIC PREDICTION REPORT 


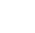




\title{
HYDROGEOLOGIC SUMMARY FOR GROUNDWATER CHARACTERIZATION WELL ER-12-1 (U12E TUNNEL ACCESS)
}

\author{
by \\ Desert Research Institute \\ University of Nevada System
}

prepared for

Environmental Restoration and Waste Management Division U.S. Department of Energy

Field Office, Nevada

Las Vegas, Nevada

August 1991 


\section{CONTENTS}

FIGURES

TABLES

PURPOSE AND SCOPE

OBJECTIVE AND RATIONALE FOR THE ER-12-1 DRILLHOLE

LOCATION AND SETTING

HYDROGEOLOGY

Geology

Hydrology

Fluid Levels

Transmissivities

Vertical Gradients

Geochemistry

SUMMARY

REFERENCES

\section{FIGURES}

1. Location of ER-12-1 drill site.

2. Composite potentiometric surface for the Nevada Test Site and subbasin boundaries.

3. Drill site surface structure and geology.

4. Drillhole locations and water-level elevations.

5. Aquifer test in dolomite in TW-1.

6. Recovery of water level after slug removal in UE-16d, Eleana Formation ( $\mathrm{T}=\mathrm{gpd} / \mathrm{ft}$ ).

7. Recovery of water level after slug injection, Eleana Formation at $\mathrm{UE}-16 \mathrm{f}(\mathrm{T}=0.1 \mathrm{gpd} / \mathrm{ft})$.

\section{TABLES}

1. Anticipated geology.

2. Well construction and static water-level data.

3. Aqueous geochemistry for Test Well 1. 


\section{HYDROGEOLOGIC SUMMARY FOR \\ GROUNDWATER CHARACTERIZATION WELL ER-12-1 \\ (U12E TUNNEL ACCESS)}

\section{PURPOSE AND SCOPE}

This document summarizes pertinent hydrogeologic, hydraulic and geochemical data from wells relatively close and hydrogeologically similar to the ER-12-1 drill site. It also serves as a predictive report of the most probable hydrogeologic conditions that may be encountered, and is intended to be used as a guide for weil design and field sample planning. In addition, this report will act as a baseline for updating the conceptual hydrogeologic model of Rainier Mesa, utilizing data gathered from the ER-12-1 borehole.

\section{OBJECTIVE AND RATIONALE FOR THE ER-12-1 DRILLHOLE}

The location of ER-12-1 was chosen to explore the hydrogeology of Paleozoic carbonates potentially downgradient from underground nuclear tests conducted within Rainier Mesa. This location is also ideal to evaluate the effect of infiltration/evaporation ponds on the regional groundwater flow system.

The drillhole was located and designed to intersect approximately $2100 \mathrm{ft}$ of unsaturated and saturated Devonian dolomites, the Tongue Wash Fault, at a depth of approximately 2100 $\mathrm{ft}$, and $1400 \mathrm{ft}$ of underlying Eleana Formation. Hydrogeologic data from the Eleana Formation will help to evaluate the effectiveness of this unit as a groundwater flow boundary.

\section{LOCATION AND SETTING}

The ER-12-1 drill site is located at Nevada Central Coordinates N886,666 E640,512 (as built) $\pm 100 \mathrm{ft}$ in Area 12 (Figure 1) near the base of the eastern siope of Rainier Mesa along the U12e tunnel access road where it passes close to the base of Dolomite Hill. The drill site is at an approximate elevation of $5810 \mathrm{ft}$ and is collared in a very thin veneer of alluvium that overlies the Devonian Simonson Dolomite. It is approximately $2000 \mathrm{ft}$ northwest of the surficial expression of the Tongue Wash Fault. a northeast-trending sinistral-reverse fault that dips approximately 45 degrees to the west. The fault brings into conjunction Devonian dolomites in the hanging wall with the Mississippian Eleana Formation in the footwall.

Surface water in the ER-12-1 site locale drains into Tongue Wash, which eventually flows into other ephemeral channels draining east into Yucca Flat, a closed hydrographic basin.

The drill site of ER-12-1 is within the Ash Meadows groundwater subbasin defined by Winograd and Thordarson (1975) and Waddell et al. (1984). Near Rainier Mesa, the boundary between the Ash Meadows and Alkali Flat/Furnace Creek subbasins (Figure 2) has been 


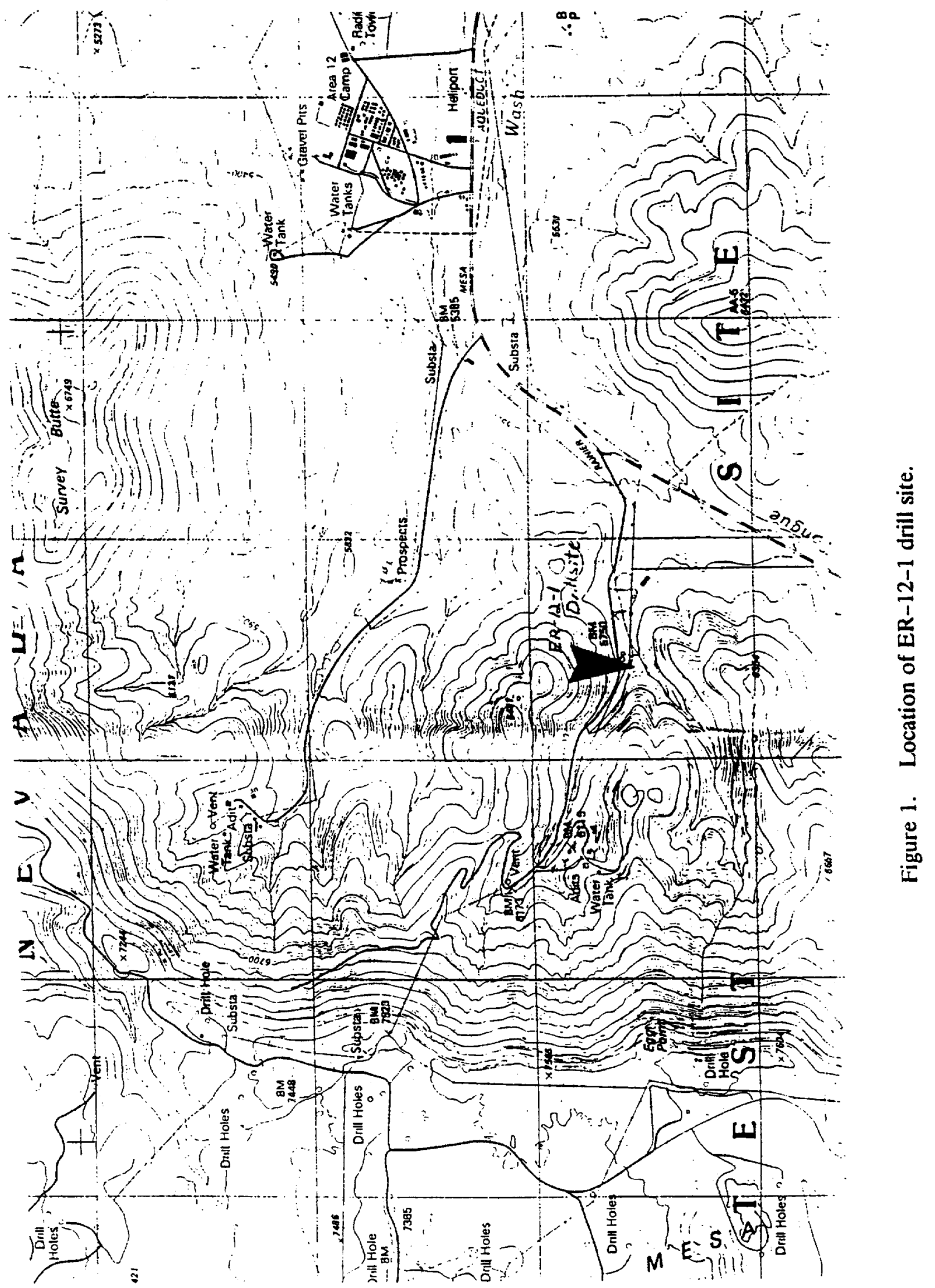


located on the basis of hydrography. It is unlikely that this groundwater subbasin boundary coincides with the hydrographic divide. A more realistic scenario is the groundwater subbasin boundary is defined by the relatively impermeable Eleana Formation (Winograd and Thordarson, 1975). If true, groundwater beneath the ER-12-1 drill site may be draining into the Alkali Flat/Furnace Creek subbasin (via Timber Mountain) with flow ultimately discharging in Alkali Flat and Furnace Creek in Death Valley. If the current boundary, as defined by Winograd and Thordarson (1975) and Waddell et al. (1984), is correct, then the ultimate discharge area for groundwater flow originating near ER-12-1 would be the springs at Ash Meadows and perhaps Death Valley (via Yucca and Frenchman Flats).

The drill site for ER-12-1 is located approximately 1.3 miles southeast and east of underground nuclear tests conducted beneath the caprock of Rainier Mesa. Most of these tests are situated in a saturated groundwater lens whose top is perched approximately 2000 $\mathrm{ft}$ above the estimated regional groundwater table (Thordarson, 1965). In addition, the drill site is located $1500 \mathrm{ft}$ east of the inactive U12e tunnel effluent ponds. These ponds contained

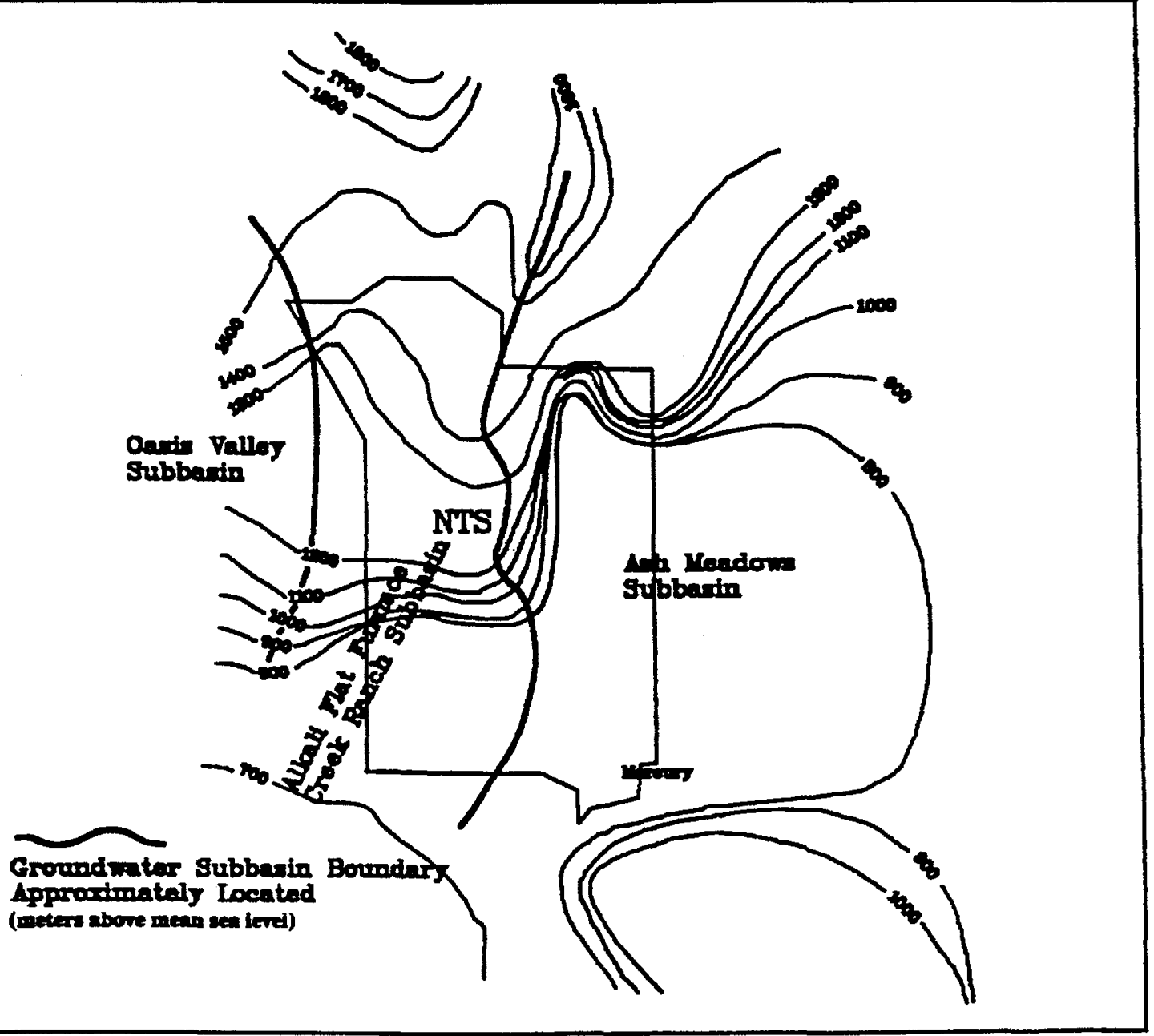

Figure 2. Composite potentiometric surface for the Nevada Test Site and subbasin boundaries (Waddell, Robison, and Blankennagel, 1984). 
tritiated effluent while the tunnel was active and may have introduced contamination into the Paleozoic carbonate rocks locally.

\section{HYDROGEOLOGY}

Pre-existing hydrogeologic data in the vicinity of ER-12-1 are extremely sparse. Hydrogeologic interpolations are dependent upon incomplete data gathered from wells almost three miles distant. Interpolations of hydrogeologic information from these wells are tentative and will probably be revised as drilling and testing at ER-12-1 proceed.

Geology

A generalized interpretation of the geologic units expected to be encountered in ER-12-1 drillhole is presented in Table 1, based upon written communication (Jim Cole, USGS, Denver, Colorado, 5/4/91). ER-12-1 is collared in a thin veneer of alluvium overlying the Simonson Dolomite (Figure 3). The Simonson Dolomite is an approximately $2000 \mathrm{ft}$ thick, well-bedded, fossiliferous dolomite that has been divided into upper, middle and lower subdivisions. The drillhole is anticipated to encounter approximately $740 \mathrm{ft}$ of the Simonson Dolomite.

\begin{tabular}{|c|c|}
\hline \multicolumn{2}{|c|}{ TABLE 1. ANTICIPATED GEOLOGY } \\
\hline $\begin{array}{c}\text { Depth Interval } \\
\text { (feet) }\end{array}$ & Geologic Unit \\
\hline $0-740$ & Simonson Dolomite \\
\hline $740-790$ & Cherty Argillaceous \\
\hline $790-2070$ & Sevy Dolomite \\
\hline $2070-3500$ & Lower Eleana Formation \\
\hline (after Jim Cole, USGS, written communication, 5/4/91) \\
\hline
\end{tabular}

Underlying the Simonson Dolomite is a thin (40 to $120 \mathrm{ft}$ thick) "cherty-argillaceous unit," consisting of a brownish sandy, silty, chert-bearing, thin-bedded dolomite. Approximately $50 \mathrm{ft}$ of this unit is expected to be encountered within the ER-12-1 drillhole.

Underlying the "cherty-argillaceous unit" is the Sevy Dolomite. This dolomite is approximately $900 \mathrm{ft}$ thick and is coarse grained, poorly bedded and homogeneous. The entire section of the Sevy Dolomite is expected to be encountered within the drillhole. A thin section of the Laketown Dolomite may be encountered immediately below the Sevy Dolomite. It is expected that the Tongue Wash Fault will be encountered at the base of the Sevy Dolomite. If true, the lower portion of the Sevy Dolomite and/or the Laketown Dolomite is expected to compose the hanging wall of the Tongue Wash Fault.

The footwall of the Tongue Wash Fault probably consists of the lower members (units D, C, and potentially B) of the Eleana Formation. These lower units consist of laminated, fine-grained quartzite and siltstone with thin chert-pebble conglomerate beds. 


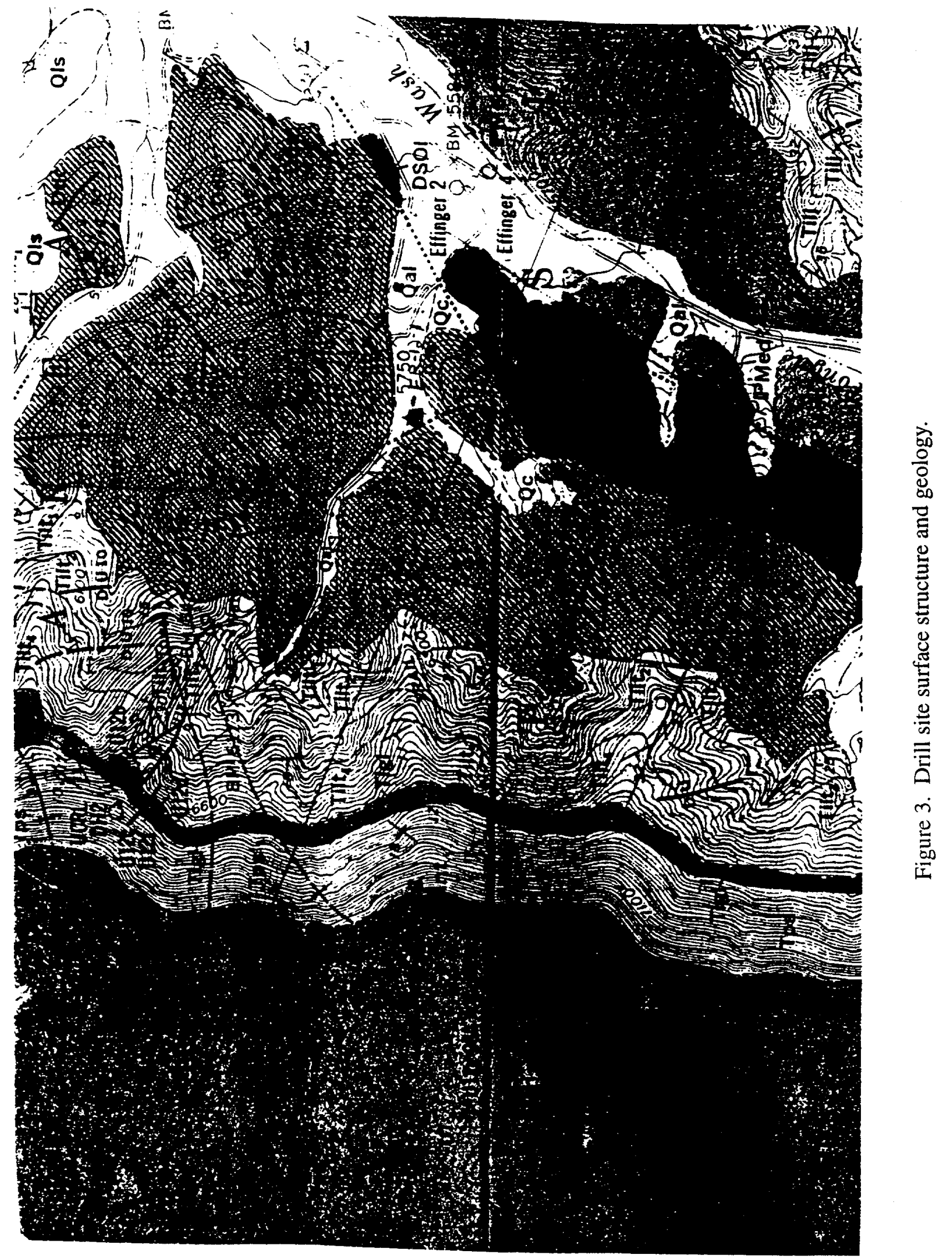




\section{Hydrology}

Fluid Levels

Fluid levels have been measured in several holes in the vicinity of the ER-12-1 drill site: Test Well 1. Hagestad-1, U12e.06-1, U12e.03-1, Dolomite Hill Hole, and U12e.M1 (Figure 4). All of these boreholes were drilled through the Tertiary volcanics into the underlying Devonian dolomites, with the exception of Hagestad-1, which was drilled entirely within Tertiary volcanics. Construction, generalized stratigraphy data, and fluid levels for each hole are presented in Table 2. Fluid levels were declining during fluid--level measurements for all wells except Test Well 1. Fluid leveis within Hagestad-1 were representative of perched water lenses found within confining layers created by Tertiary volcanics. Declining fluid levels within Hagestad-1 were attributed to perched groundwater and residual drilling fluids draining from the formations into a mined cavity beneath the borehole (Thordarson, 1965). Declining fluid levels within Dolomite Hill Hole, U12e.06-1, U12e.M1 and U12e.03-1 were attributed to perched groundwater and/or drilling fluids slowly draining into the underlying unsaturated dolomites. Fluid levels in the boreholes were not representative of static water levels within the Tertiary volcanics nor the Devonian dolomites.

Composite water levels have been measured over time in Test Well 1. Composite water levels are a vertically integrated average of potentiometric heads within both the Devonian dolomites and the Tertiary volcanics. These composite water levels have declined 24 feet over 28 years and have remained relatively stable for at least four years (Table 2). The potentiometric surface for dolomites within Test Well 1 was measured at 4,189 ft. This potentiometric surface is probably representative of carbonate rocks underiying Rainier Mesa and outcrops exposed to the east (Thordarson, 1965). Based upon the generalized geology of the site and fluid levels measured within Test Well 1, it is anticipated that the water table will be encountered at a depth of approximately $1600 \pm 100 \mathrm{ft}$.

Transmissivities

Very few aquifer tests have been conducted within units in the vicinity of ER-12-1. The limited test data are presented along with qualitative observations to generate a reasonable estimation of conditions that may be encountered within the ER-12-1 drillhole. Aquifer tests conducted on $498 \mathrm{ft}$ of dolomites found within Test Well 1 indicate a transmissivity of 3500 $\mathrm{gal} / \mathrm{day} / \mathrm{ft}$ (Figure 5). The units tested were either the Simonson Dolomite or the overlying Guilmette Formation (missing in the geologic section at the ER-12-1 drill site). In addition, the retention of $84 \mathrm{ft}$ of elevated head (drilling fluid) for over seven months within the Dolomite Hill Hole is a qualitative indication of a relatively low transmissivity for the Simonson Dolomite (Schoff and Winograd. 1961). However, in the same drillhole, circulation was lost six times. The most serious loss of circulation occurred in a $15-\mathrm{ft}$-thick section of the Simonson Dolomite, where a maximum of 5,000 gallons of drilling fluid were lost. The loss of drilling fluid is an indication of the presence of relatively permeable sections. Several 


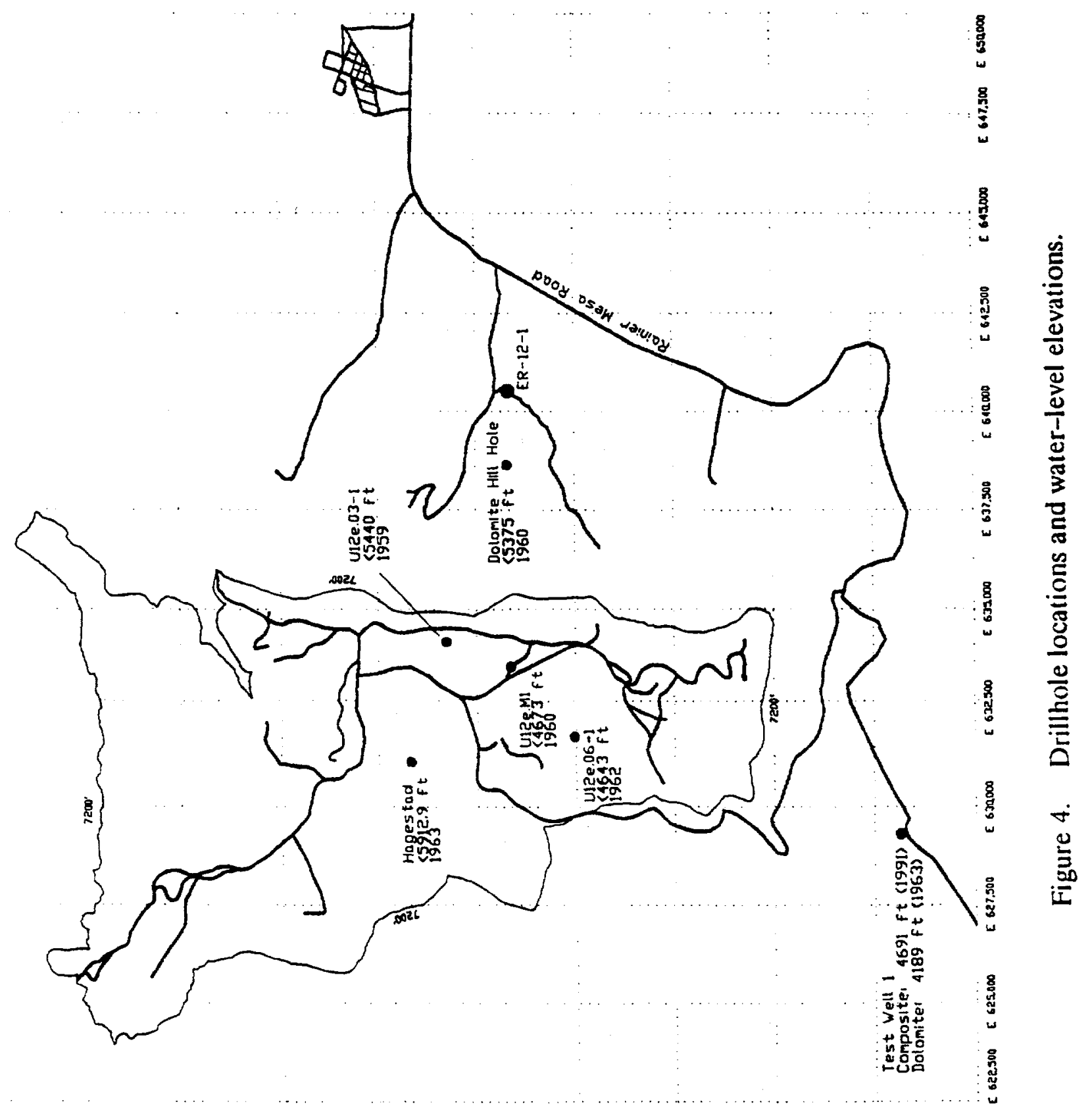

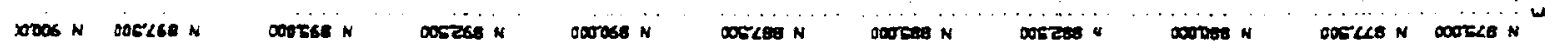




\begin{tabular}{|c|c|c|c|c|c|c|c|c|c|c|}
\hline \multirow[b]{3}{*}{ Well } & & TABLE 2. & WELL & ONSTRI & UCTION AI & VD STATIC II & ATER-LEVEL & DATA & & \\
\hline & \multirow[b]{2}{*}{ Coordinates } & \multirow{2}{*}{$\begin{array}{c}\text { Year } \\
\text { Completed }\end{array}$} & \multirow{2}{*}{$\begin{array}{c}\text { Depth of } \\
\text { Well } \\
\end{array}$} & \multicolumn{2}{|c|}{ Casing } & \multirow{2}{*}{$\begin{array}{c}\text { Perforaled } \\
\text { Zones } \\
\end{array}$} & \multirow{2}{*}{$\begin{array}{c}\text { Formalions } \\
\text { Present }\end{array}$} & \multirow{2}{*}{$\begin{array}{l}\text { Altitude } \\
\text { of Land } \\
\text { Surface }\end{array}$} & \multirow{2}{*}{$\begin{array}{c}\text { Static } \\
\text { Watter } \\
\text { Elevation }\end{array}$} & \multirow{2}{*}{$\begin{array}{c}\text { Date } \\
\text { Measured } \\
\end{array}$} \\
\hline & & & & Size & Interval & & & & & \\
\hline Hagestad-1 & $\begin{array}{l}\text { N889,190 } \\
\mathrm{E} 631,132\end{array}$ & 1957 & 1941 & $51 / 2 "$ & $0-1941$ & $\begin{array}{c}675-685 \\
990-1000 \\
1600-1620 \\
1750-1770 \\
1885-1895 \\
1905-1915\end{array}$ & $\begin{array}{l}\text { Jirtiary } \\
\text { Volcanics } \\
\text { Undivided }\end{array}$ & 7485.3 & $<5912.9$ & 1963 \\
\hline $\begin{array}{l}\text { Test } \\
\text { Well } 1\end{array}$ & $\begin{array}{l}\text { N876,855 } \\
\text { E629,310 }\end{array}$ & 1961 & 4198 & \begin{tabular}{cc|}
11 & $3 / 4 "$ \\
8 & $5 / 8 "$
\end{tabular} & $\begin{array}{c}0-1615 \\
1563-3700\end{array}$ & $\begin{array}{l}1910-1950 \\
2030-2050 \\
2100-2160 \\
2230-2270 \\
2370-2430\end{array}$ & $\begin{array}{l}\text { Jertiary } \\
\text { Volcanics } \\
\text { Undivided } \\
\text { Devonian } \\
\text { Dolomite }\end{array}$ & 6156 & $\begin{array}{l}4715 \\
4691 \\
4691 \\
4189\end{array}$ & $\begin{array}{c}1963 \\
1987^{+} \\
1991^{*} \\
1963 \text { for } \\
\text { Dolomite } \\
\end{array}$ \\
\hline U12e.03-1 & $\begin{array}{l}\text { N888,264 } \\
\text { E634,169 }\end{array}$ & 1959 & 855 & $\begin{array}{l}\text { 6" } \\
4 " \\
\text { 3" }\end{array}$ & $\begin{array}{c}0-5 \\
0-509 \\
0-702\end{array}$ & None & $\begin{array}{c}\text { Tertiary } \\
\text { Volcanics } \\
\text { Undivided } \\
\text { Devonian } \\
\text { Dolomites } \\
\end{array}$ & 6150 & $<5440$ & 1959 \\
\hline U12e.M1 & $\begin{array}{l}\text { N886,644 } \\
\text { E633,532 }\end{array}$ & 1960 & $\begin{array}{c}1501 \\
\text { (spud } \\
\text { within } \\
\text { U12e } \\
\text { tunnel) }\end{array}$ & $33 / 8 "$ & $0-854$ & None & $\begin{array}{c}\text { Tirtiary } \\
\text { Volcanics } \\
\text { Undivided } \\
\text { Devonian } \\
\text { Dolomites } \\
\end{array}$ & 6158 & $<4673$ & 1960 \\
\hline $\begin{array}{l}\text { Dolomite } \\
\text { Hill Hole }\end{array}$ & $\begin{array}{l}\text { N886,712 } \\
\text { E638,632 }\end{array}$ & 1959 & 1200 & $6^{\prime \prime}$ & $0-40$ & None & $\begin{array}{l}\text { Devonian } \\
\text { Dolomites }\end{array}$ & 6399 & $<5375$ & 1960 \\
\hline U12e.06-1 & $\begin{array}{l}\text { N885,038 } \\
\mathrm{E} 631,776\end{array}$ & 1962 & 3180 & $27 / 8 "$ & $0-3179$ & $0-3114$ & $\begin{array}{c}\text { Tertiary } \\
\text { Volcanics } \\
\text { Undivided } \\
\text { Devonian } \\
\text { Dolomites }\end{array}$ & 7573 & $<4643$ & 1962 \\
\hline
\end{tabular}




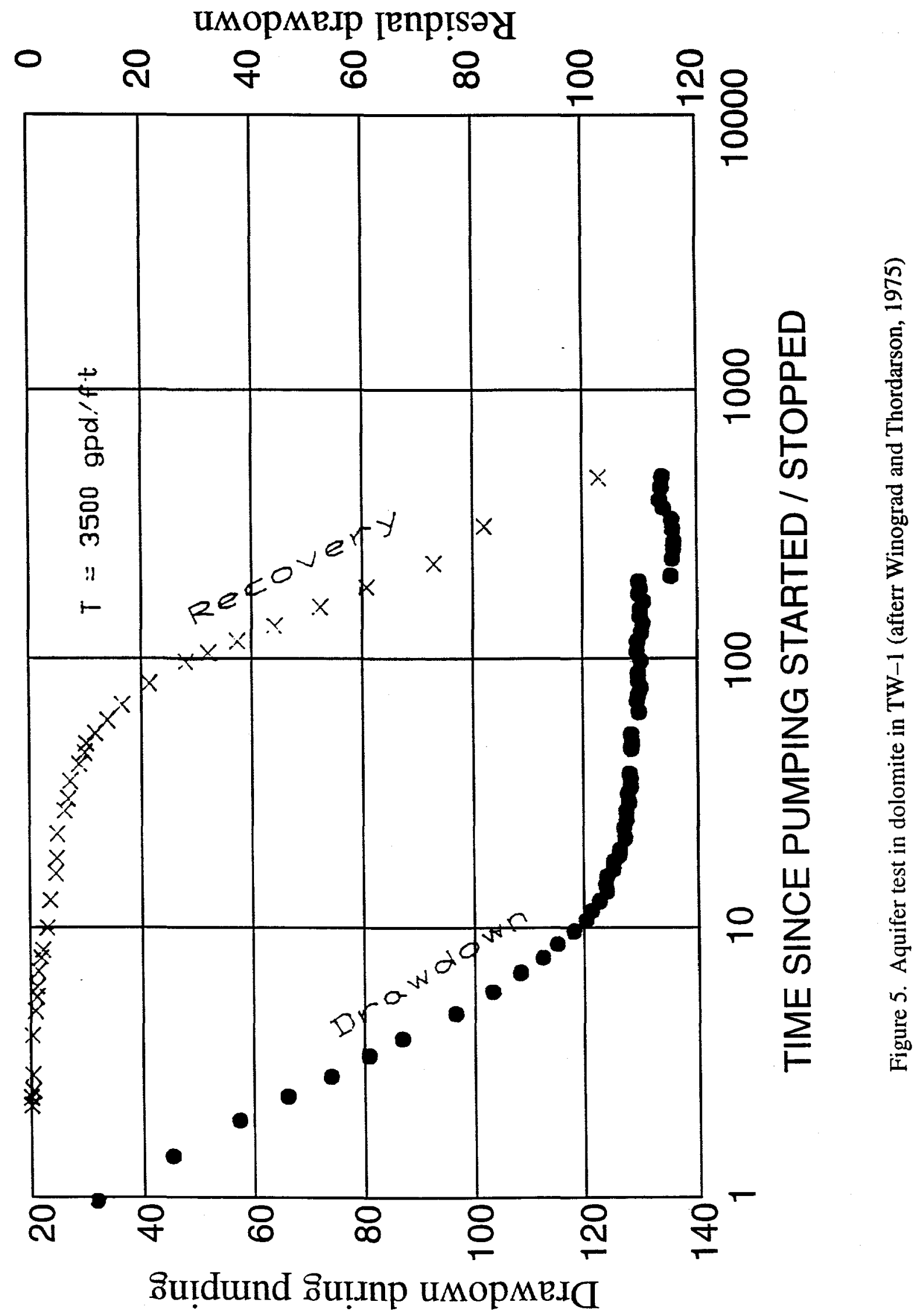


bailing tests of the drilling fluid stored within the Simonson Dolomite in the Dolomite Hill Hole indicated that fluid levels recovered very slowly $(0.5 \mathrm{ft} / \mathrm{min}$ to $1 \mathrm{ft} /$ hour $)$. The relatively slow recovery rates are another qualitative indication of relatively low transmissivities for the Simonson Dolomite.

A series of slug tests were performed on a 646-foot section of open drillhole in U12e.M1. One hundred twenty $\mathrm{ft}$ of the open hole consisted of Tertiary tuffs and $527 \mathrm{ft}$ consisted of the Guilmette Formation and/or the Simonson Dolomite undivided. The results indicated a transmissivity of 1 to $2 \mathrm{gal} / \mathrm{day} / \mathrm{ft}$. These values were calculated from a slug test conducted in an undeveloped well and marred by inaccurate injection volumes. The values obtained were deemed suspect by Schoff and Winograd (1961) owing to the unknown effect of bentonite mud previously lost in the borehole. and should be used only in a qualitative sense.

Winograd and Thordarson (1975) report transmissivities measured within the Lower Carbonate Aquifer at different areas around the Nevada Test Site (which includes the carbonate units that may be encountered at the ER-12-1 drill site) between 1300 to 86,000 $\mathrm{gal} /$ day/ft. If the data gathered from Test Well 1. Dolomite Hill Hole and U12e.M1 are representative of conditions at the ER-12-1 drill site, then the transmissivities measured within ER-12-1 should fall toward the lower end of these values.

Examinations of cores from U12e.06-1, U12b.07-1, and Dolomite Hill Hole indicate that the effective porosity ranges from 0.6 to 1.1 percent and the interstitial permeability ranges from 0.0002 to $0.00007 \mathrm{gal} / \mathrm{day} / \mathrm{sq}$. $\mathrm{ft}$ (Thordarson, 1965). These data, in conjunction with the aquifer-test data, suggest that secondary porosity is the primary fluid transport pathway within the Devonian dolomites. If true, the transmissivities of the units are controlled by the frequency, aperature, and continuity of fractures and faults.

The Eleana Formation has been hydraulically tested south of the ER-12-1 drill site using wells UE-16d (N844 878 E646 567) and UE-16f (N832 355 E648 843). Aquifer tests conducted within unit $J$ of the Eleana Formation yielded transmissivities of 8 and 370 $\mathrm{gal} / \mathrm{day} / \mathrm{ft}$ (Figures 6 and 7). The ER-12-1 drillhole may encounter units $\mathrm{D}$ and lower of the Eleana Formation. Transmissivities in these units may be similar to those measured in unit $\mathrm{J}$ at UE-16d and UE-16f. If this is correct. the permeability of the Eleana Formation (as measured at the ER-12-1) may be an order of magnitude less than that of the Devonian dolomites. The large distance ( 8 miles) between the drill site and wells UE-16d and UE-16f, and the different structural and depositional histories of the two sites. makes this interpolation tentative.

Vertical Gradients

Large vertical gradients exist between the Tertiary volcanics and the Devonian dolomite units at Test Well 1 (Table 2). indicating vertical flow from the tuffs to the dolomites. A recent flow survey conducted by the USGS and DRI on Test Well 1 within the Tertiary volcanics 


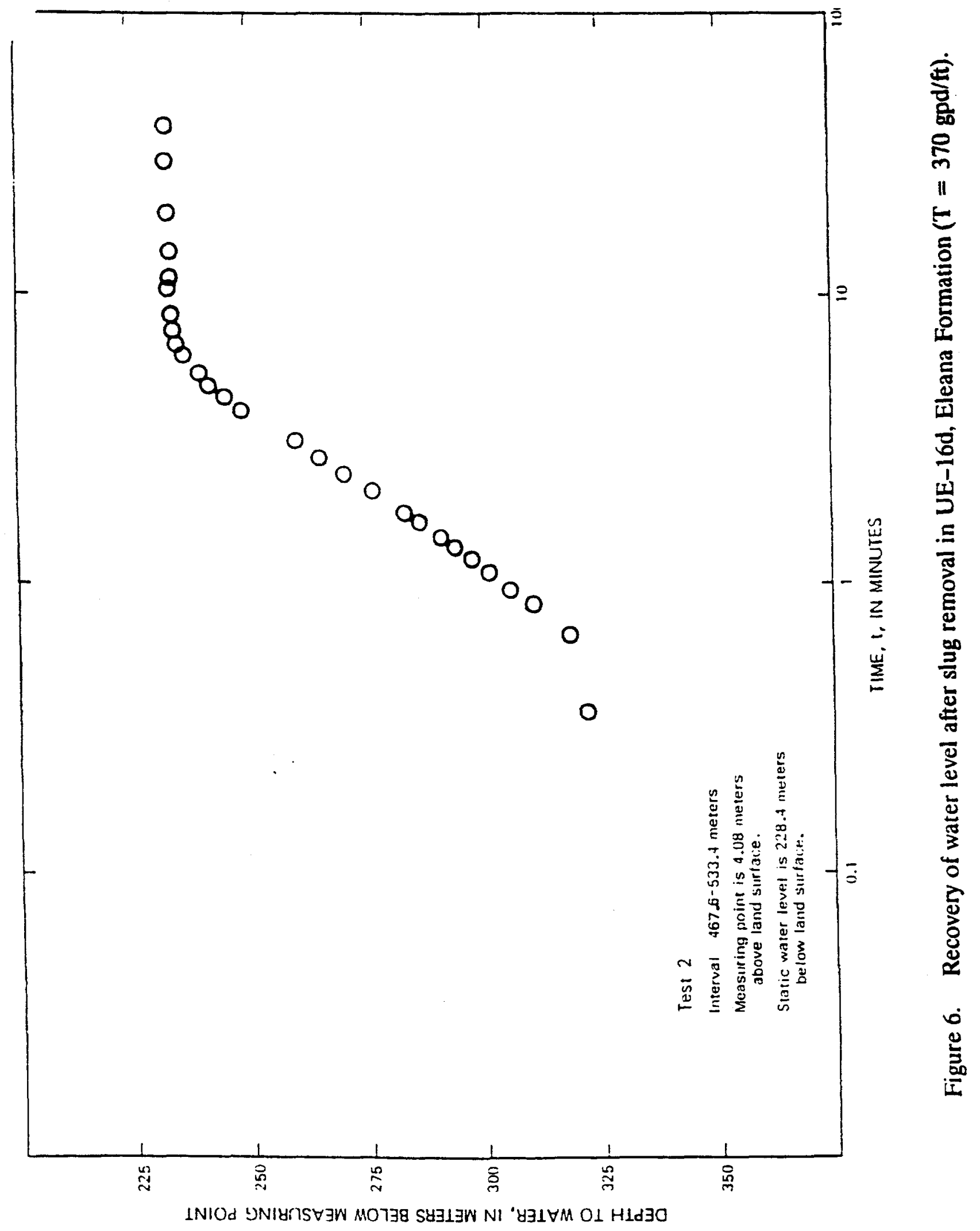




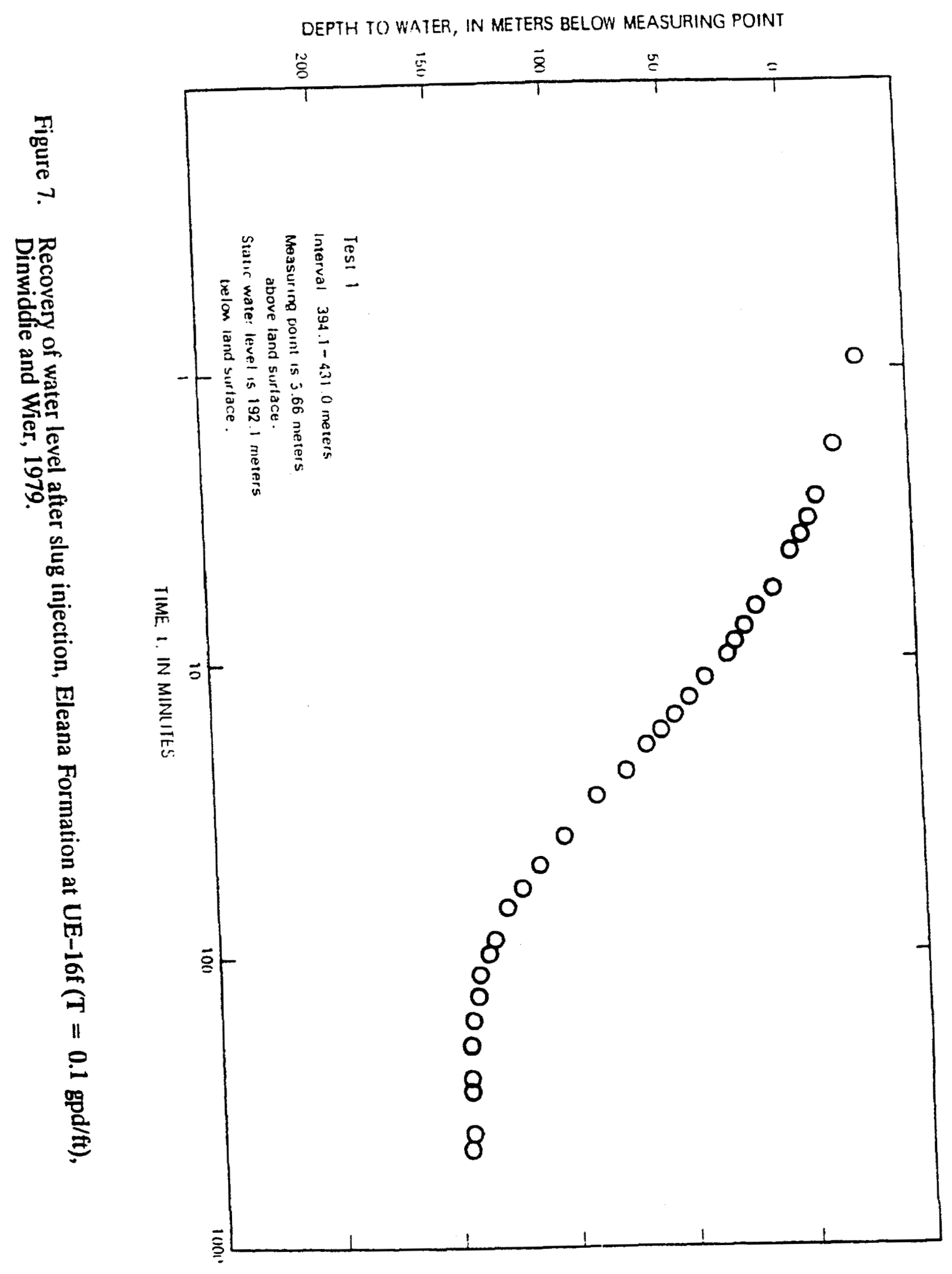


indicated upward flow occurring between 4691 and $4206 \mathrm{ft}$ elevation and downward flow between 4126 and $3726 \mathrm{ft}$. Vertical flow may occur in the dolomites as well. Rainier Mesa is a recharge area (Clebsch, 1961; Thordarson, 1965; and Russell, 1988), and as such, decreasing heads with depth may exist within the carbonates for most of the area, including the ER-12-1 drill site, indicating the presence of vertical flow.

Vertical gradients have not been measured within the Eleana Formation in the vicinity of Rainier Mesa. Hydraulic-head measurements from wells UE-16d and UE-16f increase with depth (Dinwiddie and Wier, 1979). This phenomenon may be related to the Eleana Formation acting as a groundwater-flow barrier between the Alkali Flat/Furnace Creek subbasin to the west and the Ash Meadows subbasin to the east. Gradients for upward flow may or may not exist in the Eleana Formation at the ER-12-1 drill site, depending upon local hydrodynamics.

Geochemistry

Geochemical samples were collected on two occasions from Test Well 1. These data are presented in Table 3. Samples were collected $70 \mathrm{ft}$ below the volcanic/dolomite contact and are typical of samples taken from tuffaceous rocks $\left(\mathrm{Na}-\mathrm{HCO}_{3}\right.$ water type). The geochemistry and sample environment suggest that the groundwater within the dolomites originates from or mixes with groundwater from the overlying tuffaceous sediments. Low tritium values indicate that the water within Test Well 1 recharged prior to atmospheric testing which was conducted during the 1950s and early 1960s. Groundwater at the ER-12-1 drill site will probably be a $\mathrm{Na}-\mathrm{Ca}-\mathrm{HCO}_{3}$ water type which is characteristic of water that has reacted or mixed with water from both the tuffs and carbonate rocks. Elevated levels of tritium may be found within groundwater at the ER-12-1 drill site because of the drill site's proximity to the U12e tunnel effluent ponds. A slight potential exists for groundwater to exceed national primary drinking water regulations, maximum contaminant levels (40 CFR, Chapter 1, Part 141 , subpart B) for tritium ( $>20,000 \mathrm{pCi} / \mathrm{L})$.

\begin{tabular}{|c|c|c|c|c|c|c|c|c|c|c}
\hline \multicolumn{10}{|c}{ TABLE 3. AQUEOUS GEOCHEMISTRY FOR TEST WELL 1. } \\
\hline $\begin{array}{c}\text { Sample } \\
\text { Date }\end{array}$ & $\begin{array}{c}\mathbf{3 H}^{+} \\
\mathbf{p C i} / \mathbf{m l}\end{array}$ & $\begin{array}{c}\mathbf{E C} \\
(\mathbf{S})\end{array}$ & $\mathbf{p H}$ & $\begin{array}{c}\mathrm{Ca} \\
(\mathbf{m g} / \mathrm{L})\end{array}$ & $\begin{array}{c}\mathbf{N a} \\
(\mathbf{m g} / \mathbf{L})\end{array}$ & $\begin{array}{c}\mathbf{S i O}_{\mathbf{2}} \\
(\mathbf{m g} / \mathbf{L})\end{array}$ & $\begin{array}{c}\mathbf{H C O}_{3} \\
(\mathbf{m g} / \mathbf{L})\end{array}$ & $\begin{array}{c}\mathbf{C O}_{3} \\
(\mathbf{m g} / \mathbf{L})\end{array}$ & $\begin{array}{c}\mathrm{Cl} \\
(\mathbf{m g} / \mathbf{L})\end{array}$ & $\begin{array}{c}\mathbf{S O}_{4} \\
(\mathbf{m g} / \mathbf{L})\end{array}$ \\
\hline $7 / 28 / 87$ & 15 & 220 & 8 & 2 & 48 & 3 & 117 & 6 & 3 & 1 \\
\hline $9 / 26 / 90$ & 10 & 225 & 9 & 2 & 51 & 2 & 93 & 18 & 3 & - \\
\hline
\end{tabular}

\section{SUMMARY}

The ER-12-1 drill site is potentially downgradient from Rainier Mesa where underground nuclear tests have been conducted and tunnel effluent ponds have been in use. Saturated hydrologic units expected to be encountered at this drill site inciude the Simonson Dolomite, the "cherty-argillaceous unit," the Sevy Dolomite, and the Eleana Formation. 
These units correspond to Winograd and Thordarson's (1975) lower carbonate aquifer and the upper clastic aquitard. The water table should be encountered within the Simonson Dolomite at an approximate altitude of $4200 \pm 100 \mathrm{ft}$. Decreasing hydraulic heads with depth may be encountered. The dolomites encountered within ER-12-1 should exhibit a wide range of transmissivities depending upon the degree and continuity of fractures and faults. However, the transmissivities should be similar to those measured in Test Well 1 (3500 $\mathrm{gal} / \mathrm{day} / \mathrm{ft}$ ). Transmissivities within the Eleana Formation are expected to be less than that in the dolomites by at least an order of magnitude. Aqueous geochemistry samples should exhibit an $\mathrm{Na}-\mathrm{Ca}-\mathrm{HCO}_{3}$ water type, which is characteristic of groundwater that has reacted or mixed with tuffaceous and carbonate rocks.

\section{REFERENCES}

Clebsch. A., 1961. Tritium age of groundwater at the NTS, Nye County Nevada: U.S. Geological Survey Professional Paper 424-C, p. C122-125.

Dinwiddie, G.A. and J.E. Wier, 1979. Summary of hydraulic tests and hydraulic data for holes UE-16d and UE-16f, Syncline Ridge Area. Nevada Test Site: U.S. Geological Survey Report USGS-1543-3.20 p.

Gibbons, A.B., E.N. Hinrichs, W.R. Hansen and R.N. Lemke, 1963. Geology of the Rainier Mesa Quadrangle, Nye County Nevada: U.S. Geological Survey Geologic Quadrangle Map GQ-215.

Russell, C.E., 1988. Hydrogeologic investigation of flow in fractured tuff, Rainier Mesa, Nevada Test Site: Desert Research Institute Report 45062, Las Vegas and Reno, Nevada, $47 \mathrm{p}$.

Schoff, S.L. and I.J. Winograd, 1961. Hydrologic significance of six coreholes in carbonate rocks of the Nevada Test Site: U.S. Geological Survey Open-File Report TEI-787, 97 p.

Thordarson, W., 1965. Perched groundwater in zeolitized-bedded tuff, Rainier Mesa and vicinity, Nevada Test Site. Nevada: U.S. Geological Survey Open-File Report TEI-862, $85 \mathrm{p}$.

Thordarson, W., R.A. Young and I.J. Winograd. 1967. Records of wells and test holes in the Nevada Test Site and vicinity (through December, 1966): U.S. Geological Survey Open-File Report TEI-872, 23 p.

Waddell, R.K.. J.H. Robison and R.K. Blankennagel, 1984. Hydrology of Yucca Mountain and vicinity, Nevada-California-investigative results through mid-1983:U.S. Geological Survey Water-Resources Investigations Report 84-4267, 40 p.

Winograd, I.J. and W. Thordarson, 1975. Hydrogeologic and hydrochemical framework, south-central Great Basin, Nevada-California, with special reference to the Nevada Test Site: U.S. Geological Survey Professional Paper 712-C, 126 p. 


\section{APPENDIX C}

\section{ABRIDGED DRILL HOLE STATISTICS FOR ER-12-1}

\section{Location}

Coordinates: $\quad$ N270,248.5 E195,236.6 $\mathrm{m} \quad \mathrm{N} 886,640.26 \quad$ E640,538.85 ft

Ground Elevation: $1773.51 \mathrm{~m} \quad 5817.12 \mathrm{ft}$

Top of $13.97 \mathrm{~cm}(5.5 \mathrm{in})$ casing is at an elevation of $1774.0 \mathrm{~m}(5818.19 \mathrm{ft})$

\section{Drilling data}

Spud Date: 19 July 91

Drill Rig: Cardwell 500

Total Depth (TD): $1093.6 \mathrm{~m} \quad 3588 \mathrm{ft}$

Date Reached TD: 17 October 91

Summary of Drilling Technique:

Rotary drilled surface hole to $16.2 \mathrm{~m}(53 \mathrm{ft}$ ) using mud and convention circulation. Rotary drilled $0.47 \mathrm{~m}$ ( $18.5 \mathrm{in}$ ) hole to $30.5 \mathrm{~m}$ (100 ft) using water and conventional circulation, used reverse circulation with air and water to $65.5 \mathrm{~m}(215 \mathrm{ft})$. Drilled $0.445 \mathrm{~m}(17.5 \mathrm{in})$ hole to $520.9 \mathrm{~m}(1709 \mathrm{ft})$ using an Air Hammer with conventional circulation and air/foam. Rotary drilled $0.311 \mathrm{~m}$ (12.25 in) hole using conventional circulation and airfoam from 520.9 to $556.9 \mathrm{~m}$ (1709 to $1827 \mathrm{ft}$ ). Continued $0.311 \mathrm{~m}(12.25 \mathrm{in})$ hole using conventional circulation and bentonite mud from $556.9 \mathrm{~m}$ (1827 $\mathrm{ft}$ ) to TD.

Cut four conventional cores:

$\# 1$ at 539.2 to $543.8 \mathrm{~m}(1769$ to $1784 \mathrm{ft})$

$\# 2$ at 548.0 to $556.3 \mathrm{~m}(1798$ to $1825 \mathrm{ft})$

\#3 at 751.0 to $757.7 \mathrm{~m}(2464$ to $2486 \mathrm{ft})$

\#4 at 895.2 to $903.1 \mathrm{~m}$ ( 2937 to $2963 \mathrm{ft}$ )

\section{Casing record}

Surface to $15.8 \mathrm{~m}(52 \mathrm{ft})$

Outside diameter: $0.508 \mathrm{~m}$ (20 in)

Weight: $104.13 \mathrm{lb} / \mathrm{ft}$ 
Intermediate to $449.3 \mathrm{~m}(1474 \mathrm{ft})^{*}$

Outside diameter: $0.34 \mathrm{~m}(13.375 \mathrm{in})$

Weight: $54.5 \mathrm{lb} / \mathrm{ft}$

*Although $450.6 \mathrm{~m}$ (1478.27 ft) was reported by the Driller, geophysical logs indicate bottom of casing at $449.3 \mathrm{~m}(1474 \mathrm{ft})$.

Completion string to $1072.9 \mathrm{~m}(3520 \mathrm{ft})$

Outside diameter: $0.219 \mathrm{~m}(8.625 \mathrm{in})$

Type: Carbon Steel

Screened intervals:

1. 516.0 to $555.0 \mathrm{~m}$ (1693 to $1821 \mathrm{ft}$ )

2. 585.2 to $597.4 \mathrm{~m}$ (1920 to $1960 \mathrm{ft}$ )

3. 764.7 to $790.6 \mathrm{~m}$ ( 2509 to $2594 \mathrm{ft}$ )

4. 911.3 to $963.8 \mathrm{~m}$ (2990 to $3162 \mathrm{ft}$ )

5. 1023.5 to $1049.1 \mathrm{~m}$ ( 3358 to $3442 \mathrm{ft}$ )

Gravel packed intervals:

6. 500.2 to $562.7 \mathrm{~m}$ (1641 to $1846 \mathrm{ft}$ )

7. 573.9 to $591.3 \mathrm{~m}$ (1883 to $1940 \mathrm{ft}$ )

8. 746.4 to $793.1 \mathrm{~m}$ ( 2449 to $2602 \mathrm{ft}$ )

9. 901.6 to $979.0 \mathrm{~m}$ ( 2958 to $3212 \mathrm{ft}$ )

10. 1008.6 to $1040.6 \mathrm{~m}$ (3309 to $3414 \mathrm{ft}$ )

Production string to $1047.0 \mathrm{~m}(3435 \mathrm{ft})$

Outside diameter: $0.14 \mathrm{~m}$ (5.5 in)

Type: Carbon Steel

Location of packers:

11. 510.6 to $512.8 \mathrm{~m}$ ( 1675 to $1682 \mathrm{ft}$ )

12. 557.3 to $559.5 \mathrm{~m}$ (1828 to $1836 \mathrm{ft}$ )

13. 582.4 to $584.5 \mathrm{~m}$ (1911 to $1918 \mathrm{ft}$ ) 
14. 599.3 to $601.5 \mathrm{~m}$ (1966 to $1973 \mathrm{ft}$ )

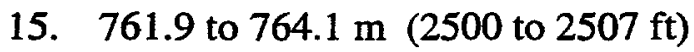

16. 793.4 to $795.6 \mathrm{~m} \mathrm{(2603} \mathrm{to} 2610 \mathrm{ft}$ )

17. 907.9 to $910.1 \mathrm{~m} \mathrm{(2979} \mathrm{to} 2986 \mathrm{ft}$ )

18. 965.2 to $967.4 \mathrm{~m}$ ( 3167 to $3174 \mathrm{ft}$ )

19. 1020.5 to $1022.7 \mathrm{~m}$ ( 3348 to $3355 \mathrm{ft}$ )

Location of sliding sleeves:

20. 536.3 to $536.8 \mathrm{~m}(1759$ to $1761 \mathrm{ft})$

21. 590.6 to $591.8 \mathrm{~m}$ (1938 to $1942 \mathrm{ft}$ )

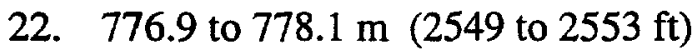

23. 938.6 to $939.8 \mathrm{~m} \mathrm{(3079} \mathrm{to} 3083 \mathrm{ft}$ )

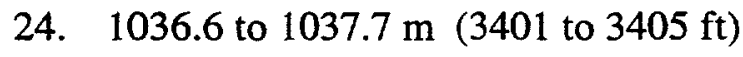

\section{Well site geology}

Raytheon Services Nevada

\section{Drilling Contractor:}

Reynolds Electrical and Engineering Company

\section{Geophysical logs:}

Atlas Wireline Services (main logging suite), Schlumberger, Lawrence Livermore National Laboratory, Desert Research Institute (specialty logs), Westech Engineering (downhole camera).

\section{Hydrologic testing:}

Desert Research Institute (pumping tests) and Schlumberger (straddle packer tests) 


\section{APPENDIX D \\ SEQUENCE OF EVENTS AT ER-12-1}

19 Jul 91

19 Aug 91

20 Aug 91 through 21 Aug 91

22 Aug 91 through 3 Sept 91

4 Sep 91

17 Oct 91

18 Oct 91 through 22 Oct 91

23 Oct 91

28 Oct 91

13 Jan 92 through 24 Jan 92

25 Jan 92 through 31 Jan 92

20 Feb 92 through 24 Feb 92

25 Feb 92

26 Feb 92 through 28 Feb 92

05 Mar 92 through 06 Mar 92

07 Mar 92 through 11 Mar 92

12 Mar 92 through 15 Mar 92

16 Mar 92 through 6 Apr 92

14 Sep 92 through 21 Sep 92

24 Sep 92 through 2 Oct 92

16 Oct 92 through 23 Oct 92

5 Jan 93 through 6 Jan 93 begin drilling

penetrate static water level at aproximately $469.4 \mathrm{~m}$ $(1540 \mathrm{ft})$

conduct dry hole geophysical logs

set intermediate casing to $657.1 \mathrm{~m}(2156 \mathrm{ft})$

collect water sample from $509.3 \mathrm{~m}(1671 \mathrm{ft})$

total depth reached at $1093.6 \mathrm{~m} \mathrm{(3588 \textrm {ft } )}$

conduct wet hole geophysical logs

Begin initial well development using sodium tetraphosphate

stop well development due to hole instability

clean and condition hole using first mud and then polymer

set $19.4 \mathrm{~cm}$ (7 5/8 in) intermediate casing to TD

continue well development using sodium tetraphosphate to clean hole

remove fluid from well by "dipstick" method

remove fluid from well by "swabbing" method

pump well using medium-size pump

replace medium-size pump with small-size pump

develop well by pumping with small-size pump

conduct aquifer tests and initial chemistry samples

develop well using Sodium Acid Pyrophosphate and air-lifting fluids from well

conduct straddle packer tests

set $14 \mathrm{~cm}(51 / 2$ in) casing with packers and sliding sleeves in well

conduct aquifer test and geochemical sampling (upper zone only) 


\section{DISTRIBUTION}

Bob Bangerter

Environmental Restoration Division

Nevada Operations Office

U.S. Department of Energy

P.O. Box 98518

Las Vegas, NV 89193-8518

Joanne M. Bradbery, Director

Contract Management Division

Nevada Operations Office

U.S. Department of Energy

P.O. Box 98518

Las Vegas, NV 89193-8518

David Bedsun

Defense Special Weapons Agency

Field Command

Nevada Operations Office

P.O. Box 208

Mercury, NV 89023-0208

Mary Lou Brown

International Technology Corporation 4330 S. Valley View

Suite 114

Las Vegas, NV 89103

James Cebe

Energy Technologies Division

Nevada Operations Office

U.S. Department of Energy

P.O. Box 98518

Las Vegas, NV 89193-8518

Beverly Colbert

Contract Management Division

Nevada Operations Office

U.S. Department of Energy

P.O. Box 98518

Las Vegas, NV 89193-8518

Frank Di Sanza, Director

Energy Technologies Division

Nevada Operations Office

U.S. Department of Energy

P.O. Box 98518

Las Vegas, NV 89193-8518
Brian Dozier

Bechtel Nevada Corporation

P.O. Box 98521

Las Vegas, NV 89193-8521

Doug Duncan

Hydrology Program Manager

Environmental Protection Division

Nevada Operations Office

U.S. Department of Energy

P.O. Box 98518

Las Vegas, NV 89193-8518

Dennis Farmer

Radiation Sciences Laboratory

Office of Radiation and Indoor Air

U.S. Environmental Protection Agency

P.O. Box 98517 , M/S 513

Las Vegas, NV 89193-8517

Larry Franks

Nevada State Health Department

Radiological Health Section

620 Belrose Avenue

Las Vegas, NV 89158

Joseph M. Ginanni

Waste Management Division

Nevada Operations Office

U.S. Department of Energy

P.O. Box 98518

Las Vegas, NV 89193-8518

Kenneth Hoar, Director

Environmental Protection Division

Nevada Operations Office

U.S. Department of Energy

P.O. Box 98518

Las Vegas, NV 89193-8518

Kathy Izell

Assistant Manager for Technical Services

Nevada Operations Office

U.S. Department of Energy

P.O. Box 98518

Las Vegas, NV 89193-8518 
Roger Jacobson

Desert Research Institute

Water Resources Center

P.O. Box 19040

Las Vegas, NV 89132-0040

\section{Marjory Jones}

Desert Research Institute

Water Resources Center

P.O. Box 60220

Reno, NV 89506-0220

Jim Kannard

Bechtel Nevada Corporation

P.O. Box 98521

Las Vegas, NV 89193-8521

Randy Laczniak

U.S. Geological Survey

Water Resources Division

6770 S. Paradise Rd.

Las Vegas, NV 89119

Steve Lawrence

Engineering Division

Nevada Operations Office

U.S. Department of Energy

P.O. Box 98518

Las Vegas, NV 89193-8518

Steve Leedom

Stockpile Stewardship Division

Nevada Operations Office

U.S. Department of Energy

P.O. Box 98518

Las Vegas, NV 89193-8518

Charles E. McWilliam, Director

Defense Projects Division

Nevada Operations Office

U.S. Department of Energy

P.O. Box 98518

Las Vegas, NV 89193-8518

Steve Mellington, Director

Environmental Restoration Division

Nevada Operations Office

U.S. Department of Energy

P.O. Box 98518

Las Vegas, NV 89193-8518
Leslie A. Monroe

Environmental Protection Division

Nevada Operations Office

U.S. Department of Energy

P.O. Box 98518

Las Vegas, NV 89193-8518

Ken Rehfeldt

Geotrans, clo IT

4330 Valley View

Suite 112, MS-439

Las Vegas, NV 89103

Stuart E. Rawlinson

Bechtel Nevada Corporation

P.O. Box 95487 , M/S 580

Las Vegas, NV 89193-5487

Monica Salazar-Sanchez

Environmental Restoration Division

Nevada Operations Office

U.S. Department of Energy

P.O. Box 98518

Las Vegas, NV 89193-8518

Ralph Smiecinski

Energy Technologies Division

Nevada Operations Office

U.S. Department of Energy

P.O. Box 98518

Las Vegas, NV 89193-8518

David K. Smith

Isotopes Sciences Division

Lawrence Livermore National Laboratory

P.O. Box 808, M/S L231

Livermore, CA 94550

Michael J. Sully

Bechtel Nevada Corporation

P.O. Box 98521 , M/S 966

Las Vegas, NV 98193-8521

Joe Thompson

Los Alamos National Laboratory

INC-11, MS J514

P.O. Box 1663

Los Alamos, NM 87545 
Doug Trudeau

U.S. Geological Survey

Water Resources Division

6770 S. Paradise Rd.

Las Vegas, NV 89119

Janet Wiley

International Technology Corporation 4330 S. Valley View

Suite 114

Las Vegas, NV 89103

Annie Kelley

State Documents Department

Nevada State Library

Capitol Complex

Carson City, NV 89710

Archives

Getchell Library

University of Nevada, Reno

Beverly Carter

MacKay School of Mines Library

University of Nevada, Reno

Document Section, Library

University of Nevada, Las Vegas

4505 Maryland Parkway

Las Vegas, NV 89154

Library (Stead)

Desert Research Institute

P.O. Box 60220

Reno, Nevada 89506-0220
Library

IT Corporation

4330 S. Valley View

Suite 114

Las Vegas, NV 89103

ATTN: Toni Miller

Library

Southem Nevada Science Center

Desert Research Institute

P.O. Box 19040

Las Vegas, NV 89132-0040

Public Reading Facility

Bechtel Nevada Corporation

P.O. Box 98521

Las Vegas, NV 89193-8521

Technical Information Resource Center

Nevada Operations Office

U.S. Department of Energy

P.O. Box 98518

Las Vegas, NV 89193-8518

Librarian

Water Resources Center Archives

410 O'Brien Hall

University of California

Berkeley, CA 94720-1718

Office of Scientific and Technical Information

U.S. Department of Energy

P.O. Box 62

Oak Ridge, TN 37831-9939 
